\title{
FINAL REPORT ON GRAPHITE IRRADIATION TEST OG-2
}

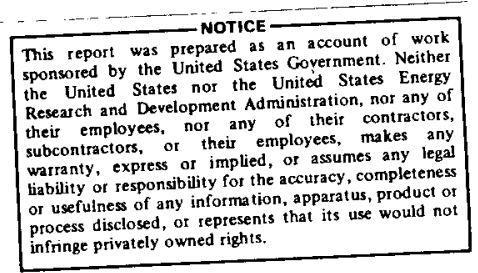

Thic nonment is

PUBL Tavys gathem Authorizing Official$$
\text { D }
$$

\author{
by \\ R. J. PRICE and L. A. BEAVAN
}




\section{DISCLAIMER}

This report was prepared as an account of work sponsored by an agency of the United States Government. Neither the United States Government nor any agency Thereof, nor any of their employees, makes any warranty, express or implied, or assumes any legal liability or responsibility for the accuracy, completeness, or usefulness of any information, apparatus, product, or process disclosed, or represents that its use would not infringe privately owned rights. Reference herein to any specific commercial product, process, or service by trade name, trademark, manufacturer, or otherwise does not necessarily constitute or imply its endorsement, recommendation, or favoring by the United States Government or any agency thereof. The views and opinions of authors expressed herein do not necessarily state or reflect those of the United States Government or any agency thereof. 


\section{DISCLAIMER}

Portions of this document may be illegible in electronic image products. Images are produced from the best available original document. 
ABSTRACT

This report describes the results of dimensional, thermal expansivity, thermal conductivity, Young's modulus, and tensile strength measurements on specimens of nuclear graphites irradiated in capsule OG-2. About half the irradiation space was allocated to $\mathrm{H}-451$ near-isotropic petroleum-cokebased graphite or its subsized prototype grade H-429. Most of these specimens had been previously irradiated. Virgin specimens of another nearisotropic graphite, grade TS-1240, were irradiated. Some previously irradiated specimens of needle-coke-based H-327 graphite and pitch-cokebased $\mathrm{P}_{3}$ JHAN were also included.

Data were obtained in the irradiation temperature range $600^{\circ}$ to $1400^{\circ} \mathrm{C}$. Accumulated neutron fluences now exceed $8 \times 10^{21} \mathrm{n} / \mathrm{cm}^{2}$ (E $\left.>0.18 \mathrm{MeV}\right)$ on $\mathrm{H}-429$ graphite and $6 \times 10^{21} \mathrm{n} / \mathrm{cm}^{2}(\mathrm{E}>0.18 \mathrm{MeV})$ on $\mathrm{H}-451, \mathrm{P}_{3} \mathrm{JHAN}$, and H-327 graphites.

The near-isotropic graphites continued to show the good dimensional stability indicated in previous irradiation experiments. Maximum observed shrinkages in $\mathrm{H}-451 / \mathrm{H}-429$ were $2 \%$ in the radial direction and $3.5 \%$ in the axial direction, and no net expansion was observed at the irradiation conditions investigated. The low fluence data on TS-1240 graphite indicated dimensional stability as good as, or better than, that of H-451 graphite.

Irradiation-induced changes in the thermal properties followed previously established patterns. The thermal expansivity of the nearisotropic graphites increased marginally at low irradiation temperatures and decreased by up to $30 \%$ at high irradiation temperatures. Irradiation reduced the thermal conductivity, with the largest reductions taking place at the lowest temperatures. 
Both Young's modulus and tensile strength increased significantly after irradiation for all the tested graphites. The relation between irradiationinduced changes in Young's modulus, E, and strength, S, predicted by the constant strain energy failure criterion,

$$
\frac{S_{i}}{S_{0}}=\sqrt{\frac{E_{i}}{E_{0}}},
$$

was approximately obeyed, but the tensile strength of irradiated $\mathrm{H}-451$ specimens and many of the H-327 specimens was somewhat higher than predicted. 
CONTENTS

ABSTRACT . . . . . . . . . . . . . . . . . . . 1 . . . . . . .

1. INTRODUCTION . . . . . . . . . . . . . . . . . . . . 1-1

1.1. Scope of Report . . . . . . . . . . . . . . 1-1

1.2. Fast Fluence Units . . . . . . . . . . . . . . 1-1

2. TEST DESCRIPTION AND OBJECTIVES . . . . . . . . . . . . . 2-1

3. MATERIAlS INVESTIGATED . . . . . . . . . . . . . . . 3-1

4. CAPSUlE OPERATING CONDITIONS . . . . . . . . . . . . . . 4-1

4.1. Description of Capsule . . . . . . . . . . . 4-1

4.2. Operating Temperatures ... . . . . . . . . . 4-3

4.3. Neutron Fluence . . . . . . . . . . . . . . 4-5

5. EXPERIMENTAL RESULTS - DIMENSIONAL CHANGES . . . . . . . . 5-1

5.1. Irradiation-Induced Dimensional Change in Near-Isotropic Graphites . . . . . . . . . . . . . . . 5-1

5.2. Dimensional Change in Needle Coke Graphites . . . . . 5-43

5.3. Miscellaneous Graphites ............. . 5-55

6. EXPERIMENTAL RESULTS - THERMAL PROPERTIES . . . . . . . . . 6-1

6.1. Thermal Expansivity ............... 6-1

6.2. Thermal Conductivity . . . . . . . . . . 6-8

7. EXPERIMENTAL RESULTS: TENSILE PROPERTIES . . . . . . . . . 7-1

8. IMPACT OF TEST DATA ON CURRENT DESIGN PROCEDURES . . . . . . . 8-1

8.1. Dimensional Changes . . . . . . . . . . . . 8-1

8.2. Thermal Expansivity . . . . . . . . . . . 8-1

8.3. Thermal Conductivity . . . . . . . . . . . 8-1

8.4. Mechanical Properties . . . . . . . . . . . 8-2

9. REFERENCES ......................... . 9-1

FIGURES

4-1. Schematic layout of capsule OG-2 with thermocouple locations . . . . . . . . . . . . . . . . 4-2

4-2. Measured fast $(\mathrm{E}>0.18 \mathrm{MeV})_{\mathrm{ORR}}$ and thermal fluence values
for the $\mathrm{OG}-2$ experiment . . . . . . . . . . . . $7-7$ 


\section{FIGURES (continued)}

5-1(a). Dimensional change of $\mathrm{H}-451 / \mathrm{H}-429$ graphite, axial

orientation, as a function of fast neutron fluence at

irradiation temperatures of $550^{\circ}$ and $750^{\circ} \mathrm{C}$. . . . . . 5-32

5-1(b). Dimensional change of $\mathrm{H}-451 / \mathrm{H}-429$ graphite, axial

orientation, as a function of fast neutron fluence at

irradiation temperatures of $650^{\circ}$ and $850^{\circ} \mathrm{C}$. . . . . . . . 5-33

5-1(c). Dimensional change of $\mathrm{H}-451 / \mathrm{H}-429$ graphite, axial

orientation, as a function of fast neutron fluence at

irradiation temperatures of $950^{\circ}$ and $1150^{\circ} \mathrm{C}$. . . . . . 5-34

5-1(d). Dimensional change of $\mathrm{H}-451 / \mathrm{H}-429$ graphite, axial

orientation, as a function of fast neutron fluence at

irradiation temperatures of $1050^{\circ}, 1250^{\circ}$, and $1350^{\circ} \mathrm{C}$. . . 5-35

5-2(a). Dimensional change of $\mathrm{H}-451 / \mathrm{H}-429$ graphite, radial

orientation; as a function of fast neutron fluence at

irradiation temperatures of $550^{\circ}$ and $750^{\circ} \mathrm{C}$. . . . . . 5-36

5-2(b). Dimensional change of $\mathrm{H}-451 / \mathrm{H}-429$ graphite, radial

orientation, as a function of fast neutron fluence at

irradiation temperatures of $650^{\circ}$ and $850^{\circ} \mathrm{C}$. . . . . . . 5-37

5-2(c). Dimensional change of $\mathrm{H}-451 / \mathrm{H}-429$ graphite, radial

orientation, as a function of fast neutron fluence at

irradiation temperatures of $950^{\circ}$ and $1150^{\circ} \mathrm{C}$. . . . . . . 5-38

5-2(d). Dimensional change of $\mathrm{H}-451 / \mathrm{H}-429$ graphite, radial

orientation, as a function of fast neutron fluence at

irradiation temperatures of $1050^{\circ}, 1250^{\circ}$, and $1350^{\circ} \mathrm{C}$. . . 5-39

5-3. Design curves for irradiation-induced axial dimensional change in $\mathrm{H}-451 / \mathrm{H}-429$ graphite . . . . . . . . . . . 5-41.

5-4. Design curves for irradiation-induced radial dimensional change in $\mathrm{H}-451 / \mathrm{H}-429$ graphite . . . . . . . . . . . . 5-42

5-5. Dimensional change in H-451 graphite for non-isothermal irradiation . . . . . . . . . . . . . . . . . 5-44

6-1(a). Percent change in thermal expansivity $\left(22^{\circ}-500^{\circ} \mathrm{C}\right)$ for $\mathrm{H}-451$ and TS-1240 near-isotropic graphites as a function of fast neutron fluence at irradiation temperatures of $590^{\circ}$ to $1060^{\circ} \mathrm{C}$............... 6-5

6-1(b). Percent change in thermal expansivity $\left(22^{\circ}-500^{\circ} \mathrm{C}\right)$ for $\mathrm{H}-451$ and TS- 1240 near-isotropic graphites as a function of fast neutron fluence at irradiation temperatures of $1105^{\circ}$ to $1500^{\circ} \mathrm{C}$. . . . . . . . . . . . . 6-6

6-2. Percent change in thermal expansivity $\left(22^{\circ}-500^{\circ} \mathrm{C}\right)$ for H-327 needle-coke graphite as a function of fast neutron fluence .................. 6-7 


\section{FIGURES (continued)}

\begin{tabular}{|c|c|c|c|}
\hline & $\begin{array}{l}\text { Observed thermal conductivity of } \mathrm{H}-451 \text { and TS-1240 near- } \\
\text { isotropic graphites at irradiation temperature as a } \\
\text { function of fast neutron fluence. . . . . . . . . . }\end{array}$ & . & $6-14$ \\
\hline $6-4$ & 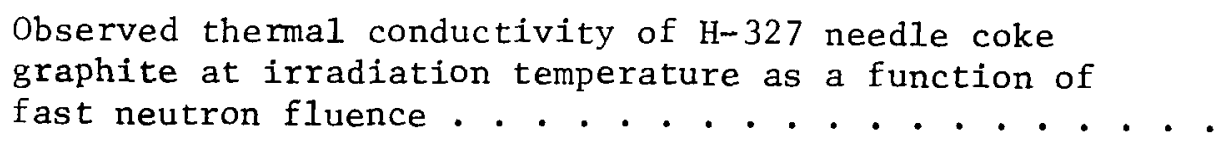 & . & $6-15$ \\
\hline $6-5$ & $\begin{array}{l}\text { Calculated thermal conductivity of } \mathrm{H}-451 \text { and } \mathrm{TS}-1240 \\
\text { near-isotropic graphites at irradiation temperature as } \\
\text { a function of irradiation temperature . . . . . . . . . . . }\end{array}$ & . . & $6-18$ \\
\hline $6-6$ & $\begin{array}{l}\text { Calculated thermal conductivity of } \mathrm{H}-327 \text { needle coke } \\
\text { graphite at irradiation temperature as a function of } \\
\text { irradiation temperature . . . . . . . . . . . . . . . . . . }\end{array}$ & . . & $6-19$ \\
\hline $7-1$ & $\begin{array}{l}\text { Observed tensile strength and Young's modulus of } \mathrm{H}-451 \\
\text { near-isotropic graphite as functions of fast neutron } \\
\text { fluence . . . . . . . . . . . . . . . . . . . . . . . }\end{array}$ & - . & $7-7$ \\
\hline $7-2$ & $\begin{array}{l}\text { Observed tensile strength and Young's modulus of TS-1240 } \\
\text { near-isotropic graphite as functions of fast neutron } \\
\text { fluence . . . . . . . . . . . . . . . . . . . . . . . . . . }\end{array}$ & & $7-8$ \\
\hline $3(a)$. & $\begin{array}{l}\text { Observed tensile strength and Young's modulus of } \mathrm{H}-327 \\
\text { needle coke graphite as functions of fast neutron fluence } \\
\text { at irradiation temperatures of } 640^{\circ} \text { to } 900^{\circ} \mathrm{C} . . . . . . \text {. . }\end{array}$ & & $7-9$ \\
\hline (b). & $\begin{array}{l}\text { Observed tensile strength and Young's modulus of } \mathrm{H}-327 \\
\text { needle coke graphite as functions of fast neutron fluence } \\
\text { at irradiation temperatures of } 940^{\circ} \text { to } 1200^{\circ} \mathrm{C} \text {. . . . . . . . }\end{array}$ & & $7-10$ \\
\hline 1. & $\begin{array}{l}\text { Calculated curves for the percent increase in Young's } \\
\text { modulus of near-isotropic graphites as a function of fast } \\
\text { neutron fluence and irradiation temperature . . . . . . . }\end{array}$ & & $8-3$ \\
\hline & $\begin{array}{l}\text { Calculated curves for the percent increase in tensile } \\
\text { strength of near-isotropic graphites as a function of } \\
\text { fast neutron fluence and irradiation temperature... }\end{array}$ & & \\
\hline
\end{tabular}

\section{TABLES}

3-1. Graphites and other materials irradiated in capsule OG-2 . . 3-2

4-1. Mean temperatures and fluences in capsule OG-2 . . . . . . 4-4

5-1. Dimensional change data, H-429, axial direction, after

irradiation in capsules $\mathrm{GEH}-422,452,482$. . . . . . . 5-2

5-2. Dimensional change data, H-429, axial direction, after irradiation in capsule $\mathrm{OG}-1$. . . . . . . . . . . 5-4

5-3. Dimensional change data, H-429, axial direction, after irradiation in capsule $\mathrm{OG}-2$. . . . . . . . . . 5-5 
TABLES (continued)

5-4. Dimensional change data, H-451, axial direction, after irradiation in capsule $0 G-1$. . . . . . . . . . 5-6

5-5. Dimensional change data, $\mathrm{H}-451$, axial direction, after irradiation in capsule $\mathrm{OG} 2$. . . . . . . . . . 5-15

5-6. Dimensional change data, $\mathrm{H}-429$, radial direction, after irradiation in capsules GEH-422, 452, 482 . . . . . . . . . 5-19

5-7. Dimensional change data, $\mathrm{H}-429$, radial direction, after irradiation in capsule $0 \mathrm{G}-1$. . . . . . . . . . . 5-21

5-8. Dimensional change data, $\mathrm{H}-429$, radial direction, after irradiation in capsule $\mathrm{OG}-2$. . . . . . . . . . 5-22

5-9. Dimensional change data, H-451, radial direction, after irradiation in capsule $\mathrm{OG}-1$. . . . . . . . . . . 5-23

5-10. Dimensional change data, $\mathrm{H}-451$, radial direction, after irradiation in capsule $0 G-2$. . . . . . . . . . 5-30

5-11. Comparison of dimensional changes in midlength center (MLC) and midlength edge (MLE) H-451 specimens . . . . . . . . . 5-45

5-12. Dimensional change data, TS-1240, axial direction, after irradiation in capsule $\mathrm{OG}-2$. . . . . . . . . . . . 5-46

5-13. Dimensional change data, TS-1240, radial direction, after irradiation in capsule $\mathrm{OG}-2$. . . . . . . . . . . . 5-48

5-14. Dimensional change data, $P_{3}$ JHAN, axial direction, after irradiation in capsule $O G-1$. . . . . . . . . . . 5-49

5-15. Dimensional change data, P3JHAN, axial direction, after irradiation in capsule $0 G-2$. . . . . . . . . . . . 5-51

5-16. Dimensional change data, $\mathrm{P}_{3} \mathrm{JHAN}$, radial direction, after irradiation in capsule $\mathrm{OG}-1$. . . . . . . . . . . . . 5-52

5-17. Dimensional change data, $\mathrm{P}_{3} \mathrm{JHAN}$, radial direction, after irradiation in capsule $\mathrm{OG}-2$. . . . . . . . . . . 5-54

5-18. Dimensional change data, H-327, axial direction, after irradiation in capsules $\mathrm{OG}-1$ and $\mathrm{OG}-2$. . . . . . . . . 5-56

5-19. Dimensional change data, $\mathrm{H}-327$, radial direction, after irradiation in capsules $\mathrm{OG}-1$ and $\mathrm{OG}-2$. . . . . . . . 5-62

5-20. Dimensional change data, treated $\mathrm{H}-451$ graphites, axia1 direction, after irradiation in capsule OG-2 . . . . . . 5-65

5-21. Dimensional change data, miscellaneous graphites, axia1 direction, after irradiation in capsule OG-2 . . . . . . . 5-66

5-22. Dimensional change data, miscellaneous graphites, radial direction, after irradiation in capsule OG-2 . . . . . . 5-68

5-23. Dimensional changes in carbon insulating materials irradiated in capsules $0 G-1$ and $O G-2$. . . . . . . . . . . 5-70 


\section{TABLES (continued)}

6-1. Thermal expansivity of $\mathrm{H}-429$ and $\mathrm{H}-451$ graphite specimens irradiated in capsule $O G-2$. . . . . . . . . . . . 6-2

6-2. Thermal expansivity of TS-1240 graphite specimens irradiated in capsule $\mathrm{OG}-2$............... 6-3

6-3. Thermal expansivity of H-327 graphite specimens irradiated in capsule $\mathrm{OG}-2$. . . . . . . . . . . . . . 6-4

6-4. Thermal conductivity of $\mathrm{H}-451$ graphite specimens irradiated in capsule $\mathrm{OG}-2$. . . . . . . . . . . . . . . 6-9

6-5. Thermal conductivity of TS -1240 graphite specimens irradiated in capsule $\mathrm{OG}-2$. . . . . . . . . . . . . . . . 6-11

6-6. Thermal conductivity of H-327 graphite specimens irradiated in capsule $\mathrm{OG}-2$. . . . . . . . . . . . . . . 6-13

6-7. Thermal conductivity parameters for irradiated graphites . . 6-17

7-1. Tensile properties of H-451 graphite specimens irradiated in capsule $\mathrm{OG}-2$. . . . . . . . . . . . . . 7-2

7-2. Tensile properties of TS -1240 graphite specimens irradiated in capsule $\mathrm{OG}-2$. . . . . . . . . . . . . . 7-3

7-3. Tensile properties of H-327 graphite specimens irradiated in capsule $\mathrm{OG} 2$. . . . . . . . . . . . . . . . 7-5

7-4. Summary of mechanical property data for graphites irradiated in capsules $\mathrm{OG}-1$ and $\mathrm{OG}-2$. . . . . . . . . . . . 7-11 


\section{INTRODUCTION}

1.1. SCOPE OF REPORT

This report describes the results of dimensional, thermal expansivity, thermal conductivity, Young's modulus, and tensile strength measurements on specimens of nuclear graphites irradiated in capsule OG-2. Details of the fabrication and assembly of the capsule are given in Ref. 1, and the capsule operating history is detailed in Ref. 2. A complete list of the specimens, based on their location in the capsule, is contained in Ref. 3.

\subsection{FAST FLUENCE UNITS}

A change has been made in the units used for reporting fast neutron fluences. Earlier reports on this program, including the topical report on the preceding capsule OG-1 (Ref. 4), presented integrated fluences of neutrons with energies greater than $0.18 \mathrm{MeV}$ in the water-moderated test reactors where the capsules were irradiated $\left[\mathrm{n} / \mathrm{cm}^{2}(E>0.18 \mathrm{MeV})_{\mathrm{ORR}}\right]$. In the present report, the fluences are converted to the following units:

1. Fluence of neutrons with energies greater than $0.18 \mathrm{MeV}$ in a typical High-Temperature, Gas-Cooled Reactor (HTGR) spectrum $\left[\mathrm{n} / \mathrm{cm}^{2}(\mathrm{E}>0.18 \mathrm{MeV})_{\mathrm{HTGR}}\right]$. This is the unit that will be used in future HTGR design work.

2. Equivalent fission fluence for graphite damage ( $\left.\mathrm{n} / \mathrm{cm}^{2}, \mathrm{EFFGD}\right)$. This is the unit recommended by an international working group sponsored by the International Atomic Energy Authority (Ref. 5) and forms the basis of the ASTM recommended practice for reporting dosimetry results on graphite. 
Conversions are based on the number of carbon atoms displaced, calculated based on the Thompson and Wright model, according to which $1 \mathrm{n} / \mathrm{cm}^{2}$ (EFFGD) results in $720 \times 10^{-24}$ displaced carbon atoms per atom. The appropriate conversion factors for the $\mathrm{C}-3$ position of the Oak Ridge Reactor (ORR) are:

$$
\begin{aligned}
\text { Fluence }(E>0.18 \mathrm{MeV})_{\text {HTGR }} & =0.894 \times \text { Fluence }(E>0.18 \mathrm{MeV})_{\text {ORR }} \\
\text { Fluence }(E F F G D) & =1.118 \times \text { Fluence }(E>0.18 \mathrm{MeV})_{\text {ORR }} \\
\text { Fluence }(E F F G D) & =1.25 \times \text { Fluence }(E>0.18 \mathrm{MeV})_{\text {HTGR }}
\end{aligned}
$$

At points in the text where no fluence units are specified, $n / \mathrm{cm}^{2}$ (E > $0.18 \mathrm{MeV})_{\text {HTGR }}$ should be understood.

Further details of the dosimetry are given in Section 4.3 . 


\section{TEST DESCRIPTION AND OBJECTIVES}

Capsule OG-2 was the second in a series of graphite irradiation capsules which are being operated to obtain design basis data on the effects of neutron irradiation on the physical properties of candidate graphites for HTGR replaceable fuel and reflector elements. The Fort St. Vrain (FSV) HTGR used a needle coke graphite (grade H-327), while current designs for large HTGRs favor the use of a near-isotropic graphite. Nearisotropic petroleum-coke-based graphites, extruded in 17- to 18-in.-diameter logs, are now receiving highest priority.

In the OG capsule series, the operating temperatures in nine out of the 10 crucibles in the capsule span the range where significant neutron exposure occurs in HTGRs $\left(600^{\circ}\right.$ to $\left.1200^{\circ} \mathrm{C}\right)$, and the tenth crucible operates at $1400^{\circ} \mathrm{C}$ to provide test data at a higher temperature. Capsule OG-2 had a thermal design identical to those of the preceding (OG-1) and succeeding (OG-3) capsules, and many specimens were transferred from capsule to capsule after nondestructive measurements in order to provide data at several increments of fluence. 


\section{MATERIALS INVESTIGATED}

The materials investigated and the numbers of specimens are summarized in Table 3-1.

A major objective of the experiment was to establish the data base for the irradiation-induced property changes in H-451 near-isotropic petroleumcoke-based graphite made by Great Lakes Carbon Company. About half the irradiation space was allocated to specimens of $\mathrm{H}-451$ or its subsized prototype grade $\mathrm{H}-429$. Most of the $\mathrm{H}-451$ specimens had been irradiated in capsule $O G-1$, and all the H-429 specimens had been irradiated in capsules GEH-13-422 and OG-1. The accumulated fluence on the H-429 specimens in the higher-temperature crucibles now exceeds the end-of-life fluence for current HTGR designs.

Another objective was to complete the collection of data on H-327 needle coke graphite, which was used in the construction of the FSV HTGR. Two hundred specimens of $\mathrm{H}-327$, most of which had been previously irradiated in capsule GEH-13-422 or OG-1, were included in the experiment.

Also included in capsule OG-2 were 134 virgin specimens of TS-1240 graphite, a near-isotropic petroleum-coke-based graphite made by Union Carbide Corporation. Other materials included a pitch-coke-based graphite (Pechiney grade $\mathrm{P}_{3} \mathrm{JHAN}$ ) and miscellaneous graphites and carbons of general interest to the program.

Three specimen configurations were used: 0.2-in.-diameter by 0.45-in.long cylinders for nondestructive dimensional and thermal expansivity determinations, 0.4-in.-diameter by 0.05-in.-thick disks for nondestructive thermal diffusivity measurements, and 0.25-in.-diameter by 0.9-in.-long cylinders for dimensional measurements and destructive tensile testing. 
TABLE 3-1

GRAPHITES AND OTHER MATERIALS IRRADIATED IN CAPSULE OG-2

\begin{tabular}{|c|c|c|c|c|c|c|c|c|}
\hline \multirow[b]{2}{*}{ Designation } & \multirow{2}{*}{$\begin{array}{l}\text { No. of } \\
\text { Specimens }\end{array}$} & \multirow[b]{2}{*}{. Type } & \multirow{2}{*}{\begin{tabular}{rl|} 
GA & Log \\
Book & Number
\end{tabular}} & \multirow{2}{*}{$\begin{array}{l}\text { Form \& Dimensions } \\
\text { of Parent Sample }\end{array}$} & \multirow[b]{2}{*}{ Source $(a)$} & \multicolumn{3}{|c|}{ Raw Materials } \\
\hline & & & & & & Filler & Binder & Impregnant \\
\hline$\underset{\mathrm{H}-451}{\text { Graphite }}$ & 428 & $\begin{array}{l}\text { Extruded, near } \\
\text { isotropic }\end{array}$ & $\begin{array}{l}5651-28 \\
5651-86\end{array}$ & $\begin{array}{l}\text { 18-in.-diam. x 34-in.- } \\
\text { long Jog }\end{array}$ & GLCC & $\begin{array}{l}\text { Near-isotropic } \\
\text { petroleum coke }\end{array}$ & $\begin{array}{l}\text { Coal tar } \\
\text { pitch }\end{array}$ & $\begin{array}{l}\text { Petroleum } \\
\text { pitch }\end{array}$ \\
\hline $\mathrm{H}-429$ & 69 & $\begin{array}{l}\text { Extruded, near } \\
\text { isotropic }\end{array}$ & 4974-104-A & $\begin{array}{l}\text { 9-in.-diam. x 8-in.- } \\
\text { long log }\end{array}$ & GLCC & $\begin{array}{l}\text { Near-isotropic } \\
\text { petroleum coke }\end{array}$ & $\begin{array}{l}\text { Coal tar } \\
\text { pitch }\end{array}$ & $\begin{array}{l}\text { Coal tar } \\
\text { pitch }\end{array}$ \\
\hline $\mathrm{TS}-1240$ & 134 & $\begin{array}{l}\text { Extruded, near } \\
\text { isotropic }\end{array}$ & $5651-73$ & $\begin{array}{l}\text { 18-in.-diam. x 34-in.- } \\
\text { long } \log \end{array}$ & UCC & $\begin{array}{l}\text { Near-isotropic } \\
\text { petroleum coke }\end{array}$ & & \\
\hline $\mathrm{H}-327$ & 204 & $\begin{array}{l}\text { Extruded, } \\
\text { anisotropic }\end{array}$ & $4974-3$ & $\begin{array}{l}\text { 18-in.-diam. x 34-in.- } \\
\text { long log }\end{array}$ & GLCC & Needle coke & $\begin{array}{l}\text { Coal tar } \\
\text { pitch }\end{array}$ & $\begin{array}{l}\text { Coal tar } \\
\text { pitch }\end{array}$ \\
\hline $\mathrm{P}_{3}$ JHAN & 66 & $\begin{array}{l}\text { Extruded, near } \\
\text { isotropic }\end{array}$ & $5651-53$ & $\begin{array}{l}8-i n \cdot x \text {-in. } x \text { 8-in. } \\
\text { cube }\end{array}$ & Pechiney & $\begin{array}{l}\text { Near-isotropic } \\
\text { coal tar pitch } \\
\text { coke }\end{array}$ & $\begin{array}{l}\text { Coal tar } \\
\text { pitch }\end{array}$ & $\begin{array}{l}\text { Coal tar } \\
\text { pitch }\end{array}$ \\
\hline 2020 & 120 & $\begin{array}{l}\text { Isostatic } \\
\text { molded, fine } \\
\text { grained }\end{array}$ & $5651-56$ & $\begin{array}{l}\text { 18-in.-diam. x 6-in.- } \\
\text { long slab }\end{array}$ & SCC & Petroleum coke & (b) & (b) \\
\hline $\mathrm{H}-328$ & 1 & $\begin{array}{l}\text { Molded, near } \\
\text { isotropic }\end{array}$ & -- & $\begin{array}{l}\text { 17-in.-diam. x 34-in.- } \\
\text { long log }\end{array}$ & GLCC & Gilso coke & $\begin{array}{l}\text { Coal tar } \\
\text { pitch }\end{array}$ & $\begin{array}{l}\text { Coal tar } \\
\text { pitch }\end{array}$ \\
\hline$H-430$ & 1 & $\begin{array}{l}\text { Molded, near } \\
\text { isotropic }\end{array}$ & -- & $\begin{array}{l}8.6 \text {-in.-diam. } x \\
7.5 \text {-in.-long log }\end{array}$ & GLCC & $\begin{array}{l}\text { Near-isotropic } \\
\text { petroleum coke }\end{array}$ & $\begin{array}{l}\text { Coal tar } \\
\text { pitch }\end{array}$ & (b) \\
\hline $\mathrm{IH}-451$ & 14 & $\begin{array}{l}\text { Extruded, near } \\
\text { isotropic }\end{array}$ & $5948-101$ & $\begin{array}{l}\text { 18-in.-diam. x 34-in.- } \\
\text { long } 10 g\end{array}$ & $\mathrm{GLCC} / \mathrm{GA}$ & $\begin{array}{l}\text { Near-isotropic } \\
\text { petroleum coke }\end{array}$ & $\begin{array}{l}\text { Coal tar } \\
\text { pitch }\end{array}$ & $\begin{array}{l}\text { Additional } \\
\text { impregnation } \\
\text { done at GA }\end{array}$ \\
\hline $\mathrm{H}-451 \mathrm{C}$ & 12 & $\begin{array}{l}\text { Extruded, near } \\
\text { isotropic }\end{array}$ & $5948-14$ & $\begin{array}{l}\text { 18-in.-diam. x 34-in.- } \\
\text { long log }\end{array}$ & $\mathrm{GLCC} / \mathrm{GA}$ & $\begin{array}{l}\text { Near-isotropic } \\
\text { petroleum coke }\end{array}$ & $\begin{array}{l}\text { Coal tar } \\
\text { pitch }\end{array}$ & $\begin{array}{l}\text { Samples } \\
\text { taken from } \\
\text { cure-in- } \\
\text { place } \\
\text { experiments }\end{array}$ \\
\hline 565161 & 16 & Experimental & $5651-61$ & (b) & ORNL & (b) & (b) & (b) \\
\hline
\end{tabular}


TABLE 3-1 (Continued)

\begin{tabular}{|c|c|c|c|c|c|c|}
\hline Designation & $\begin{array}{l}\text { No. of } \\
\text { Specimens }\end{array}$ & Type & $\begin{aligned} \text { GA } & \text { Log } \\
\text { Book } & \text { Number }\end{aligned}$ & $\begin{array}{l}\text { Form \& Dimensions } \\
\text { of Parent Sample }\end{array}$ & Source ${ }^{(a)}$ & Description \\
\hline $\begin{array}{l}\text { Other } \\
\text { Materials }\end{array}$ & & & & & & \\
\hline $\mathrm{B}_{4} \mathrm{C}-\mathrm{G}$ & 18 & $\begin{array}{l}\text { Extruded } \\
\text { boronated } \\
\text { graphite }\end{array}$ & $\begin{array}{l}5646-13 \\
5646-91\end{array}$ & $\begin{array}{l}0.30-\text { in. o.d. } \times 10-\text { in. } \\
\text { long rods } \\
0.25 \text {-in. o.d. } \times 0.9- \\
\text { in.-1ong rods }\end{array}$ & $\begin{array}{l}\text { GLCC } \\
\text { (c) }\end{array}$ & $\begin{array}{l}\text { FSV lumped burnable poison } \\
\text { Oxidation-resistant shield compacts }\end{array}$ \\
\hline MATRIX & 4 & $\begin{array}{l}\text { Binder } \\
\text { residue }\end{array}$ & - & (b) & GA & $\begin{array}{l}\text { Coke residue from pitch binder in } \\
\text { large HTGR fuel rod matrix }\end{array}$ \\
\hline IMPCBN & 1 & $\begin{array}{l}\text { Impregnant } \\
\text { carbon }\end{array}$ & -- & (b) & GA & Carbon residue from impregnant \\
\hline GLSYCN & 18 & Glassy carbons & - & (b) & GA & Model carbons \\
\hline PyC & 13 & $\begin{array}{l}\text { Pyrolytic } \\
\text { carbon }\end{array}$ & -- & (b) & GA & Fuel particle coating material \\
\hline $\mathrm{SiC}$ & 4 & SiC coatings & $6041-7$ & (b) & GA & Fuel particle coating material \\
\hline PALAR & 1 & Insulation & - & (b) & (b) & Palarite insulation \\
\hline FPA & 1 & Insulation & -- & (b) & (b) & Carbon foam insulation \\
\hline
\end{tabular}

(a). Great Lakes Carbon Company (GLCC); Union Carbide Corporation (UCC); Stackpole Carbon Company (SCC); Pechiney (France); General Atomic Company (GA); Oak Ridge National Laboratory (ORNL).

(b) Unknown.

(c) Rods fabricated by GLCC, GA, Norton Company, Carborundum Company. 
Only two batches of $\mathrm{H}-451$ tensile specimens were tested to failure; the majority were reencapsulated in capsule $O G-3$ for additional exposure. All the H-327 tensile specimens were tested, with the exception of two batches from a low-fluence crucible which were held for reirradiation in capsule OG-3. Half of the TS-1240 tensile specimens were tested, and the remainder were held for reirradiation in capsule OG-3. 


\section{CAPSULE OPERATING CONDITIONS}

\subsection{DESCRIPTION OF CAPSULE}

Capsule OG-2 was a fully instrumented, controlled-temperature graphite irradiation capsule irradiated in the $\mathrm{C}-3$ position of the ORR. Irradiation started on July 17, 1974 and terminated on January 5, 1975 for a capsule cumulative power of 3886 MWd and an accumulated irradiation time at power of $1.12 \times 10^{7} \mathrm{sec}$.

The capsule contained two cells, each with its own sweep-gas system. Adjustment of the helium-argon ratio in the sweep gas allowed independent temperature control in the upper and lower cells. The cells contained a total of 10 graphite crucibles approximately 2.25 in. in diameter by 2 in. high (except for crucible No. 3, which was 3 in. high), which were drilled with holes to act as specimen holders. The graphite crucibles were contained in Inconel 600 containment cans, which were housed in an annular aluminum thermal bond inside two 304 stainless steel containment tubes. The center-line temperatures of crucible 3 in cell 1 and crucible 7 in cell 2 were maintained at $1000^{\circ} \mathrm{C}$ during reactor operation by adjustment of the gas mixtures. The capsule contained 24 Chromel-Alumel thermocouples and six tungsten-rhenium thermocouples. All crucibles had one or more thermocouples located near the center line, and all except two had one or more thermocouples at the periphery. A schematic diagram of the capsule layout and thermocouple locations is shown in Fig. 4-1. Three sets of in situ decalibration data on the tungsten-rhenium thermocouples were obtained by locating pairs of Chromel-Alumel $(K)$ and tungsten-rhenium (W) thermocouples in the same holes in the crucibles of cell 1, which operated at or below $1000^{\circ} \mathrm{C}$. Reliable readings were obtained from all thermocouples until 58\% of life, when thermocouples $\mathrm{W} 18$ and $\mathrm{W} 19$ failed at the start of a cycle. Thermocouple W- 8 became intermittent at $68 \%$ of life. The remaining 27 thermocouples operated for the full capsule life. 


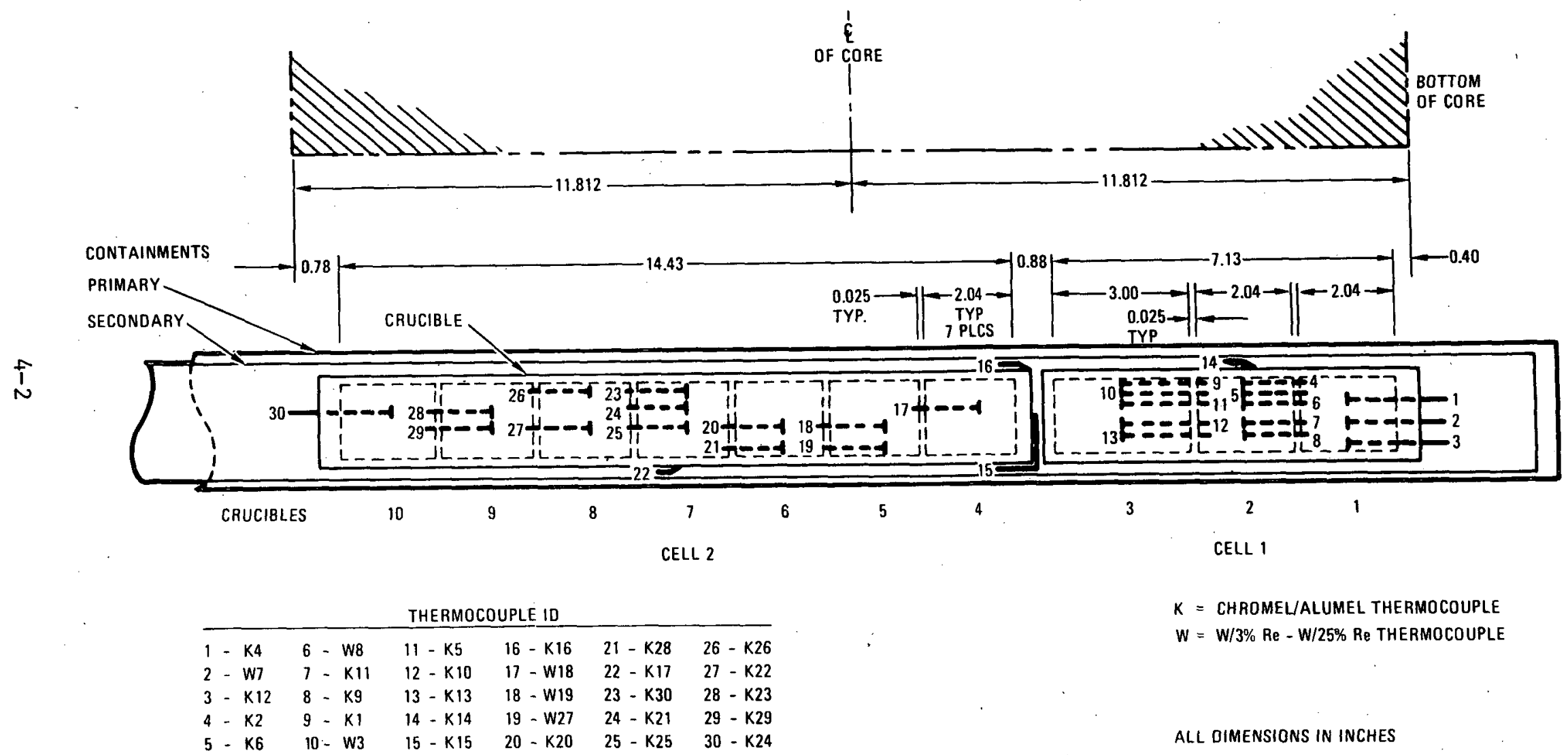

Fig. 4-1. Schematic layout of capsule OG-2 with thermocouple locations 


\subsection{OPERATING TEMPERATURES}

Recorded temperature histories and details of the procedures used to analyze the thermocouple operating records are given in Ref. 2. The neutroninduced decalibration factor for the tungsten-rhenium thermocouples used to monitor the temperatures in high-temperature crucibles 4 and 5 was taken from the W3/K1 thermocouple pair in the closest crucible (No. 3). The factor relating true operating temperature, $T_{t}$, and the indicated temperature, $T_{i}$, of the tungsten-rhenium thermocouples in crucibles 4 and 5 was taken to be

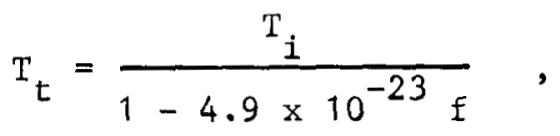

where $f$ is the thermal fluence $\left(n / \mathrm{cm}^{2}, E<0.17 \mathrm{eV}\right)$.

Two crucibles ( 4 and 10) had a thermocouple near the center line but not at the periphery. Estimates of the radial temperature gradients in these crucibles were made from the observed temperature gradients in adjacent crucibles, taking into account the gamma heating rates. After the failure of thermocouples $W 18$ and $W 19$, the temperatures at these locations were estimated from thermocouple W27, using the ratios established up to the point of failure.

The average on-test temperatures at the center line and outer thermocouple locations are listed in Table 4-1. Long-term drift in the centerline temperatures was negligible, but the irradiation-induced decrease in graphite thermal conductivity caused a progressive increase in the radial temperature gradients in the crucibles. This resulted in a downward drift in the outside temperatures over the life of the capsule averaging $30^{\circ} \mathrm{C}$. In addition to long-term drift in the outer temperatures, changing gamma heating rates resulting from control rod bank travel superimposed a sawtooth pattern on the operating temperatures (with the exception of controlled crucibles 3 and 7 ). The upper limit of the fluctuations (defined as the 
TABLE 4-1

MEAN TEMPERATURES AND FLUENCES IN CAPSULE OG-2

\begin{tabular}{|c|c|c|c|c|c|c|c|c|c|c|c|c|}
\hline \multirow{3}{*}{$\begin{array}{c}\text { Crucible } \\
\text { Number }\end{array}$} & \multicolumn{2}{|c|}{ Fast Fluence $\left(x 10^{-21}\right)$} & \multirow{3}{*}{$\begin{array}{l}\text { Thermal. Fluence } \\
\left(\times 10^{-21}\right)\left(\mathrm{n} / \mathrm{cm}^{2}\right)\end{array}$} & \multirow{3}{*}{$\begin{array}{c}\text { Nominal } \\
\text { Center } \\
\text { Line } \\
\text { Design } \\
\text { Temperature } \\
\left({ }^{\circ} \mathrm{C}\right)\end{array}$} & \multicolumn{6}{|c|}{$\begin{array}{c}\text { Operating Temperature at Thermocouple } \\
\text { Location }\left({ }^{\circ} \mathrm{C}\right)\end{array}$} & \multirow{2}{*}{\multicolumn{2}{|c|}{$\begin{array}{c}\text { Mean Operating } \\
\text { Temperature in } \\
\text { OG-1 Capsule }\end{array}$}} \\
\hline & \multirow{2}{*}{$\begin{array}{c}\mathrm{n} / \mathrm{cm}^{2} \\
(\mathrm{E}>0.18 \mathrm{MeV})_{\mathrm{HTGR}}\end{array}$} & \multirow{2}{*}{$\begin{array}{c}\mathrm{n} / \mathrm{cm}^{2} \\
(\mathrm{EFFGD})\end{array}$} & & & \multicolumn{3}{|c|}{ Center } & \multicolumn{3}{|c|}{ Outer } & & \\
\hline & & & & & Mean & $\operatorname{Max}{ }^{(a)}$ & Min. (a) & Mean & $\operatorname{Max} .^{(a)}$ & Min. (a) & Center & Outer \\
\hline 1 & 1.7 & 2.1 & 1.2 & 700 & 687 & 713 & 667 & 602 & 626 & 580 & 657 & 558 \\
\hline 2 & 2.2 & 2.8 & 1.4 & 800 & 778 & 802 & 758 & 664 & 688 & 641 & 754 & 610 \\
\hline 3 & 2.7 & 3.4 & 1.6 & 1000 & 998 & 1014 & 983 & 907 & 930 & 884 & 993 & 887 \\
\hline 4 & 2.9 & 3.7 & 1.8 & 1200 & 1251 & 1333 & 1158 & 1090 & -- & -- & 1172 & 1024 \\
\hline 5 & 2.9 & 3.6 & 1.8 & 1400 & 1387 & 1449 & 1319 & 1316 & 1380 & 1255 & 1395 & 1327 \\
\hline 6 & 2.7 & 3.4 & 1.7 & 1100 & 1080 & 1110 & 1047 & 937 & 973 & 897 & 1078 & 938. \\
\hline 7 & 2.5 & 3.1 & 1.5 & 1000 & 997 & 1016 & 983 & 907 & 932 & 881 & 992 & 884 \\
\hline 8 & 2.1 & 2.7 & 1.2 & 900 & 934 & 970 & 905 & 835 & 866 & 808 & 928 & 836 \\
\hline 9 & 1.7 & 2.1 & 0.9 & 800 & 830 & 883 & 793 & 752 & 794 & 713 & 860 & 753 \\
\hline 10 & 1.2 & 1.5 & 0.6 & 600 & 640 & 706 & 602 & 580 & -- & -- & 650 & 597 \\
\hline
\end{tabular}

(a) Maximum and minimum temperatures denote the range within which the temperatures remained for $90 \%$ of the time the reactor was at power. 
temperature below which the thermocouple operated for $95 \%$ of the on-test time) and the lower limit (the temperature above which the thermocouple operated for $95 \%$ of the on-test time) are listed in Table 4-1.

For comparison, Table 4-1 also shows the nominal center-line design temperatures and the mean temperatures recorded during the operation of the preceding capsule, OG-1, which had an identical thermal design. Agreement between the two capsules, and with the design temperatures, is very good.

The mean operating temperatures of individual specimens were calculated from the center-1ine and outer temperatures of the crucible, assuming a parabolic temperature distribution along the radius.

\subsection{NEUTRON FLUENCE}

Dosimetry for the capsule was obtained from vanadium $-0.216 \%$ cobalt, vanadium - $0.52 \%$ iron ( $88.25 \%$ enriched in $\mathrm{Fe}^{54}$ ), and titanium wires contained in quartz vials placed in grooves in the aluminum thermal bond. Wires were placed at seven axial locations and four circumferential positions $90^{\circ}$ apart. After irradiation wires from all positions except one were recovered and analyzed by gamma-ray spectroscopy. Fast $\left[(E:>0.18 \mathrm{MeV})_{\mathrm{ORR}}\right]$ and thermal fluences were calculated assuming activation cross sections of $46.56 \mathrm{mb}$ for the $\mathrm{Fe}^{54}(\mathrm{n}, \mathrm{p}) \mathrm{Mn}^{54}$ reaction, $6.61 \mathrm{mb}$ for the $\mathrm{Ti}^{46}(\mathrm{n}, \mathrm{p}) \mathrm{Sc}^{46}$ reaction, and $36.8 \mathrm{~b}$ for the $\mathrm{Co}^{59}(\mathrm{n}, \gamma) \mathrm{Co}^{60}$ reaction. The fast fluences obtained from the four circumferential positions at a given axial location were averaged. Allowance was made for a $1.3 \%$ decrease in flux between the monitor location and specimen locations. The means of the iron and titanium data for fluence in $(E>0.18 \mathrm{MeV})_{O R R}$ were converted to fluence in $(E>0.18 \mathrm{MeV})_{\text {HTGR }}$ and equivalent fission fluence for graphite damage through conversion factors based on the number of displaced carbon atoms, using the Thompson and Wright model with $1 \mathrm{n} / \mathrm{cm}^{2}$ (EFFDG) causing $720 \times 10^{-24}$ displacements per atom. The following factors were used:

$$
\begin{aligned}
& \text { Fluence }(E>0.18 \mathrm{MeV})_{\text {HTGR }}=0.894 \times \text { Fluence }(E>0.18 \mathrm{MeV})_{O R R} \\
& \text { Fluence }(\text { EFFGD })=1.118 \times \text { Fluence }(E>0.18 \mathrm{MeV})_{O R R} .
\end{aligned}
$$


Fast and thermal fluences at each of the seven axial locations are plotted in Fig. 4-2 as a function of height above the bottom of the core. The corresponding fluences at the midpoint of each crucible are listed in Table 4-1. 


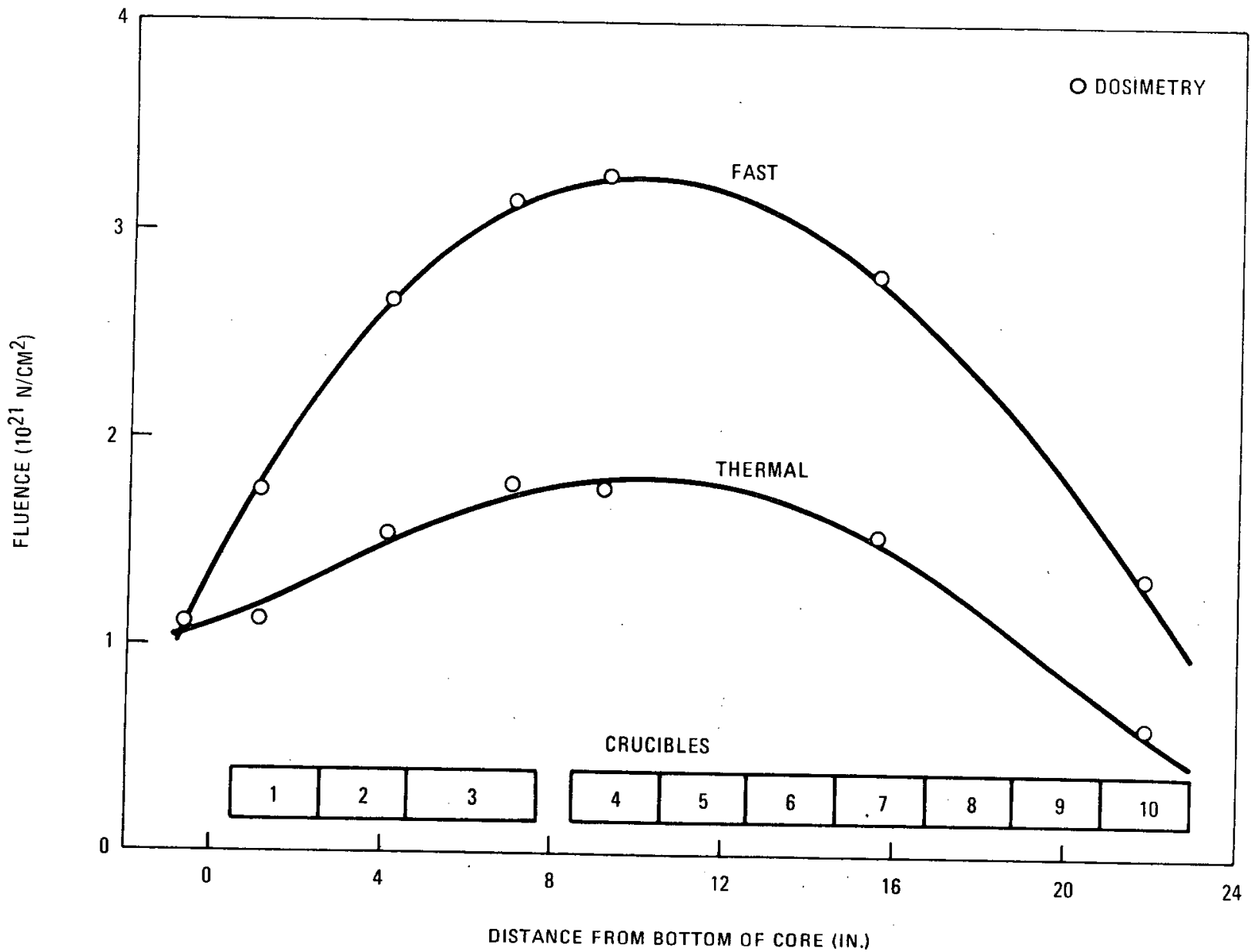
Fig. 4-2. Measured fast $(E>0.18 \mathrm{MeV})_{\mathrm{ORR}}$ and thermal fluence values for
the OG-2 experiment 


\section{EXPERIMENTAL RESULTS - DIMENSIONAL CHANGES}

\section{1. IRRADIATION-INDUCED DIMENSIONAL CHANGE IN NEAR-ISOTROPIC GRAPHITES}

The OG-2 experiment included specimens from four grades of nearisotropic nuclear graphite. Dimensional changes were measured on $69 \mathrm{H}-429$ and $394 \mathrm{H}-451$ graphite specimens taken from GLCC prototype logs. In addition, the dimensions of $98 \mathrm{TS}-1240$ and $66 \mathrm{P}_{3}$ JHAN graphite specimens were measured. All specimens were cylinders, either 0.20 in. in diameter by $0.45 \mathrm{in.}$ long or $0.25 \mathrm{in.}$ in diameter by $0.9 \mathrm{in.}$ long.

The total dimensional change of each specimen was calculated from the length of the specimen taken before any irradiation $\left(l_{0}\right)$ and the length of the cylinder following the OG-2 irradiation ( $l)$. The dimensional change was calculated as $\left(l-\ell_{0}\right) / l_{0}$ and the irradiation strain was calculated as $\ln \left(\ell / \ell_{0}\right)$. For total strains of less than $0.01 \mathrm{in.} / \mathrm{in} .,\left(\ell-\ell_{0}\right) / \ell_{0}=\ln \left(\ell / \ell_{0}\right)$. Where $\left(l-l_{0}\right) / l_{0}>0.01$, both values are given in the data tables presented herein. Each graphite specimen was assigned an average irradiation temperature and a mean fast neutron fluence based upon the specimen location in the OG-2 capsule crucibles as detailed earlier. For previously irradiated specimens, the total fast fluence was found by adding the OG-2 fluence to the previous fast fluence value. Average irradiation temperature was calculated using fluence-weighted temperatures for specimens subjected to multiple irradiations (i.e., for specimen 6500, $\mathrm{T}_{\text {avg }}=[(2.0 \times 660)+(1.7 \times 690)] / 3.7$ 6 675). All fast neutron fluences are in HTGR units $(\mathrm{E}>0.18 \mathrm{MeV})_{\text {HTGR }}$.

Data previously reported in ORR fluences have been restated in HTGR fluences and are presented, along with minor temperature corrections for the OG-1 capsule data and earlier H-429 data, in Tables 5-1 through 5-10. The $\mathrm{H}-451 / \mathrm{H}-429$ data are plotted in Figs. 5-1 and 5-2. The data plotted in Figs. 5-1 and 5-2 are grouped in $100^{\circ} \mathrm{C}$ intervals about the midpoint temperature (i.e., $950^{\circ} \mathrm{C}$ data include all data from $900^{\circ}$ to $999^{\circ} \mathrm{C}$ ). 
TABLE 5-1

DIMENSIONAL CHANGE DATA, H-429, AXIAL DIRECTION, AFTER IRRADIATION IN CAPSULES GEH-422, 452, 482

\begin{tabular}{|c|c|c|c|c|}
\hline $\begin{array}{c}\text { Record } \\
\text { No. }\end{array}$ & $\begin{array}{c}\text { Total } \\
\text { Fluence } \\
\left(10^{21} \mathrm{n} / \mathrm{cm}^{2}\right) \\
(\mathrm{E}>0.18 \mathrm{MeV})_{\text {HTGR }}\end{array}$ & $\begin{array}{r}\text { Irrad } \\
\text { Temp } \\
\left({ }^{\circ} \mathrm{C}\right)\end{array}$ & $\begin{array}{c}\text { Irrad } \\
\text { Strain } \\
\ln (\ell / \ell o) \\
\left(10^{-2}\right)\end{array}$ & $\begin{array}{c}\text { Dimensional } \\
\text { Change, } \\
\Delta \ell / \ell_{0} \\
(\%)\end{array}$ \\
\hline $\begin{array}{l}3624 \\
3600 \\
3601 \\
3602 \\
3603 \\
3604 \\
3605 \\
2444 \\
2448 \\
2452 \\
2473 \\
2489 \\
2492 \\
2500 \\
2522 \\
3700 \\
3724 \\
3701 \\
3702 \\
3703 \\
3704 \\
3705 \\
2537 \\
2594 \\
2605 \\
2606 \\
2612 \\
2653 \\
2659 \\
2667 \\
2671 \\
2673 \\
2680 \\
2648 \\
2649 \\
2642\end{array}$ & $\begin{array}{l}2.75 \\
2.75 \\
2.80 \\
2.90 \\
3.00 \\
3.07 \\
3.10 \\
0.27 \\
1 \\
0.27 \\
2.75 \\
2.75 \\
2.80 \\
2.90 \\
3.00 \\
3.07 \\
3.10 \\
0.73 \\
1 \\
0.73 \\
1.10 \\
1\end{array}$ & $\mid$ & $\begin{array}{l}-0.72 \\
-0.66 \\
-0.69 \\
-0.73 \\
-0.74 \\
-0.81 \\
-0.82 \\
+0.05 \\
+0.02 \\
+0.07 \\
+0.03 \\
+0.04 \\
+0.04 \\
+0.02 \\
+0.05 \\
-0.45 \\
-0.54 \\
-0.53 \\
-0.52 \\
-0.55 \\
-0.51 \\
-0.63 \\
-0.03 \\
-0.06 \\
-0.01 \\
-0.03 \\
-0.02 \\
-0.14 \\
-0.11 \\
-0.15 \\
-0.23 \\
-0.20 \\
-0.12 \\
-0.15 \\
-0.21 \\
-0.19\end{array}$ & \\
\hline
\end{tabular}


TABLE 5-1 (continued)

\begin{tabular}{|c|c|c|c|c|}
\hline $\begin{array}{c}\text { Record } \\
\text { No. }\end{array}$ & $\begin{array}{c}\text { Total } \\
\text { F1uence } \\
\left(10^{21} \mathrm{n} / \mathrm{cm}^{2}\right) \\
(\mathrm{E}>0.18 \mathrm{MeV})_{\text {HTGR }}\end{array}$ & $\begin{array}{r}\text { Irrad } \\
\text { Temp } \\
\left({ }^{\circ} \mathrm{C}\right)\end{array}$ & $\begin{array}{c}\text { Irrad } \\
\text { Strain } \\
\ln (\ell / \ell) \\
\left(10^{-2}\right)\end{array}$ & $\begin{array}{c}\text { Dimensional } \\
\text { Change, } \\
\Delta \ell / \ell \mathrm{l} \\
(\%)\end{array}$ \\
\hline $\begin{array}{l}2440 \\
2689 \\
2692 \\
2694 \\
2706 \\
2402 \\
2403 \\
2419 \\
2426 \\
2754 \\
2761 \\
2764 \\
2850 \\
2853 \\
2791 \\
2815 \\
2816 \\
2821 \\
2866 \\
2870 \\
2871 \\
2872 \\
2882\end{array}$ & $\begin{array}{l}\prod_{1.55}^{1.55} \\
2.20 \\
2.20 \\
2.65 \\
2.65\end{array}$ & \begin{tabular}{|}
$\mid 100$ \\
1275 \\
1275 \\
1325 \\
1325 \\
1475 \\
1475 \\
1500 \\
$\mid$
\end{tabular} & $\begin{array}{l}-0.26 \\
-0.28 \\
-0.21 \\
-0.23 \\
-0.28 \\
-1.08 \\
-0.80 \\
-0.97 \\
-1.18 \\
-1.06 \\
-1.22 \\
-1.36 \\
-1.86 \\
-1.91 \\
-1.83 \\
-2.08 \\
-1.85 \\
-1.84 \\
-1.98 \\
-2.36 \\
-2.19 \\
-2.00 \\
-2.22\end{array}$ & $\begin{array}{l}-1.17 \\
-1.05 \\
-1.21 \\
-1.35 \\
-1.84 \\
-1.89 \\
-1.81 \\
-2.05 \\
-1.83 \\
-1.82 \\
-1.96 \\
-2.33 \\
-2.16 \\
-1.98 \\
-2.19\end{array}$ \\
\hline
\end{tabular}


TABLE 5-2

DIMENSIONAL CHANGE DATA, H-429, AXIAL DIRECTION, AFTER IRRADIATION IN CAPSULE OG-1

\begin{tabular}{|c|c|c|c|c|c|c|c|c|c|}
\hline $\begin{array}{c}\text { Record } \\
\text { No. }\end{array}$ & $\begin{array}{l}\text { Previous } \\
\text { Record } \\
\text { No. }\end{array}$ & $\begin{array}{c}\text { Previous } \\
\text { F1uence } \\
\left(10^{21} \mathrm{n} / \mathrm{cm}^{2}\right) \\
(\mathrm{E}>0.18 \mathrm{MeV})_{\mathrm{HTGR}}\end{array}$ & $\begin{array}{l}\text { Previous } \\
\text { Temp } \\
\left({ }^{\circ} \mathrm{C}\right)\end{array}$ & $\begin{array}{c}\text { F1uence } \\
\text { This Irrad } \\
\left(10^{21} \mathrm{n} / \mathrm{cm}^{2}\right) \\
(\mathrm{E}>0.18 \mathrm{MeV})_{\text {HTGR }}\end{array}$ & $\begin{array}{l}\text { Temp } \\
\text { This } \\
\text { Irrad } \\
\left({ }^{\circ} \mathrm{C}\right)\end{array}$ & $\begin{array}{c}\text { Total } \\
\text { F1uence } \\
\left(10^{21} \mathrm{n} / \mathrm{cm}^{2}\right) \\
(\mathrm{E}>0.18 \mathrm{MeV}) \\
\text { HTGR }\end{array}$ & $\begin{array}{l}\text { Irrad } \\
\text { Temp } \\
\left({ }^{\circ} \mathrm{C}\right)\end{array}$ & $\begin{array}{c}\text { Irrad } \\
\text { Strain } \\
\ln (\ell / \ell o) \\
\left(10^{-2}\right)\end{array}$ & $\begin{array}{c}\text { Dimensional } \\
\text { Change, } \\
\Delta \ell / \ell_{0} \\
(\%)\end{array}$ \\
\hline $\begin{array}{l}5540 \\
5542 \\
5563 \\
5565 \\
5571 \\
5573 \\
5614 \\
5616 \\
5618 \\
5620 \\
5653 \\
5654 \\
5655 \\
5665 \\
5666 \\
5669 \\
5670 \\
5947 \\
5948 \\
5949 \\
5950 \\
5951 \\
5952 \\
5953 \\
6003 \\
6004 \\
6005 \\
6010 \\
6011 \\
6012 \\
6013 \\
6269 \\
6271 \\
6273 \\
6275\end{array}$ & $\begin{array}{l}2402 \\
2403 \\
2764 \\
2761 \\
2426 \\
2419 \\
2791 \\
2816 \\
2815 \\
2821 \\
2882 \\
2866 \\
2871 \\
2872 \\
2870 \\
2853 \\
2850 \\
2605 \\
2612 \\
2594 \\
2606 \\
2537 \\
2649 \\
2642 \\
2671 \\
2653 \\
2673 \\
2659 \\
2680 \\
2648 \\
2667 \\
2522 \\
2448 \\
2500 \\
2452\end{array}$ & 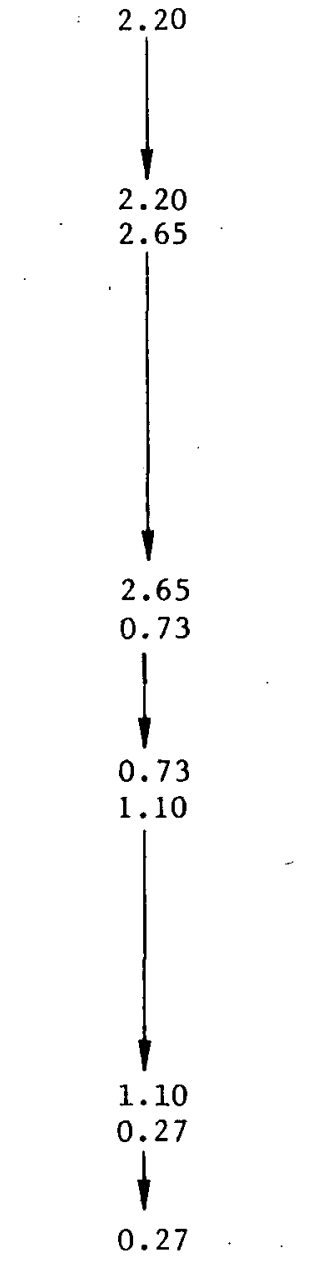 & $\begin{array}{c}1275 \\
1275 \\
1325 \\
1325 \\
1275 \\
1275 \\
1500 \\
\mid \\
1 \\
1500 \\
1475 \\
900 \\
1 \\
1000 \\
1 \\
1 \\
1 \\
625 \\
1\end{array}$ & 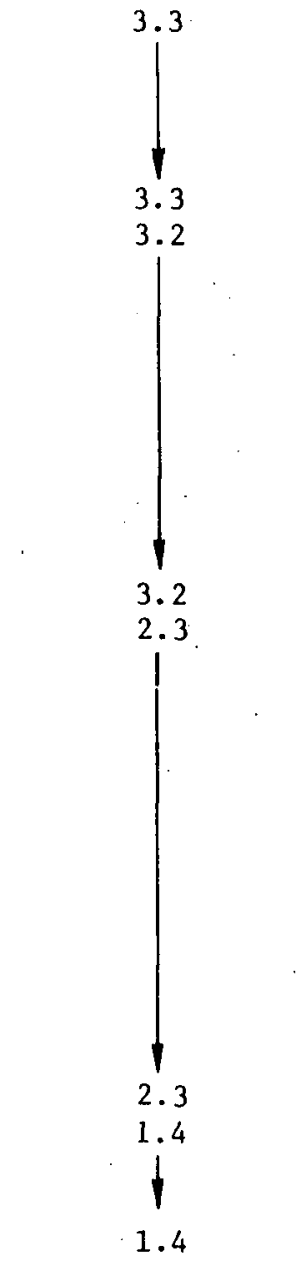 & 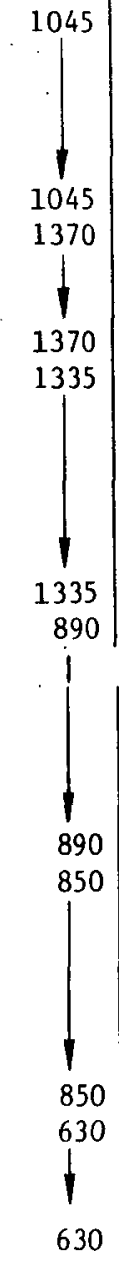 & 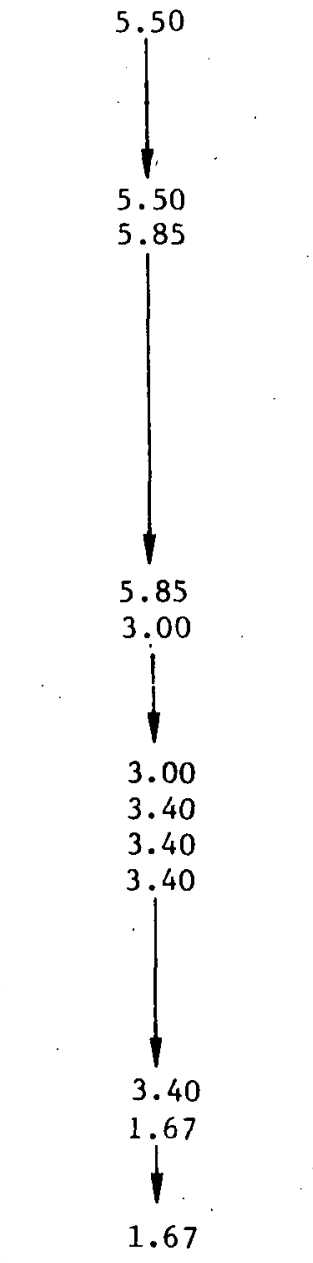 & $\begin{array}{l}1135 \\
1135 \\
1155 \\
1155 \\
1135 \\
1135 \\
1430 \\
1 \\
1430 \\
1410 \\
1410 \\
1400 \\
890 \\
\mid \\
8 \\
9 \\
925 \\
925 \\
900 \\
630 \\
6\end{array}$ & $\begin{array}{l}-1.86 \\
-1.50 \\
-2.14 \\
-1.45 \\
-2.10 \\
-1.71 \\
-3.12 \\
-2.83 \\
-3.74 \\
-3.52 \\
-3.43 \\
-2.84 \\
-3.29 \\
-3.09 \\
-3.40 \\
-3.15 \\
-2.95 \\
-0.54 \\
-0.48 \\
-0.36 \\
-0.61 \\
-0.53 \\
-0.63 \\
-0\end{array}$ & $\begin{array}{l}-1.84 \\
-1.50 \\
-2.12 \\
-1.44 \\
-2.08 \\
-1.69 \\
-3.07 \\
-2.79 \\
-3.67 \\
-3.46 \\
-3.37 \\
-2.80 \\
-3.23 \\
-3.04 \\
-3.34 \\
-3.10 \\
-2.95\end{array}$ \\
\hline
\end{tabular}


- TABLE 5-3

DIMENSIONAL CHANGE DATA, H-429, AXIAL DIRECTION, AFTER IRRADIATION IN CAPSULE OG-2

\begin{tabular}{|c|c|c|c|c|c|c|c|c|c|}
\hline $\begin{array}{c}\text { Record } \\
\text { No. }\end{array}$ & $\begin{array}{c}\text { Previous } \\
\text { Record } \\
\text { No. }\end{array}$ & $\begin{array}{c}\text { Previous } \\
\text { Fluence } \\
\left(10^{21} \mathrm{n} / \mathrm{cm}^{2}\right) \\
(\mathrm{E}>0.18 \mathrm{MeV})_{\text {HTGR }}\end{array}$ & $\begin{array}{c}\text { Previous } \\
\text { Temp } \\
\left({ }^{\circ} \mathrm{C}\right)\end{array}$ & $\begin{array}{c}\text { Fluence } \\
\text { This Irrad } \\
\left(10^{21} \mathrm{n} / \mathrm{cm}^{2}\right) \\
(\mathrm{E}>0.18 \mathrm{MeV}) \\
\end{array}$ & $\begin{array}{l}\text { Temp } \\
\text { This } \\
\text { Irrad } \\
\left({ }^{\circ} \mathrm{C}\right)\end{array}$ & $\begin{array}{c}\text { Total } \\
\text { Fluence } \\
\left(10^{21} \mathrm{n} / \mathrm{cm}^{2}\right) \\
(\mathrm{E}>0.18 \mathrm{MeV})_{\mathrm{HTGR}}\end{array}$ & $\begin{array}{l}\text { Irrad } \\
\text { Temp } \\
\left({ }^{\circ} \mathrm{C}\right)\end{array}$ & $\begin{array}{c}\text { Irrad } \\
\text { Strain } \\
\ln (\ell / \ell o) \\
\left(10^{-2}\right)\end{array}$ & $\begin{array}{c}\text { Dimensional } \\
\text { Change, } \\
\Delta \ell / \ell_{0} \\
(\%)\end{array}$ \\
\hline $\begin{array}{l}6924 \\
6926 \\
6928 \\
6930 \\
6932 \\
6934 \\
6990 \\
6992 \\
6994 \\
6996 \\
6997 \\
6998 \\
6999 \\
7005 \\
7006 \\
7009 \\
7010 \\
7354 \\
7355 \\
7356 \\
7357 \\
7358 \\
7359 \\
7360 \\
7391 \\
7392 \\
7393 \\
7394 \\
7395 \\
7396 \\
7397 \\
7557 \\
7559 \\
7561 \\
7563\end{array}$ & $\begin{array}{l}5540 \\
5542 \\
5563 \\
5565 \\
5571 \\
5573 \\
5614 \\
5616 \\
5618 \\
5620 \\
5653 \\
5654 \\
5655 \\
5665 \\
5666 \\
5669 \\
5670 \\
5947 \\
5948 \\
5949 . \\
5950 \\
5951 \\
5952 \\
5953 \\
6003 \\
6004 \\
6005 \\
6010 \\
6011 \\
6012 \\
6013 \\
6269 \\
6271 \\
6273 \\
6275\end{array}$ & $\begin{array}{c}\mid \\
5.85 \\
3.00 \\
\left.\right|_{3.00} \\
3.40 \\
\left.\right|^{3.40}\end{array}$ & $\begin{array}{c}1135 \\
1135 \\
1155 \\
1155 \\
1135 \\
1135 \\
1430 \\
\mid \\
1430 \\
1410 \\
\mid \\
1410 \\
1400 \\
1400 \\
890 \\
\mid \\
890 \\
925 \\
925 \\
900 \\
\mid\end{array}$ & 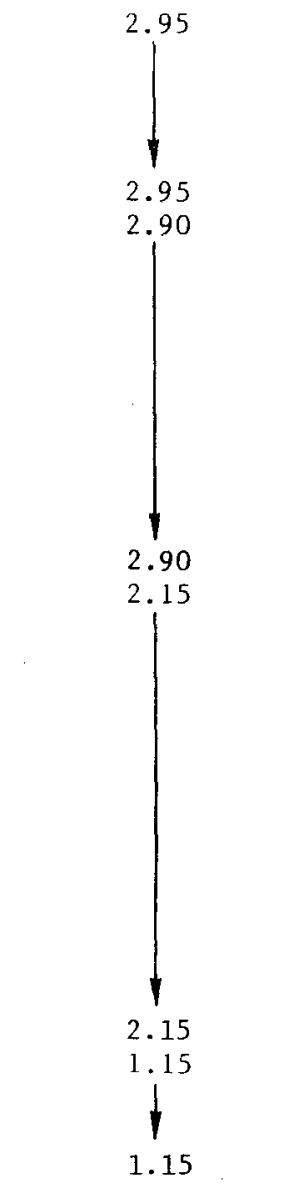 & 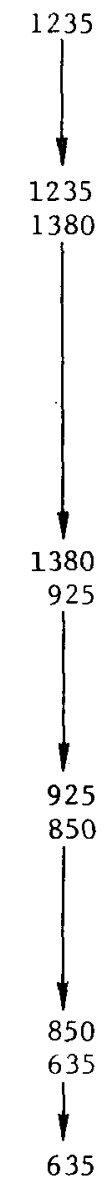 & $\begin{array}{l}\left.\right|_{1} . \\
8.75 \\
5.15 \\
\left.\right|_{5.15} \\
5.55\end{array}$ & $\begin{array}{r}1170 \\
1170 \\
1185 \\
1185 \\
1170 \\
1170 \\
1415 \\
1415 \\
1415 \\
1415 \\
1400 \\
1400 \\
1400 \\
1400 \\
1400 \\
1395 \\
1395 \\
905 \\
905 \\
905 \\
905 \\
905 \\
925 \\
925 \\
880 \\
880 \\
880 \\
880 \\
880 \\
880 \\
880 \\
630 \\
630 \\
630 \\
630\end{array}$ & $\begin{array}{l}-2.48 \\
-2.26 \\
-2.75 \\
-2.80 \\
-2.72 \\
-2.74 \\
-3.69 \\
-3.78 \\
-3.73 \\
-3.71 \\
-3.88 \\
-3.74 \\
-3.77 \\
-3.61 \\
-3.95 \\
-3.22 \\
-3.60 \\
-1.40 \\
-1.32 \\
-1.25 \\
-1.60 \\
-1.34 \\
-1.34 \\
-1.24 \\
-1.51 \\
-1.30 \\
-1.42 \\
-1.36 \\
-1.39 \\
-1.29 \\
-1.28 \\
-0.48 \\
-0.34 \\
-0.51 \\
-0.30\end{array}$ & $\begin{array}{l}-2.45 \\
-2.19 \\
-2.72 \\
-2.76 \\
-2.69 \\
-2.71 \\
-3.62 \\
-3.63 \\
-3.67 \\
-3.64 \\
-3.81 \\
-3.67 \\
-3.70 \\
-3.55 \\
-3.88 \\
-3.17 \\
-3.53 \\
-1.39 \\
-1.31 \\
-1.24 \\
-1.59 \\
-1.33 \\
-1.33 \\
-1.23 \\
-1.50 \\
-1.29 \\
-1.41 \\
-1.35 \\
-1.38 \\
-1.28 \\
-1.27\end{array}$ \\
\hline
\end{tabular}


TABLE 5-4

DIMENSIONAL CHANGE DATA, H-451, AXIAL DIRECTION, AFTER IRRADIATION IN CAPSULE OG-1

\begin{tabular}{|c|c|c|c|c|}
\hline $\begin{array}{c}\text { Record } \\
\text { No. }\end{array}$ & $\begin{array}{c}\text { Total } \\
\text { F1uence } \\
\left(10^{21} \mathrm{n} / \mathrm{cm}^{2}\right) \\
(\mathrm{E}>0.18 \mathrm{MeV}) \\
{ }_{\text {HTGR }}\end{array}$ & $\begin{array}{l}\text { Irrad } \\
\text { Temp } \\
\left({ }^{\circ} \mathrm{C}\right)\end{array}$ & $\begin{array}{l}\text { Irrad } \\
\text { Strain } \\
\ln \left(\ell / \ell_{0}\right) \\
\left(10^{-2}\right)\end{array}$ & $\begin{array}{c}\text { Dimensional } \\
\text { Change, } \\
\Delta \ell / \ell_{0} \\
(\%)\end{array}$ \\
\hline 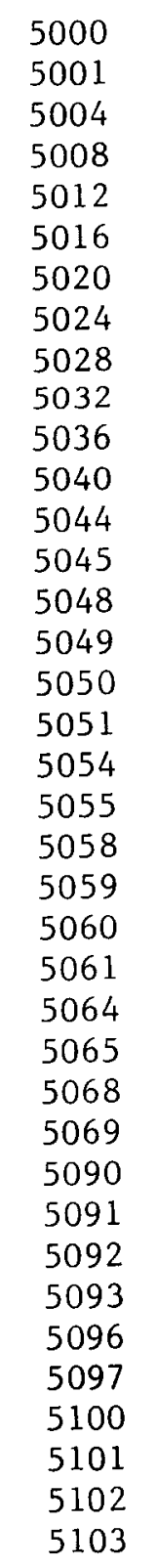 & $\left.\right|^{2.0}$ & $\left.\right|^{660}$ & $\begin{array}{l}-0.05 \\
-0.10 \\
-0.12 \\
-0.11 \\
-0.10 \\
-0.10 \\
-0.14 \\
-0.09 \\
-0.05 \\
-0.12 \\
-0.24 \\
-0.19 \\
-0.11 \\
-0.13 \\
-0.12 \\
-0.15 \\
-0.12 \\
-0.11 \\
-0.10 \\
-0.14 \\
-0\end{array}$ & \\
\hline
\end{tabular}


TABLE 5-4 (continued)

\begin{tabular}{|c|c|c|c|c|}
\hline $\begin{array}{c}\text { Record } \\
\text { No. }\end{array}$ & $\begin{array}{c}\text { Tota1 } \\
\text { Fluence } \\
\left(1021 \mathrm{n} / \mathrm{cm}^{2}\right) \\
(\mathrm{E}>0.18 \mathrm{MeV})_{\text {HTGR }}\end{array}$ & $\begin{array}{c}\text { Irrad } \\
\text { Temp } \\
\left({ }^{\circ} \mathrm{C}\right)\end{array}$ & $\begin{array}{l}\text { Irrad } \\
\text { Strain } \\
\ln \left(\ell / \ell_{0}\right) \\
\left(10^{-2}\right)\end{array}$ & $\begin{array}{c}\text { Dimensional } \\
\text { Change, } \\
\Delta \ell / \ell_{0} \\
(\%)\end{array}$ \\
\hline $\begin{array}{l}5104 \\
5105 \\
5108 \\
5109 \\
5112 \\
5113 \\
5122 \\
5123 \\
5132 \\
5133 \\
5142 \\
5143 \\
5146 \\
5150 \\
5154 \\
5158 \\
5162 \\
5166 \\
5170 \\
5174 \\
5178 \\
6472 \\
5187 \\
5188 \\
5191 \\
5192 \\
5197 \\
5198 \\
5201 \\
5202 \\
5207 \\
5208 \\
5211 \\
5212 \\
5243 \\
5244 \\
5247 \\
5248 \\
5253 \\
5254\end{array}$ & $\left.\right|^{2.0}$ & $\mid$ & $\begin{array}{l}-0.16 \\
-0.19 \\
-0.16 \\
-0.15 \\
-0.16 \\
-0.22 \\
-0.18 \\
-0.14 \\
-0.15 \\
-0.35 \\
-0.14 \\
-0.23 \\
-0.12 \\
-0.15 \\
-0.16 \\
-0.22 \\
-0.28 \\
-0.17 \\
-0.13 \\
-0.12 \\
-0.17 \\
-0.24 \\
-0.16 \\
-0.23 \\
-0.10 \\
-0.12 \\
-0.19 \\
-0.27 \\
-0.21 \\
-0.02 \\
-0.22 \\
-0.24 \\
-0.26 \\
-0.30 \\
-0.15 \\
-0.17 \\
-0.24 \\
-0.23 \\
-0.25 \\
-0.28\end{array}$ & \\
\hline
\end{tabular}


TABLE 5-4 (continued)

\begin{tabular}{|c|c|c|c|}
\hline $\begin{array}{c}\text { Total } \\
\text { Fluence } \\
\left(10^{21} \mathrm{n} / \mathrm{cm}^{2}\right) \\
(\mathrm{E}>0.18 \mathrm{MeV})_{\text {HTGR }}\end{array}$ & $\begin{array}{l}\text { Irrad } \\
\text { Temp } \\
\left({ }^{\circ} \mathrm{C}\right)\end{array}$ & $\begin{array}{c}\text { Irrad } \\
\text { Strain } \\
\ln (\ell / \ell 0) \\
\left(10^{-2}\right)\end{array}$ & $\begin{array}{c}\text { Dimensional } \\
\text { Change, } \\
\Delta \ell / \ell_{0} \\
(\%)\end{array}$ \\
\hline X & 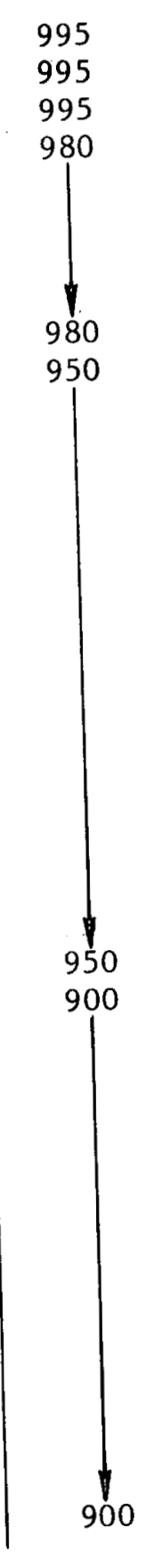 & $\begin{array}{l}-0.72 \\
-0.78 \\
-1.06 \\
-0.64 \\
-0.90 \\
-0.77 \\
-0.61 \\
-0.79 \\
-0.99 \\
-0.68 \\
-0.86 \\
-0.61 \\
-0.86 \\
-0.56 \\
-0.74 \\
-0.78 \\
-0.59 \\
-0.77 \\
-0.59 \\
-0.82 \\
-0.51 \\
-0.60 \\
-0.80 \\
-0.83 \\
-0.67 \\
-0.85 \\
-0.50 \\
-0.63 \\
-0.50 \\
-0.57 \\
-0.57 \\
-0.48 \\
-0.60 \\
-0.62 \\
-0.51 \\
-0.65 \\
-0.48 \\
-0.54 \\
-0.54 \\
-0.47 \\
-0.58\end{array}$ & -1.05 \\
\hline
\end{tabular}


TABLE 5-4 (continued)

\begin{tabular}{|c|c|c|c|c|}
\hline $\begin{array}{c}\text { Record } \\
\text { No. }\end{array}$ & $\begin{array}{c}\text { Total } \\
\text { Fluence } \\
\left(10^{21} \mathrm{n} / \mathrm{cm}^{2}\right) \\
(\mathrm{E}>0.18 \mathrm{MeV})_{\text {HTGR }} \\
\end{array}$ & $\begin{array}{c}\text { Irrad } \\
\text { Temp } \\
\left({ }^{\circ} \mathrm{C}\right)\end{array}$ & $\begin{array}{c}\text { Irrad } \\
\text { Strain } \\
\ln (\ell / \ell) \\
\left(10^{-2}\right)\end{array}$ & $\begin{array}{c}\text { Dimensional } \\
\text { Change, } \\
\Delta \ell / \ell_{0} \\
(\%)\end{array}$ \\
\hline 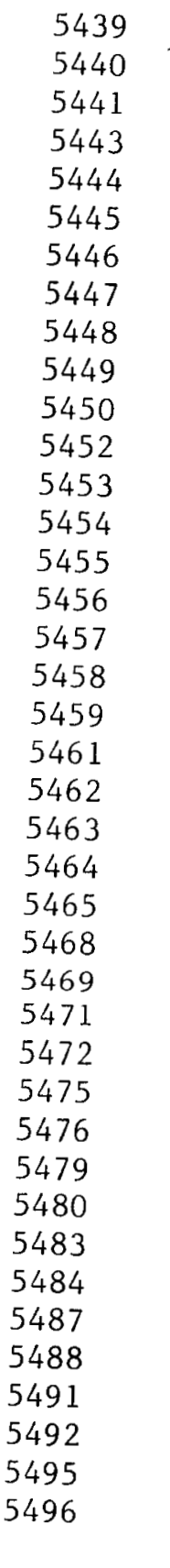 & $\left.\right|^{3.0}$ & \begin{tabular}{|}
1180 \\
1160 \\
900 \\
1160
\end{tabular} & $\begin{array}{l}-0.58 \\
-0.43 \\
-0.58 \\
-0.41 \\
-0.50 \\
-0.42 \\
-0.37 \\
-0.52 \\
-0.43 \\
-0.41 \\
-0.55 \\
-0.42 \\
-0.45 \\
-0.50 \\
-0.39 \\
-0.51 \\
-0.54 \\
-0.42 \\
-0.54 \\
-0.41 \\
-0.49 \\
-0.56 \\
-0.47 \\
-0.51 \\
-0.46 \\
-0.57 \\
-0.47 \\
-0.55 \\
-0.68 \\
-0.41 \\
-1.09 \\
-1.20 \\
-1.07 \\
-1.14 \\
-1.02 \\
-1.13 \\
-1.07 \\
-1.11 \\
-1.04 \\
-0.99\end{array}$ & $\begin{array}{l}-1.08 \\
-1.20 \\
-1.06 \\
-1.13 \\
-1.01 \\
-1.13 \\
-1.06 \\
-1.10 \\
-1.03 \\
-0.98\end{array}$ \\
\hline
\end{tabular}


TABLE 5-4 (continued)

\begin{tabular}{|c|c|c|c|c|}
\hline $\begin{array}{c}\text { Record } \\
\text { No. }\end{array}$ & $\begin{array}{c}\text { Total } \\
\text { F1uence } \\
\left(10^{21} \mathrm{n} / \mathrm{cm}^{2}\right) \\
(\mathrm{E}>0.18 \mathrm{MeV}) \\
\end{array}$ & $\begin{array}{r}\text { Irrad } \\
\text { Temp } \\
\left({ }^{\circ} \mathrm{C}\right)\end{array}$ & $\begin{array}{l}\text { Irrad } \\
\text { Strain } \\
\ln (\ell / \ell o) \\
\left(10^{-2}\right)\end{array}$ & $\begin{array}{c}\text { Dimensional } \\
\text { Change, } \\
\Delta \ell / \ell_{0} \\
(\%)\end{array}$ \\
\hline 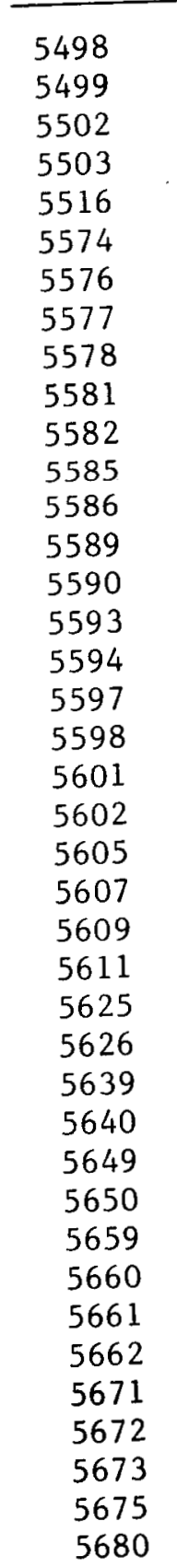 & \begin{tabular}{|}
3.3 \\
3.2 \\
3.2 \\
3.2 \\
$i^{3}$
\end{tabular} & \begin{tabular}{|}
1160 \\
1115 \\
1045 \\
1045 \\
1395 \\
1395 \\
1385 \\
$\mid$ \\
1385 \\
1370 \\
1335 \\
$\mid$
\end{tabular} & $\begin{array}{l}-1.01 \\
-1.17 \\
-0.97 \\
-1.08 \\
-1.27 \\
-1.22 \\
-1.19 \\
-3.00 \\
-2.74 \\
-2.85 \\
-2.99 \\
-3.06 \\
-3.05 \\
-2.92 \\
-3.07 \\
-3.01 \\
-3.05 \\
-2.88 \\
-3.02 \\
-3.07 \\
-3.00 \\
-2.76 \\
-2.81 \\
-2.77 \\
-3.09 \\
-2.88 \\
-2.63 \\
-2.90 \\
-2.71 \\
-2.61 \\
-2.43 \\
-2.43 \\
-2.45 \\
-2.58 \\
-2.49 \\
-2.50 \\
-2.21 \\
-2.35 \\
-1.80 \\
-2.59\end{array}$ & $\begin{array}{l}-1.00 \\
-1.17 \\
-0.97 \\
-1.07 \\
-1.26 \\
-1.21 \\
-1.19 \\
-2.96 \\
-2.70 \\
-2.81 \\
-2.94 \\
-3.01 \\
-3.00 \\
-2.87 \\
-3.03 \\
-2.97 \\
-3.01 \\
-2.84 \\
-2.98 \\
-3.03 \\
-2.96 \\
-2.73 \\
-2.77 \\
-2.74 \\
-3.05 \\
-2.84 \\
-2.60 \\
-2.86 \\
-2.68 \\
-2.58 \\
-2.40 \\
-2.40 \\
-2.42 \\
-2.54 \\
-2.46 \\
-2.47 \\
-2.19 \\
-2.32 \\
-1.78 \\
-2.55 \\
\end{array}$ \\
\hline
\end{tabular}


TABLE 5-4 (continued)

\begin{tabular}{|c|c|c|c|c|}
\hline $\begin{array}{c}\text { Record } \\
\text { No. }\end{array}$ & $\begin{array}{c}\text { Tota1 } \\
\text { Fluence } \\
\left(10^{21} \mathrm{n} / \mathrm{cm}^{2}\right) \\
(E>0.18 \mathrm{MeV}) \\
\end{array}$ & $\begin{array}{l}\text { Irrad } \\
\text { Temp } \\
\left({ }^{\circ} \mathrm{C}\right)\end{array}$ & $\begin{array}{c}\text { Irrad } \\
\text { Strain } \\
\ln (\ell / \ell o) \\
(10-2) \\
\end{array}$ & $\begin{array}{c}\text { Dimensional } \\
\text { Change, } \\
\Delta \ell / \ell_{0} \\
(\%)\end{array}$ \\
\hline $\begin{array}{l}5681 \\
5682 \\
5683 \\
5688 \\
5690 \\
5692 \\
5693 \\
5696 \\
5697 \\
5700 \\
5701 \\
5704 \\
5705 \\
5710 \\
5711 \\
5714 \\
5715 \\
5718 \\
5719 \\
5722 \\
5723 \\
5726 \\
5727 \\
5730 \\
5731 \\
5764 \\
5773 \\
5777 \\
5781 \\
5782 \\
5786 \\
5788 \\
5789 \\
5793 \\
5795 \\
5799 \\
5801 \\
5803 \\
5810 \\
5814\end{array}$ & $\left.\right|_{3.2} ^{3.2}$ & \begin{tabular}{|}
1335 \\
1085 \\
1085 \\
1065 \\
$\mid$ \\
1065 \\
1025 \\
1025 \\
1025 \\
960 \\
980 \\
980 \\
$\mid$
\end{tabular} & $\begin{array}{l}-2.25 \\
-2.61 \\
-2.30 \\
-2.48 \\
-2.39 \\
-1.26 \\
-1.23 \\
-1.16 \\
-1.11 \\
-1.15 \\
-1.08 \\
-1.17 \\
-1.05 \\
-1.14 \\
-1.09 \\
-1.13 \\
-1.10 \\
-1.13 \\
-1.08 \\
-1.17 \\
-1.06 \\
-1.12 \\
-1.03 \\
-1.10 \\
-0.97 \\
-0.63 \\
-0.57 \\
-0.54 \\
-0.50 \\
-0.68 \\
-0.61 \\
-0.50 \\
-0.67 \\
-0.62 \\
-0.70 \\
-0.72 \\
-0.66 \\
-0.81 \\
-0.69 \\
-0.73\end{array}$ & $\begin{array}{l}-2.23 \\
-2.58 \\
-2.28 \\
-2.45 \\
-2.36 \\
-1.25 \\
-1.22 \\
-1.15 \\
-1.11 \\
-1.15 \\
-1.07 \\
-1.16 \\
-1.04 \\
-1.14 \\
-1.09 \\
-1.12 \\
-1.09 \\
-1.12 \\
-1.07 \\
-1.16 \\
-1.05 \\
-1.12 \\
-1.02 \\
-1.09 \\
-0.96 \\
-0.62\end{array}$ \\
\hline
\end{tabular}


TABLE 5-4 (continued)

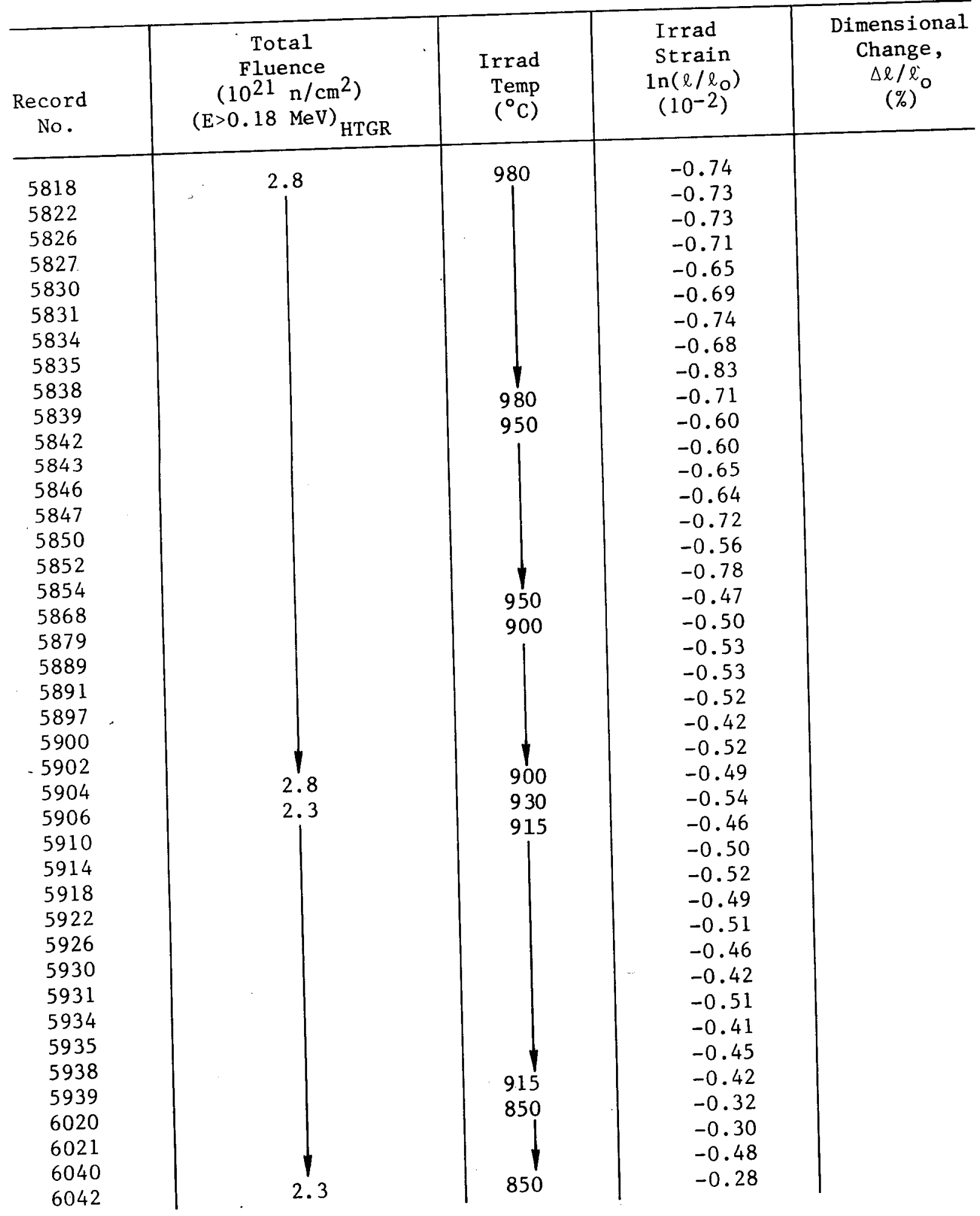


TABLE 5-4 (continued)

\begin{tabular}{|c|c|c|c|c|}
\hline $\begin{array}{c}\text { Record } \\
\text { No. }\end{array}$ & $\begin{array}{c}\text { Total } \\
\text { Fluence } \\
\left(10^{21} \mathrm{n} / \mathrm{cm}^{2}\right) \\
(\mathrm{E}>0.18 \mathrm{MeV})_{\mathrm{HTGR}}\end{array}$ & $\begin{array}{c}\text { Irrad } \\
\text { Temp } \\
\left({ }^{\circ} \mathrm{C}\right)\end{array}$ & $\begin{array}{c}\text { Irrad } \\
\text { Strain } \\
\ln \left(\ell / \ell_{0}\right) \\
\left(10^{-2}\right)\end{array}$ & $\begin{array}{c}\text { Dimensional } \\
\text { Change, } \\
\Delta \ell / \ell_{0} \\
(\%)\end{array}$ \\
\hline 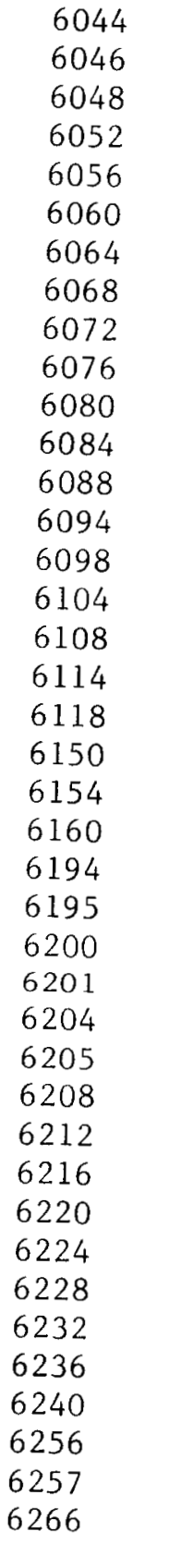 & $\left.\right|^{2.3}$ & 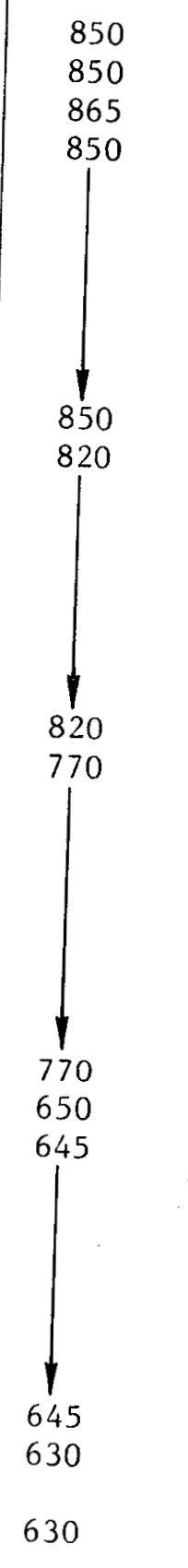 & $\begin{array}{r}-0.32 \\
-0.25 \\
-0.21 \\
-0.18 \\
-0.18 \\
-0.18 \\
-0.17 \\
-0.21 \\
-0.18 \\
-0.16 \\
-0.22 \\
-0.34 \\
-0.10 \\
-0.22 \\
-0.13 \\
-0.16 \\
-0.15 \\
-0.14 \\
-0.16 \\
-0.15 \\
-0.13 \\
-0.14 \\
-0.13 \\
-0.14 \\
-0.12 \\
-0.23 \\
-0.14 \\
-0.16 \\
-0.06 \\
-0.04 \\
0.00 \\
-0.07 \\
-0.10 \\
-0.07 \\
-0.10 \\
-0.22 \\
-0.08 \\
-0.16 \\
-0.13 \\
-0.12\end{array}$ & \\
\hline
\end{tabular}


TABLE 5-4 (continued)

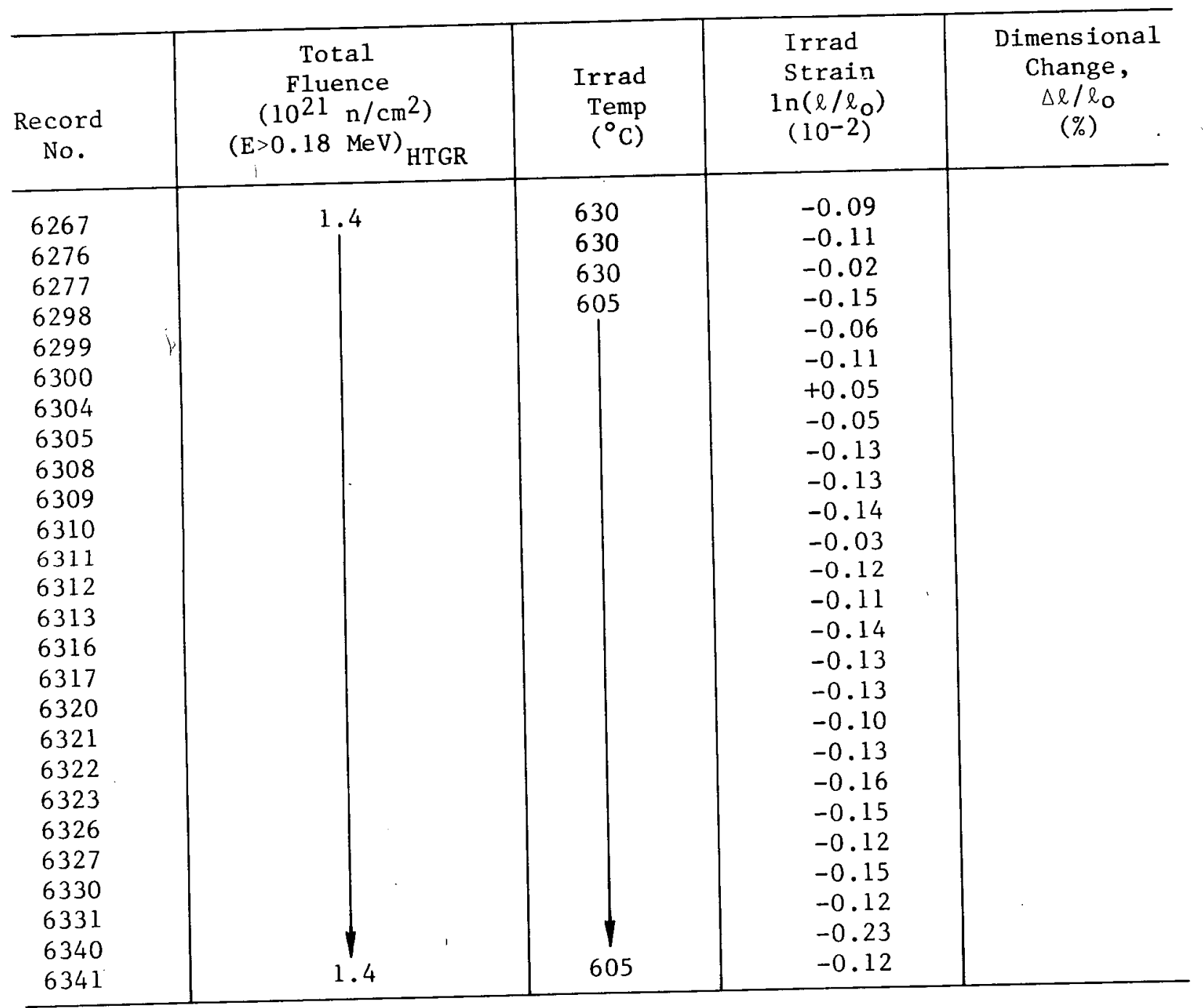


TABLE 5-5

DIMENSIONAL CHANGE DATA, H-451, AXIAL DIRECTION, AFTER IRRADIATION IN CAPSULE OG-2

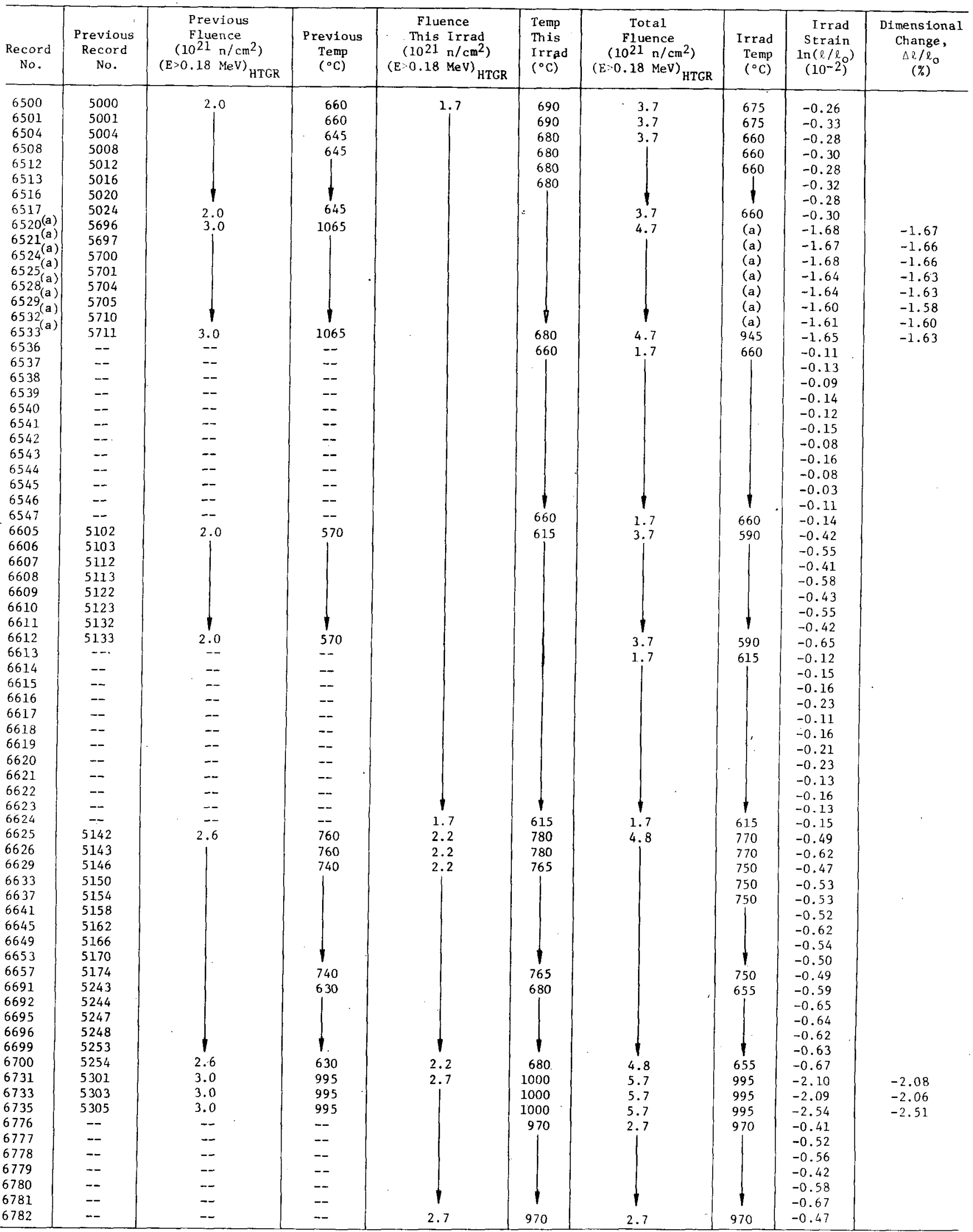




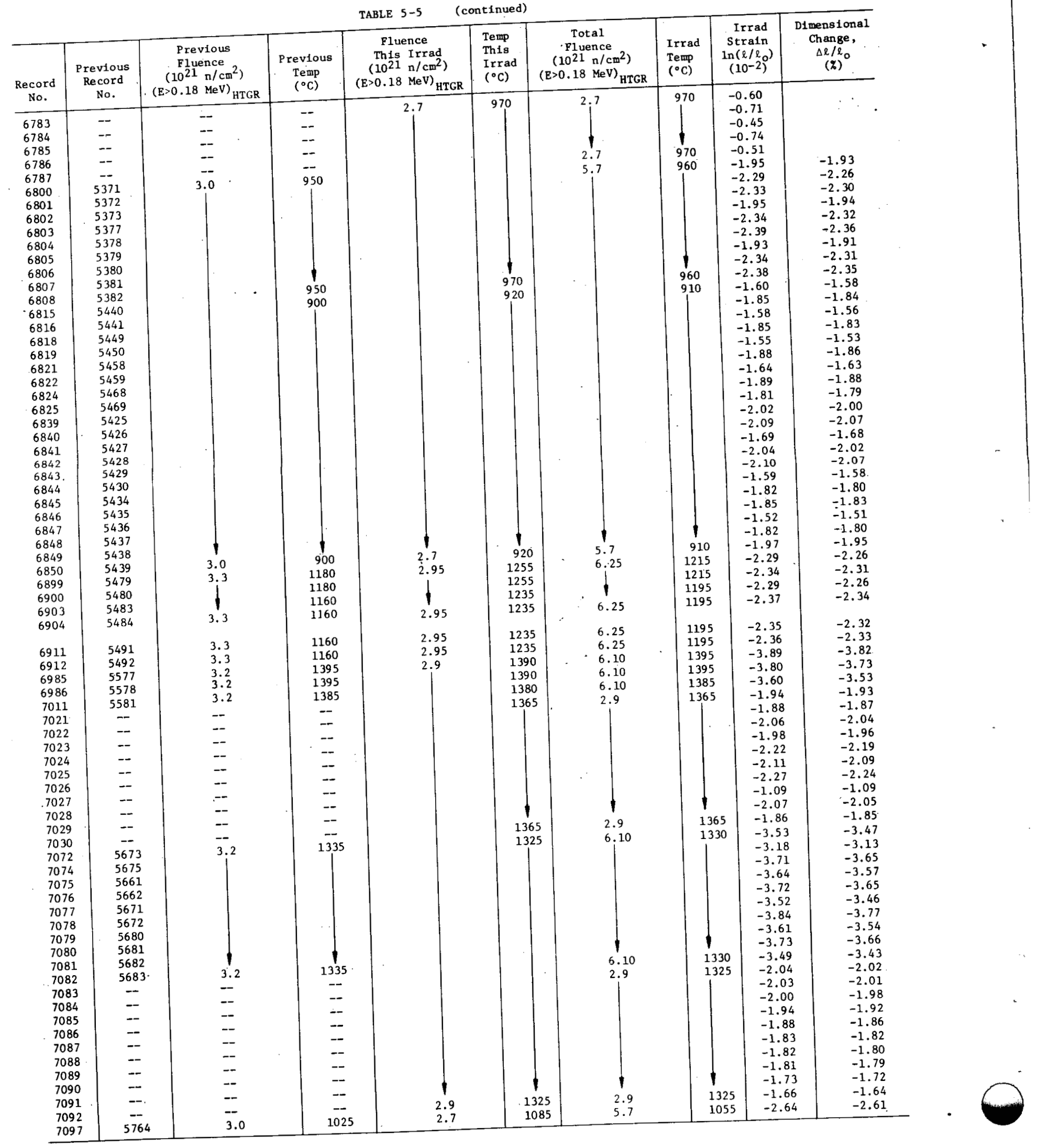


TABLE 5-5 (continued)

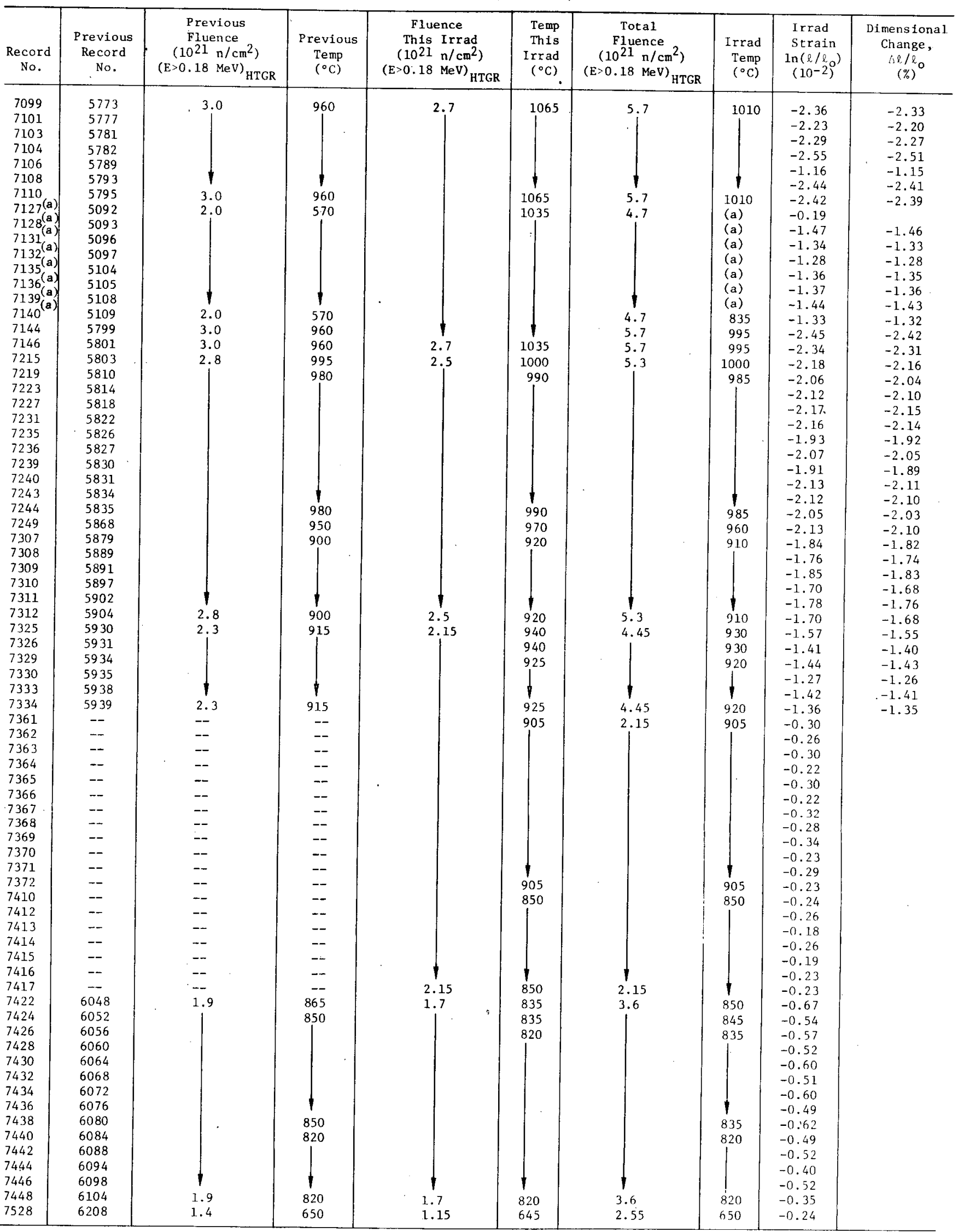




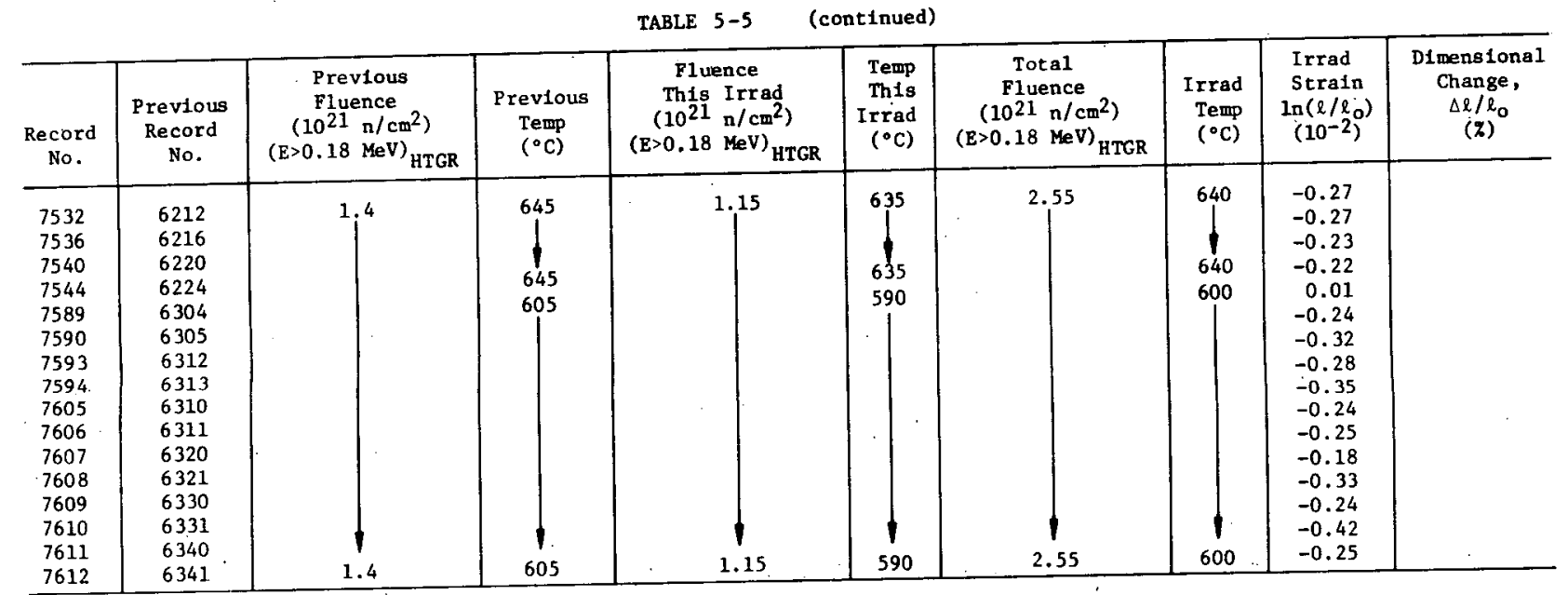

(a) Temperature change samples. 
TABLE 5-6

DIMENSIONAL CHANGE DATA, H-429, RADIAL DIRECTION, AFTER IRRADIATION IN CAPSULES GEH-422, 452, 482

\begin{tabular}{|c|c|c|c|}
\hline $\begin{array}{c}\text { Record } \\
\text { No. }\end{array}$ & $\begin{array}{c}\text { Total } \\
\text { Fluence } \\
\left(1021 \mathrm{n} / \mathrm{cm}^{2}\right) \\
(\mathrm{E}>0.18 \mathrm{MeV})_{\text {HTGR }}\end{array}$ & $\begin{array}{r}\text { Irrad } \\
\text { Temp } \\
\left({ }^{\circ} \mathrm{C}\right)\end{array}$ & $\begin{array}{l}\text { Irrad } \\
\text { Strain } \\
\ln \left(\ell / \ell_{0}\right) \\
\left(10^{-2}\right)\end{array}$ \\
\hline 3609 & 3.35 & 475 & -0.60 \\
\hline 3610 & 3.40 & 475 & -0.59 \\
\hline 3611 & 3.45 & 475 & -0.60 \\
\hline 3625 & 2.80 & 525 & -0.59 \\
\hline 3606 & 3.20 & 525 & -0.62 \\
\hline 3607 & 3.25 & 525 & -0.65 \\
\hline 3608 & 3.30 & 525 & -0.61 \\
\hline 2461 & 0.27 & 625 & 0.00 \\
\hline 2467 & & & -0.01 \\
\hline 2488 & & & +0.02 \\
\hline 2497 & & & +0.01 \\
\hline 2518 & & & +0.02 \\
\hline 2521 & & & +0.00 \\
\hline 2524 & & 1 & +0.02 \\
\hline 2532 & 0.27 & 625 & +0.00 \\
\hline 3725 & 2.80 & 775 & -0.34 \\
\hline 3706 & 3.20 & 775 & -0.43 \\
\hline 3707 & 3.25 & 775 & -0.47 \\
\hline 3708 & 3.30 & 775 & -0.52 \\
\hline 3709 & 3.35 & 800 & -0.50 \\
\hline 3710 & 3.40 & 800 & -0.45 \\
\hline 3711 & 3.45 & 800 & -0.53 \\
\hline 2564 & 0.73 & 900 & +0.12 \\
\hline 2580 & & & +0.01 \\
\hline 2598 & & & +0.03 \\
\hline 2601 & & & +0.01 \\
\hline 2608 & 0.73 & 900 & +0.03 \\
\hline 2645 & 1.10 & 1000 & -0.20 \\
\hline 2652 & & 1 & -0.10 \\
\hline 2655 & & & -0.10 \\
\hline 2662 & & & -0.10 \\
\hline 2663 & & & -0.06 \\
\hline 2665 & & & -0.05 \\
\hline 2672 & & & -0.14 \\
\hline 2681 & & 1 & -0.08 \\
\hline 2687 & 1.10 & 1000 & -0.03 \\
\hline 2688 & 1.50 & 1100 & -0.06 \\
\hline 2690 & & & -0.08 \\
\hline 2691 & & & -0.14 \\
\hline 2702 & 1 & 1 & -0.14 \\
\hline 2712 & 1.50 & 1100 & -0.12 \\
\hline
\end{tabular}


TABLE 5-6 (continued)

\begin{tabular}{|c|c|c|c|}
\hline $\begin{array}{c}\text { Record } \\
\text { No. }\end{array}$ & $\begin{array}{c}\text { Total } \\
\text { F1uence } \\
\left(10^{21} \mathrm{n} / \mathrm{cm}^{2}\right) \\
(\mathrm{E}>0.18 \mathrm{MeV}) \text { HTGR }\end{array}$ & $\begin{array}{r}\text { Irrad } \\
\text { Temp } \\
\left({ }^{\circ} \mathrm{C}\right)\end{array}$ & $\begin{array}{l}\text { Irrad } \\
\text { Strain } \\
\ln \left(\ell / \ell_{0}\right) \\
\left(10^{-2}\right)\end{array}$ \\
\hline $\begin{array}{l}2428 \\
2430 \\
2436 \\
2442 \\
2748 \\
2855 \\
2861 \\
2800 \\
2804 \\
2807 \\
2823 \\
2849 \\
2858 \\
2864 \\
2875 \\
2877\end{array}$ & $\left.\right|_{2.20} ^{2.65}$ & \begin{tabular}{|}
1275 \\
1325 \\
1475 \\
1475 \\
1500 \\
$\mid$ \\
1500
\end{tabular} & $\begin{array}{l}-0.64 \\
-0.86 \\
-0.81 \\
-0.98 \\
-1.11 \\
-1.61 \\
-1.60 \\
-1.55 \\
-1.46 \\
-1.59 \\
-1.48 \\
-1.54 \\
-1.62 \\
-1.68 \\
-1.66 \\
-1.64 \\
\end{array}$ \\
\hline
\end{tabular}



TABLE 5-8

DIMENS IONAL CHANGE DATA, H-429, RADIAL DIRECTION, AFTER IRRADIATION IN CAPSULE OG-2

\begin{tabular}{|c|c|c|c|c|c|c|c|c|c|}
\hline $\begin{array}{c}\text { Record } \\
\text { No. }\end{array}$ & $\begin{array}{c}\text { Previous } \\
\text { Record } \\
\text { No. }\end{array}$ & $\begin{array}{c}\text { Previous } \\
\text { F1uence } \\
\left(10^{21} \mathrm{n} / \mathrm{cm}^{2}\right) \\
(E>0.18 \mathrm{MeV}){ }_{\text {HTGR }}\end{array}$ & $\begin{array}{l}\text { Previous } \\
\text { Temp } \\
\left({ }^{\circ} \mathrm{C}\right)\end{array}$ & $\begin{array}{c}\text { Fluence } \\
\text { This Irrad } \\
\left(10^{21} \mathrm{n} / \mathrm{cm}^{2}\right) \\
(\mathrm{E}>0.18 \mathrm{MeV})_{\text {HTGR }}\end{array}$ & $\begin{array}{l}\text { Temp } \\
\text { This } \\
\text { Irrad } \\
\left({ }^{\circ} \mathrm{C}\right)\end{array}$ & $\begin{array}{c}\text { Total } \\
\text { F1uence } \\
\left(10^{21} \mathrm{n} / \mathrm{cm}^{2}\right) \\
(\mathrm{E}>0.18 \mathrm{MeV})_{\text {HTGR }}\end{array}$ & $\begin{array}{r}\text { Irrad } \\
\text { Temp } \\
\left({ }^{\circ} \mathrm{C}\right)\end{array}$ & $\begin{array}{l}\text { Irrad } \\
\text { Strain } \\
\ln (\ell / \ell) \\
\left(10^{-2}\right)\end{array}$ & $\begin{array}{c}\text { Dimensional } \\
\text { Change, } \\
\Delta \ell / \ell_{0} \\
(\%)\end{array}$ \\
\hline 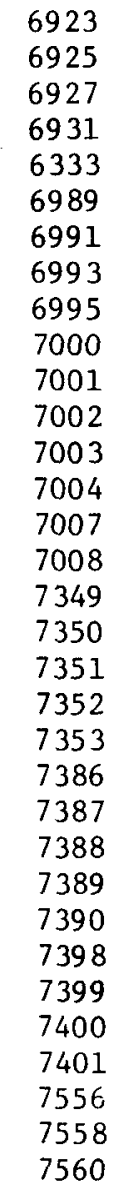 & $\begin{array}{l}5539 \\
5541 \\
5562 \\
5570 \\
5572 \\
5613 \\
5615 \\
5617 \\
5619 \\
5656 \\
5657 \\
5658 \\
5663 \\
5664 \\
5667 \\
5668 \\
5942 \\
5943 \\
5944 \\
5945 \\
5946 \\
5998 \\
5999 \\
6000 \\
6001 \\
6002 \\
6014 \\
6015 \\
6016 \\
6017 \\
6268 \\
6270 \\
6272\end{array}$ & \begin{tabular}{|}
5.5 \\
5.85 \\
3.0 \\
1 \\
3.0 \\
3.4 \\
1.67 \\
1.67 \\
1 \\
1.67
\end{tabular} & $\begin{array}{c}1135 \\
1135 \\
1155 \\
1135 \\
1135 \\
1430 \\
\mid \\
1430 \\
1410 \\
1410 \\
1400 \\
1400 \\
890 \\
\mid \\
890 \\
900 \\
630 \\
630\end{array}$ & \begin{tabular}{|}
2.95 \\
2.95 \\
2.9 \\
2.15 \\
1
\end{tabular} & $\mid$ & \begin{tabular}{|} 
|. \\
8.45 \\
8.75 \\
\\
8.75 \\
5.15 \\
$\mid$ \\
5.15 \\
5.55 \\
1 \\
2.85 \\
2.82 \\
\end{tabular} & $\begin{array}{l}1170 \\
1170 \\
1185 \\
1170 \\
1170 \\
1415 \\
\mid \\
1415 \\
1400 \\
1400 \\
1395 \\
1395 \\
905 \\
\mid \\
905 \\
880 \\
630 \\
630 \\
630\end{array}$ & $\begin{array}{l}-1.27 \\
-1.20 \\
-1.62 \\
-1.43 \\
-1.35 \\
-2.17 \\
-1.87 \\
-1.79 \\
-1.06 \\
-2.30 \\
-2.18 \\
-2.36 \\
-2.04 \\
-2.07 \\
-1.42 \\
-1.68 \\
-0.78 \\
-0.73 \\
-0.61 \\
-0.74 \\
-0.76 \\
-0.91 \\
-0.86 \\
-0.95 \\
-0.91 \\
-0.80 \\
-0.97 \\
-0.92 \\
-0.88 \\
-0.48 \\
-0.46 \\
-0.33 \\
-0.45\end{array}$ & $\begin{array}{l}-1.26 \\
-1.19 \\
-1.61 \\
-1.42 \\
-1.34 \\
-2.14 \\
-1.85 \\
-1.78 \\
-1.06 \\
-2.28 \\
-2.16 \\
-2.34 \\
-2.02 \\
-2.05 \\
-1.41 \\
-1.67\end{array}$ \\
\hline
\end{tabular}


TABLE 5-9

DIMENSIONAL CHANGE DATA, H-451, RADIAL DIRECTION, AFTER IRRADIATION IN CAPSULE OG- 1

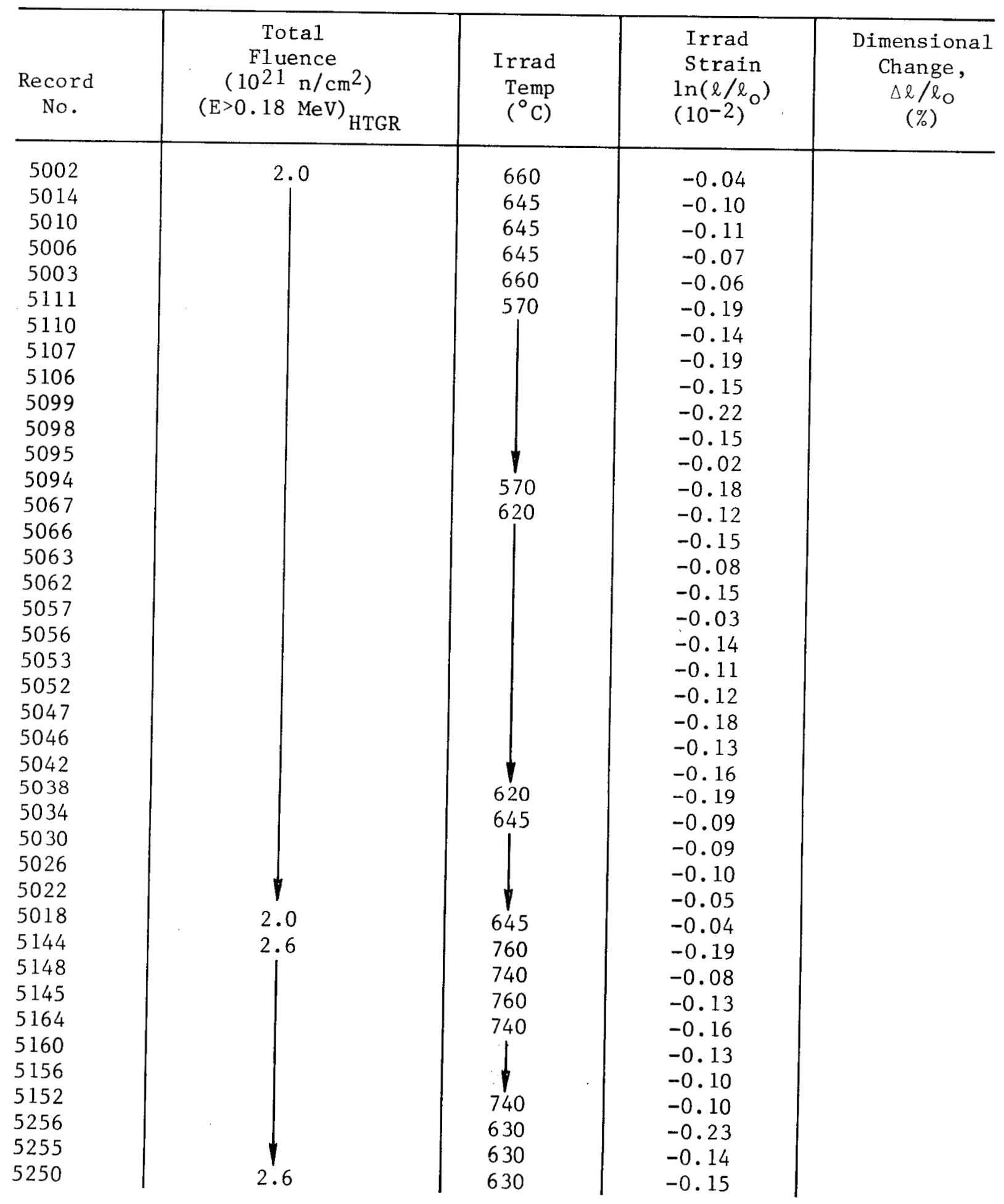


TABLE 5-9 (continued)

\begin{tabular}{|c|c|c|c|c|}
\hline $\begin{array}{c}\text { Record } \\
\text { No. }\end{array}$ & $\begin{array}{c}\text { Total } \\
\text { F1uence } \\
\left(10^{21} \mathrm{n} / \mathrm{cm}^{2}\right) \\
(\mathrm{E}>0.18 \mathrm{MeV}) \\
\text { HTGR }\end{array}$ & $\begin{array}{l}\text { Irrad } \\
\text { Temp } \\
\left({ }^{\circ} \mathrm{C}\right)\end{array}$ & $\begin{array}{l}\text { Irrad } \\
\text { Strain } \\
\ln \left(\ell / \ell_{0}\right) \\
\left(10^{-2}\right)\end{array}$ & $\begin{array}{c}\text { Dimensional } \\
\text { Change, } \\
\Delta \ell / \ell_{O} \\
(\%)\end{array}$ \\
\hline $\begin{array}{l}5249 \\
5246 \\
5245 \\
5214 \\
5213 \\
5210 \\
5209 \\
5204 \\
5203 \\
5200 \\
5199 \\
5194 \\
5193 \\
5190 \\
5189 \\
5183 \\
5180 \\
5176 \\
5172 \\
5168 \\
5302 \\
5318 \\
5316 \\
5314 \\
5312 \\
5310 \\
5308 \\
5306 \\
5304 \\
5433 \\
5424 \\
5385 \\
5376 \\
5368 \\
5367 \\
5365 \\
5361 \\
5360 \\
5355 \\
5355 \\
5354 \\
5351 \\
5350\end{array}$ & $j^{2.6}$ & 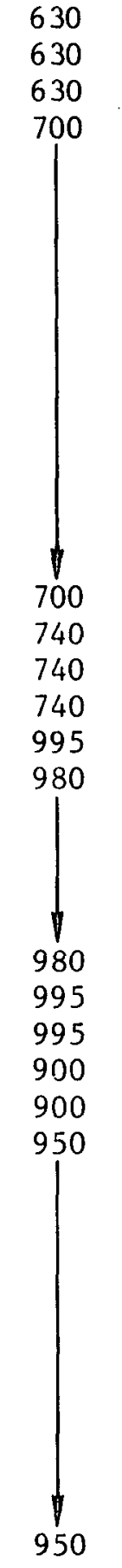 & $\begin{array}{l}-0.15 \\
-0.16 \\
-0.06 \\
-0.16 \\
-0.19 \\
-0.16 \\
-0.20 \\
-0.18 \\
-0.16 \\
-0.16 \\
-0.10 \\
-0.13 \\
-0.12 \\
-0.19 \\
-0.15 \\
-0.11 \\
-0.05 \\
-0.06 \\
-0.13 \\
-0.14 \\
-0.43 \\
-0.59 \\
-0.54 \\
-0.39 \\
-0.31 \\
-0.46 \\
-0.43 \\
-0.48 \\
-0\end{array}$ & \\
\hline
\end{tabular}


TABLE 5-9 (continued)

\begin{tabular}{|c|c|c|c|c|}
\hline $\begin{array}{c}\text { Record } \\
\text { No. }\end{array}$ & $\begin{array}{c}\text { Total } \\
\text { F1uence } \\
\left(10^{21} \mathrm{n} / \mathrm{cm}^{2}\right) \\
(\mathrm{E}>0.18 \mathrm{MeV})_{\text {HTGR }} \\
\end{array}$ & $\begin{array}{l}\text { Irrad } \\
\text { Temp } \\
\left({ }^{\circ} \mathrm{C}\right)\end{array}$ & $\begin{array}{l}\text { Irrad } \\
\text { Strain } \\
\ln \left(\ell / \ell_{0}\right) \\
\left(10^{-2}\right)\end{array}$ & $\begin{array}{c}\text { Dimensional } \\
\text { Change, } \\
\Delta \ell / \ell_{O} \\
(\%)\end{array}$ \\
\hline $\begin{array}{l}5349 \\
5348 \\
5347 \\
5346 \\
5345 \\
5344 \\
5343 \\
5342 \\
5341 \\
5340 \\
5339 \\
5338 \\
5337 \\
5442 \\
5470 \\
5460 \\
5451 \\
5481 \\
5489 \\
5486 \\
5485 \\
5482 \\
5575 \\
5518 \\
5512 \\
5505 \\
5504 \\
5501 \\
5500 \\
5497 \\
5494 \\
5493 \\
5490 \\
6471 \\
5579 \\
5587 \\
5584 \\
5583 \\
5580 \\
5588 \\
5592 \\
5591 \\
5600\end{array}$ & $\left.\right|^{3.0}$ & \begin{tabular}{l}
950 \\
950 \\
950 \\
950 \\
980 \\
\multicolumn{1}{|}{} \\
\multicolumn{1}{|}{} \\
980 \\
900 \\
1 \\
900 \\
1180 \\
1160 \\
1160 \\
1160 \\
1180 \\
1045 \\
1115 \\
1115 \\
1160 \\
11 \\
1160 \\
1385 \\
1385 \\
1385 \\
1385 \\
1395 \\
1385
\end{tabular} & $\begin{array}{l}-0.34 \\
-0.55 \\
-0.41 \\
-0.31 \\
-0.44 \\
-0.54 \\
-0.45 \\
-0.67 \\
-0.55 \\
-0.46 \\
-0.55 \\
-0.50 \\
-0.32 \\
-0.30 \\
-0.30 \\
-0.31 \\
-0.33 \\
-0.81 \\
-0.82 \\
-0.68 \\
-0.94 \\
-0.63 \\
-0.63\end{array}$ & $\begin{array}{l}-2.06 \\
-2.04 \\
-1.75 \\
-2.07 \\
-1.87 \\
-1.88 \\
-1.58 \\
-2.08 \\
-1.76\end{array}$ \\
\hline
\end{tabular}


TABLE 5-9 (continued)

\begin{tabular}{|c|c|c|c|c|}
\hline $\begin{array}{c}\text { Record } \\
\text { No. }\end{array}$ & $\begin{array}{c}\text { Total } \\
\text { F1uence } \\
\left(10^{21} \mathrm{n} / \mathrm{cm}^{2}\right) \\
(\mathrm{E}>0.18 \mathrm{MeV}) \\
\end{array}$ & $\begin{array}{l}\text { Irrad } \\
\text { Temp } \\
\left({ }^{\circ} \mathrm{C}\right)\end{array}$ & $\begin{array}{l}\text { Irrad } \\
\text { Strain } \\
\ln \left(\ell / \ell_{0}\right) \\
\left(10^{-2}\right)\end{array}$ & $\begin{array}{c}\text { Dimensional } \\
\text { Change, } \\
\Delta \ell / \ell_{0} \\
(\%)\end{array}$ \\
\hline $\begin{array}{l}5599 \\
5596 \\
5595 \\
5603 \\
5606 \\
5604 \\
5674 \\
5612 \\
5610 \\
5608 \\
5691 \\
5689 \\
5695 \\
5694 \\
5703 \\
5702 \\
5699 \\
5698 \\
5721 \\
5720 \\
5717 \\
5716 \\
5713 \\
5712 \\
5709 \\
5708 \\
5724 \\
5778 \\
5774 \\
5765 \\
5733 \\
5732 \\
5729 \\
5728 \\
5725 \\
5802 \\
5800 \\
5796 \\
5794 \\
5790 \\
5787 \\
5783 \\
5820\end{array}$ & $\left.\right|^{3.2}$ & 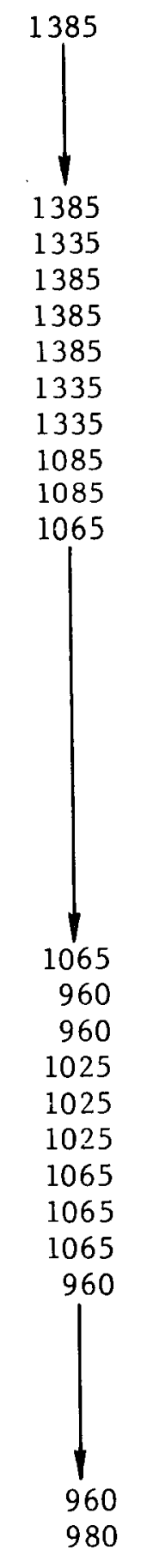 & $\begin{array}{l}-2.00 \\
-1.94 \\
-2.04 \\
-2.10 \\
-1.97 \\
-2.03 \\
-1.60 \\
-1.87 \\
-1.07 \\
-1.04 \\
-1.47 \\
-1.74 \\
-0.53 \\
-0.60 \\
-0.50 \\
-0.61 \\
-0 \\
-0.49 \\
-0.64 \\
-0.49 \\
-0\end{array}$ & $\begin{array}{l}-1.98 \\
-1.92 \\
-2.02 \\
-2.08 \\
-1.95 \\
-2.01 \\
-1.58 \\
-1.85 \\
-2.00 \\
-1.62 \\
-1.46 \\
-1.74\end{array}$ \\
\hline
\end{tabular}


TABLE 5-9 (continued)

\begin{tabular}{|c|c|c|c|c|}
\hline $\begin{array}{c}\text { Record } \\
\text { No. }\end{array}$ & $\begin{array}{c}\text { Total } \\
\text { Fluence } \\
\left(1021 \mathrm{n} / \mathrm{cm}^{2}\right) \\
(E>0.18 \mathrm{MeV})_{\text {HTGR }}\end{array}$ & $\begin{array}{c}\text { Irrad } \\
\text { Temp } \\
\left({ }^{\circ} \mathrm{C}\right)\end{array}$ & $\begin{array}{l}\text { Irrad } \\
\text { Strain } \\
\ln \left(\ell / \ell_{0}\right) \\
\left(10^{-2}\right)\end{array}$ & $\begin{array}{c}\text { Dimensional } \\
\text { Change, } \\
\Delta \ell / \ell_{0} \\
(\%)\end{array}$ \\
\hline $\begin{array}{l}5816 \\
5812 \\
5808 \\
5855 \\
5853 \\
5851 \\
5849 \\
5848 \\
5845 \\
5844 \\
5841 \\
5840 \\
5837 \\
5836 \\
5833 \\
5832 \\
5829 \\
5828 \\
5824 \\
5869 \\
5905 \\
5903 \\
5901 \\
5899 \\
5898 \\
5880 \\
5892 \\
5890 \\
6045 \\
6047 \\
6043 \\
6041 \\
6023 \\
6022 \\
5941 \\
5908 \\
5916 \\
5912 \\
5940 \\
5937 \\
5936 \\
5933 \\
5932\end{array}$ & $\left.\right|^{2.8}$ & $\left.\right|_{950} ^{980}$ & $\begin{array}{l}-0.41 \\
-0.38 \\
-0.41 \\
-0.35 \\
-0.20 \\
-0.50 \\
-0.23 \\
-0.36 \\
-0.14 \\
-0.30 \\
-0.26 \\
-0.34 \\
-0.32 \\
-0.40 \\
-0.42 \\
-0.37 \\
-0.16 \\
-0.51 \\
-0.40 \\
-0.31 \\
-0.23 \\
-0.24 \\
-0.25 \\
-0.32 \\
-0.27 \\
-0.22 \\
-0.24 \\
-0.27 \\
-0.18 \\
-0.13 \\
-0.10 \\
-0.11 \\
-0.15 \\
-0.09 \\
-0.04 \\
-0.18 \\
-0.22 \\
-0.21 \\
-0.18 \\
-0.20 \\
-0.25 \\
-0.17 \\
-0.19\end{array}$ & \\
\hline
\end{tabular}


TABLE 5-9 (continued)

\begin{tabular}{|c|c|c|c|c|}
\hline $\begin{array}{c}\text { Record } \\
\text { No. }\end{array}$ & $\begin{array}{c}\text { Total } \\
\text { F1uence } \\
\left(10^{21} \mathrm{n} / \mathrm{cm}^{2}\right) \\
(\mathrm{E}>0.18 \mathrm{MeV})_{\mathrm{HTGR}}\end{array}$ & $\begin{array}{l}\text { Irrad } \\
\text { Temp } \\
\left({ }^{\circ} \mathrm{C}\right)\end{array}$ & $\begin{array}{l}\text { Irrad } \\
\text { Strain } \\
\ln \left(\ell / \ell_{0}\right) \\
\left(10^{-2}\right)\end{array}$ & $\begin{array}{c}\text { Dimensional } \\
\text { Change, } \\
\Delta \ell / \ell_{0} \\
(\%)\end{array}$ \\
\hline 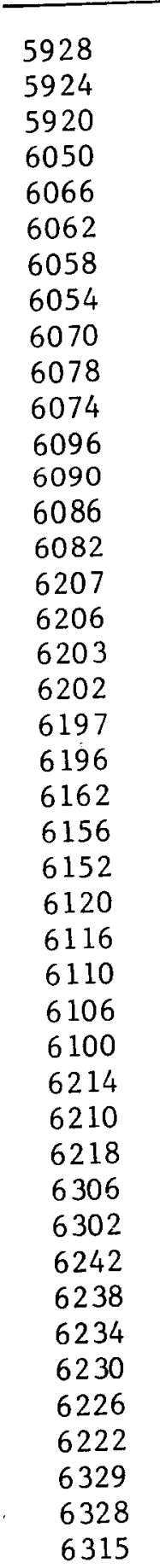 & $\left.\right|^{2.3}$ & 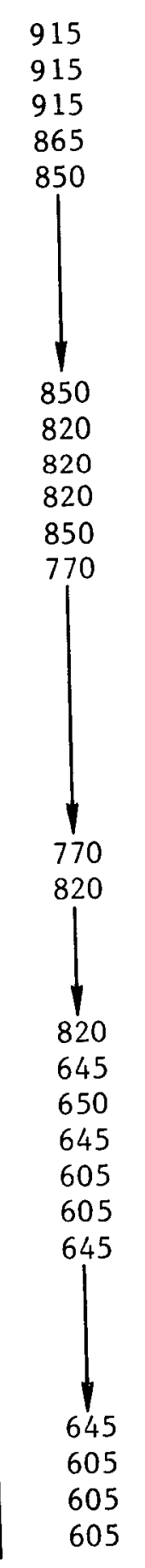 & $\begin{array}{l}-0.28 \\
-0.22 \\
-0.23 \\
-0.12 \\
-0.08 \\
-0.10 \\
-0.04 \\
-0.09 \\
-0.07 \\
-0.05 \\
-0.06 \\
-0.09 \\
-0.03 \\
-0.12 \\
-0.08 \\
-0.08 \\
-0.14 \\
-0.06 \\
-0.09 \\
-0.12 \\
-0.08 \\
-0.12 \\
-0.03 \\
-0.07 \\
-0.12 \\
-0.07 \\
-0.03 \\
-0.04 \\
-0.10 \\
-0.03 \\
-0.03 \\
-0.07 \\
-0.15 \\
-0.13 \\
-0.02 \\
-0.11 \\
-0.12 \\
-0.04 \\
-0.06 \\
-0.05 \\
-0.06 \\
-0.15 \\
-0.03\end{array}$ & \\
\hline
\end{tabular}


TABLE 5-9 (continued)

\begin{tabular}{|c|c|c|c|c|}
\hline $\begin{array}{c}\text { Record } \\
\text { No. }\end{array}$ & $\begin{array}{c}\text { Total } \\
\text { Fluence } \\
\left(10^{21} \mathrm{n} / \mathrm{cm}^{2}\right) \\
(E>0.18 \mathrm{MeV})_{\text {HTGR }}\end{array}$ & $\begin{array}{l}\text { Irrad } \\
\text { Temp } \\
\left({ }^{\circ} \mathrm{C}\right)\end{array}$ & $\begin{array}{l}\text { Irrad } \\
\text { Strain } \\
\ln (\ell / \ell) \\
\left(10^{-2}\right)\end{array}$ & $\begin{array}{c}\text { Dimensional } \\
\text { Change, } \\
\Delta \ell / \ell_{0} \\
(\%)\end{array}$ \\
\hline $\begin{array}{l}6314 \\
6307 \\
6325 \\
6324 \\
6319 \\
6318\end{array}$ & $\left.\right|_{1.4} ^{1.4}$ & $\int_{605}^{605}$ & $\begin{array}{l}-0.11 \\
-0.10 \\
-0.04 \\
-0.06 \\
-0.14 \\
-0.05\end{array}$ & \\
\hline
\end{tabular}


TABLE 5-10

DTMENSIONAL CHARGE DATA, H-451, RADIAL DIRECTION,

DUMARSIONAL CHANOE DATA, HULE OG-?

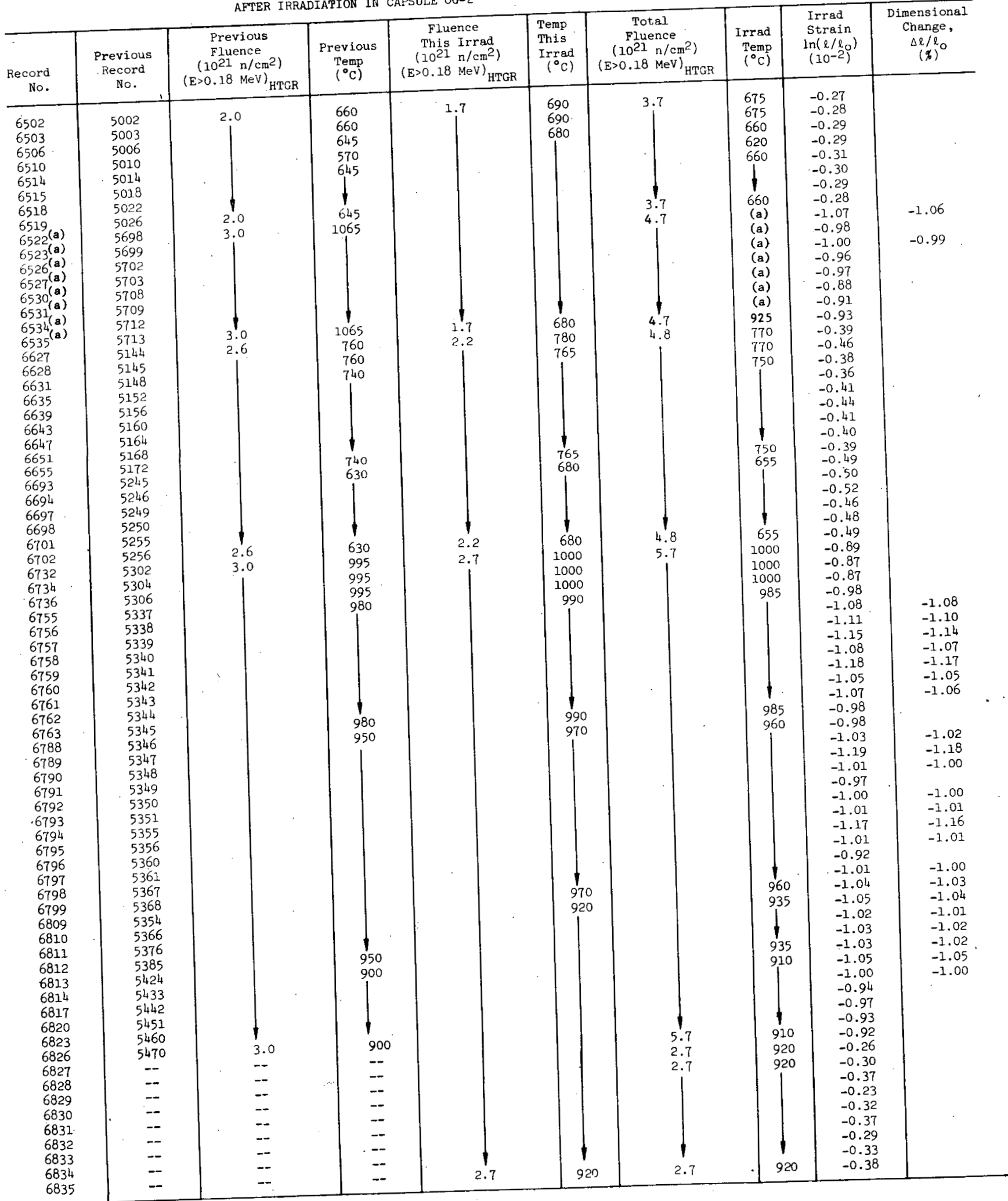




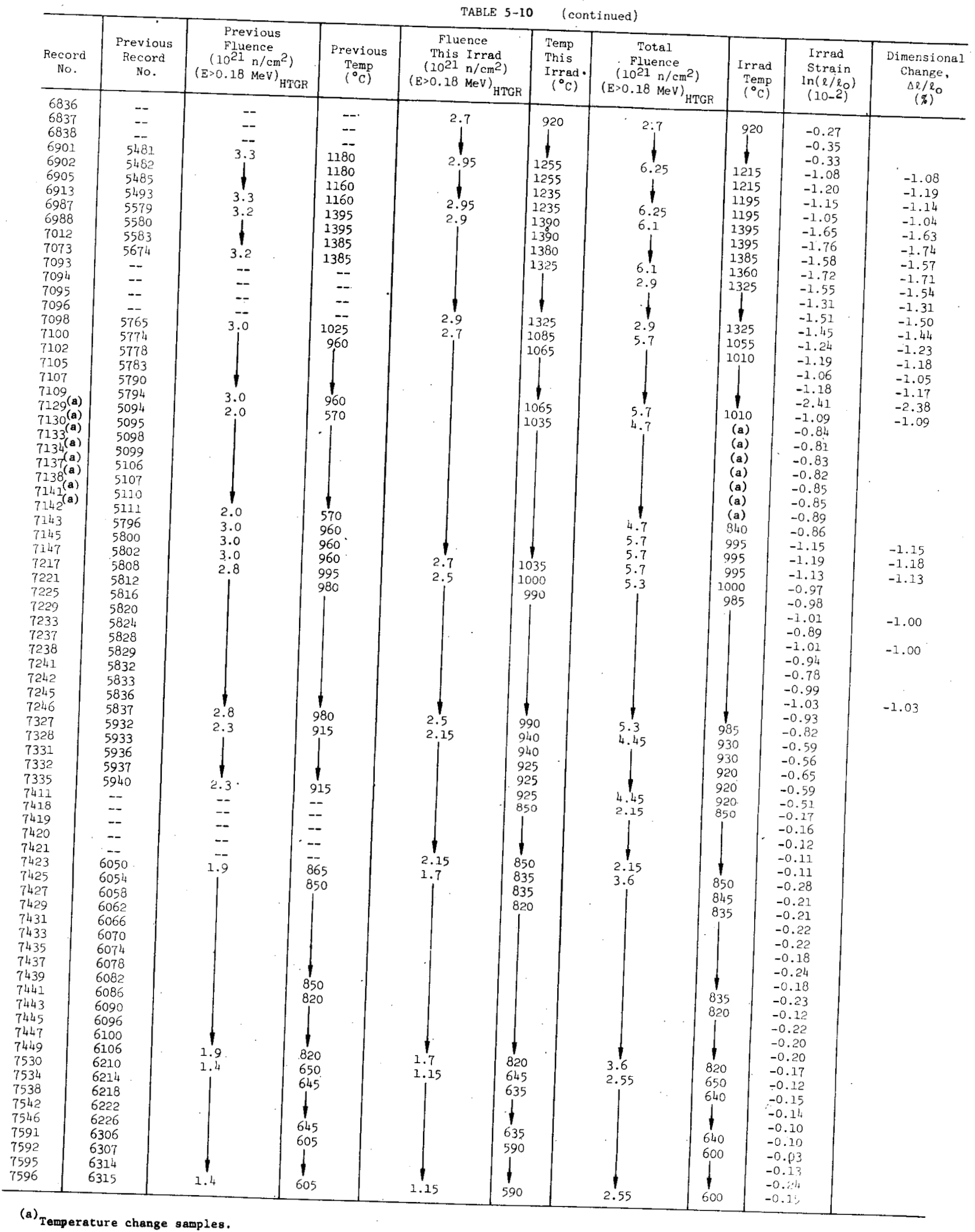




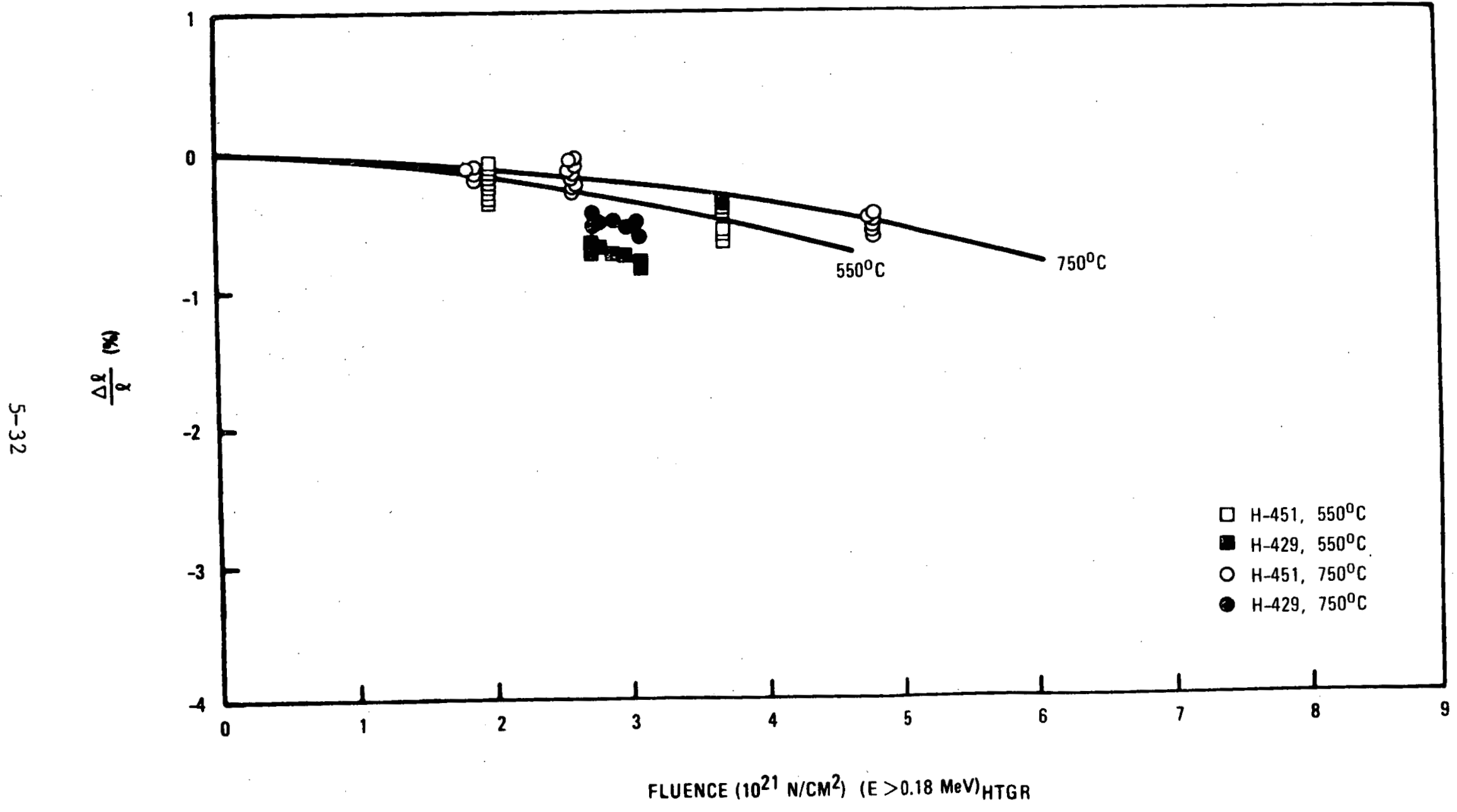

Fig. 5-1(a). Dimensional change of H-451/H-429 graphite, axial orientation, as a function of fast neutron fluence at irradiation temperatures of $550^{\circ}$ and $750^{\circ} \mathrm{C}$ 


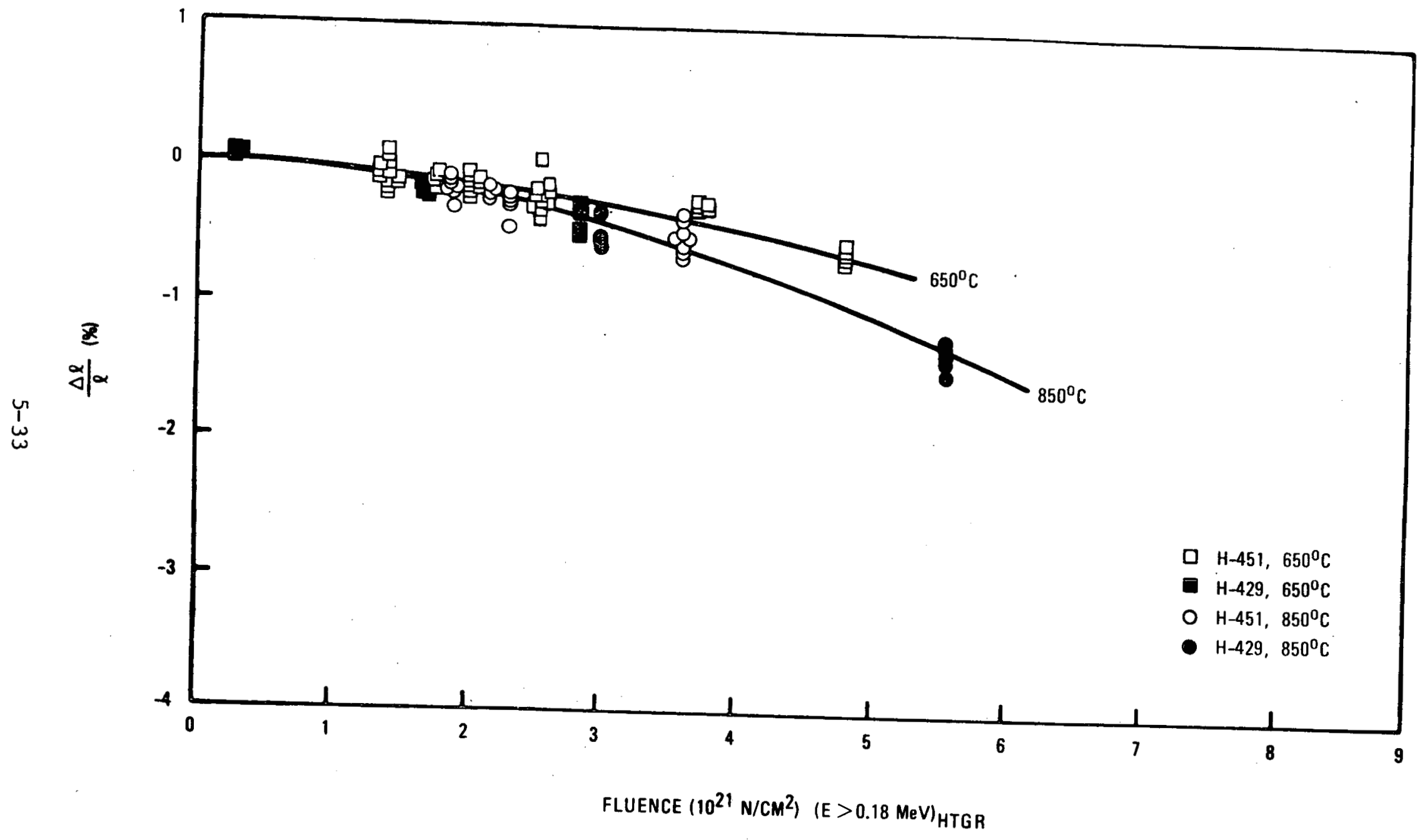

Fig. 5-1(b). Dimensional change of H-451/H-429 graphite, axial orientation, as a function of fast
neutron fluence at irradiation temperatures of $650^{\circ}$ and $850^{\circ} \mathrm{C}$ 


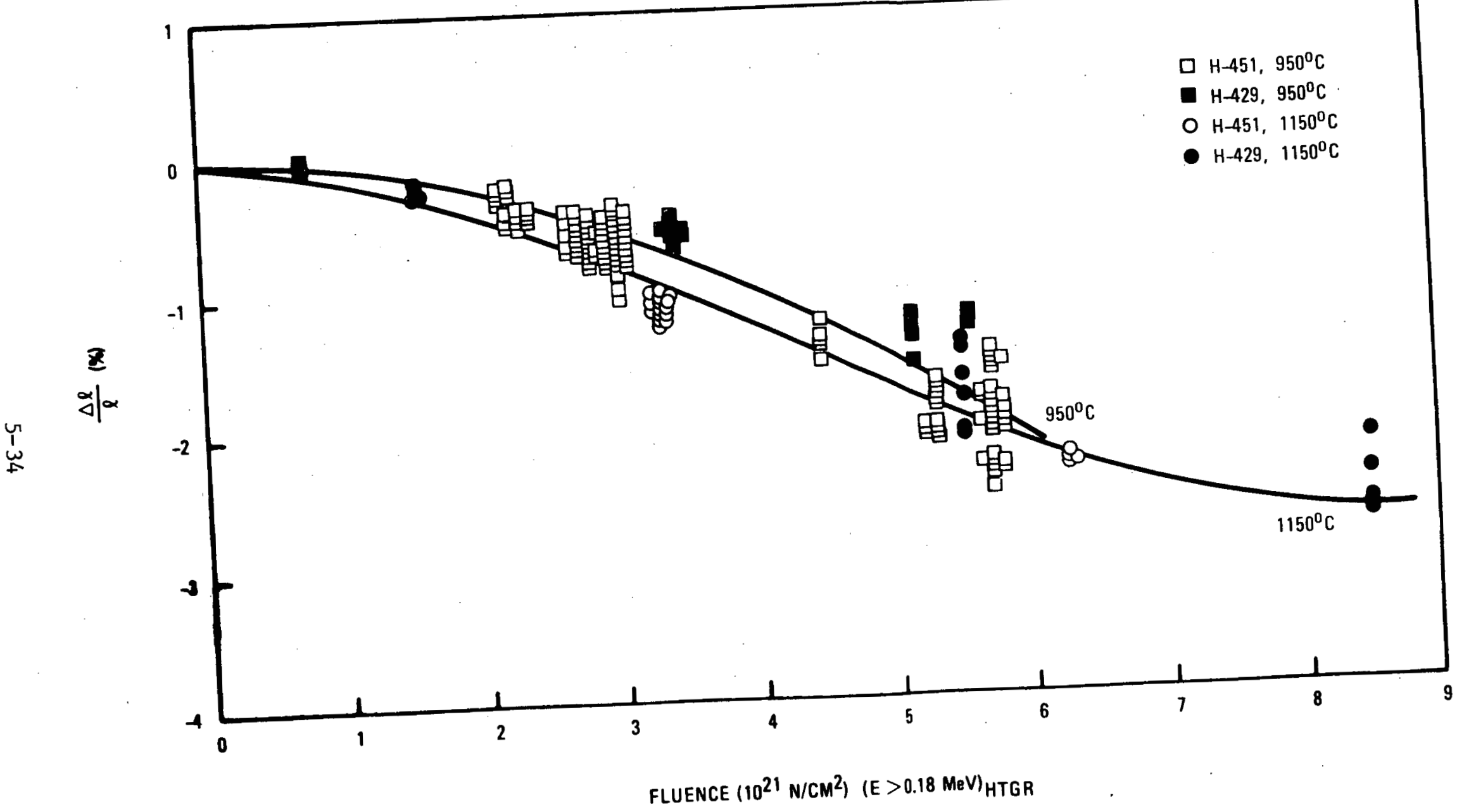

Fig. 5-1(c). Dimensional change of H-451/H-429 graphite, axial orientation, 


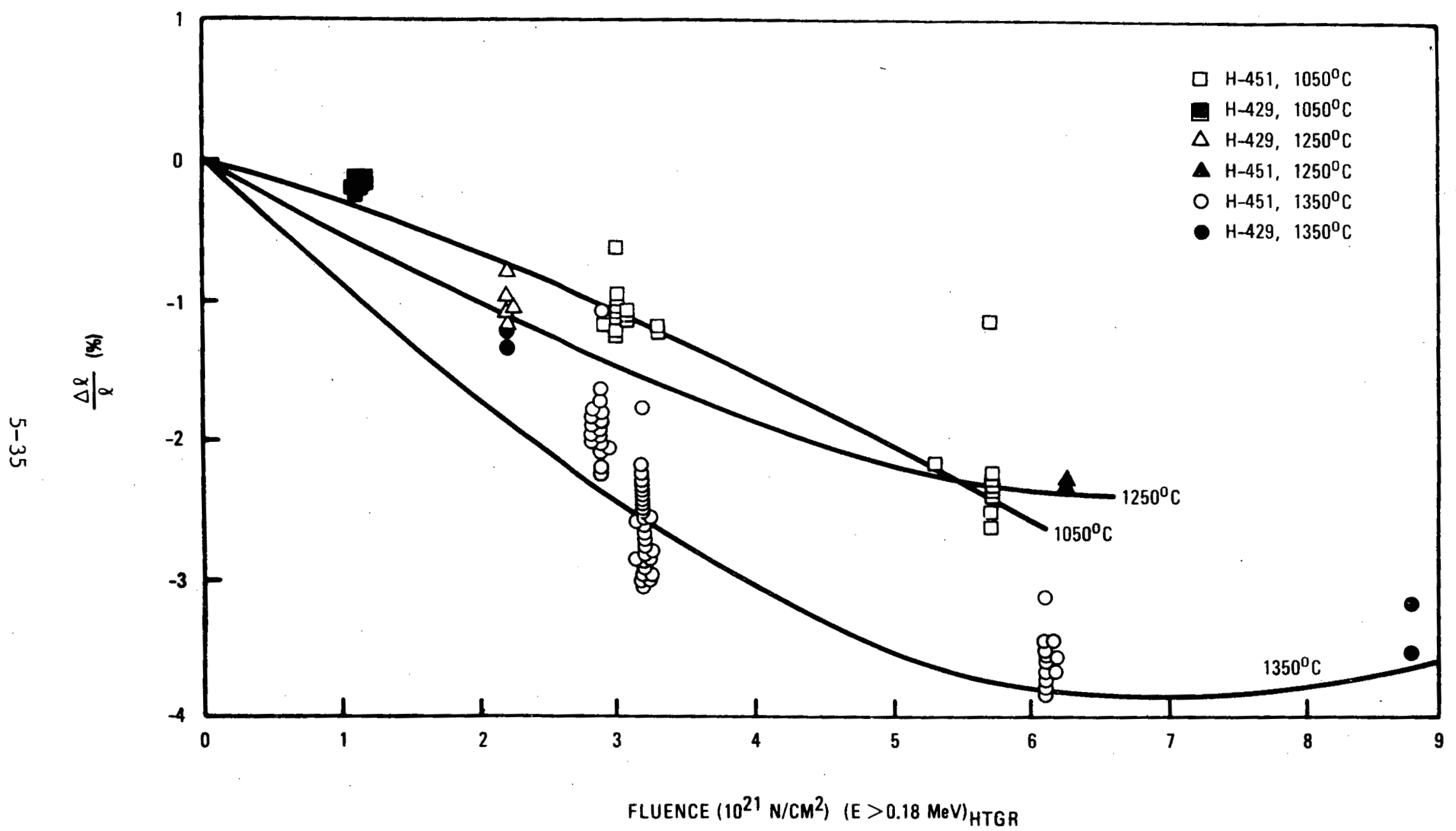

Fig. 5-1(d). Dimensional change of H-451/H-429 graphite, axial orientation, as a function of fast neutron fluence at irradiation temperatures of $1050^{\circ}, 1250^{\circ}$, and $1350^{\circ} \mathrm{C}$ 


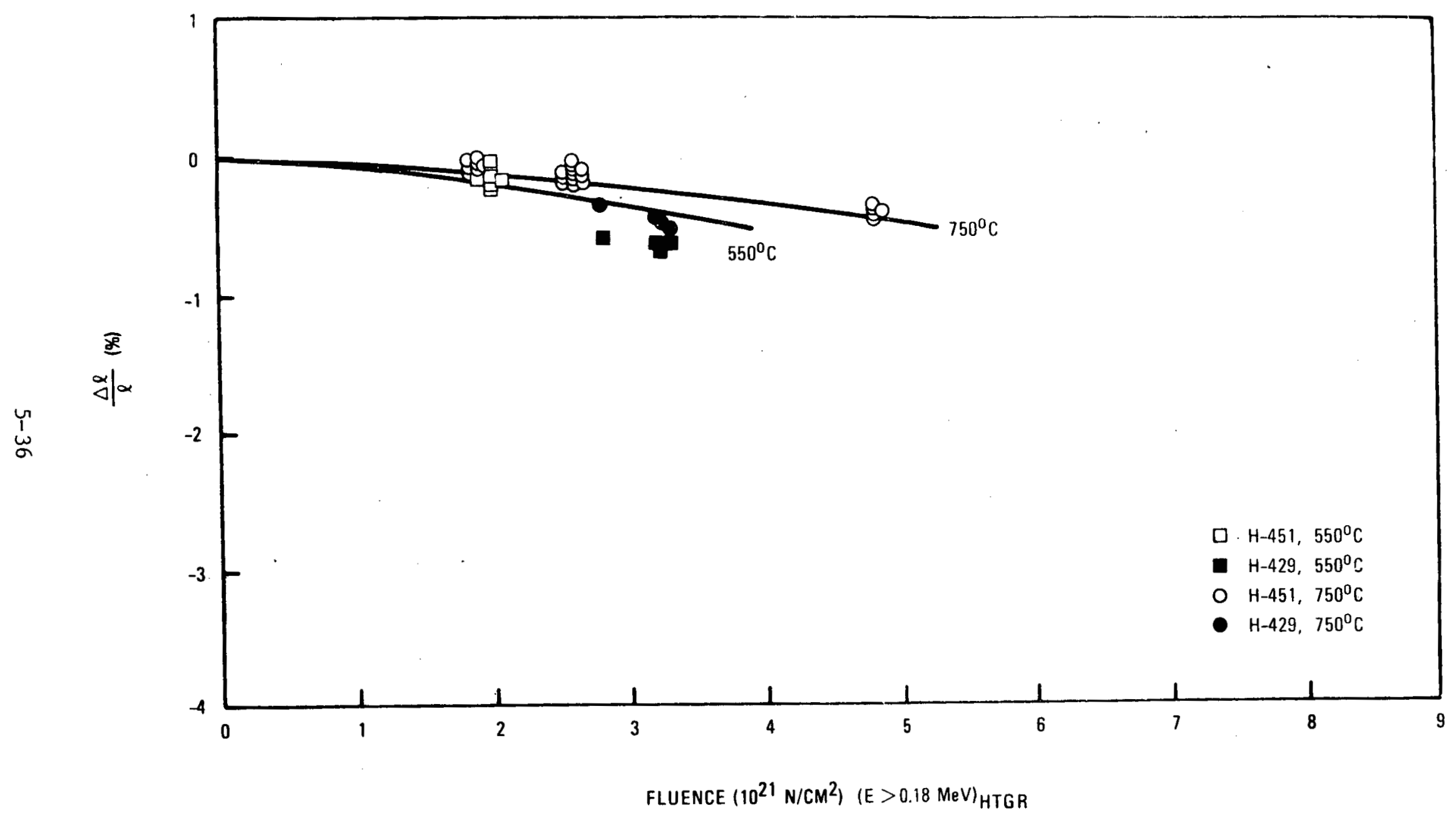

Fig. 5-2(a). Dimensional change of $\mathrm{H}-451 / \mathrm{H}-429$ graphite, radial orientation, as a function of fast neutron fluence at irradiation temperatures of $550^{\circ}$ and $750^{\circ} \mathrm{C}$ 
c

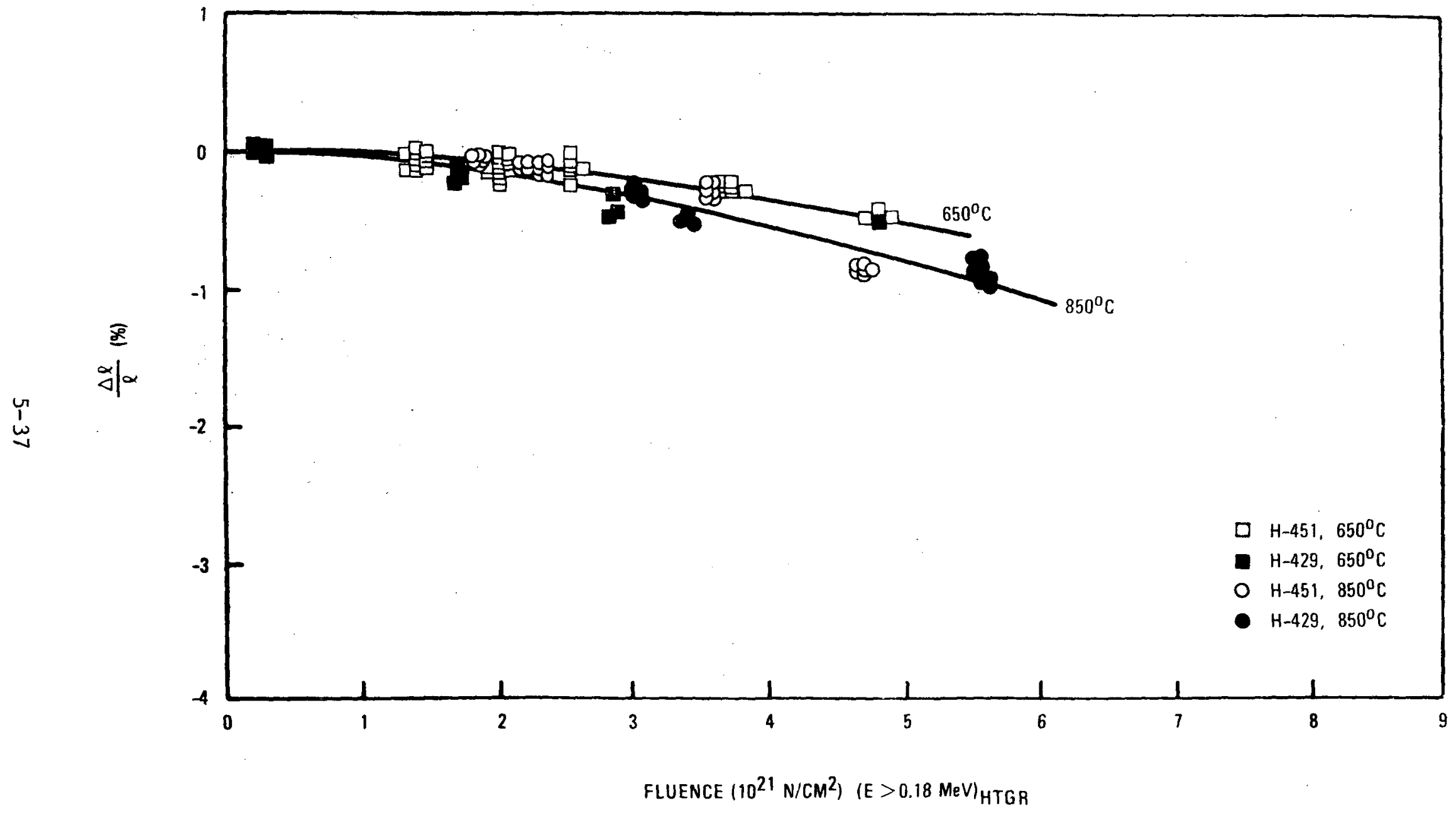

Fig. 5-2(b). Dimensional change of H-451/H-429 graphite, radial orientation, as a function of fast neutron fluence at irradiation temperatures of $650^{\circ}$ and $850^{\circ} \mathrm{C}$ 


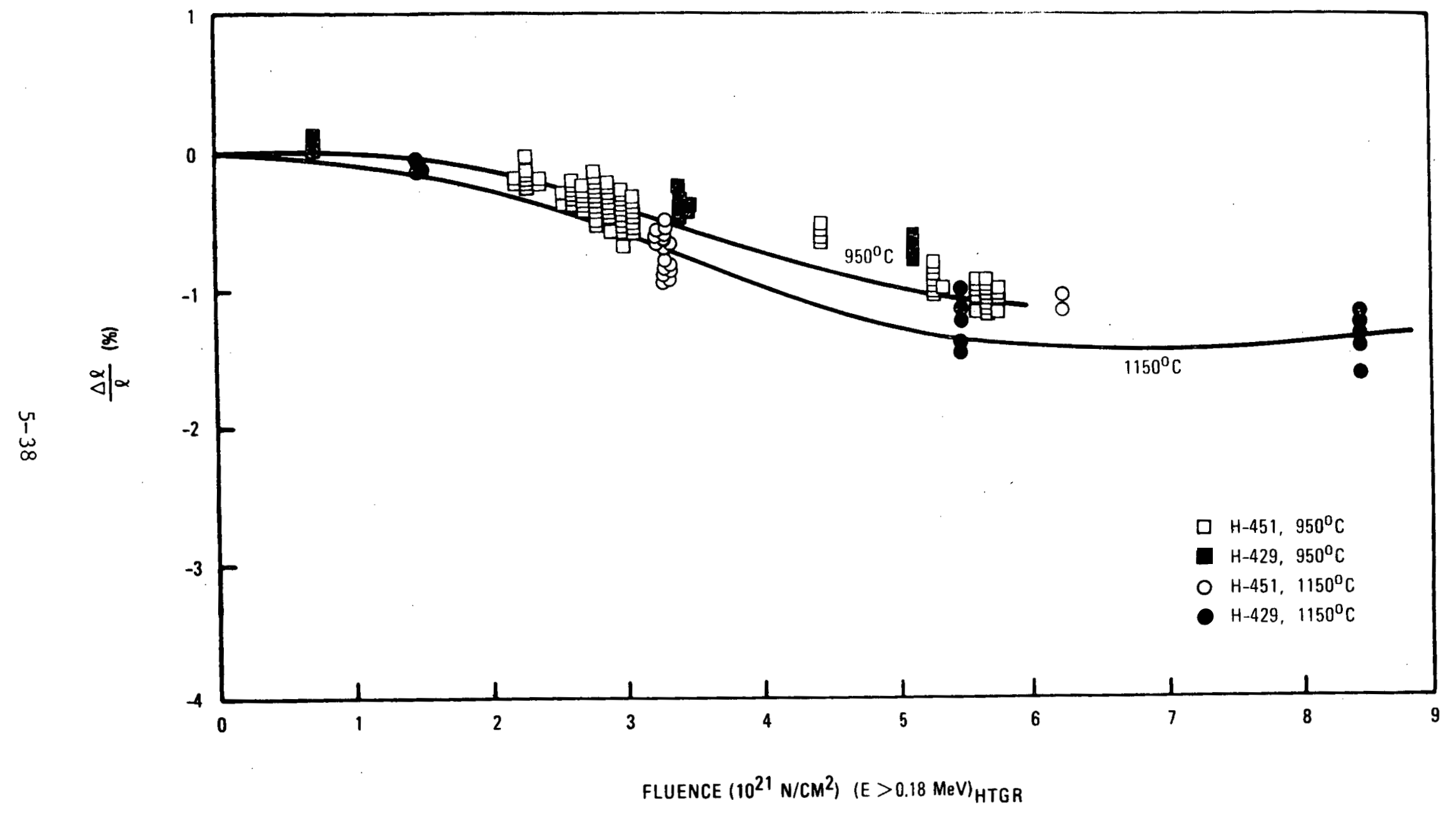
Fig. 5-2(c). Dimensional change of $\mathrm{H}-451 / \mathrm{H}-429$ graphite, radial orientation, as a function of fast
neutron fluence at irradiation temperatures of $950^{\circ}$ and $1150^{\circ} \mathrm{C}$ 


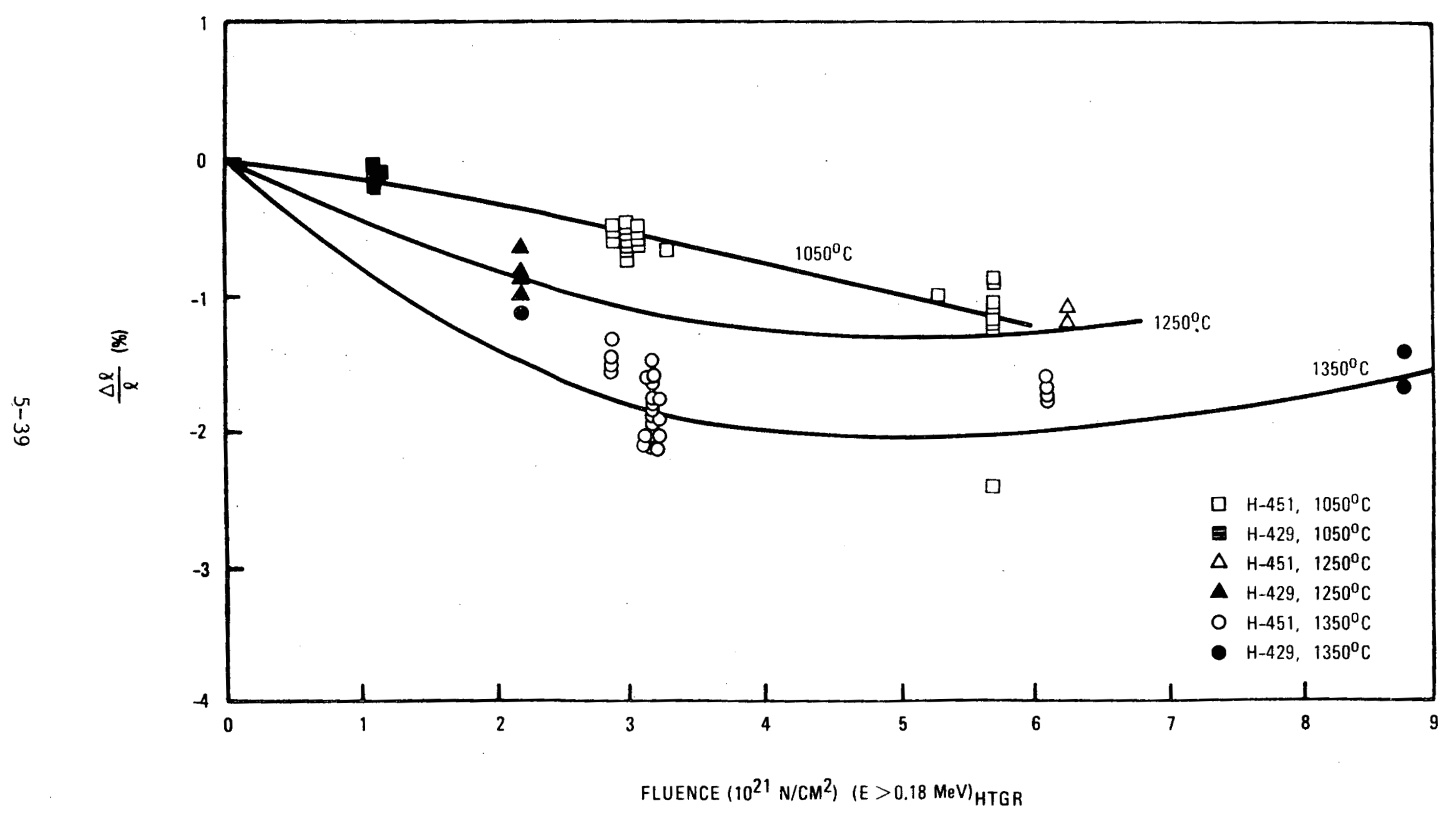

Fig. 5-2(d). Dimensional change of $\mathrm{H}-451 / \mathrm{H}-429$ graphite, radial orientation, as a function of fast neutron fluence at irradiation temperatures of $1050^{\circ}, 1250^{\circ}$, and $1350^{\circ} \mathrm{C}$ 
Design curves of the dimensional change in near-isotropic graphite ( $\mathrm{H}-451 / \mathrm{H}-429)$ were derived from the data in the following manner:

1. The data of Tables 5-1 through 5-10 (shown in Figs. 5-1 and 5-2) were tabulated, reviewed, and input to a computer-fitting code.

2. Supplementary 10w-temperature $\left(300^{\circ}-600^{\circ} \mathrm{C}\right)$, high-fluence data from Gilsocoke graphites from Ref. 6 were added to the data base for the $300^{\circ}$ to $600^{\circ} \mathrm{C}$ range.

3. The computer code fit a surface through the three-dimensional data space (fluence, temperature, dimensional change) by a leastsquares, Grahm-Schmitt orthogonalization process. One surface was generated for each orientation of graphite specimens.

4. Isotherms were taken from the computer-fit surface, compared with the actual data, and then plotted in Figs. 5-3 and 5-4 to represent the design curves. A polynomial generated by the computer fit descriuing the surface is actually used for design work.

The resulting surface and its isotherms plotted in Figs. 5-3 and 5-4 differ somewhat from earlier design curves because of the change in fast fluence units. The shift of approximately $11 \%$ along the fluence axis was observed to be larger than the shift in the curve resulting from the addition of OG-2 data. The design curves plotted in ORR fluence units (Ref. 4) shifted approximately $5 \%$ with the addition of OG-2 data in the same ORR units.

The data in Figs. 5-1 through 5-4 illustrate the dimensional stability of $\mathrm{H}-451$ under neutron irradiation. These data have been obtained at sufficiently high fluences to demonstrate that the contraction of $\mathrm{H}-451$ will be $<2.0 \%$ radially and $<3.0 \%$ axially during the graphite residence time in a large HTGR core. 


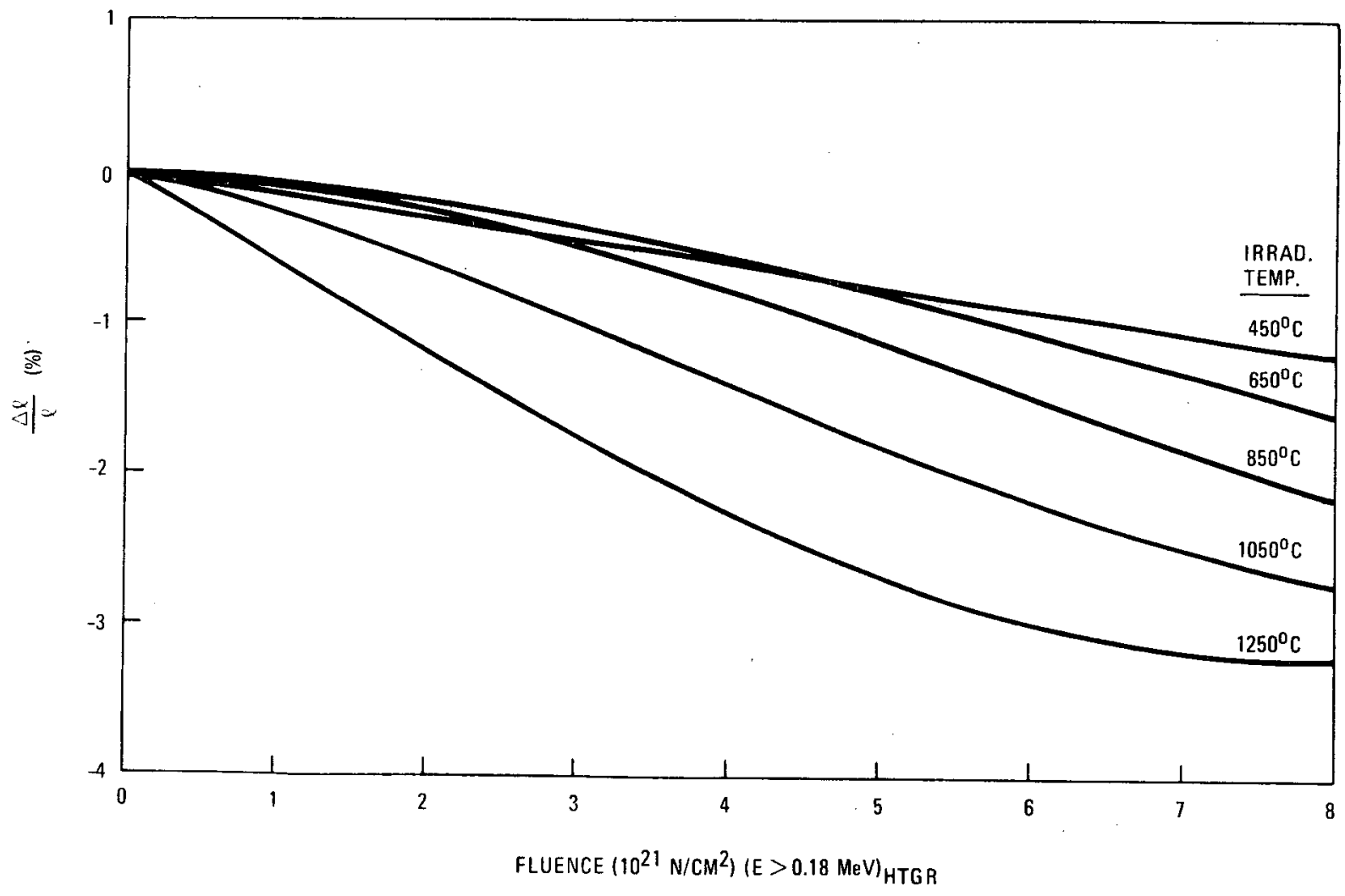

Fig. 5-3. Design curves for irradiation-induced axial dimensional change in $\mathrm{H}-451 / \mathrm{H}-429$ graphite 


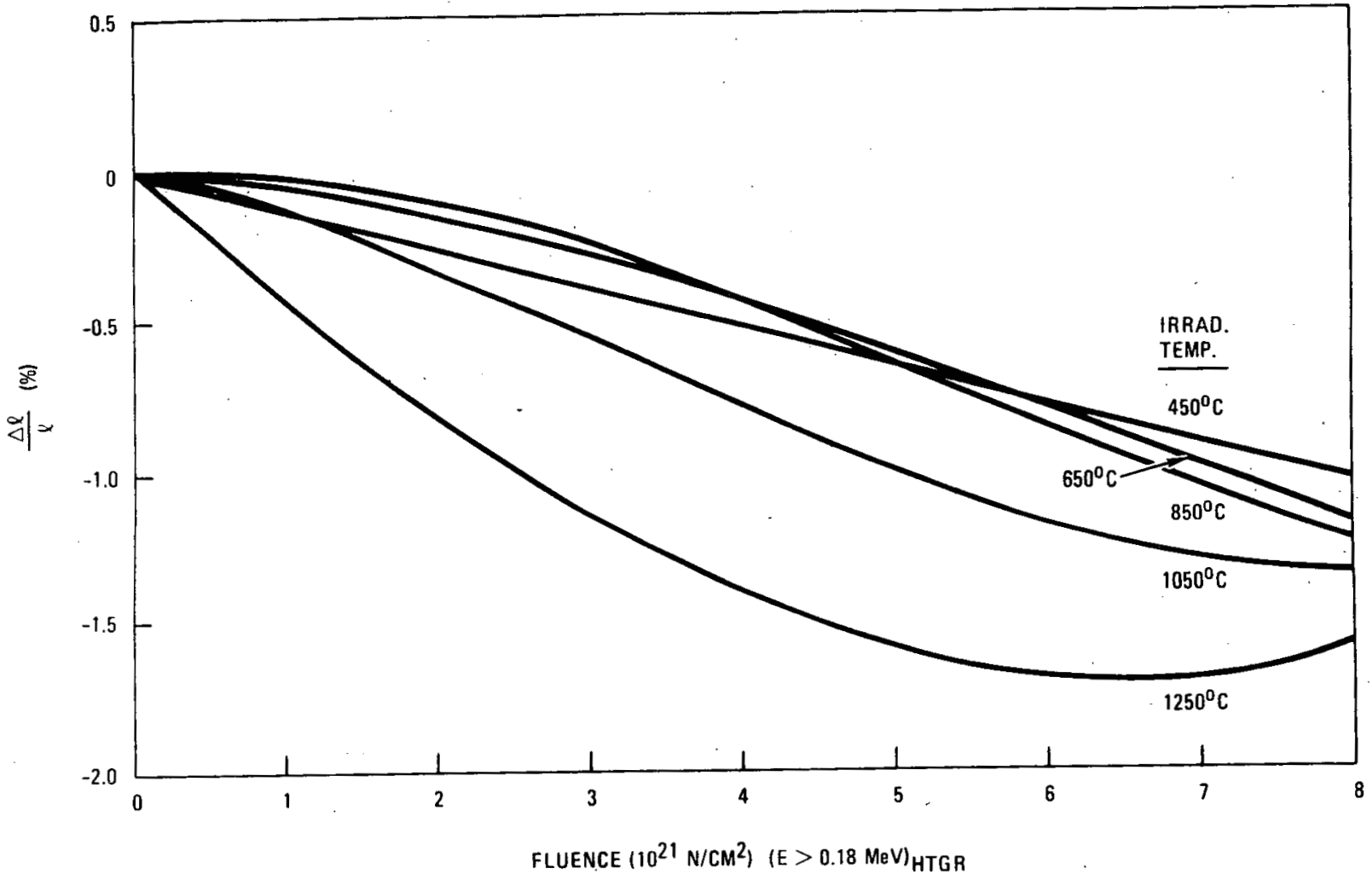

Fig. 5-4. Design curves for irradiation-induced radial dimensional change in $\mathrm{H}-451 / \mathrm{H}-429$ graphite 
Specimens of $\mathrm{H}-451$ were irradiated in $O G-1$ at $T_{1}$ and subsequently irradiated in $O G-2$ at $\mathrm{T}_{2}$. The following data sets are plotted in Fig. 5-5 along with isothermal curves:

$$
\begin{aligned}
& \text { 1. } \mathrm{T}_{1}=570^{\circ} \mathrm{C}, \mathrm{T}_{2}=1035^{\circ} \mathrm{C} \text {, isotherm at } 650^{\circ} \mathrm{C} \text {. } \\
& \text { 2. } \mathrm{T}_{1}=1065^{\circ} \mathrm{C}, \mathrm{T}_{2}=680^{\circ} \mathrm{C} \text {, isotherm at } 1050^{\circ} \mathrm{C} \text {. }
\end{aligned}
$$

When the specimens were irradiated first at $570^{\circ} \mathrm{C}$ and then at $1035^{\circ} \mathrm{C}$, the contraction rate increased during the $1035^{\circ} \mathrm{C}$ increment and equaled the isothermal rate at $1050^{\circ} \mathrm{C}$. When the specimens were irradiated first at $1065^{\circ} \mathrm{C}$ and then at $680^{\circ} \mathrm{C}$, the contraction rate decreased for the axial specimens but remained unchanged for the radial specimens. There is not a sufficient quantity of data to permit further conclusions at this time. These specimens will be irradiated to higher fluences at $\mathrm{T}_{2}$.

Axial H-451 specimens irradiated side by side in OG-2 showed midlength edge (MLE) specimens to contract by $10 \%$ to $20 \%$ less than midlength center (MLC) specimens. These data are shown in Table 5-11 for $\mathrm{H}-451$ graphite. No size effect has been discerned for the two sizes of specimens irradiated.

The TS-1240 graphite specimens irradiated for the first time in OG-2 exhibited excellent dimensional stability. None of the specimens contracted more than $1 \%$ up to fast fluences of $2.95 \times 10^{21} \mathrm{n} / \mathrm{cm}^{2}$ ( $\left.\mathrm{E}>0.18 \mathrm{MeV}\right)_{\text {HTGR }}$ and temperatures of $1105^{\circ} \mathrm{C}$. These data are given in Tables 5-12 and 5-13.

$\mathrm{P}_{3}$ JHAN graphite specimens carried over from the $O G-1$ experiment continued to show high contraction in the axial orientation. In the radial direction, specimens at several sets of irradiation conditions went through turnaround to net expansion. Tables 5-14 through 5-17 give data for $\mathrm{P}_{3}$ JHAN irradiated in capsules $O G-1$ and $O G-2$.

\subsection{DIMENSIONAL CHANGE IN NEEDLE COKE GRAPHITES}

Samples of H-327 graphite were carried over from the OG-1 experiment for further neutron exposure. As reported earlier (Ref. 4), the H-327

$$
5-43
$$




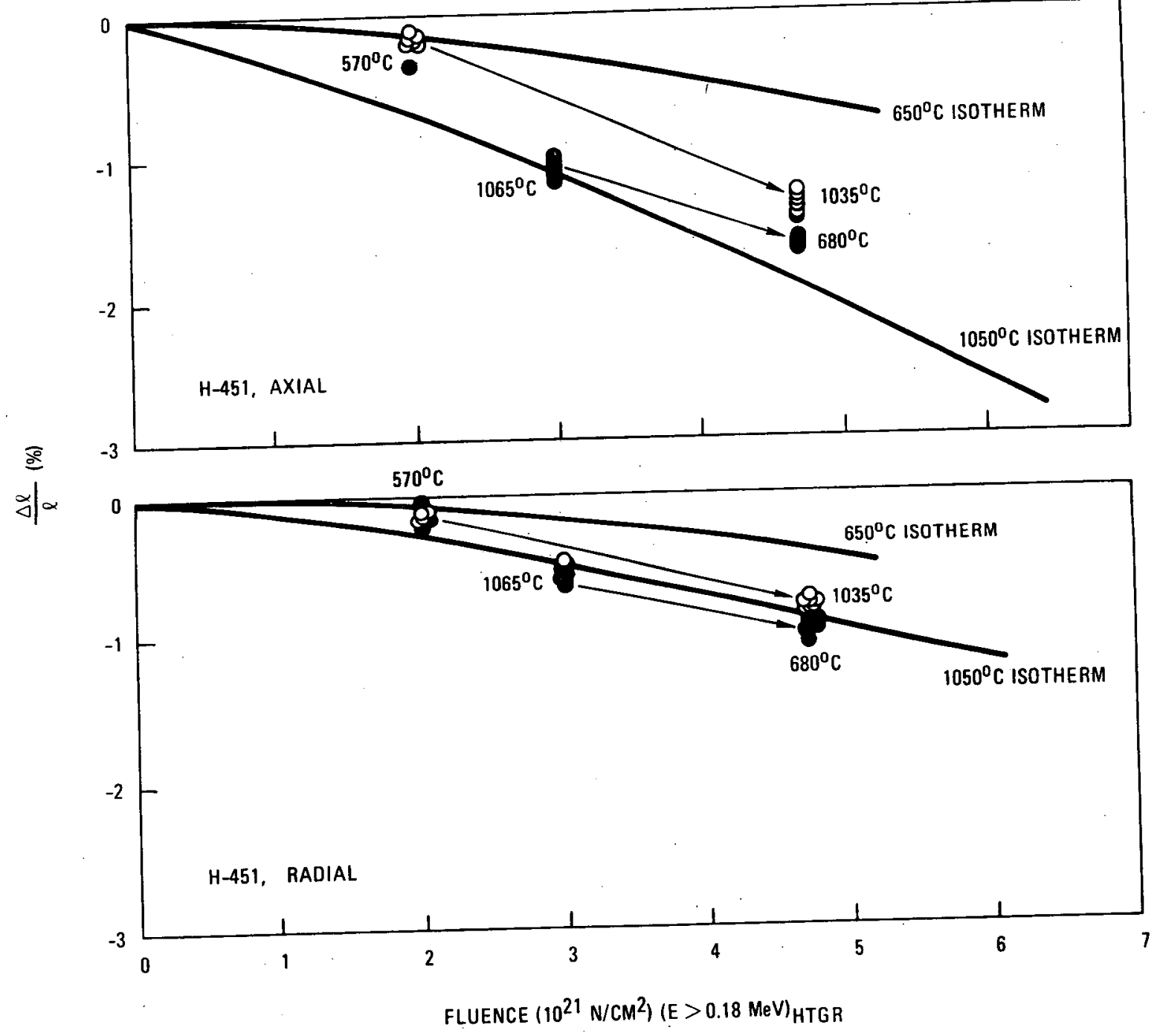

Fig. 5-5. Dimensional change in $\mathrm{H}-451$ graphite for non-isothermal irradiation 
TABLE $5-11$

COMPARISON OF DIMENSTONAL CHANGES IN MIDLENGTH CENTER (MLC)

AND MIDLENGTH EDGE (MLE) H-451. SPECIMENS

\begin{tabular}{|c|c|c|c|c|c|c|c|c|}
\hline \multirow[b]{2}{*}{$\begin{array}{l}\text { Record } \\
\text { Numbers }\end{array}$} & \multicolumn{2}{|c|}{ Irradiation Conditions } & \multirow[b]{2}{*}{$\begin{array}{c}\text { No. } \\
\text { Samples }\end{array}$} & \multirow[b]{2}{*}{ Location } & \multirow[b]{2}{*}{ Orientation } & \multicolumn{2}{|c|}{ Dimensional Change, $\ln \left(\ell_{n} / \ell_{0}\right)$} & \multirow[b]{2}{*}{$\begin{array}{c}\text { Difference in } \\
\text { Dimensional Change } \\
\text { between MLC and MLE Samples } \\
(\%)\end{array}$} \\
\hline & $\begin{array}{c}\text { Fluence } \\
\left(10^{21} \mathrm{n} / \mathrm{cm}^{2}\right) \\
(\mathrm{E}>0.18 \mathrm{MeV})_{\text {HTGR }}\end{array}$ & $\begin{array}{l}\text { Temp. } \\
\left({ }^{\circ} \mathrm{C}\right)\end{array}$ & & & & $\left(10^{\left.-2^{\text {Mean }} \text { in./in. }\right)}\right.$ & $\frac{\text { ange, } \ln \left(x_{n} / x_{0}\right)}{\left(10^{-2} \text { in. Dev. }\right.}$ & \\
\hline $7215-7246$ & 5.2 & 990 & 11 & MLE & Axial & -2.08 & 0.09 & \\
\hline $7099-7110$ & $5.2^{(a)}$ & 1010 & 6 & MLC & Axial & $-2.17^{(a)}$ & 0.11 & $-4 \cdot 3$ \\
\hline $7217-7246$ & 5.2 & 990 & 11 & MLE & Radial & -0.94 & 0.08 & \\
\hline $7143-7147$ & $5.2^{(a)}$ & 995 & 3 & MLC & Radial &.$-1.06^{(a)}$ & 0.03 & -11.3 \\
\hline $7361-7372$ & 2.15 & 905 & 6 & MLE & Axial & -0.24 & 0.02 & \\
\hline $7361-7372$ & 2.15 & 905 & 6 & MLC & Axial & -0.31 & 0.02 & -22.6 \\
\hline $7410-7421$ & 2.15 & 850 & 3 & MLE & Axial & -0.20 & 0.03 & . \\
\hline $7410-7421$ & 2.15 & 850 & 4 & MLC & Axial & -0.25 & 0.02 & -20.0 \\
\hline
\end{tabular}

(a) Actual measurements made at a fluence of $5.7 \times 10^{21} \mathrm{n} / \mathrm{cm}^{2}$. Dimensional change values at $5.2 \times 10^{21} \mathrm{n} / \mathrm{cm}^{2}$ were $\mathrm{calculated}$ by linear interpolation between 0 and $5.7 \times 10^{21} \mathrm{n} / \mathrm{cm}^{2}$. 
TABLE 5-12

DIMENSIONAL CHANGE DATA, TS-1240, AXIAL DIRECTION, AFTER IRRADIATION IN CAPSULE OG-2

\begin{tabular}{|c|c|c|c|}
\hline $\begin{array}{c}\text { Record } \\
\text { No. }\end{array}$ & $\begin{array}{c}\text { Total } \\
\text { Fluence } \\
\left(10^{21} \mathrm{n} / \mathrm{cm}^{2}\right) \\
(E>0.18 \mathrm{MeV})_{\text {HTGR }}\end{array}$ & $\begin{array}{l}\text { Irrad } \\
\text { Temp } \\
\left({ }^{\circ} \mathrm{C}\right)\end{array}$ & $\begin{array}{l}\text { Irrad } \\
\text { Strain } \\
\ln (\ell / \ell O) \\
(10-2)\end{array}$ \\
\hline 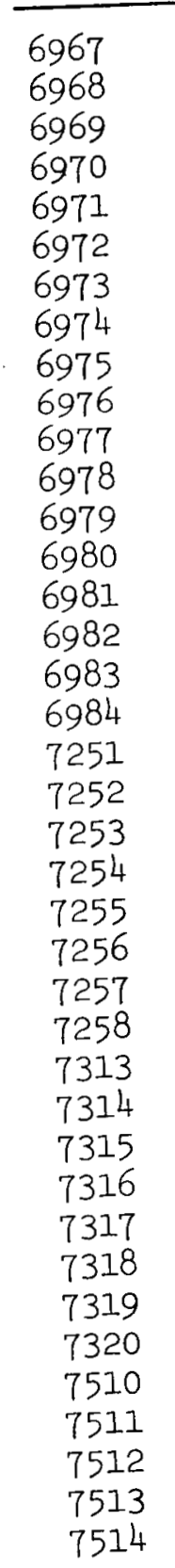 & $\left.\right|^{2.95}$ & $\begin{array}{l}\mid \\
1105 \\
970 \\
1 \\
970 \\
920 \\
1 \\
920 \\
765 \\
165\end{array}$ & $\begin{array}{l}-0.78 \\
-0.82 \\
-0.67 \\
-0.74 \\
-0.65 \\
-0.72 \\
-0.66 \\
-0.72 \\
-0.69 \\
-0.75 \\
-0.72 \\
-0.76 \\
-0.64 \\
-0.74 \\
-0.68 \\
-0.73 \\
-0.76 \\
-0.83 \\
-0.31 \\
-0.24 \\
-0.26 \\
-0.21 \\
-0.27 \\
-0.24 \\
-0.26 \\
-0.20 \\
-0.23 \\
-0.25 \\
-0.25 \\
-0.20 \\
-0.23 \\
-0.19 \\
-0.22 \\
-0.20 \\
-0.13 \\
-0.02 \\
-0.02 \\
-0.06 \\
-0.01 \\
\end{array}$ \\
\hline
\end{tabular}


TABLE 5-12 (continued)

\begin{tabular}{|c|c|c|c|}
\hline $\begin{array}{c}\text { Record } \\
\text { No. }\end{array}$ & $\begin{array}{c}\text { Total } \\
\text { Fluence } \\
\left(10^{21} \mathrm{n} / \mathrm{cm}^{2}\right) \\
(\mathrm{E}>0.18 \mathrm{MeV}) \\
\end{array}$ & $\begin{array}{c}\text { Irrad } \\
\text { Temp } \\
\left({ }^{0} \mathrm{C}\right)\end{array}$ & $\begin{array}{c}\text { Irrad } \\
\text { Strain } \\
\ln \left(\ell / \ell_{0}\right) \\
(10-2)\end{array}$ \\
\hline $\begin{array}{l}7515 \\
7516 \\
7517 \\
7518 \\
7519 \\
7520 \\
7521 \\
7522 \\
7523 \\
7524 \\
7525 \\
7526 \\
7527 \\
7564 \\
7565 \\
7566 \\
7567 \\
7568 \\
7569 \\
7570 \\
7571 \\
7572 \\
7573 \\
7574 \\
7575 \\
7613 \\
7614 \\
7615 \\
7616 \\
7617 \\
7618 \\
7619 \\
7620\end{array}$ & $\left.\right|^{1.7}$ & $\left.\right|^{765}$ & $\begin{array}{l}-0.02 \\
-0.03 \\
-0.03 \\
-0.05 \\
-0.02 \\
-0.03 \\
-0.01 \\
+0.01 \\
-0.04 \\
-0.04 \\
-0.04 \\
-0.06 \\
-0.03 \\
-0.07 \\
-0.06 \\
-0.06 \\
-0.02 \\
-0.04 \\
-0.02 \\
-0.05 \\
-0.02 \\
-0.06 \\
-0.07 \\
-0.11 \\
-0.08 \\
-0.07 \\
-0.09 \\
-0.10 \\
-0.08 \\
-0.09 \\
-0.07 \\
-0.08 \\
-0.08\end{array}$ \\
\hline
\end{tabular}


TABLE 5-13

DIMENSIONAL CHANGE DATA, TS-1240, RADIAL DIRECTION, AFTER IRRADIATION IN CAPSULE OG-2

\begin{tabular}{|c|c|c|c|}
\hline $\begin{array}{c}\text { Record } \\
\text { No. }\end{array}$ & $\begin{array}{c}\text { Total } \\
\text { Fluence } \\
\left(10^{21} \mathrm{n} / \mathrm{cm}^{2}\right) \\
(\mathrm{E}>0.18 \mathrm{MeV}) \\
\end{array}$ & $\begin{array}{l}\text { Irrad } \\
\text { Temp } \\
\left({ }^{\circ} \mathrm{C}\right)\end{array}$ & $\begin{array}{c}\text { Irrad } \\
\text { Strain } \\
\ln \left(\ell / \ell_{0}\right) \\
\left(10^{-2}\right)\end{array}$ \\
\hline $\begin{array}{l}6935 \\
6936 \\
6937 \\
6938 \\
6939 \\
6940 \\
6941 \\
6942 \\
7321 \\
7322 \\
7323 \\
7324 \\
7462 \\
7463 \\
7464 \\
7465 \\
7466 \\
7467 \\
7468 \\
7469 \\
7470 \\
7471 \\
7621 \\
7622 \\
7623 \\
7624\end{array}$ & $\left.\right|^{2.95}$ & $\mid$ & $\begin{array}{r}-0.48 \\
-0.45 \\
-0.45 \\
-0.45 \\
-0.47 \\
-0.49 \\
-0.53 \\
-0.46 \\
-0.05 \\
-0.04 \\
-0.05 \\
-0.04 \\
+0.02 \\
+0.02 \\
-0.05 \\
0.00 \\
-0.02 \\
-0.02 \\
+0.01 \\
-0.02 \\
0.00 \\
-0.01 \\
-0.13 \\
-0.07 \\
-0.11 \\
-0.07 \\
\end{array}$ \\
\hline
\end{tabular}


TABLE 5-14

DIMENSIONAL CHANGE DATA, $\mathrm{P}_{3}$ JHAN, AXIAL DIRECTION, AFTER IRRADIATION IN CAPSULE OG-1

\begin{tabular}{|c|c|c|c|}
\hline $\begin{array}{c}\text { Record } \\
\text { No. }\end{array}$ & $\begin{array}{c}\text { Total } \\
\text { Fluence } \\
\left(10^{21} \mathrm{n} / \mathrm{cm}^{2}\right) \\
(\mathrm{E}>0.18 \mathrm{MeV})\end{array}$ & $\begin{array}{l}\text { Irrad } \\
\text { Temp } \\
\left({ }^{\circ} \mathrm{C}\right)\end{array}$ & $\begin{array}{l}\text { Irrad } \\
\text { Strain } \\
\ln \left(\ell / \ell_{0}\right) \\
(10-2)\end{array}$ \\
\hline 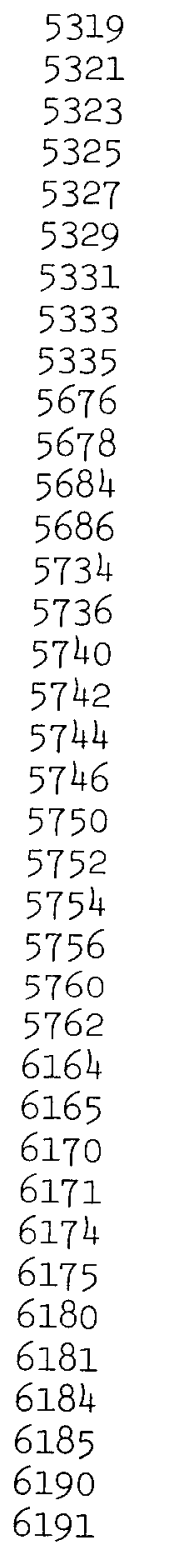 & ${ }_{3.0}^{1.9}$ & $\begin{array}{l}\mid \\
1380 \\
1335 \\
1025 \\
\mid\end{array}$ & $\begin{array}{l}-1.53 \\
-2.04 \\
-2.02 \\
-1.48 \\
-1.99 \\
-2.00 \\
-1.44 \\
-1.82 \\
-2.11 \\
-3.96 \\
-4.00 \\
-4.19 \\
-4.06 \\
-2.42 \\
-1.97 \\
-2.31 \\
-1.83 \\
-2.17 \\
-1.87 \\
-2.13 \\
-1.83 \\
-2.03 \\
-1.77 \\
-2.21 \\
-1.82 \\
-0.26 \\
-0.23 \\
-0.37 \\
-0.24 \\
-0.35 \\
-0.23 \\
-0.28 \\
-0.28 \\
-0.28 \\
-0.28 \\
-0.30 \\
-0.28\end{array}$ \\
\hline
\end{tabular}


TABLE 5-14 (continued)

\begin{tabular}{|c|c|c|c|}
\hline $\begin{array}{c}\text { Record } \\
\text { No. }\end{array}$ & $\begin{array}{c}\text { Total } \\
\text { Fluence } \\
\left(10^{21} \mathrm{n} / \mathrm{cm}^{2}\right) \\
(\mathrm{E}>0.18 \mathrm{MeV})_{\mathrm{HTGR}}\end{array}$ & $\begin{array}{l}\text { Irrad } \\
\text { Temp } \\
\left({ }^{\circ} \mathrm{C}\right)\end{array}$ & $\begin{array}{c}\text { Irrad } \\
\text { strain } \\
\ln \left(\ell / \ell_{O}\right) \\
(10-2)\end{array}$ \\
\hline $\begin{array}{l}6332 \\
6334 \\
6336 \\
6338 \\
6342 \\
6344 \\
6347 \\
6349\end{array}$ & $\sum_{1.4}^{1.4}$ & $\downarrow_{605}$ & $\begin{array}{l}-0.23 \\
-0.16 \\
-0.20 \\
-0.19 \\
-0.20 \\
-0.22 \\
-0.17 \\
-0.21\end{array}$ \\
\hline
\end{tabular}


TABLE 5-15

DIMENSIONAL CHANGE DATA, $P_{3}$ JHAN, AXIAL DIRECTION, AFTER IRRADIATION IN CAPSULE OG-2

\begin{tabular}{|c|c|c|c|c|c|c|c|c|}
\hline $\begin{array}{c}\text { Record } \\
\text { No. }\end{array}$ & $\begin{array}{c}\text { Previous } \\
\text { Record } \\
\text { No. }\end{array}$ & $\begin{array}{c}\text { Previous } \\
\text { Fluence } \\
\left(10^{21} \mathrm{n} / \mathrm{cm}^{2}\right) \\
(\mathrm{E}>0.18 \mathrm{MeV})_{\mathrm{HTGR}}\end{array}$ & $\begin{array}{c}\text { Previous } \\
\text { Temp } \\
\left({ }^{\circ} \mathrm{C}\right)\end{array}$ & $\begin{array}{c}\text { Fluence } \\
\text { This Irrad } \\
\left(10^{21} \mathrm{n} / \mathrm{cm}^{2}\right) \\
(\mathrm{E}>0.18 \mathrm{MeV})_{\text {HTGR }}\end{array}$ & $\begin{array}{l}\text { Temp } \\
\text { This } \\
\text { Irrad } \\
\left({ }^{\circ} \mathrm{C}\right)\end{array}$ & $\begin{array}{c}\text { Total } \\
\text { Fluence } \\
\left(10^{21} \mathrm{n} / \mathrm{cm}^{2}\right) \\
(E>0.18 \mathrm{MeV})_{\text {HTGR }}\end{array}$ & $\begin{array}{l}\text { Irrad } \\
\text { Temp } \\
\left({ }^{\circ} \mathrm{C}\right)\end{array}$ & $\begin{array}{l}\text { Irrad } \\
\text { Strain } \\
\ln (\ell / \ell 0) \\
\left(10^{-2}\right)\end{array}$ \\
\hline 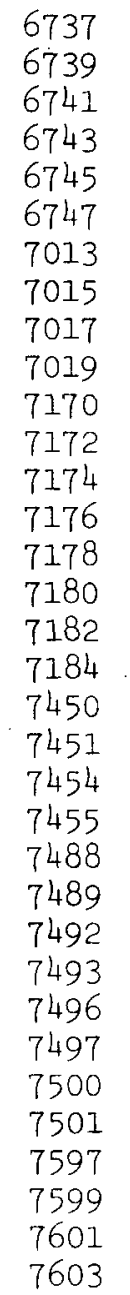 & $\begin{array}{l}5319 \\
5321 \\
5323 \\
5325 \\
5327 \\
5329 \\
5676 \\
5678 \\
5684 \\
5686 \\
5734 \\
5736 \\
5740 \\
5742 \\
5744 \\
5746 \\
5750 \\
5752 \\
6164 \\
6165 \\
6170 \\
6171 \\
6184 \\
6185 \\
6190 \\
6191 \\
6174 \\
6175 \\
6180 \\
6181 \\
6332 \\
6334 \\
6336 \\
6338\end{array}$ & $\begin{array}{l}3.0 \\
3.0 \\
3.9 \\
3.0 \\
31\end{array}$ & \begin{tabular}{|}
980 \\
980 \\
1335 \\
1335 \\
1025 \\
1 \\
1025 \\
770 \\
1
\end{tabular} & $\begin{array}{l}1 \\
2.7 \\
2.9 \\
2.7 \\
1 \\
1.7 \\
1.7 \\
1.15 \\
1 \\
15 \\
15 \\
15\end{array}$ & $\begin{array}{l}\left.\right|_{960} \\
820 \\
820 \\
765 \\
1 \\
765 \\
590 \\
1\end{array}$ & $\begin{array}{l}\mid \\
5.7 \\
3.6 \\
1.6 \\
1.6 \\
2.55 \\
1 \\
1.55\end{array}$ & $\left.\right|_{985} ^{985}$ & $\begin{array}{l}-3.36 \\
-3.59 \\
-3.54 \\
-3.52 \\
-3.74 \\
-3.44 \\
-3.67 \\
-3.60 \\
-4.12 \\
-3.68 \\
-3.88 \\
-3.68 \\
-3.69 \\
-3.39 \\
-3.46 \\
-3.64 \\
-3.46 \\
-3.72 \\
-0.98 \\
-0.88 \\
-1.07 \\
-0.90 \\
-0.88 \\
-0.80 \\
-0.97 \\
-0.89 \\
-0.95 \\
-0.77 \\
-0.90 \\
-0.78 \\
-0.50 \\
-0.40 \\
-0.49 \\
-0.38\end{array}$ \\
\hline
\end{tabular}


TABLE 5-16

DIMENSIONAL CHANGE DATA, $\mathrm{P}_{3}$ JHAN, RADIAL DIRECTION, AFTER IRRADIATION IN CAPSULE OG-1

\begin{tabular}{|c|c|c|c|}
\hline $\begin{array}{c}\text { Record } \\
\text { No. }\end{array}$ & $\begin{array}{c}\text { Total } \\
\text { Fluence } \\
\left(10^{21} \mathrm{n} / \mathrm{cm}^{2}\right) \\
(\mathrm{E}>0.18 \mathrm{MeV})_{\text {HTGR }}\end{array}$ & $\begin{array}{r}\text { Irrad } \\
\text { Temp } \\
\left({ }^{\circ} \mathrm{C}\right)\end{array}$ & $\begin{array}{l}\text { Irrad } \\
\text { Strain } \\
\ln \left(\ell / \ell_{0}\right) \\
(10-2)\end{array}$ \\
\hline $\begin{array}{l}5320 \\
5322 \\
5324 \\
5326 \\
5328 \\
5330 \\
5332 \\
5334 \\
5336 \\
5677 \\
5679 \\
5685 \\
5687 \\
5735 \\
5737 \\
5741 \\
5743 \\
5745 \\
5747 \\
5751 \\
5753 \\
5757 \\
5761 \\
5763 \\
6166 \\
6167 \\
6172 \\
6173 \\
6176 \\
6177 \\
6182 \\
6183 \\
6186 \\
6187 \\
6192 \\
6193 \\
6333 \\
6335 \\
6337 \\
6339\end{array}$ & $\begin{array}{l}\prod^{3.0} \\
3.0 \\
3.2 \\
3.0 \\
1 \\
1\end{array}$ & \begin{tabular}{|}
980 \\
1380 \\
1335 \\
1025 \\
1
\end{tabular} & $\begin{array}{l}-0.69 \\
-0.76 \\
-0.58 \\
-0.73 \\
-0.87 \\
-0.83 \\
-0.66 \\
-0.78 \\
-0.73 \\
-1.66 \\
-1.44 \\
-1.65 \\
-1.68 \\
-0.81 \\
-0.90 \\
-0.87 \\
-0.84 \\
-0.81 \\
-0.86 \\
-0.71 \\
-0.67 \\
-0.70 \\
-0.81 \\
-0.83 \\
-0.11 \\
-0.08 \\
-0.17 \\
-0.13 \\
-0.18 \\
-0.01 \\
-0.22 \\
-0.11 \\
-0.10 \\
-0.09 \\
-0.16 \\
-0.11 \\
-0.15 \\
-0.10 \\
-0.17 \\
-0.06\end{array}$ \\
\hline
\end{tabular}


TABLE 5-16 (continued)

\begin{tabular}{c|c|c|c}
\hline & $\begin{array}{c}\text { Total } \\
\text { Fluence } \\
\left(10^{21} \mathrm{n} / \mathrm{cm}^{2}\right)\end{array}$ & $\begin{array}{c}\text { Irrad } \\
\text { Temp } \\
\left({ }^{\circ} \mathrm{C}\right)\end{array}$ & $\begin{array}{c}\text { Irrad } \\
\text { Strain } \\
\ln (\ell / \ell \\
\left(10^{-2}\right)\end{array}$ \\
\hline No. & 1.4 & 605 & -0.18 \\
6343 & 1 & 1 & -0.06 \\
6345 & 1.4 & 605 & -0.15 \\
6348 & $18 \mathrm{MeV})$ & -0.13 \\
6350 & & &
\end{tabular}


TABLE 5-17

DIMENSIONAL CHANGE DATA, $P_{3}$ JHAN, RADIAL DIRECTION, AFTER IRRADIATION IN CAPSULE OG-2

\begin{tabular}{|c|c|c|c|c|c|c|c|c|}
\hline $\begin{array}{c}\text { Record } \\
\text { No. }\end{array}$ & $\begin{array}{c}\text { Previous } \\
\text { Record } \\
\text { No. }\end{array}$ & $\begin{array}{c}\text { Previous } \\
\text { Fluence } \\
\left(10^{21} \mathrm{n} / \mathrm{cm}^{2}\right) \\
(E>0.18 \mathrm{MeV})_{\text {HTGR }}\end{array}$ & $\begin{array}{l}\text { Previous } \\
\text { Temp } \\
\left({ }^{\circ} \mathrm{C}\right)\end{array}$ & $\begin{array}{c}\text { Fluence } \\
\text { This Irrad } \\
\left(10^{21} \mathrm{n} / \mathrm{cm}^{2}\right) \\
(\mathrm{E}>0.18 \mathrm{MeV})_{\text {HTGR }}\end{array}$ & $\begin{array}{l}\text { Temp } \\
\text { This } \\
\text { Irrad } \\
\left({ }^{\circ} \mathrm{C}\right)\end{array}$ & $\begin{array}{c}\text { Total } \\
\text { Fluence } \\
\left(10^{21} \mathrm{n} / \mathrm{cm}^{2}\right) \\
(\mathrm{E}>0.18 \mathrm{MeV})_{\text {HTGR }}\end{array}$ & $\begin{array}{l}\text { Irrad } \\
\text { Temp } \\
\left({ }^{\circ} \mathrm{C}\right)\end{array}$ & $\begin{array}{c}\text { Irrad } \\
\text { Strain } \\
\ln \left(\ell / \ell_{0}\right) \\
(10-2)\end{array}$ \\
\hline 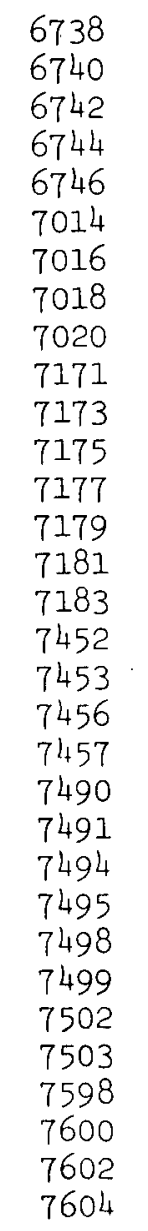 & $\begin{array}{l}5320 \\
5322 \\
5324 \\
5326 \\
5328 \\
5677 \\
5679 \\
5685 \\
5687 \\
5735 \\
5737 \\
5741 \\
5743 \\
5745 \\
5747 \\
5751 \\
6166 \\
6167 \\
6172 \\
6173 \\
6186 \\
6187 \\
6192 \\
6193 \\
6176 \\
6177 \\
6182 \\
6183 \\
6333 \\
6335 \\
6337 \\
6339\end{array}$ & $\begin{array}{l}3.0 \\
3.0 \\
3.2 \\
3.0 \\
3.0 \\
1.9 \\
1.9 \\
1.4 \\
1 \\
1\end{array}$ & \begin{tabular}{|}
980 \\
980 \\
1335 \\
1335 \\
1025 \\
1025 \\
770 \\
$\mid$
\end{tabular} & $\begin{array}{l}\left.\right|^{2.7} \\
2.7 \\
2.9 \\
2.7 \\
2.7 \\
1.7 \\
1.7 \\
15 \\
15\end{array}$ & \begin{tabular}{|}
990 \\
990 \\
1380 \\
1380 \\
960 \\
1 \\
960 \\
820 \\
1 \\
820 \\
765 \\
1
\end{tabular} & $\begin{array}{l}i^{1} \\
6.7 \\
6.1 \\
5.7 \\
i^{7} \\
5.7 \\
3.6 \\
1\end{array}$ & $\begin{array}{c}1 \\
985 \\
1355 \\
1 \\
1355 \\
995 \\
1\end{array}$ & $\begin{array}{l}+0.19 \\
+0.82 \\
+1.64 \\
+0.06 \\
+0.71 \\
+3.43 \\
2.66 \\
3.46 \\
2.02 \\
+0.32 \\
-0.91 \\
+0.10 \\
-0.60 \\
+0.72 \\
-0.67 \\
+0.55 \\
-0.43 \\
-0.36 \\
-0.43 \\
-0.35 \\
-0.41 \\
-0.35 \\
-0.46 \\
-0.36 \\
-0.37 \\
-0.32 \\
-0.41 \\
-0.32 \\
-0.37 \\
-0.33 \\
-0.08 \\
-0.27\end{array}$ \\
\hline
\end{tabular}


demonstrated more moderate dimensional changes than observed in earlier irradiations. Tables 5-18 and 5-19 list the $O G-1$ and $O G-2$ data in terms of HTGR Eiluence $(\mathrm{E}>0.18 \mathrm{MeV})_{\mathrm{HTGR}}$ for $434 \mathrm{H}-327$ specimens.

\subsection{MISCELLANEOUS GRAPHITES}

Impregnated H-451 graphites were irradiated in capsule OG-2. The data obtained are presented in Table 5-20. The H-451C material, which was used as web material during cure-in-place studies, exhibited the same dimensional change as standard H-451 irradiated under the same conditions.

Other miscellaneous graphites were also irradiated in capsule OG-2. These dat:a are presented in Tables 5-21, 5-22, and 5-23. 
TABLE 5-18

DIMENSIONAL CHANGE DATA, H-327, AXIAL DIRECTION, AFTER IRRADLATION IN CAPSULES OG-1 AND OG-2

\begin{tabular}{|c|c|c|c|c|c|c|c|c|c|}
\hline $\begin{array}{c}\text { Record } \\
\text { No. }\end{array}$ & $\begin{array}{l}\text { Previous } \\
\text { Record } \\
\text { No. }\end{array}$ & $\begin{array}{c}\text { Previous } \\
\text { Fluence } \\
\left(10^{21} \mathrm{n} / \mathrm{cm}^{2}\right) \\
(E>0.18 \mathrm{MeV}) \text { HTGR }\end{array}$ & $\begin{array}{l}\text { Previous } \\
\text { Temp. } \\
\left({ }^{\circ} \mathrm{C}\right)\end{array}$ & $\begin{array}{c}\text { Fluence } \\
\text { This Irrad } \\
\left(10^{21} \mathrm{n} / \mathrm{cm}^{2}\right)^{\circ} \\
(\mathrm{E}>0.18 \mathrm{MeV})_{\mathrm{HTGR}}\end{array}$ & $\begin{array}{l}\text { Temp. } \\
\text { This } \\
\text { Irrad. } \\
\left({ }^{\circ} \mathrm{C}\right)\end{array}$ & $\begin{array}{c}\text { Total Fluence } \\
\left(10^{21} \mathrm{n} / \mathrm{cm}^{2}\right) \\
(E>0.18 \mathrm{MeV})_{\mathrm{HTGR}}\end{array}$ & $\begin{array}{l}\text { Irrad. } \\
\text { Temp. } \\
\left({ }^{\circ} \mathrm{C}\right)\end{array}$ & $\begin{array}{c}\text { Irrad. } \\
\text { Strain, } \\
\ln \left(\ell / \ell_{0}\right) \\
\left(10^{-2}\right)\end{array}$ & $\begin{array}{c}\text { Dimen. } \\
\text { Change, } \\
\Delta \ell / \ell \\
(\%)\end{array}$ \\
\hline $\begin{array}{l}5005 \\
5009 \\
5013 \\
5017 \\
5021 \\
5025 \\
5029 \\
5033 \\
5037 \\
5041 \\
5114 \\
5115 \\
5118 \\
5119 \\
5124 \\
5125 \\
5128 \\
5129 \\
5134 \\
5135 \\
5147 \\
5151 \\
5155 \\
5159 \\
5163 \\
5167 \\
5171 \\
5175 \\
5179 \\
5182 \\
5185 \\
5186 \\
5195 \\
5196 \\
5205 \\
5206 \\
5215 \\
5216 \\
5241 \\
5242 \\
5251 \\
5252 \\
5257 \\
5258 \\
5261 \\
5262 \\
5263 \\
5264 \\
5267 \\
5268\end{array}$ & . & . & . & . & 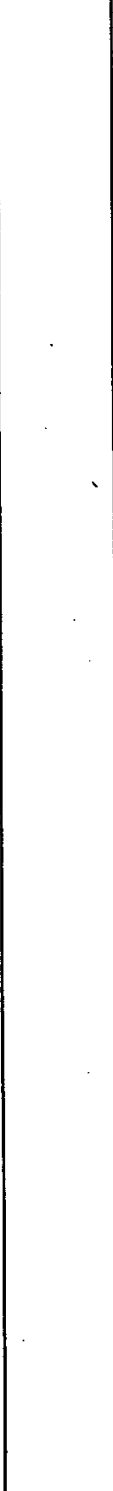 & 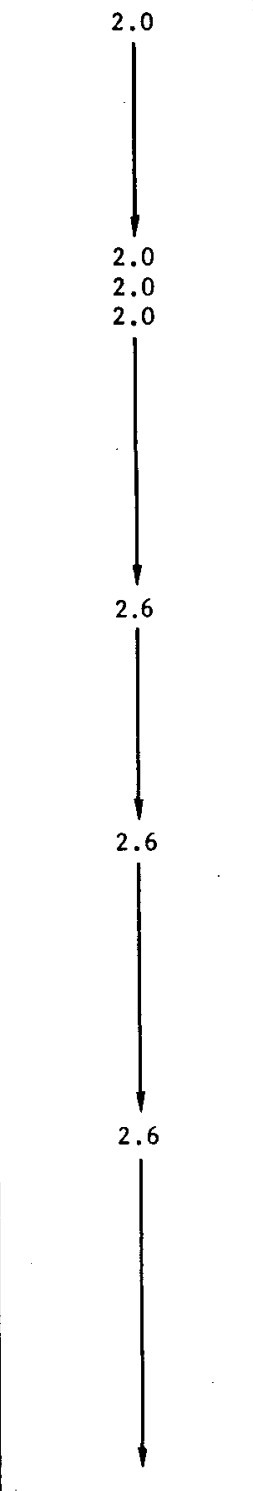 & 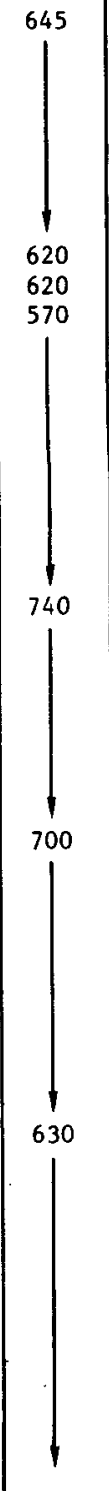 & $\begin{array}{l}-0.15 \\
-0.16 \\
-0.17 \\
-0.14 \\
-0.11 \\
-0.13 \\
-0.11 \\
-0.15 \\
-0.20 \\
-0.18 \\
-0.14 \\
-0.16 \\
-0.16 \\
-0.20 \\
-0.15 \\
-0.21 \\
-0.14 \\
-0.18 \\
-0.14 \\
-0.20 \\
-0.25 \\
-0.24 \\
-0.17 \\
-0.24 \\
-0.26 \\
-0.20 \\
-0.23 \\
-0.17 \\
-0.24 \\
-0.28 \\
-0.24 \\
-0.17 \\
-0.21 \\
-0.10 \\
-0.25 \\
-0.21 \\
-0.22 \\
-0.23 \\
-0.28 \\
-0.33 \\
-0.28 \\
-0.31 \\
-0.24 \\
-0.34 \\
-0.32 \\
-0.27 \\
-0.34 \\
-0.33 \\
-0.25 \\
-0.21\end{array}$ & . \\
\hline
\end{tabular}


TABLE 5-18 (Continued)

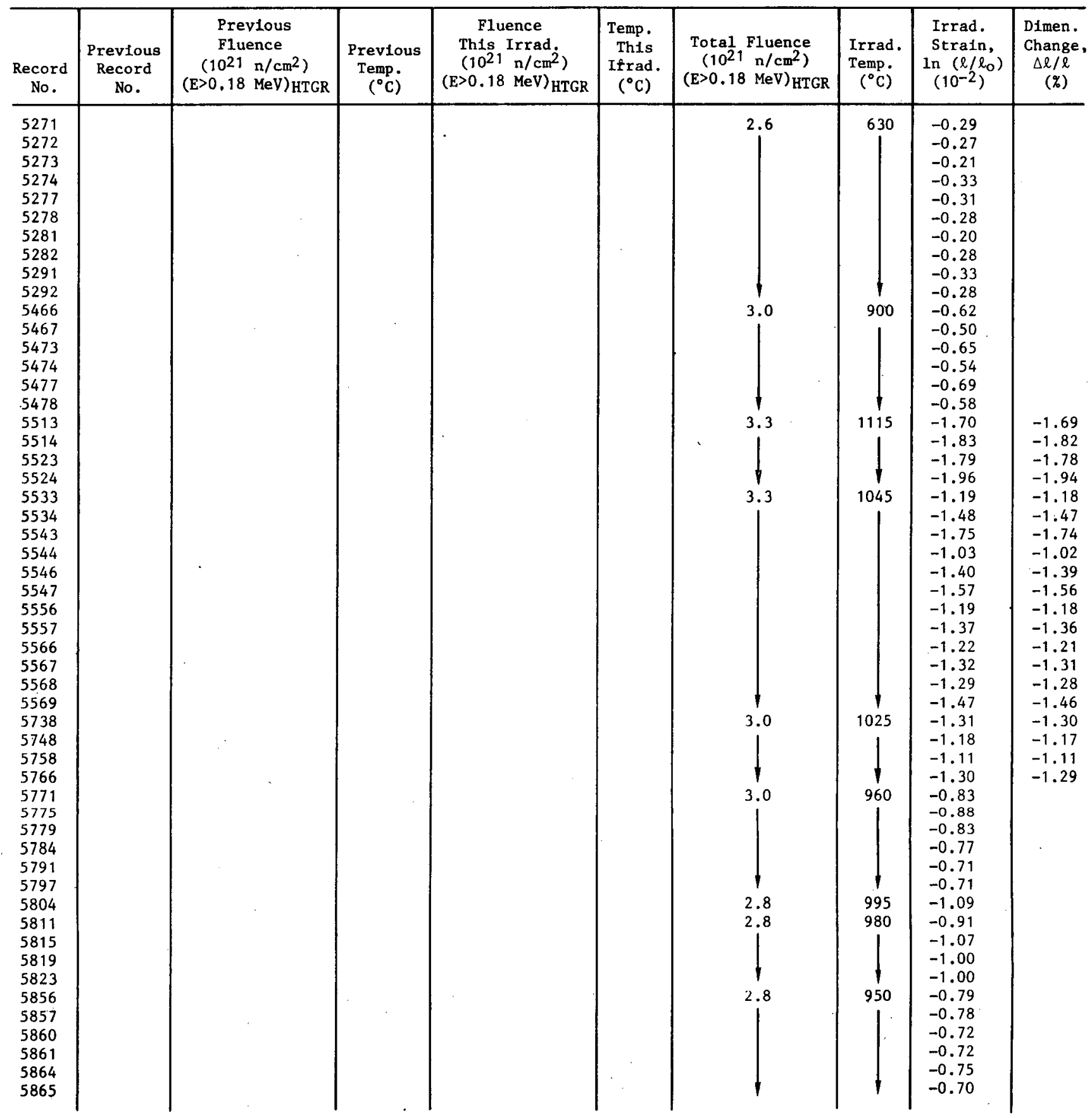


TABLE 5-18 (continued)

\begin{tabular}{|c|c|c|c|c|c|c|c|c|c|}
\hline $\begin{array}{c}\text { Record } \\
\text { No. }\end{array}$ & $\begin{array}{c}\text { Previous } \\
\text { Record } \\
\text { No. }\end{array}$ & $\begin{array}{c}\text { Prevlous } \\
\text { Fluence } \\
\left(10^{21} \mathrm{n} / \mathrm{cm}^{2}\right) \\
(E>0.18 \mathrm{MeV})_{\mathrm{HTGR}}\end{array}$ & $\begin{array}{c}\text { Previous } \\
\text { Temp. } \\
\left({ }^{\circ} \mathrm{C}\right)\end{array}$ & $\begin{array}{c}\text { Fluence } \\
\text { Th1s Irrad. } \\
\left(10^{21} \mathrm{n} / \mathrm{cm}^{2}\right) \\
(E>0.18 \mathrm{MeV})_{\text {HTGR }}\end{array}$ & $\begin{array}{l}\text { Temp. } \\
\text { This } \\
\text { Irrad. } \\
\left({ }^{\circ} \mathrm{C}\right)\end{array}$ & $\begin{array}{l}\text { Total Fluence } \\
\quad\left(10^{21} \mathrm{n} / \mathrm{cm}^{2}\right) \\
(E>0.18 \mathrm{MeV})_{\mathrm{HTGR}}\end{array}$ & $\begin{array}{l}\text { Irrad } \\
\text { Temp } \\
\left({ }^{\circ} \mathrm{C}\right)\end{array}$ & $\begin{array}{l}\text { Irrad. } \\
\text { Strain, } \\
\text { ln }\left(\ell / \ell_{0}\right) \\
\left(10^{-2}\right)\end{array}$ & $\begin{array}{l}\text { Dimen. } \\
\text { Change, } \\
\Delta \ell / \ell \\
(\%)\end{array}$ \\
\hline $\begin{array}{l}5907 \\
5911 \\
5915 \\
5919 \\
5923 \\
5927 \\
5954 \\
5955 \\
5964 \\
5965 \\
5974 \\
5975 \\
5996 \\
5997 \\
6006 \\
6007 \\
6008 \\
6009 \\
6018 \\
6019 \\
6024 \\
6025 \\
6028 \\
6029 \\
6030 \\
6031 \\
6034 \\
6035 \\
6038 \\
6039 \\
6092 \\
6093 \\
6102 \\
6103 \\
6112 \\
6113 \\
6122 \\
6123 \\
6148 \\
6149 \\
6158 \\
6159 \\
6168 \\
6169 \\
6178 \\
6179 \\
6188 \\
6189 \\
6198 \\
6199\end{array}$ & . & , & & $\checkmark$ & . & $\left.\right|_{13} ^{2.3}$ & 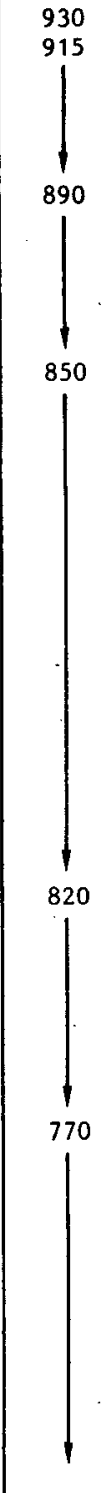 & $\begin{array}{l}-0.60 \\
-0.49 \\
-0.52 \\
-0.56 \\
-0.52 \\
-0.51 \\
-0.42 \\
-0.34 \\
-0.39 \\
-0.30 \\
-0.43 \\
-0.31 \\
-0.33 \\
-0.25 \\
-0.29 \\
-0.20 \\
-0.31 \\
-0.20 \\
-0.27 \\
-0.16 \\
-0.32 \\
-0.27 \\
-0.29 \\
-0.20 \\
-0.26 \\
-0.28 \\
-0.27 \\
-0.30 \\
-0.64 \\
-0.20 \\
-0.17 \\
-0.11 \\
-0.15 \\
-0.11 \\
-0.15 \\
-0.12 \\
-0.05 \\
-0.11 \\
-0.15 \\
-0.18 \\
-0.11 \\
-0.07 \\
-0.18 \\
-0.09 \\
-0.12 \\
-0.15 \\
-0.13 \\
-0.13 \\
-0.08\end{array}$ & \\
\hline
\end{tabular}


TABLE 5-18 (continued)

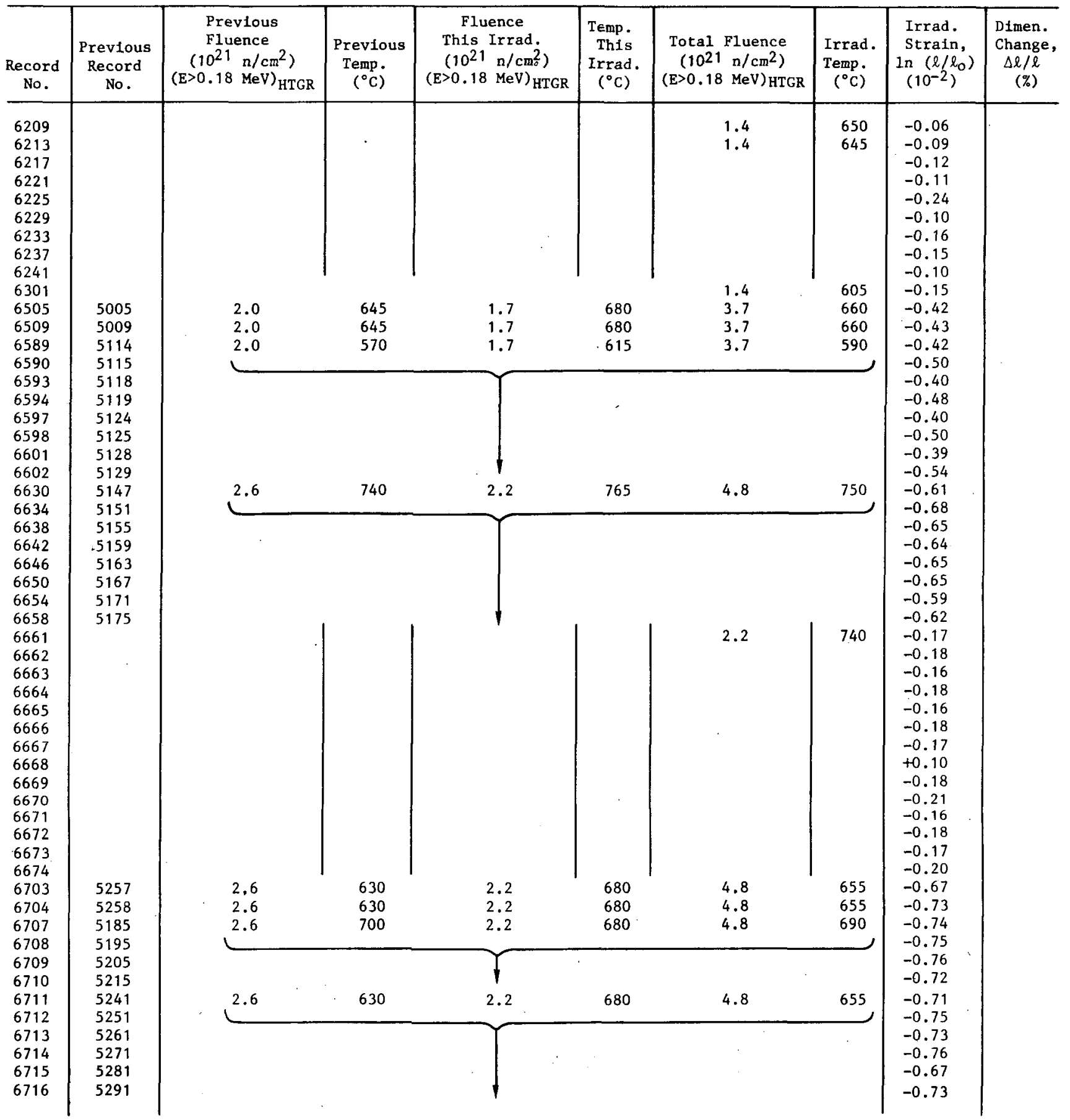


TABLE 5-18 (continued)

\begin{tabular}{|c|c|c|c|c|c|c|c|c|c|}
\hline $\begin{array}{c}\text { Record } \\
\text { No. }\end{array}$ & $\begin{array}{c}\text { Prevlous } \\
\text { Record } \\
\text { No. }\end{array}$ & $\begin{array}{c}\text { Previous } \\
\text { Fluence } \\
\left(10^{21} \mathrm{n} / \mathrm{cm}^{2}\right) \\
(\mathrm{E}>0.18 \mathrm{MeV})_{\text {HTGR }}\end{array}$ & $\begin{array}{l}\text { Previous } \\
\text { Temp. } \\
\left({ }^{\circ} \mathrm{C}\right)\end{array}$ & $\begin{array}{l}\text { Fluence } \\
\text { Th1s Irrad. } \\
\left(10^{21} \mathrm{n} / \mathrm{cm}^{2}\right) \\
(E>0.18 \mathrm{MeV})_{\text {HTGR }}\end{array}$ & $\begin{array}{l}\text { Temp. } \\
\text { This } \\
\text { Irrad. } \\
\left({ }^{\circ} \mathrm{C}\right)\end{array}$ & $\begin{array}{c}\text { Total Fluence } \\
\left(10^{21} \mathrm{n} / \mathrm{cm}^{2}\right) \\
(\mathrm{E}>0.18 \mathrm{MeV})_{\mathrm{HTGR}}\end{array}$ & $\begin{array}{l}\text { Irrad. } \\
\text { Temp. } \\
\left({ }^{\circ} \mathrm{C}\right)\end{array}$ & $\begin{array}{l}\text { Irrad. } \\
\text { Strain, } \\
\text { 1n }\left(\ell / \ell_{0}\right) \\
\left(10^{-2}\right)\end{array}$ & $\begin{array}{c}\text { Dimen. } \\
\text { Change, } \\
\Delta \ell / \ell \\
(\%)\end{array}$ \\
\hline $\begin{array}{l}6717 \\
6718 \\
6719 \\
6720 \\
6767\end{array}$ & 5466 & 3.0 & 900 & 2.7 & 990 & $i_{5.7}^{2.2}$ & $\left.\right|_{940} ^{680}$ & $\begin{array}{l}-0.20 \\
-0.22 \\
-0.22 \\
-0.23 \\
-2.18\end{array}$ & -2.15 \\
\hline 6768 & 5467 & & & & & & & -2.39 & -2.36 \\
\hline $\begin{array}{l}6769 \\
6773\end{array}$ & $\begin{array}{l}5473 \\
5474\end{array}$ & & & & & & & $\begin{array}{l}-2.51 \\
-2.37\end{array}$ & $\begin{array}{l}-2.48 \\
-2.34\end{array}$ \\
\hline 6774 & 5477 & & & & & & & -2.48 & -2.45 \\
\hline 6775 & 5478 & & & 1 & & & & -2.33 & -2.30 \\
\hline 6943 & 5513 & 3.3 & 1115 & 2.95 & 1200 & 6.25 & 1155 & -3.98 & -3.90 \\
\hline 6944 & 5523 & 3.3 & 1115 & 2.95 & 1200 & 6.25 & 1155 & -4.33 & -4.24 \\
\hline 6961 & 5533 & 3.3 & 1045 & 2.95 & 1105 & 6.25 & 1075 & -3.18 & -3.13 \\
\hline 6962 & 5543 & & & & & & & -4.33 & -4.24 \\
\hline 6963 & 5546 & & & & & & & -3.25 & -3.19 \\
\hline 6964 & 5556 & & & & & & & -3.38 & -3.32 \\
\hline 6965 & 5566 & & & & & & & -2.61 & -2.57 \\
\hline 6966 & 5568 & & & & & & & -3.49 & -3.43 \\
\hline 7119 & 5738 & 3.0 & 1025 & 2.7 & 1035 & 5.7 & 1030 & -3.87 & -3.80 \\
\hline 7120 & 5748 & & & & & & & -3.43 & -3.37 \\
\hline 7121 & 5758 & & & & & & & -3.78 & -3.71 \\
\hline 7122 & 5766 & & & 1 & & & & -3.85 & -3.78 \\
\hline 7123 & & & & & & 2.7 & 1035 & -1.48 & -1.47 \\
\hline 7124 & & & & & & 1 & & -1.13 & -1.13 \\
\hline 7125 & & & & & & & & -1.28 & -1.27 \\
\hline 7126 & & & & & & 1 & 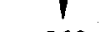 & -0.99 & \\
\hline 7150 & 5771 & 3.0 & 960 & 2.7 & 960 & 5.7 & 960 & -2.67 & -2.63 \\
\hline 7151 & 5775 & 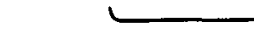 & & & & & & -2.42 & -2.39 \\
\hline 7152 & 5779 & & . & & & & & -2.71 & -2.67 \\
\hline 7153 & 5784 & & & & & & & -2.16 & -2.14 \\
\hline 7154 & 5791 & & & & & & & -2.39 & -2.36 \\
\hline 7155 & 5797 & & & & & & & -2.04 & -2.01 \\
\hline 7156 & & & & & & 2.7 & 960 & -1.02 & \\
\hline 7157 & & & & & & 1 & & -0.71 & \\
\hline 7158 & & & & & & & & -0.98 & \\
\hline 7159 & & & & & & & & -0.81 & \\
\hline $\begin{array}{l}7160 \\
7161\end{array}$ & & & & & & 1 & 1 & $\begin{array}{l}-1.06 \\
-0.78\end{array}$ & \\
\hline 7216 & 5804 & 2.8 & 995 & 2.5 & 1000 & 5.3 & 995 & -3.20 & -3.15 \\
\hline 7220 & 5811 & 2.8 & 995 & 2.5 & 980 & 5.3 & 985 & -2.79 & -2.75 \\
\hline 7224 & 5815 & 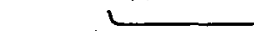 & & & & & & -3.06 & -3.01 \\
\hline 7228 & 5819 & & & & & & & -2.93 & -2.89 \\
\hline 7232 & 5823 & & & 1 & & & & -3.10 & -3.05 \\
\hline 7304 & 5857 & 2.8 & 950 & 2.5 & 920 & 5.3 & 935 & -2.18 & -2.15 \\
\hline 7402 & 6008 & 2.3 & 850 & 2.15 & 850 & 4.45 & 850 & -0.98 & \\
\hline 7403 & 6009 & $\smile$ & & & & & & -0.75 & \\
\hline 7404 & 6018 & & & & & & & -0.93 & \\
\hline 7405 & 6019 & & & & & & & -0.71 & \\
\hline 7406 & 6028 & & & & & & & -0.97 & \\
\hline 7407 & 6029 & & & & & & & -0.71 & \\
\hline 7408 & 6038 & & & & & & & -1.37 & -1.36 \\
\hline 7409 & 6039 & & & 1 & & & & -0.75 & \\
\hline
\end{tabular}


TABLE 5-18 (continued)

\begin{tabular}{|c|c|c|c|c|c|c|c|c|c|}
\hline $\begin{array}{c}\text { Record } \\
\text { No. }\end{array}$ & $\begin{array}{c}\text { Previous } \\
\text { Record } \\
\text { No. }\end{array}$ & $\begin{array}{c}\text { Previous } \\
\text { Fluence } \\
\left(10^{21} \mathrm{n} / \mathrm{cm}^{2}\right) \\
(E>0.18 \mathrm{MeV})_{\text {HTGR }}\end{array}$ & $\begin{array}{l}\text { Previous } \\
\text { Temp. } \\
\left({ }^{\circ} \mathrm{C}\right)\end{array}$ & $\begin{array}{c}\text { Fluence } \\
\text { This Irrad } \\
\left(10^{21} \mathrm{n} / \mathrm{cm}^{2}\right) \\
(\mathrm{E}>0.18 \mathrm{MeV})_{\mathrm{HTGR}}\end{array}$ & $\begin{array}{l}\text { Temp. } \\
\text { This } \\
\text { Irrad. } \\
\left({ }^{\circ} \mathrm{C}\right)\end{array}$ & $\begin{array}{c}\text { Total Fluence } \\
\left(10^{21} \mathrm{n} / \mathrm{cm}^{2}\right) \\
(E>0.18 \mathrm{MeV})_{\text {HTGR }}\end{array}$ & $\begin{array}{l}\text { Irrad. } \\
\text { Temp. } \\
\left({ }^{\circ} \mathrm{C}\right)\end{array}$ & $\begin{array}{c}\text { Irrad. } \\
\text { Strain, } \\
\ln \left(\ell / \ell_{0}\right) \\
\left(10^{-2}\right)\end{array}$ & $\begin{array}{c}\text { D1men. } \\
\text { Change, } \\
\Delta \ell / \ell \\
(\%)\end{array}$ \\
\hline $\begin{array}{l}7458 \\
7459 \\
7460 \\
7461 \\
7504 \\
7505 \\
7506 \\
7507 \\
7508 \\
7509 \\
7529 \\
7533 \\
7537 \\
7541 \\
7545\end{array}$ & $\begin{array}{l}6092 \\
6102 \\
6112 \\
6122 \\
6148 \\
6158 \\
6168 \\
6178 \\
6188 \\
6198 \\
6209 \\
6213 \\
6217 \\
6221 \\
6225\end{array}$ & $\begin{array}{l}1.4 \\
1.4 \\
\end{array}$ & $\begin{array}{r}820 \\
770 \\
\end{array}$ & $\begin{array}{l}1 \\
1.15 \\
1.15\end{array}$ & $\begin{array}{l}765 \\
= \\
645 \\
635\end{array}$ & $\begin{array}{l}2.55 \\
2.55\end{array}$ & & $\begin{array}{l}-0.49 \\
-0.44 \\
-0.54 \\
-0.49 \\
-0.43 \\
-0.35 \\
-0.41 \\
-0.42 \\
-0.41 \\
-0.38 \\
-0.24 \\
-0.28 \\
-0.27 \\
-0.23 \\
-0.28\end{array}$ & \\
\hline
\end{tabular}


TABLE 5-19

DIMENSIONAL CHANGE DATA, H-327, RADIAL DIRECTION, AFTER IRRADIATION IN CAPSULES OG-1 AND OG-2

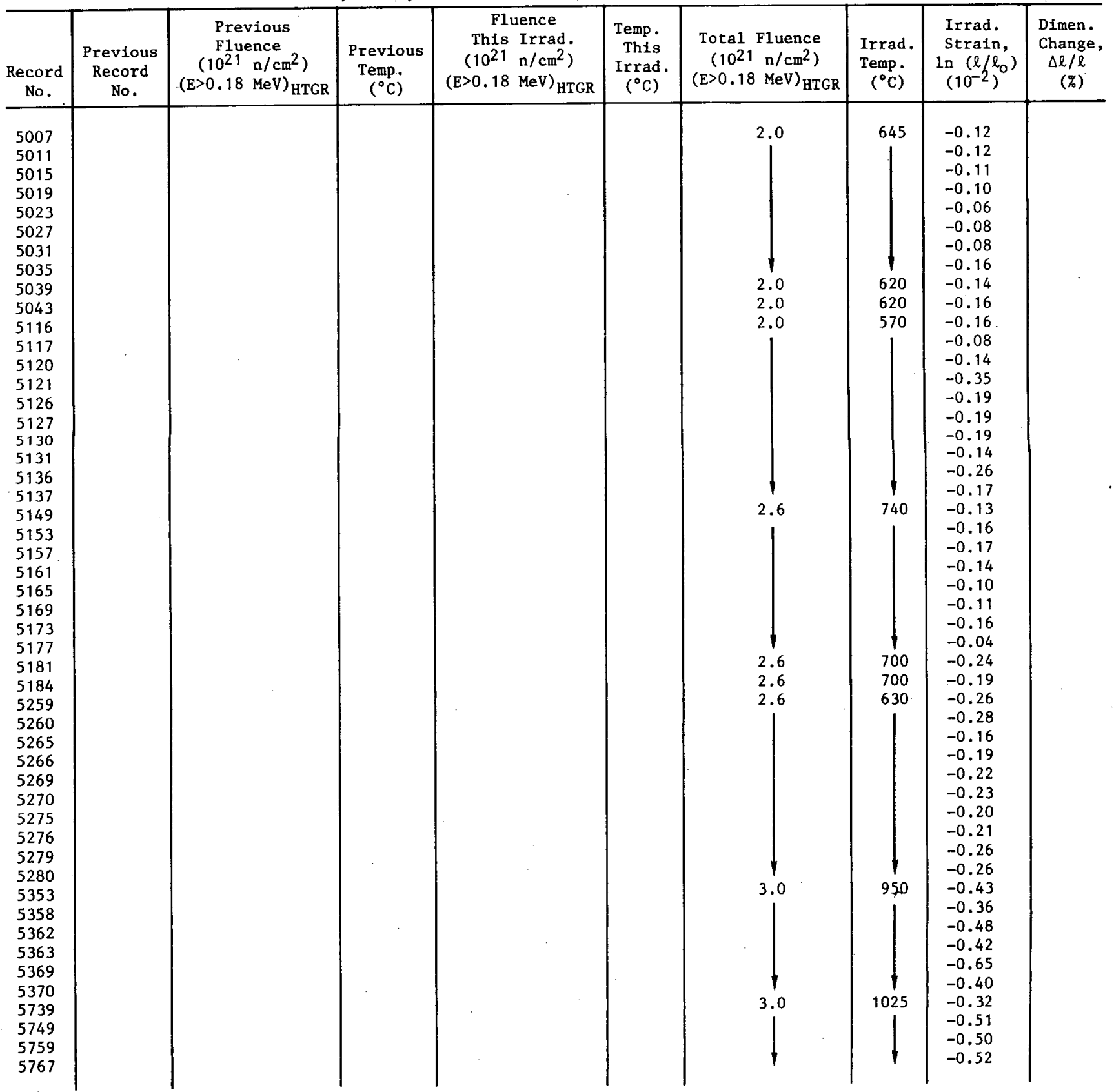


TABLE 5-19 (continued)

\begin{tabular}{|c|c|c|c|c|c|c|c|c|c|}
\hline $\begin{array}{c}\text { Record } \\
\text { No. }\end{array}$ & $\begin{array}{l}\text { Previous } \\
\text { Record } \\
\text { No. }\end{array}$ & $\begin{array}{c}\text { Previous } \\
\text { F1uence } \\
\left(10^{21} \mathrm{n} / \mathrm{cm}^{2}\right) \\
(E>0.18 \mathrm{MeV})_{\text {HTGR }}\end{array}$ & $\begin{array}{l}\text { Previous } \\
\text { Temp. } \\
\left({ }^{\circ} \mathrm{C}\right)\end{array}$ & $\begin{array}{c}\text { Fluence } \\
\text { Th1s Irrad } \\
\left(10^{21} \mathrm{n} / \mathrm{cm}^{2}\right) \\
(\mathrm{E}>0.18 \mathrm{MeV})_{\text {HTGR }}\end{array}$ & $\begin{array}{l}\text { Temp } \\
\text { This } \\
\text { Irrad } \\
\left({ }^{\circ} \mathrm{C}\right)\end{array}$ & $\begin{array}{l}\text { Total Fluence } \\
\left(10^{21} \mathrm{n} / \mathrm{cm}^{2}\right) \\
(E>0.18 \mathrm{MeV})_{\text {HTGR }}\end{array}$ & $\begin{array}{l}\text { Irrad. } \\
\text { Temp. } \\
\left({ }^{\circ} \mathrm{C}\right)\end{array}$ & $\begin{array}{l}\text { Irrad. } \\
\text { Strain, } \\
\ln \left(\ell / \ell_{0}\right) \\
\left(10^{-2}\right)\end{array}$ & $\begin{array}{c}\text { Dimen. } \\
\text { Change, } \\
\Delta \ell / \ell \\
(\%)\end{array}$ \\
\hline $\begin{array}{l}5772 \\
5776 \\
5780 \\
5785 \\
5792 \\
5798 \\
5809 \\
5813 \\
5817 \\
5821 \\
5825 \\
5858 \\
5859 \\
5862 \\
5863 \\
5866 \\
5867 \\
5909 \\
5913 \\
5917 \\
5921 \\
5925 \\
5929 \\
6026 \\
6027 \\
6032 \\
6033 \\
6036 \\
6037 \\
6211 \\
6215 \\
6219 \\
6223 \\
6227 \\
6231 \\
6235 \\
6239 \\
6243 \\
6303 \\
6507 \\
6511 \\
6591 \\
6592 \\
6595 \\
6596 \\
6599 \\
6600 \\
6603\end{array}$ & $\begin{array}{l}5007 \\
5011 \\
5116 \\
5117 \\
5120 \\
5121 \\
5126 \\
5127 \\
5130\end{array}$ & $\begin{array}{r}2.0 \\
2.0 \\
2.0 \\
\end{array}$ & $\begin{array}{l}645 \\
645 \\
570\end{array}$ & $\begin{array}{l}1.7 \\
1.7 \\
1.7\end{array}$ & $\begin{array}{l}680 \\
680 \\
615\end{array}$ & 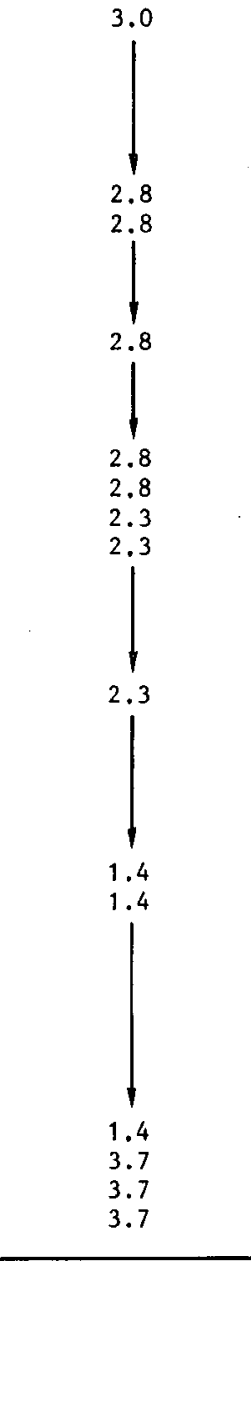 & 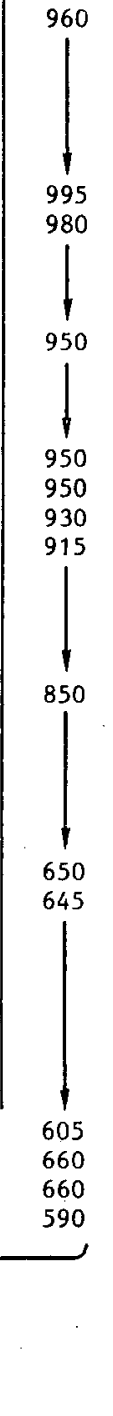 & $\begin{array}{l}-0.36 \\
-0.40 \\
-0.42 \\
-0.41 \\
-0.32 \\
-0.40 \\
-0.30 \\
-0.16 \\
-0.26 \\
-0.35 \\
-0.37 \\
-0.43 \\
-0.12 \\
-0.39 \\
-0.37 \\
-0.37 \\
-0.35 \\
-0.25 \\
-0.12 \\
-0.08 \\
-0.02 \\
-0.24 \\
-0.22 \\
-0.19 \\
-0.12 \\
-0.23 \\
-0.02 \\
-0.14 \\
-0.08 \\
-0\end{array}$ & \\
\hline
\end{tabular}


TABLE 5-19 (continued)

\begin{tabular}{|c|c|c|c|c|c|c|c|c|c|}
\hline $\begin{array}{c}\text { Record } \\
\text { No. }\end{array}$ & $\begin{array}{l}\text { Prevlous } \\
\text { Record } \\
\text { No. }\end{array}$ & $\begin{array}{c}\text { Previous } \\
\text { F1uence } \\
\left(10^{21} \mathrm{n} / \mathrm{cm}^{2}\right) \\
(\mathrm{E}>0.18 \mathrm{MeV})_{\text {HTGR }}\end{array}$ & $\begin{array}{l}\text { Previous } \\
\text { Temp } \\
\left({ }^{\circ} \mathrm{C}\right)\end{array}$ & $\begin{array}{c}\text { Fluence } \\
\text { Th1s Irrad } \\
\left(10^{21} \mathrm{n} / \mathrm{cm}^{2}\right) \\
(E>0.18 \mathrm{MeV})_{\text {HTGR }}\end{array}$ & $\begin{array}{l}\text { Temp. } \\
\text { This } \\
\text { Irrad. } \\
\left({ }^{\circ} \mathrm{C}\right)\end{array}$ & $\begin{array}{c}\text { Total Fluence } \\
\left(10^{21} \mathrm{n} / \mathrm{cm}^{2}\right) \\
(E>0.18 \mathrm{MeV}) \\
\text { HTGR }\end{array}$ & $\begin{array}{l}\text { Irrad. } \\
\text { Temp. } \\
\left({ }^{\circ} \mathrm{C}\right)\end{array}$ & $\begin{array}{l}\text { Irrad. } \\
\text { Strain, } \\
\ln \left(\ell / \ell_{0}\right) \\
\left(10^{-2}\right)\end{array}$ & $\begin{array}{l}\text { Dimen. } \\
\text { Change, } \\
\Delta \ell / \ell \\
(\%)\end{array}$ \\
\hline $\begin{array}{l}6632 \\
6636 \\
6640 \\
6644 \\
6648 \\
6652 \\
6656 \\
6705 \\
6706 \\
6721 \\
6722 \\
6723 \\
6724 \\
6725 \\
6726 \\
6727 \\
6728 \\
6729 \\
6730 \\
6764 \\
6765 \\
6766 \\
6770 \\
6771 \\
6772 \\
7162 \\
7163 \\
7164 \\
7165 \\
7166 \\
7167 \\
7168 \\
7169 \\
7218 \\
7222 \\
7226 \\
7230 \\
7234 \\
7305 \\
7306 \\
7531 \\
7535 \\
7539 \\
7543\end{array}$ & $\begin{array}{l}5809 \\
5813 \\
5817 \\
5821 \\
5825 \\
5858 \\
5859 \\
6211 \\
6215 \\
6219 \\
6223\end{array}$ & $\begin{array}{l}2.6 \\
2.6 \\
\underbrace{3.0}\end{array}$ & $\begin{array}{l}630 \\
630\end{array}$ & $\begin{array}{l}1 \\
2.5 \\
2.5 \\
1.15 \\
1.15\end{array}$ & $\begin{array}{r}680 \\
680\end{array}$ & $\begin{array}{c}4.8 \\
4.8 \\
2.2 \\
1 \\
1 \\
5.7 \\
\end{array}$ & $\begin{array}{c}655 \\
655 \\
680 \\
\mid\end{array}$ & $\begin{array}{l}-0.44 \\
-0.44 \\
-0.44 \\
-0.44 \\
-0.40 \\
-0.44 \\
-0.46 \\
-0.58 \\
-0.58 \\
-0.15 \\
-0.17 \\
-0.15 \\
-0.15 \\
-0.16 \\
-0.18 \\
-0.17 \\
-0.17 \\
-0.15 \\
-0.17 \\
-0.68 \\
-0.62 \\
-0.68 \\
-0.81 \\
-0.93 \\
-0.67 \\
-0.58 \\
-0.48 \\
-0.59 \\
-0.51 \\
-0.60 \\
-0.47 \\
-0.58 \\
-0.43 \\
-0.52 \\
-0.64 \\
-0.72 \\
-0.69 \\
-0.57 \\
-0.72 \\
-0.71 \\
-0.09 \\
-1.38 \\
-0.15 \\
-0.08\end{array}$ & \\
\hline
\end{tabular}


TABLE $5-20$

DIMENSIONAL CHANGE DATA, TREATED H-451 GRAPHITES, AXIAL DIRECTION, AFTER IRRADIATION IN CAPSULE OG-2

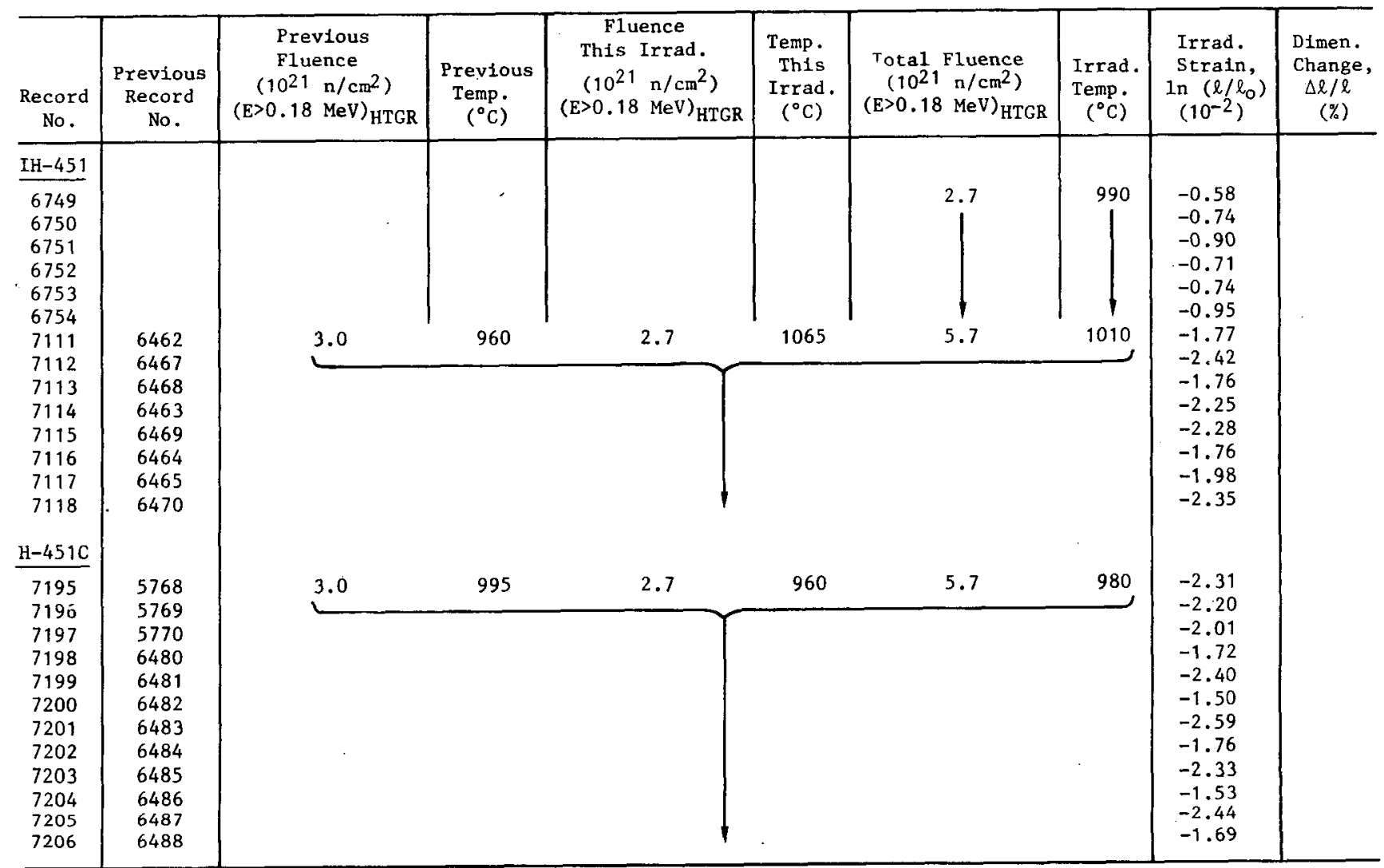


TABLE 5-21

DIMENSIONAL CHANGE DATA FOR B 4 C-GRAPHITE, 2020, AND 565161 GRAPHITES, AXIAL DIRECTION, AFTER IRRADIATION IN CAPSULE OG-2

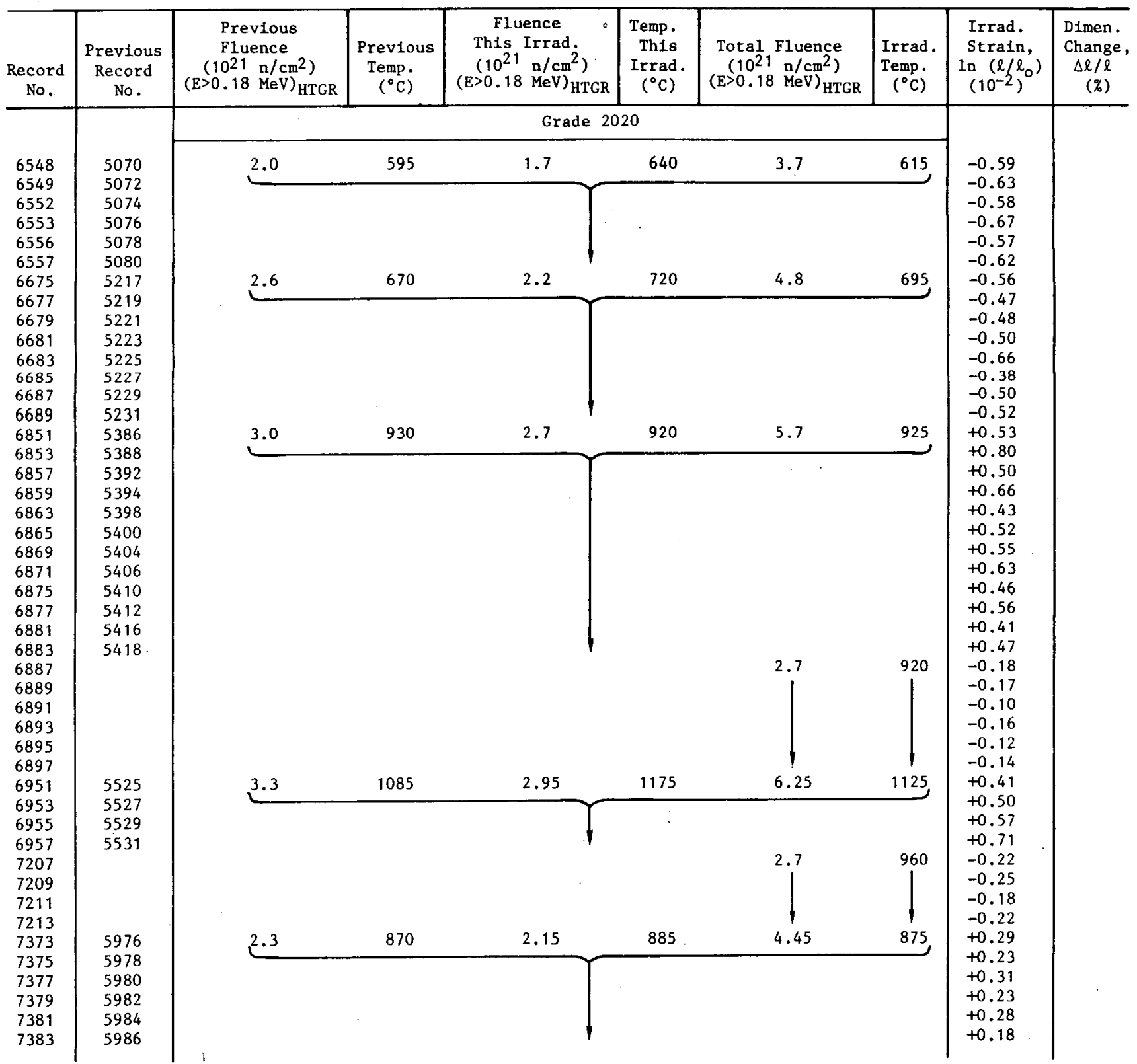


TABLE 5-21 (continued)

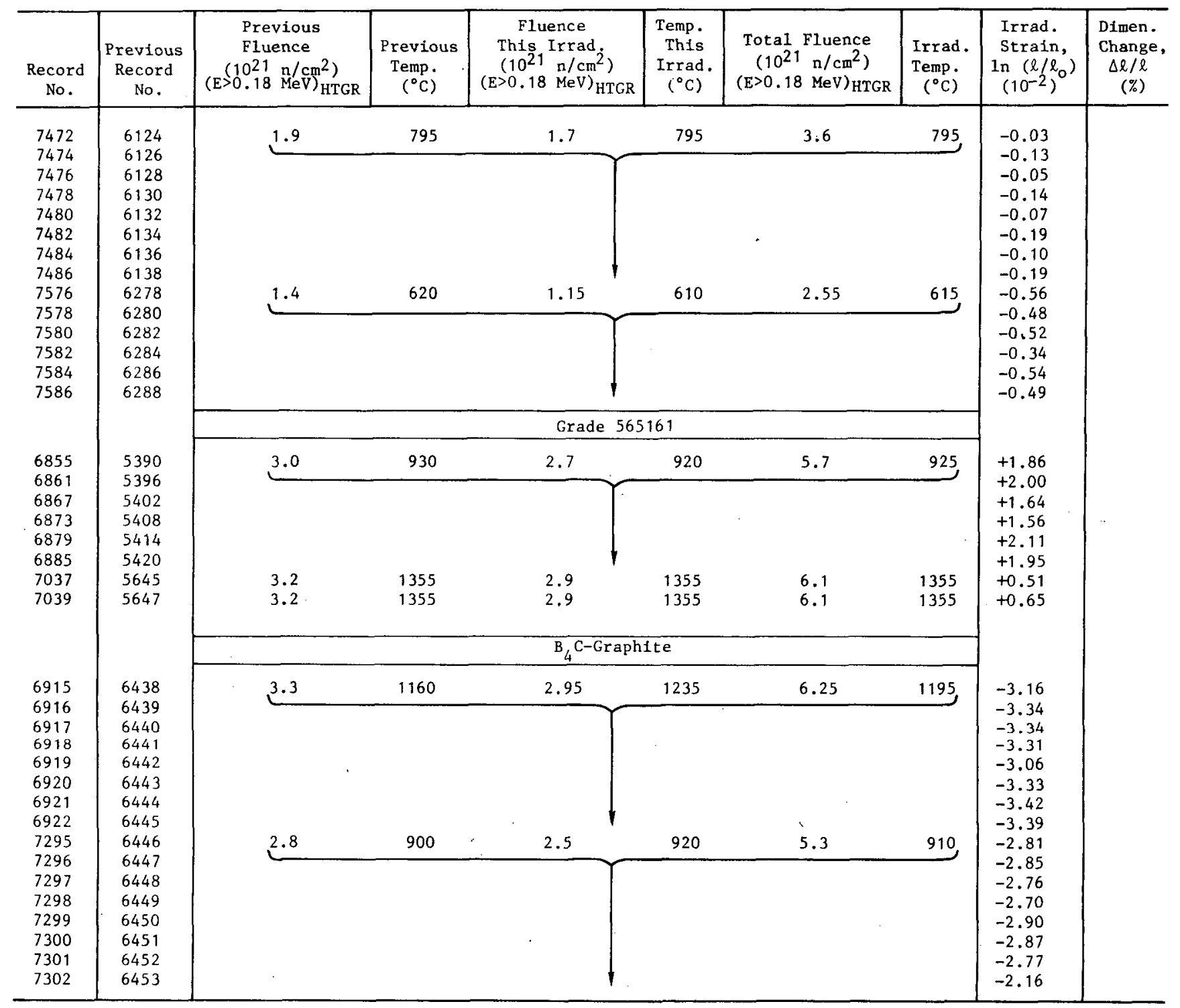


TABLE 5-22

DIMENSIONAL CHANGE DATA FOR 2020 AND 565161 GRAPHITES, RADIAL DIRECTION, AFTER IRRADIATION IN CAPSULE OG-2

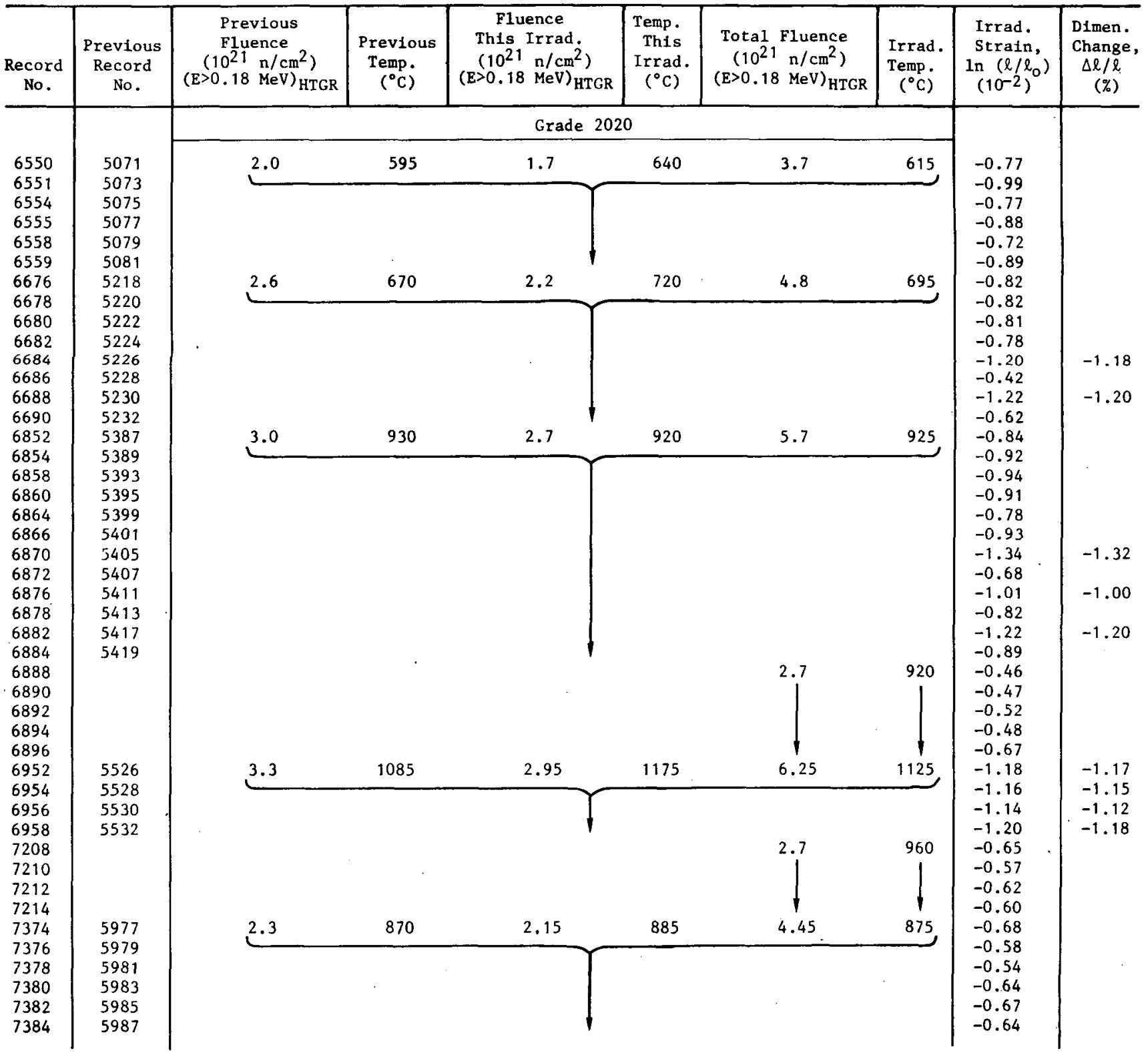


TABLE 5-22 (continued)

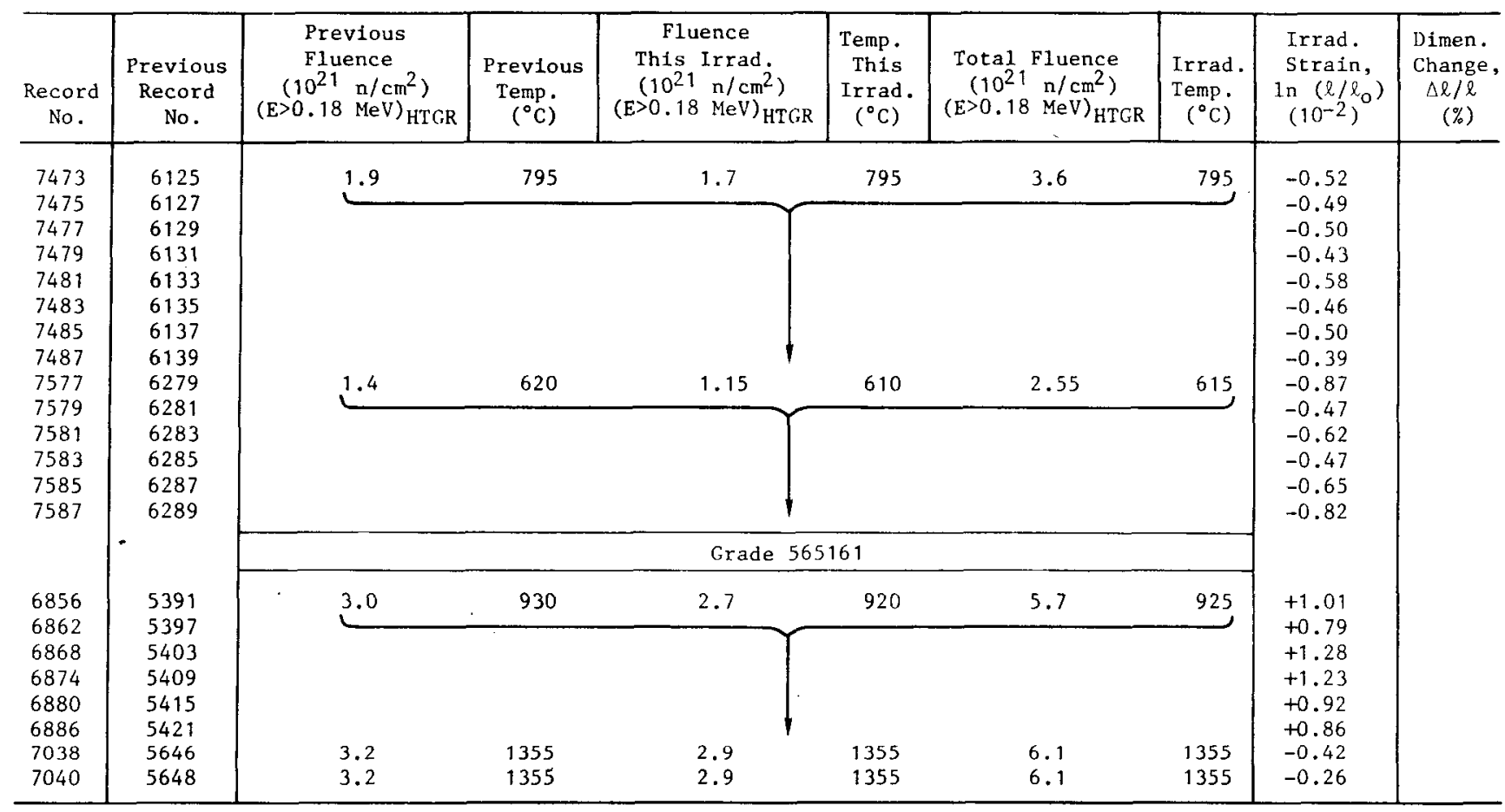


TABLE 5-23

DIMENSIONAL CHANGES IN CARBON INSULATING MATERIALS IRRADIATED IN CAPSULES OG-1 AND OG-2

\begin{tabular}{|c|c|c|c|c|c|c|}
\hline Material & $\begin{array}{l}\text { Specimen } \\
\text { No. }\end{array}$ & Crucible & $\begin{array}{c}\text { Irradiation } \\
\text { Temperature } \\
\left({ }^{\circ} \mathrm{C}\right)\end{array}$ & $\begin{array}{c}\text { Fast } \\
\text { Neutron } \\
\text { Fluence } \\
\left(10^{21} \mathrm{n} / \mathrm{cm}^{2}\right) \\
(E>0.18 \mathrm{MeV})_{\text {HTGR }}\end{array}$ & $\begin{array}{l}\text { Direction of } \\
\text { Measurement }\end{array}$ & $\begin{array}{c}\text { Dimensional } \\
\text { Change } \\
(\%)\end{array}$ \\
\hline $\begin{array}{l}\text { FPA-20 } \\
\text { carbon foam }\end{array}$ & $\begin{array}{l}\text { I } \\
\text { II } \\
\text { I }\end{array}$ & ? & $\begin{array}{c}900 \\
900 \\
900-990\end{array}$ & $\begin{array}{l}2.8 \\
2.8 \\
5.3\end{array}$ & $\begin{array}{l}\text { Axial } \\
\text { Radial } \\
\text { Axial } \\
\text { Radial } \\
\text { Axial } \\
\text { Radial }\end{array}$ & $\begin{array}{l}-6 \\
-8 \\
-9 \\
-11 \\
-7 \\
-11\end{array}$ \\
\hline Grafoil & (10 specimens) & $\begin{array}{l}5 \\
5\end{array}$ & $\begin{array}{l}1350 \\
1350\end{array}$ & $\begin{array}{l}3.2 \\
6.1\end{array}$ & $\begin{array}{l}\text { In-plane } \\
\text { In-plane }\end{array}$ & $\begin{array}{r}0 \text { to }-5 \\
+3 \text { to }-6\end{array}$ \\
\hline Palarite C & $\begin{array}{l}\text { I } \\
\text { II }\end{array}$ & 7 & $\begin{array}{c}900 \\
900 \\
900 \\
900-990 \\
900-990\end{array}$ & $\begin{array}{l}2.8 \\
2.8 \\
2.8 \\
\\
5.3 \\
5.3\end{array}$ & $\begin{array}{l}\text { Parallel to layers } \\
\text { Parallel to layers } \\
\text { Perpendicular to } \\
\text { layers } \\
\text { Parallel to layers } \\
\text { Perpendicular to } \\
\text { layers }\end{array}$ & $\begin{array}{l}-11 \\
-11 \\
+15 \\
\\
-14 \\
+20\end{array}$ \\
\hline
\end{tabular}




\section{EXPERIMENTAL RESULTS - THERMAL PROPERTIES}

\subsection{THERMAL EXPANSIVITY}

Thermal expansivity measurements were made on 98 specimens of irradiated graphite measuring either 0.2 in. in diameter by 0.45 in. long or 0.25 in. in diameter by $0.9 \mathrm{in.}$ long. Measurements were made in a silica dilatometer between room temperature and $100^{\circ} \mathrm{C}$ less than the irradiation temperature (maximum $1000^{\circ} \mathrm{C}$ ). The graphites tested were near-isotropic grades H-451, H-429 (subsize prototype of H-451), and $\mathrm{TS}-1240$ and needle coke grade $\mathrm{H}-327$. The $\mathrm{H}-451, \mathrm{H}-429$, and $\mathrm{H}-327$ graphite specimens had been irradiated in previous capsules, and lower-fluence thermal expansivity data are given in Ref. 4 .

The thermal expansivities between room temperature and $500^{\circ} \mathrm{C}$ are listed in Tables $6-1$ through $6-3$, together with mean values for unirradiated material. The fractional changes in thermal expansivity are plotted as functions of fluence for the near-isotropic graphites in Fig. 6-1 and for H-327 (needle coke) graphite in Fig. 6-2. Figures $6-1$ and 6-2 also include data obtained during postirradiation tests on specimens from capsule OG-1 (Ref. 4). The near-isotropic graphites (Fig. 6-1) show a small increase in thermal expansivity (generally less than 10\%) in both the axial and radial directions when irradiated at $590^{\circ}$ to $680^{\circ} \mathrm{C}$ and no significant changes when irradiated at $765^{\circ}$ to $925^{\circ} \mathrm{C}$. At higher irradiation temperatures, there is a pronounced decrease in thermal expansivity, with the values falling $30 \%$ after irradiation to about $7 \times 10^{21} \mathrm{n} / \mathrm{cm}^{2}$ above $1100^{\circ} \mathrm{C}$. These irradiation-induced changes are similar in nature to, but smaller in magnitude than, those reported for Gilsocarbon-based isotropic nuclear graphites. H-327 graphite showed a small decrease in thermal expansivity on irradiation, with some indication of recovery at high fluences for the highest irradiation temperatures (Fig. 6-2). 
TABLE 6-1

THERMAL EXPANSIVITY OF H-429 AND H-451 GRAPHITE SPECIMENS IRRADIATED IN CAPSULE OG-2

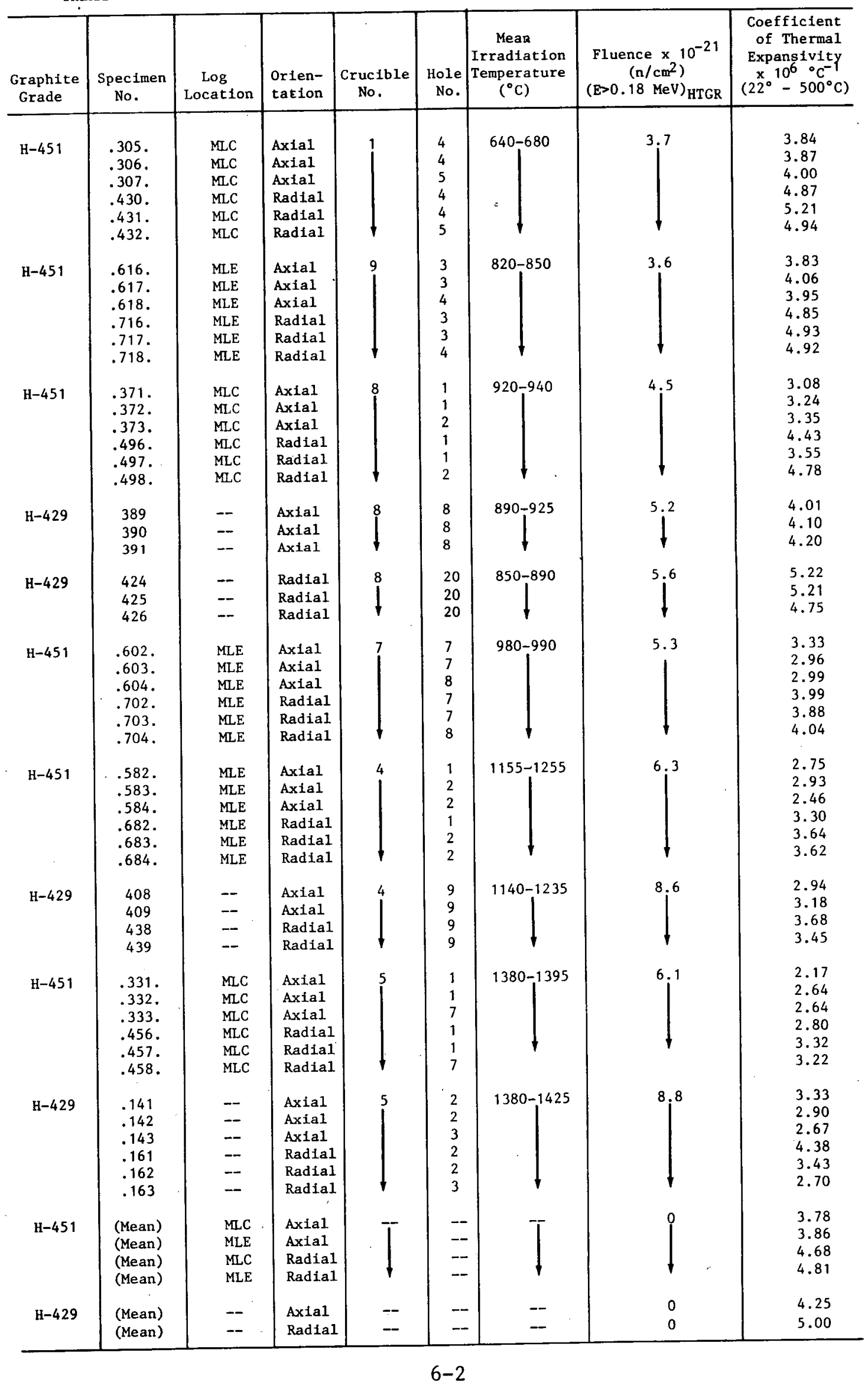


TABLE 6-2

THERMAL EXPANSIVITY OF TS-1240 GRAPHITE SPECIMENS IRRADIATED IN CAPSULE OG-2

\begin{tabular}{|c|c|c|c|c|c|c|c|}
\hline $\begin{array}{l}\text { Speclmen } \\
\text { No. }\end{array}$ & $\begin{array}{c}\text { Log } \\
\text { Lopation }\end{array}$ & $\begin{array}{l}\text { Orien- } \\
\text { tation }\end{array}$ & $\begin{array}{c}\text { Crucible } \\
\text { No. }\end{array}$ & $\begin{array}{c}\text { Hole } \\
\text { No. }\end{array}$ & $\begin{array}{l}\text { Mean } \\
\text { Irradiation } \\
\text { Temperature } \\
\quad\left({ }^{\circ} \mathrm{C}\right)\end{array}$ & $\begin{array}{c}\text { Fluence } \times 10^{-21} \\
\left(\mathrm{n} / \mathrm{cm}^{2}\right) \\
(\mathrm{E}>0.18 \mathrm{MeV})_{\text {HTGR }}\end{array}$ & $\begin{array}{l}\text { Coefficient } \\
\text { of Thermal } \\
\text { Expansivity } \\
\times 10^{\circ}{ }^{\circ} \mathrm{C} 1 \\
\left(22^{\circ}-500^{\circ} \mathrm{C}\right)\end{array}$ \\
\hline $\begin{array}{l}\text { T40105 } \\
\text { T40107 } \\
\text { T40109 } \\
\text { T40149 } \\
\text { T40151 } \\
\text { T40153 }\end{array}$ & $\begin{array}{l}\text { MLC } \\
\text { MLC } \\
\text { MLC } \\
\text { MLC } \\
\text { MLC } \\
\text { MLC }\end{array}$ & $\begin{array}{l}\text { Axial } \\
\text { Axial } \\
\text { Axial } \\
\text { Radial } \\
\text { Radial } \\
\text { Radial }\end{array}$ & 1 & $\begin{array}{l}10 \\
10 \\
11 \\
32 \\
32 \\
33\end{array}$ & $\downarrow_{590}^{620}$ & $i^{1.2}$ & $\begin{array}{l}4.18 \\
4.26 \\
5.25 \\
4.89 \\
4.86 \\
4.66\end{array}$ \\
\hline $\begin{array}{l}\text { T40069 } \\
\text { T40071 } \\
\text { T40073 } \\
\text { T40129 } \\
\text { T40131 } \\
\text { T40133 }\end{array}$ & $\begin{array}{l}\text { MLC } \\
\text { MLC } \\
\text { MLC } \\
\text { MLC } \\
\text { MLC } \\
\text { MLC }\end{array}$ & $\begin{array}{l}\text { Axial } \\
\text { Axial } \\
\text { Axial } \\
\text { Radial } \\
\text { Radial } \\
\text { Radial }\end{array}$ & 9 & $\begin{array}{l}28 \\
28 \\
29 \\
12 \\
12 \\
13\end{array}$ & $\left.\right|_{805} ^{765}$ & $\begin{array}{c}1.7 \\
1\end{array}$ & $\begin{array}{l}4.14 \\
4.53 \\
4.30 \\
4.66 \\
4.47 \\
4.86\end{array}$ \\
\hline $\begin{array}{l}\mathrm{T} 40053 \\
\mathrm{~T} 40055 \\
\mathrm{~T} 40057 \\
\mathrm{~T} 40121 \\
\mathrm{~T} 40123 \\
\mathrm{~T} 40125\end{array}$ & $\begin{array}{l}\text { MLC } \\
\text { MLC } \\
\text { MLC } \\
\text { MLC } \\
\text { MLC } \\
\text { MLC }\end{array}$ & $\begin{array}{l}\text { Axial } \\
\text { Axial } \\
\text { Axial } \\
\text { Radial } \\
\text { Radial } \\
\text { Radial }\end{array}$ & $\begin{array}{l}7 \\
1\end{array}$ & $\begin{array}{l}25 \\
25 \\
26 \\
29 \\
29 \\
30\end{array}$ & 920 & 2.5 & $\begin{array}{l}4.69 \\
4.06 \\
3.90 \\
4.67 \\
4.73 \\
4.55\end{array}$ \\
\hline $\begin{array}{l}\mathrm{T} 40017 \\
\mathrm{~T} 40019 \\
\mathrm{~T} 40021 \\
\mathrm{~T} 40157 \\
\mathrm{~T} 40159 \\
\mathrm{~T} 40161\end{array}$ & $\begin{array}{l}\text { MLC } \\
\text { MLC } \\
\text { MLC } \\
\text { MLC } \\
\text { MLC } \\
\text { MLC }\end{array}$ & $\begin{array}{l}\text { Axial } \\
\text { Axtal } \\
\text { Axial } \\
\text { Radial } \\
\text { Radial } \\
\text { Radial }\end{array}$ & 4 & $\begin{array}{l}28 \\
28 \\
29 \\
10 \\
10 \\
11\end{array}$ & $\left.\right|_{1200} ^{1105}$ & 2.9 & $\begin{array}{l}3.98 \\
4.40 \\
4.49 \\
4.15 \\
4.67 \\
4.12\end{array}$ \\
\hline $\begin{array}{l}\text { (Mean) } \\
\text { (Mean) }\end{array}$ & $\begin{array}{l}\text { MLC } \\
\text { MLC }\end{array}$ & $\begin{array}{l}\text { Axial } \\
\text { Radial }\end{array}$ & - & -- & -- & $\begin{array}{l}0 \\
0\end{array}$ & $\begin{array}{l}4.26 \\
4.84\end{array}$ \\
\hline
\end{tabular}


TABLE 6-3

THERMAL EXPANSIVITY OF H-327 GRAPHITE SPECIMENS IRRADIATED IN CAPSULE OG-2

\begin{tabular}{|c|c|c|c|c|c|c|c|}
\hline $\begin{array}{l}\text { Specimen } \\
\text { No. }\end{array}$ & $\begin{array}{c}\text { Log } \\
\text { Location }\end{array}$ & $\begin{array}{l}\text { Orien- } \\
\text { tation }\end{array}$ & $\begin{array}{c}\text { Crucible } \\
\text { No. }\end{array}$ & $\begin{array}{c}\text { Hole } \\
\text { No. }\end{array}$ & $\begin{array}{c}\text { Mean } \\
\text { Irradiation } \\
\text { Temperature } \\
\left({ }^{\circ} \mathrm{C}\right)\end{array}$ & $\begin{array}{c}\text { Fluence } \times 10^{-21} \\
\left(\mathrm{n} / \mathrm{cm}^{2}\right) \\
\text { (E } 0.18 \mathrm{MeV})_{\text {HTGR }}\end{array}$ & $\begin{array}{l}\text { Coefficient } \\
\text { of Thermal } \\
\text { Expansivity } \\
x 10^{6}{ }^{\circ} \mathrm{C}^{-1} \\
\left(22^{\circ}-500^{\circ} \mathrm{C}\right)\end{array}$ \\
\hline $\begin{array}{l}.203 . \\
.204 . \\
.205 . \\
.253 . \\
.254 . \\
.255\end{array}$ & $\begin{array}{l}\text { MLE } \\
\text { MLE } \\
\text { MLE } \\
\text { MLE } \\
\text { EMLE } \\
\text { MLE }\end{array}$ & $\begin{array}{l}\text { Axial } \\
\text { Axial } \\
\text { Axial } \\
\text { Radial } \\
\text { Radial } \\
\text { Radial }\end{array}$ & 1 & $\begin{array}{l}21 \\
21 \\
22 \\
21 \\
21 \\
22\end{array}$ & $\begin{array}{c}560-615 \\
\mid\end{array}$ & 1 & $\begin{array}{l}1.58 \\
1.34 \\
1.58 \\
3.94 \\
3.49 \\
3.63\end{array}$ \\
\hline $\begin{array}{l}140 \\
141 \\
138 \\
139\end{array}$ & $\begin{array}{l}\text { MLC } \\
\text { MLE } \\
\text { MLC } \\
\text { MLE }\end{array}$ & $\begin{array}{l}\text { Axial } \\
\text { Axial } \\
\text { Radial } \\
\text { Radial }\end{array}$ & 8 & $\begin{array}{l}6 \\
6 \\
6 \\
6\end{array}$ & $\left.\right|^{890-1225}$ & $i^{8.5}$ & $\begin{array}{l}2.28 \\
1.60 \\
3.36 \\
3.87\end{array}$ \\
\hline $\begin{array}{l}.225 . \\
.28 . \\
.275 \\
.127 . \\
.128 .\end{array}$ & $\begin{array}{l}\text { MLE } \\
\text { MLC } \\
\text { MLE } \\
\text { MLC } \\
\text { MLC }\end{array}$ & $\begin{array}{l}\text { Axial } \\
\text { Axial } \\
\text { Radial } \\
\text { Radial } \\
\text { Radial }\end{array}$ & 7 & $\begin{array}{r}5 \\
21 \\
5 \\
21 \\
21\end{array}$ & $\begin{array}{l}980-990 \\
920-945 \\
980-990 \\
920-945 \\
920-945\end{array}$ & $i^{5.3}$ & $\begin{array}{l}1.70 \\
1.55 \\
2.98 \\
3.53 \\
3.50\end{array}$ \\
\hline $\begin{array}{c}\mathrm{T}-263 \\
\mathrm{~T}-264 \\
\mathrm{~T}-265 \\
216 \\
217 \\
214 \\
215\end{array}$ & $\begin{array}{l}\text { MLC } \\
\text { MLC } \\
\text { MLC } \\
\text { MLC } \\
\text { MLE } \\
\text { MLC } \\
\text { MLE }\end{array}$ & $\begin{array}{l}\text { Axial } \\
\text { Axial } \\
\text { Axial } \\
\text { Axial } \\
\text { Axial } \\
\text { Radial } \\
\text { Radial }\end{array}$ & 4 & $\begin{array}{r}21 \\
21 \\
22 \\
3 \\
3 \\
3 \\
3\end{array}$ & $\mid$ & $i_{i}^{6.3}$ & $\begin{array}{l}1.21 \\
1.27 \\
1.20 \\
2.29 \\
2.06 \\
3.62 \\
4.33\end{array}$ \\
\hline $\begin{array}{l}\text { (Mean) } \\
\text { (Mean) }\end{array}$ & -- & $\begin{array}{l}\text { Axial } \\
\text { Radial }\end{array}$ & - & - & -- & $\begin{array}{l}0 \\
0\end{array}$ & $\begin{array}{l}1.96 \\
3.70\end{array}$ \\
\hline
\end{tabular}




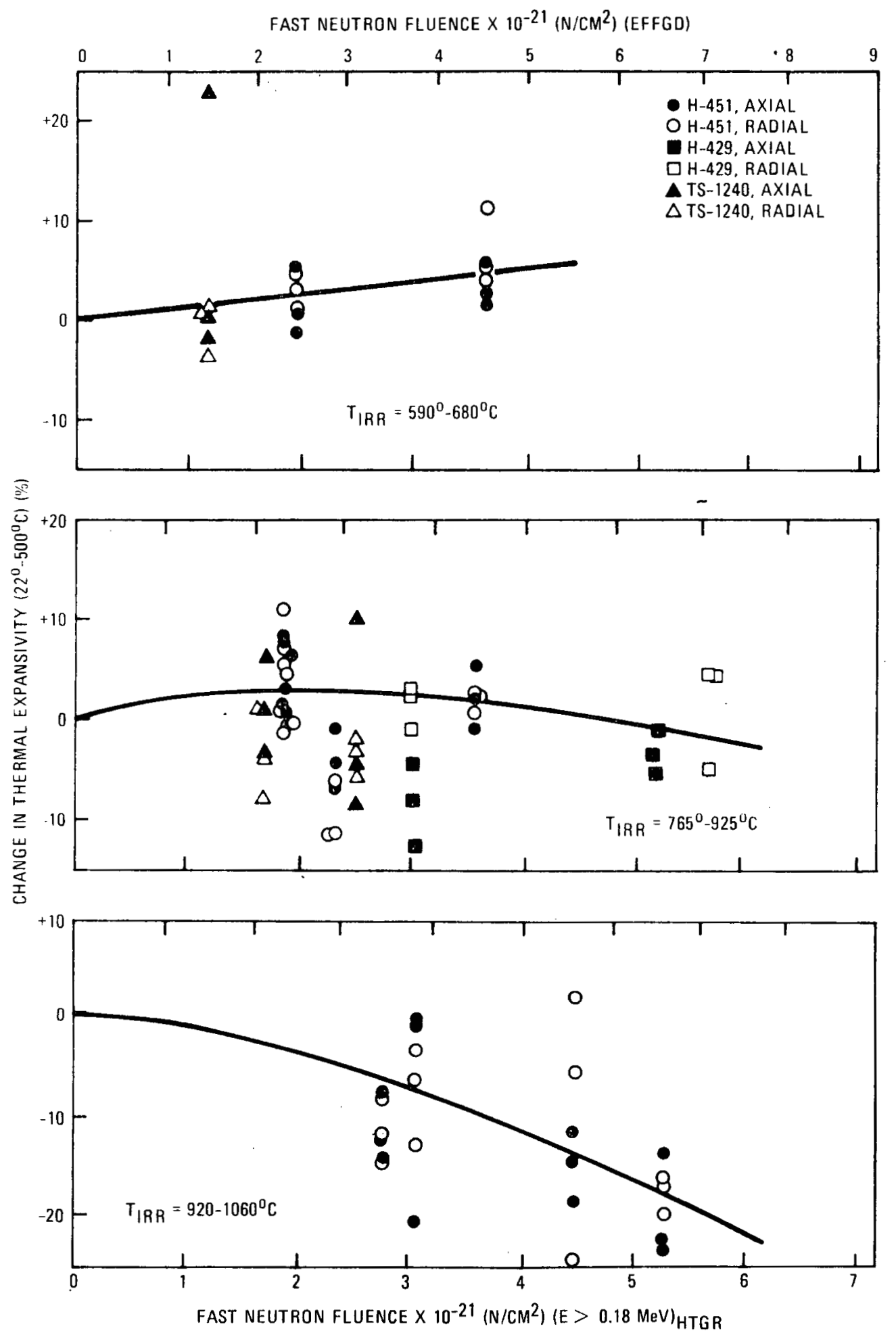

Fig. 6-1(a). Percent change in thermal expansivity $\left(22^{\circ}-500^{\circ} \mathrm{C}\right)$ for $\mathrm{H}-451$ and TS-1240 near-isotropic graphites as a function of fast neutron fluence at irradiation temperatures of $590^{\circ}$ to $1060^{\circ} \mathrm{C}$ 


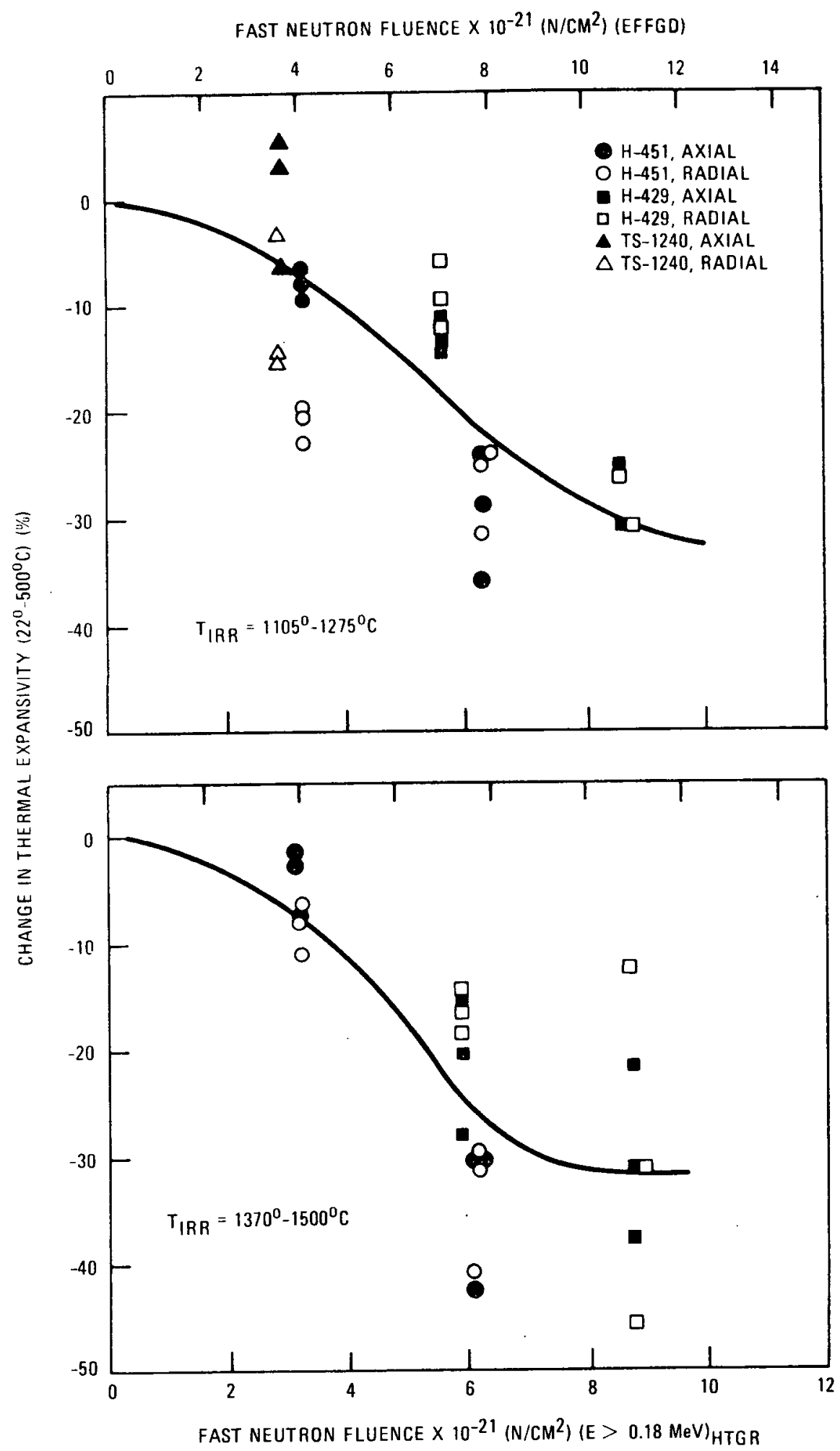

Fig. 6-1(b). Percent change in thermal expansivity $\left(22^{\circ}-500^{\circ} \mathrm{C}\right)$ for $\mathrm{H}-451$ and TS-1240 near-isotropic graphites as a function of fast neutron fluence at irradiation temperatures of $1105^{\circ}$ to $1500^{\circ} \mathrm{C}$ 
FAST NEUTRON FLUENCE $\times 10^{-21}\left(\mathrm{~N} / \mathrm{CM}^{2}\right)$ (EFFGD)
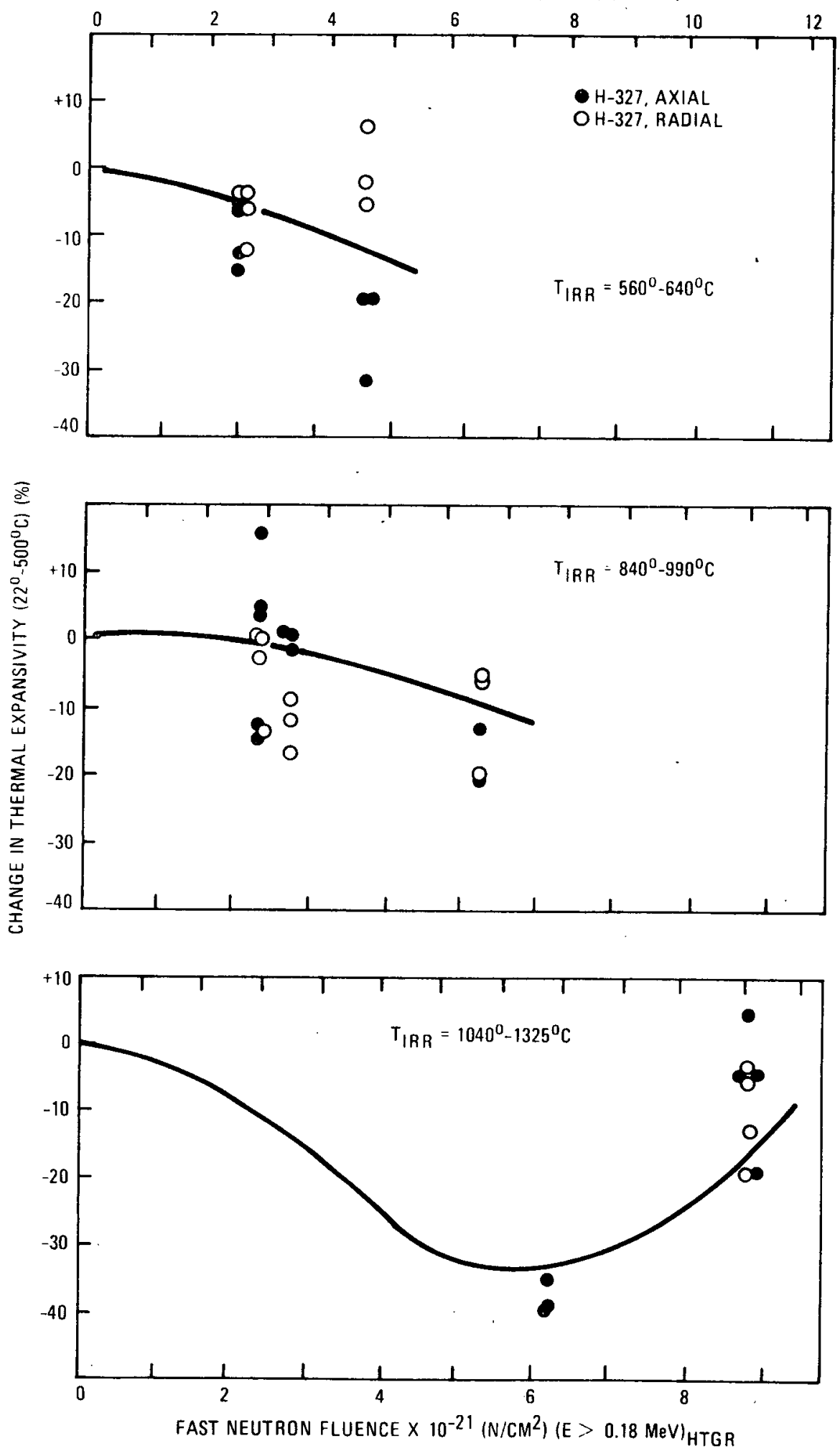

Fig. 6-2. Percent change in thermal expansivity $\left(22^{\circ}-500^{\circ} \mathrm{C}\right)$ for $\mathrm{H}-327$ needle-coke graphite as a function of fast neutron fluence 


\subsection{THERMAL CONDUCTIVITY}

Thermal diffusivity measurements were made on 87 disks $(0.45$ in. in diameter by $0.05 \mathrm{in.} \mathrm{thick)} \mathrm{of} \mathrm{graphite} \mathrm{irradiated} \mathrm{in} \mathrm{three} \mathrm{crucibles} \mathrm{in}$ the OG-2 capsule. The graphite grades were near-isotropic H-451 and TS-1240 and needle coke $\mathrm{H}-327$; both axial and radial specimens were tested. The H-451 and H-327 specimens had been irradiated previously in the OG-1 capsule, and lower-fluence data are reported in Ref. 4. Thermal diffusivity measurements were made by the heat-pulse method (ASTM Method of Test $(-741)$, and thermal conductivity values were calculated by multiplying the thermal diffusivity by the density of the irradiated specimen and values for the heat capacity taken from the literature. Readings were taken at $100^{\circ} \mathrm{C}$ intervals between room temperature and $100^{\circ} \mathrm{C}$ below the irradiation temperature (maximum $800^{\circ} \mathrm{C}$ ).

The measured values are given in Tables 6-4 through 6-6, together with extrapolated values at the irradiation temperature. The thermal conductivity at the irradiation temperature is plotted as a function of fast fluence in Fig. 6-3 for $\mathrm{H}-327$ (needle coke) graphite. Figures 6-3 and 6-4 also include data from previous capsules GEH-13-422 and OG-1 (Ref. 4). Irradiation reduces the thermal conductivity to a saturation level which increases with increasing irradiation temperature. For fluences above about $7 \times 10^{21} \mathrm{n} / \mathrm{cm}^{2}$ at $1225^{\circ}$ to $1350^{\circ} \mathrm{C}, \mathrm{H}-327$ graphite undergoes a second decline in thermal conductivity as the density of the material is reduced. The effect of grain orientation on the conductivity of the oriented needle coke H-327 is evident in Fig. 6-4. There is no significant difference between the thermal conductivities of irradiated $\mathrm{H}-451$ graphite and irradiated TS-1240 graphite.

For design purposes, it is convenient to express the thermal conductivity of irradiated graphite, $\mathrm{K}_{j}$, as an approach to saturation (Ref. 7):

$$
\mathrm{K}_{i}=\mathrm{K}_{\text {sat }}(\mathrm{T})+\left[\mathrm{K}_{\mathrm{o}}-\mathrm{K}_{\text {sat }}(\mathrm{T})\right] \exp \left[-\frac{\phi t}{\tau(T)}\right] \text {, }
$$


TABLE 6-4

THERMAL CONDUCTIVITY OF H-451 GRAPHITE SPECIMENS IRRADIATED IN CAPSULE OG-2 (Al1 Specimens at Midlength-Center Location)

\begin{tabular}{|c|c|c|c|c|c|c|c|c|c|c|}
\hline \multirow[b]{2}{*}{$\begin{array}{c}\text { Specimen } \\
\text { No. }\end{array}$} & \multirow[b]{2}{*}{$\begin{array}{l}\text { Orien- } \\
\text { tation }\end{array}$} & \multirow[b]{2}{*}{$\begin{array}{c}\text { Crucible } \\
\text { No. }\end{array}$} & \multirow{2}{*}{$\begin{array}{l}\text { Mean } \\
\text { Irradiation } \\
\text { Temperature } \\
\quad\left({ }^{\circ} \mathrm{C}\right)\end{array}$} & \multirow[b]{2}{*}{$\begin{array}{c}\text { F1uence } \times 10^{-21} \\
\left(\mathrm{n} / \mathrm{cm}^{2}\right) \\
(\mathrm{E}>0.18 \mathrm{MeV})_{\text {HTGR }}\end{array}$} & \multicolumn{6}{|c|}{ Therma1 Conductivity $\left(\mathrm{cal} / \mathrm{cm}-\mathrm{sec}-{ }^{\circ} \mathrm{C}\right)$} \\
\hline & & & & & $22^{\circ} \mathrm{C}$ & $200^{\circ} \mathrm{C}$ & $400^{\circ} \mathrm{C}$ & $600^{\circ} \mathrm{C}$ & $800^{\circ} \mathrm{C}$ & $\begin{array}{l}\text { Irradiation } \\
\text { Temperature } \\
\text { (Extrapolation) }\end{array}$ \\
\hline 201 & Axial & 1 & $600-635$ & 3.7 & 0.069 & 0.077 & 0.064 & -- & - & 0.064 \\
\hline 202 & Axial & 1 & & & 0.072 & 0.080 & 0.079 & -- & - & 0.077 \\
\hline 203 & Axial & & & & 0.073 & 0.095 & 0.095 & -- & -- & 0.088 \\
\hline 204 & Axial & & & & 0.074 & 0.089 & 0.086 & -- & -- & 0.082 \\
\hline 221 & Radial & & & & 0.067 & -- & -- & -- & -- & -- \\
\hline 222 & Radial & & & & 0.055 & -- & -- & -- & -- & -- \\
\hline 223 & Radial & & & & 0.068 & -- & -- & -- & - & -- \\
\hline 224 & Radial & & & & 0.068 & -- & - & $\ldots$ & -- & -- \\
\hline 225 & Radial & & & & 0.060 & 0.101 & 0.083 & -- & -- & 0.080 \\
\hline 226 & Radial & & & & 0.067 & 0.074 & 0.076 & -- & -- & 0.067 \\
\hline 227 & Radial & & & & 0.065 & 0.081 & 0.082 & -- & - & 0.073 \\
\hline 228 & Radial & 1 & 1 & 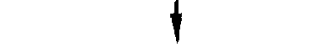 & 0.081 & 0.071 & 0.067 & - & -- & 0.062 \\
\hline 205 & Axial & 7 & $920-945$ & 5.3 & 0.069 & 0.085 & 0.083 & 0.097 & 0.095 & 0.085 \\
\hline 206 & Axial & 1 & & & 0.104 & 0.132 & 0.127 & 0.127 & 0.113 & 0.100 \\
\hline 207 & Axial & & & & 0.109 & 0.132 & 0.129 & 0.118 & 0.113 & 0.100 \\
\hline 208 & Axial & & & & 0.113 & 0.137 & 0.122 & 0.114 & 0.103 & 0.095 \\
\hline 231 & Radial & & & & 0.076 & - & -- & - & -- & -- \\
\hline 232 & Radial & & & & 0.077 & -- & -- & -- & -- & -- \\
\hline 233 & Radial & & & 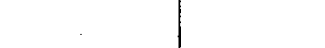 & 0.069 & -- & -- & -- & -- & -- \\
\hline 234 & Radial & & & & 0.100 & -- & $\ldots$ & -- & - & -- \\
\hline 235 & Radial & & & & 0.087 & 0.090 & 0.085 & 0.087 & 0.082 & 0.076 \\
\hline 236 & Radial & & & & 0.091 & 0.100 & 0.086 & $0.08 \mathrm{C}$ & 0.075 & 0.071 \\
\hline 237 & Radial & & & & 0.080 & 0.098 & 0.085 & 0.077 & 0.085 & 0.071 \\
\hline 238 & Radial & 1 & 1 & 1 & 0.073 & 0.081 & 0.078 & 0.071 & 0.068 & 0.064 \\
\hline
\end{tabular}


TABLE 6-4 (Continued)

\begin{tabular}{|c|c|c|c|c|c|c|c|c|c|c|}
\hline \multirow[b]{2}{*}{$\begin{array}{c}\text { Specimen } \\
\text { No. }\end{array}$} & \multirow[b]{2}{*}{$\begin{array}{l}\text { Orien- } \\
\text { tation }\end{array}$} & \multirow[b]{2}{*}{$\begin{array}{c}\text { Crucible } \\
\text { No. }\end{array}$} & \multirow[b]{2}{*}{$\begin{array}{l}\quad \text { Mean } \\
\text { Irradiation } \\
\text { Temperature } \\
\quad\left({ }^{\circ} \mathrm{C}\right)\end{array}$} & \multirow[b]{2}{*}{$\begin{array}{c}\text { Fluence } \times 10^{-21} \\
\left(\mathrm{n} / \mathrm{cm}^{2}\right) \\
(E>0.18 \mathrm{MeV})_{\text {HTGR }}\end{array}$} & \multicolumn{6}{|c|}{ Thermal Conductivity $\left(\mathrm{cal} / \mathrm{cm}-\sec -{ }^{\circ} \mathrm{C}\right)$} \\
\hline & & & & & $22^{\circ} \mathrm{C}$ & $200^{\circ} \mathrm{C}$ & $400^{\circ} \mathrm{C}$ & $600^{\circ} \mathrm{C}$ & $800^{\circ} \mathrm{C}$ & $\begin{array}{l}\text { Irradiation } \\
\text { Temperature } \\
\text { (Extrapolated) }\end{array}$ \\
\hline 210 & Axial & 5 & 1350 & 6.1 & 0.198 & 0.213 & 0.183 & 0.161 & 0.137 & 0.095 \\
\hline 211 & Axial & 1 & 1 & 1 & 0.204 & 0.183 & 0.176 & 0.169 & 0.149 & 0.098 \\
\hline 212 & Axial & & . & & 0.188 & 0.208 & 0.183 & 0.158 & 0.154 & 0.098 \\
\hline 213 & Axial & & & & 0.183 & 0.196 & 0.184 & 0.165 & 0.152 & 0.098 \\
\hline 242 & Radial & & & & 0.140 & - & -- & -- & -- & -- \\
\hline 243 & Radial & & & & 0.136 & -- & -- & -- & -- & -- \\
\hline 244 & Radial & & & & 0.150 & - & -- & -- & -- & -- \\
\hline 245 & Radial & & & & 0.135 & -- & -- & -- & -- & -- \\
\hline 246 & Radial & & & & 0.187 & 0.177 & 0.161 & 0.142 & 0.133 & 0.090 \\
\hline 247 & Radial & & & & 0.170 & 0.169 & 0.145 & 0.130 & 0.128 & 0.084 \\
\hline 248 & Radial & & & & 0.173 & 0.185 & 0.170 & 0.145 & 0.143 & 0.092 \\
\hline 249 & Radial & 1 & 1 & & 0.166 & 0.183 & 0.147 & 0.136 & 0.121 & 0.082 \\
\hline (Mean of 12) & Axial & -- & -- & 0 & 0.347 & $0: 302$ & C. 247 & 0.206 & 0.174 & $-\infty$ \\
\hline (Mean of 12) & Radial & - & -- & 0 & 0.294 & 0.262 & 0.222 & 0.185 & 0.158 & -- \\
\hline
\end{tabular}


TABLE 6-5

THERMAL CONDUCTIVITY OF TS-1240 GRAPHITE SPECIMENS IRRADIATED IN CAPSULE OG-2

(All Specimens at Midlength-Center Location)

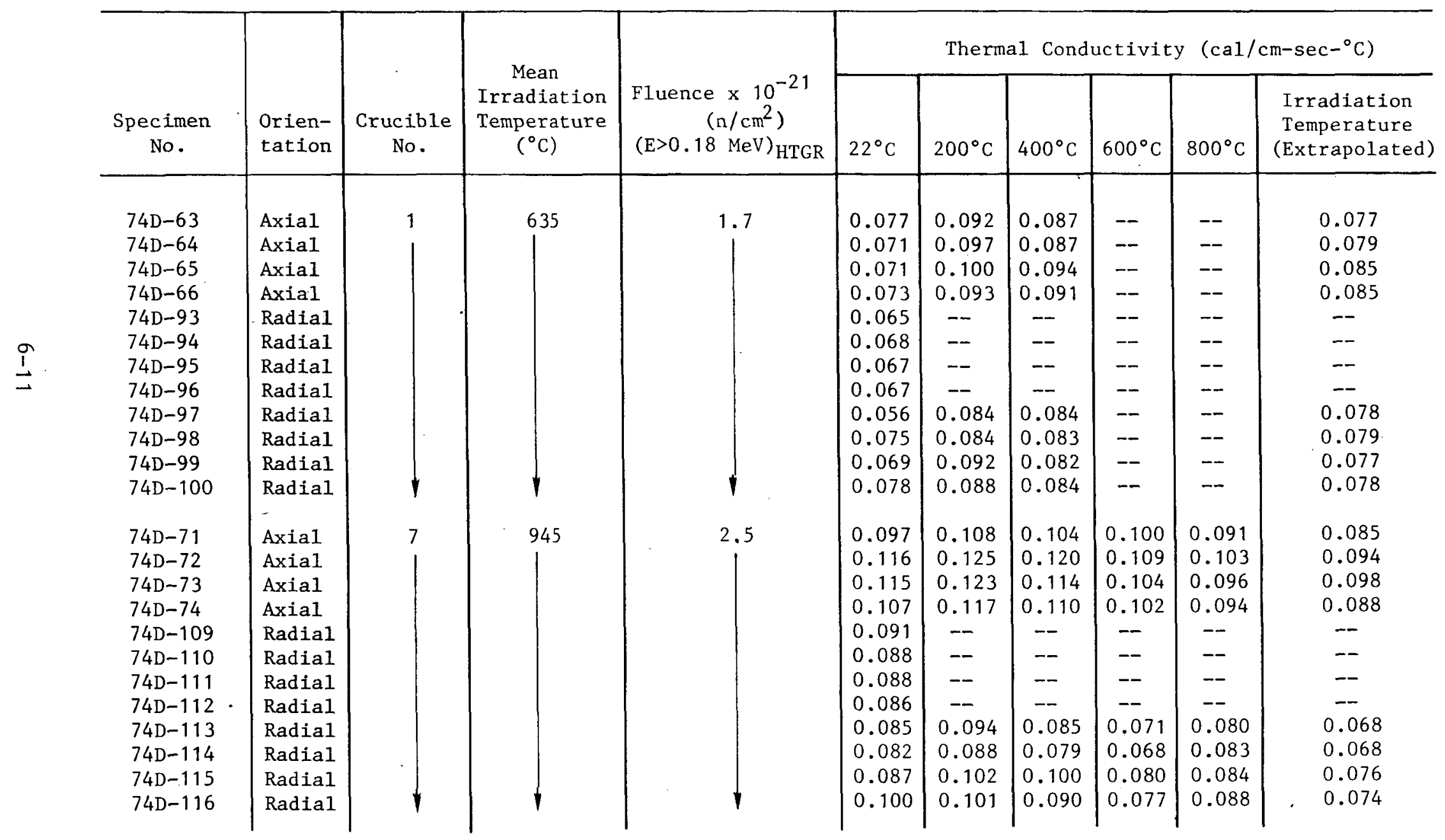


TABLE 6-5 (Continued)

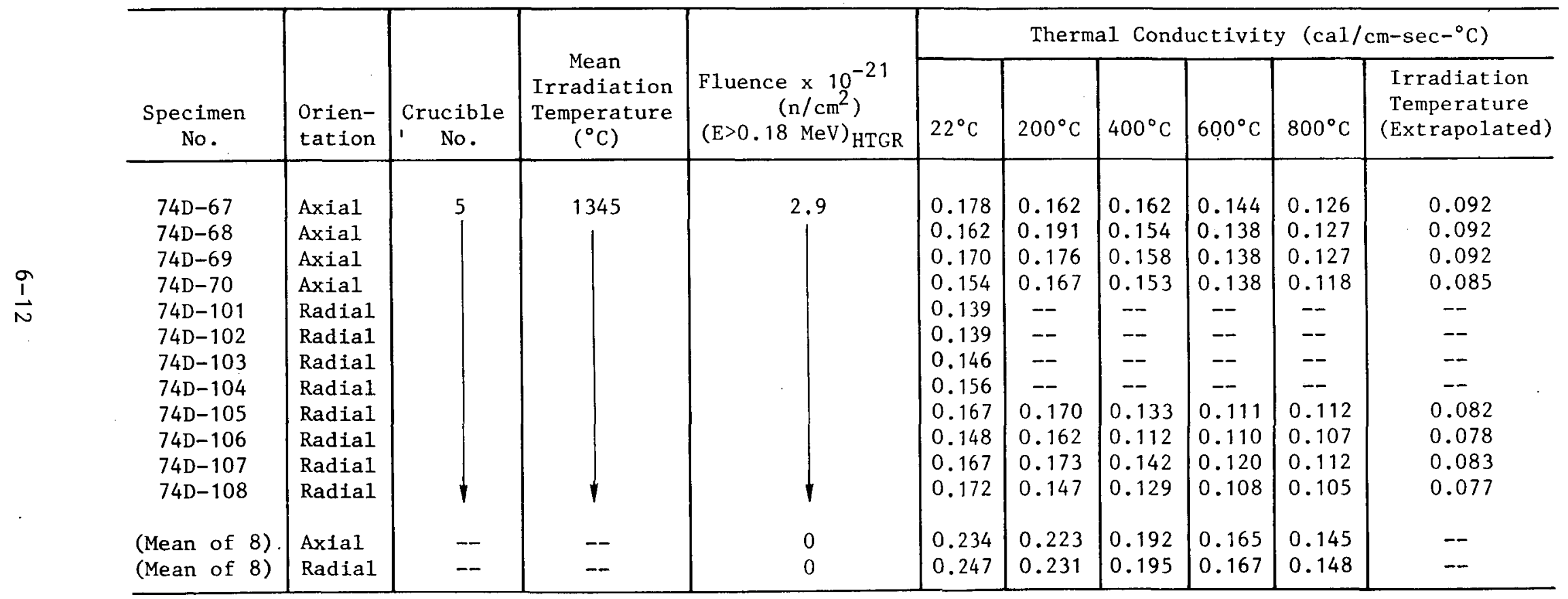


TABLE 6-6

THERMAL CONDUCTIVITY OF H-327 GRAPHITE SPECIMENS IRRADIATED IN CAPSULE OG-2 (A.ll Specimens at Midlength-Edge Location)

\begin{tabular}{|c|c|c|c|c|c|c|c|c|c|c|}
\hline \multirow[b]{2}{*}{$\begin{array}{l}\text { Specimen } \\
\quad \text { No. }\end{array}$} & \multirow[b]{2}{*}{$\begin{array}{l}\text { Orien- } \\
\text { tation }\end{array}$} & \multirow[b]{2}{*}{$\begin{array}{l}\text { Crucible } \\
\text { No. }\end{array}$} & \multirow{2}{*}{$\begin{array}{l}\quad \text { Mean } \\
\text { Irradiation } \\
\text { Temperature } \\
\quad\left({ }^{\circ} \mathrm{C}\right)\end{array}$} & \multirow[b]{2}{*}{$\begin{array}{c}\text { Fluence } \times 10^{-21} \\
\left(\mathrm{n} / \mathrm{cm}^{2}\right) \\
(\mathrm{E}>0.18 \mathrm{MeV})_{\mathrm{HTGR}}\end{array}$} & \multicolumn{6}{|c|}{ Thermal Conductivity $\left(\mathrm{cal} / \mathrm{cm}-\mathrm{sec}-{ }^{\circ} \mathrm{C}\right)$} \\
\hline & & & & & $22^{\circ} \mathrm{C}$ & $200^{\circ} \mathrm{C}$ & $400^{\circ} \mathrm{C}$ & $600^{\circ} \mathrm{C}$ & $800^{\circ} \mathrm{C}$ & $\begin{array}{l}\text { Irradiation } \\
\text { Temperature } \\
\text { (Extrapolated) }\end{array}$ \\
\hline 81 & Axial & 1 & $600-635$ & 4.3 & 0.069 & 0.118 & 0.120 & -- & -- & 0.105 \\
\hline 82 & Axial & & & & 0.086 & 0.108 & 0.109 & -- & -- & 0.095 \\
\hline 1 & Radial & & & & 0.046 & 0.055 & 0.053 & -- & -- & 0.049 \\
\hline 2 & Radial & & & & 0.047 & 0.057 & 0.056 & -- & -- & 0.051 \\
\hline 13 & Radial & 1 & 1 & 1 & 0.052 & 0.060 & 0.064 & -- & -- & 0.056 \\
\hline 83 & Axial & 7 & $900-945$ & 7.1 & 0.074 & 0.087 & 0.076 & 0.077 & 0.077 & 0.072 \\
\hline 84 & Axial & & $900-945$ & & 0.072 & 0.076 & 0.074 & 0.073 & 0.069 & 0.064 \\
\hline $\begin{array}{l}3 \\
4\end{array}$ & Radial & & $875-945$ & & 0.048 & 0.064 & 0.057 & 0.054 & 0.051 & 0.048 \\
\hline $\begin{array}{r}4 \\
32\end{array}$ & Radial & & & & 0.053 & 0.060 & 0.058 & 0.061 & 0.068 & 0.056 \\
\hline & Radial & 1 & 1 & 1 & 0.056 & 0.064 & 0.062 & 0.065 & 0.069 & 0.062 \\
\hline 86 & Axial & 5 & $1225-1345$ & 11.7 & 0.120 & 0.127 & 0.105 & 0.100 & 0.092 & 0.064 \\
\hline 87 & Axia1 & 1 & & & 0.131 & 0.123 & 0.108 & 0.103 & 0.105 & 0.069 \\
\hline 43 & Radial & & & & 0.114 & 0.115 & 0.097 & 0.086 & 0.085 & .0 .060 \\
\hline 44 & Radial & & & & 0.102 & 0.104 & 0.092 & 0.113 & 0.095 & 0.062 \\
\hline 45 & Radial & 1 & 1 & 1 & 0.088 & 0.084 & 0.073 & 0.066 & 0.071 & 0.053 \\
\hline (Mean of 6) & Axial & -- & -- & 0 & 0.450 & 0.367 & 0.293 & 0.240 & 0.194 & -- \\
\hline (Mean of 6) & Radial & -- & -- & 0 & 0.331 & 0.261 & 0.201 & 0.170 & 0.140 & -- \\
\hline
\end{tabular}


O H-451, PERPENDICULAR TO EXTRUSION

$\triangle$ TS-1240, PERPENDICULAR TO EXTRUSION
H-451, PARALLEL TO EXTRUSION

TS-1240, PARALLEL TO EXTRUSION

H-328, PERPENDICULAR TO MOLDING

FAST NEUTRON FLUENCE $\times 10^{-21}\left(\mathrm{~N} / \mathrm{CM}^{2}\right)($ EFFGD)

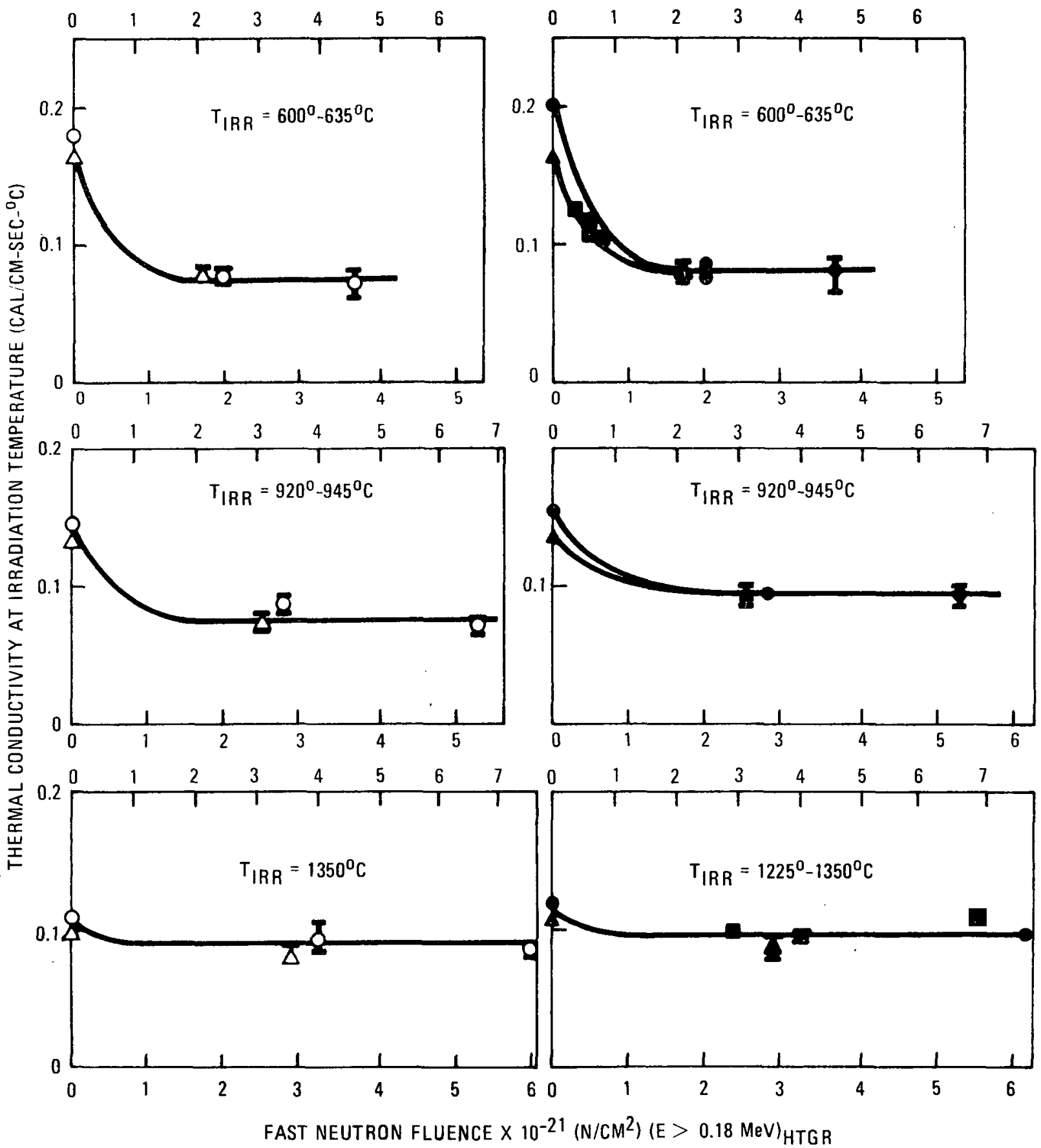

Fig. 6-3. Observed thermal conductivity of $\mathrm{H}-451$ and $\mathrm{TS}-1240$ nearisotropic graphites at irradiation temperature as a function of fast neutron fluence 


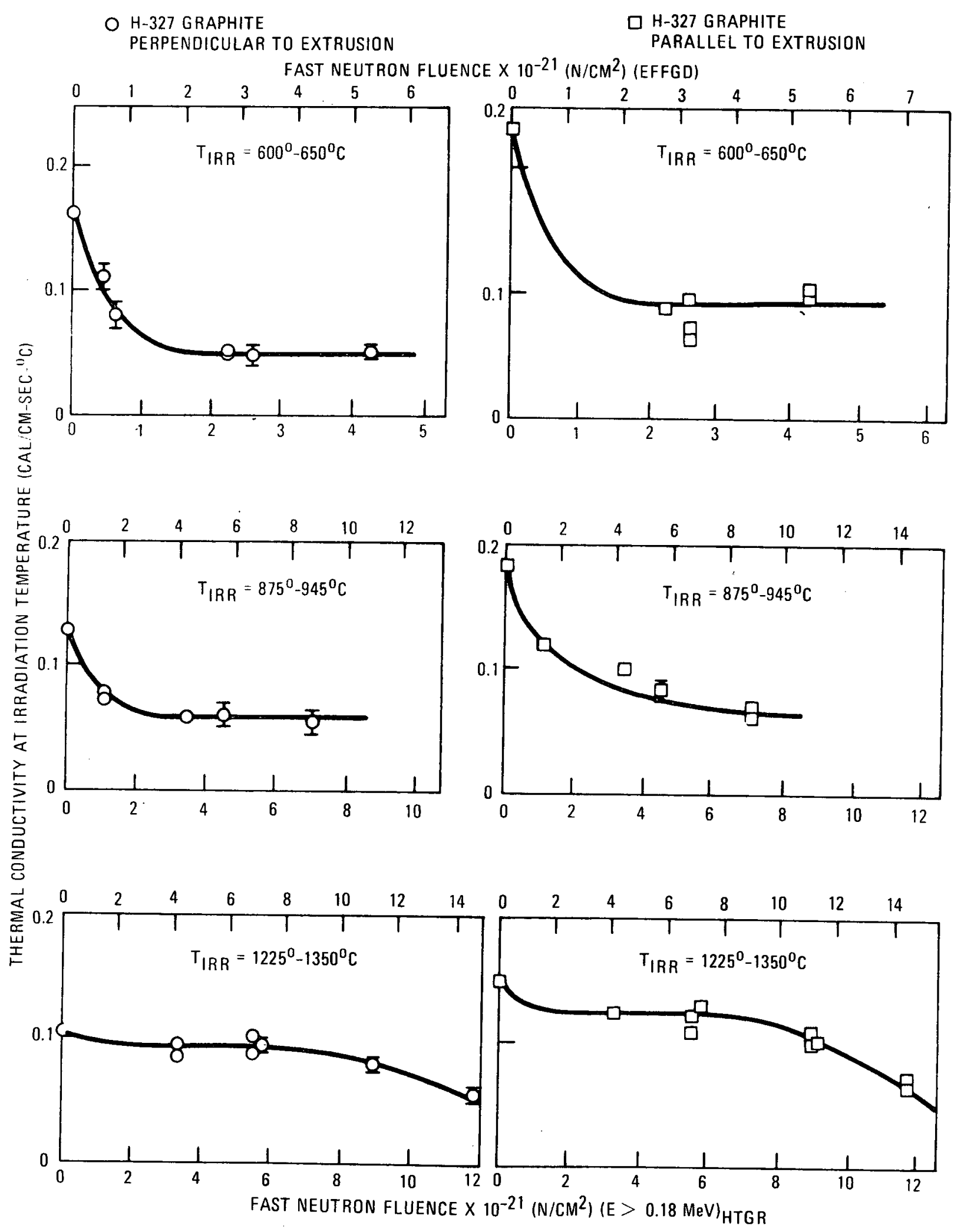

Fig. 6-4. Observed thermal conductivity of H-327 needle coke graphite at irradiation temperature as a function of fast neutron fluence 
where $K_{\text {sat }}(T)$ is the saturation thermal conductivity for irradiation temperature $T, K_{0}$ is the unirradiated thermal conductivity, $\phi t$ is the fast neutron fluence, and $T(T)$ is the time constant for irradiation temperature T. Equation 6-1 may be assumed to hold until volume expansion starts and the second decline in conductivity begins. $\tau(T)$ and $K_{\text {sat }}(T)$ can be stated empirically in terms of the irradiation temperature, $T$ :

$$
\begin{aligned}
\tau(T) & =A+B T, \\
K_{\text {sat }}(T) & =\exp (C+D T) .
\end{aligned}
$$

The experimental data for the room-temperature thermal conductivity - of irradiated $\mathrm{H}-451, \mathrm{TS}-1240$, and $\mathrm{H}-327$ graphites contained in Tables $6-4$ through 6-6 and in Ref. 4 were fitted to Eqs. 6-1 through 6-3 by graphical and least-squares methods to obtain best-fit values for the parameters $A$, $B, C$, and $D$. The new values are listed in Table 6-7. These values can be used to calculate the room-temperature thermal conductivity (cal $/ \mathrm{cm}-\mathrm{sec}-{ }^{\circ} \mathrm{C}$ ) in terms of fluence $\left(10^{21} \mathrm{n} / \mathrm{cm}^{2}\right)(E>0.18 \mathrm{MeV})_{\mathrm{HTGR}}$ and irradiation temperature $\left({ }^{\circ} \mathrm{C}\right)$. A model for calculating the conductivity at other temperatures is described in Ref. 7. The calculated values for conductivity at the irradiation temperature are plotted as functions of fast neutron fluence and irradiation temperature in Figs. $6-5$ and $6-6$ for grades $H-451$ and TS -1240 and grade $\mathrm{H}-327$, respectively. 
TABLE 6-7

THERMAL CONDUCTIVITY PARAMETERS FOR IRRADIATED GRAPHITES

(Eqs. 6-1, 6-2, 6-3) (a)

\begin{tabular}{|c|c|c|c|c|c|}
\hline \multirow[b]{2}{*}{ Grade } & \multirow[b]{2}{*}{ Orientation } & \multicolumn{4}{|c|}{ Value of Parameter } \\
\hline & & $\begin{array}{c}\left(10^{21^{\mathrm{A}}} \mathrm{n} / \mathrm{cm}^{2}\right) \\
(\mathrm{E}>0.18 \mathrm{MeV})_{\mathrm{HTGR}}\end{array}$ & $\left(10^{21} \mathrm{n} / \mathrm{cm}^{2} /{ }^{\circ} \mathrm{C}\right)$ & $\begin{array}{c}\mathrm{C} \\
\text { (Unitless) }\end{array}$ & $\left({ }^{\mathrm{D}} \mathrm{C}^{-1}\right)$ \\
\hline $\left.\begin{array}{l}\mathrm{H}-451 \\
\mathrm{TS}-1240\end{array}\right\}$ & Axial & -0.3059 & $9.58 \times 10^{-4}$ & -3.317 & $1.207 \times 10^{-3}$ \\
\hline $\left.\begin{array}{l}H-451 \\
T S-1240\end{array}\right\}$ & Radial & -0.3059 & $9.58 \times 10^{-4}$ & -3.430 & $1.222 \times 10^{-3}$ \\
\hline $\mathrm{H}-327$ & Axial & -0.3059 & $9.58 \times 10^{-4}$ & -3.429 & $1.305 \times 10^{-3}$ \\
\hline $\mathrm{H}-327$ & Radial & -0.3059 & $9.58 \times 10^{-4}$ & -4.215 & $1.821 \times 10^{-3}$ \\
\hline
\end{tabular}

(a) $\mathrm{K}$ in $\mathrm{cal} / \mathrm{cm}-\mathrm{sec}-{ }^{\circ} \mathrm{C}$. 


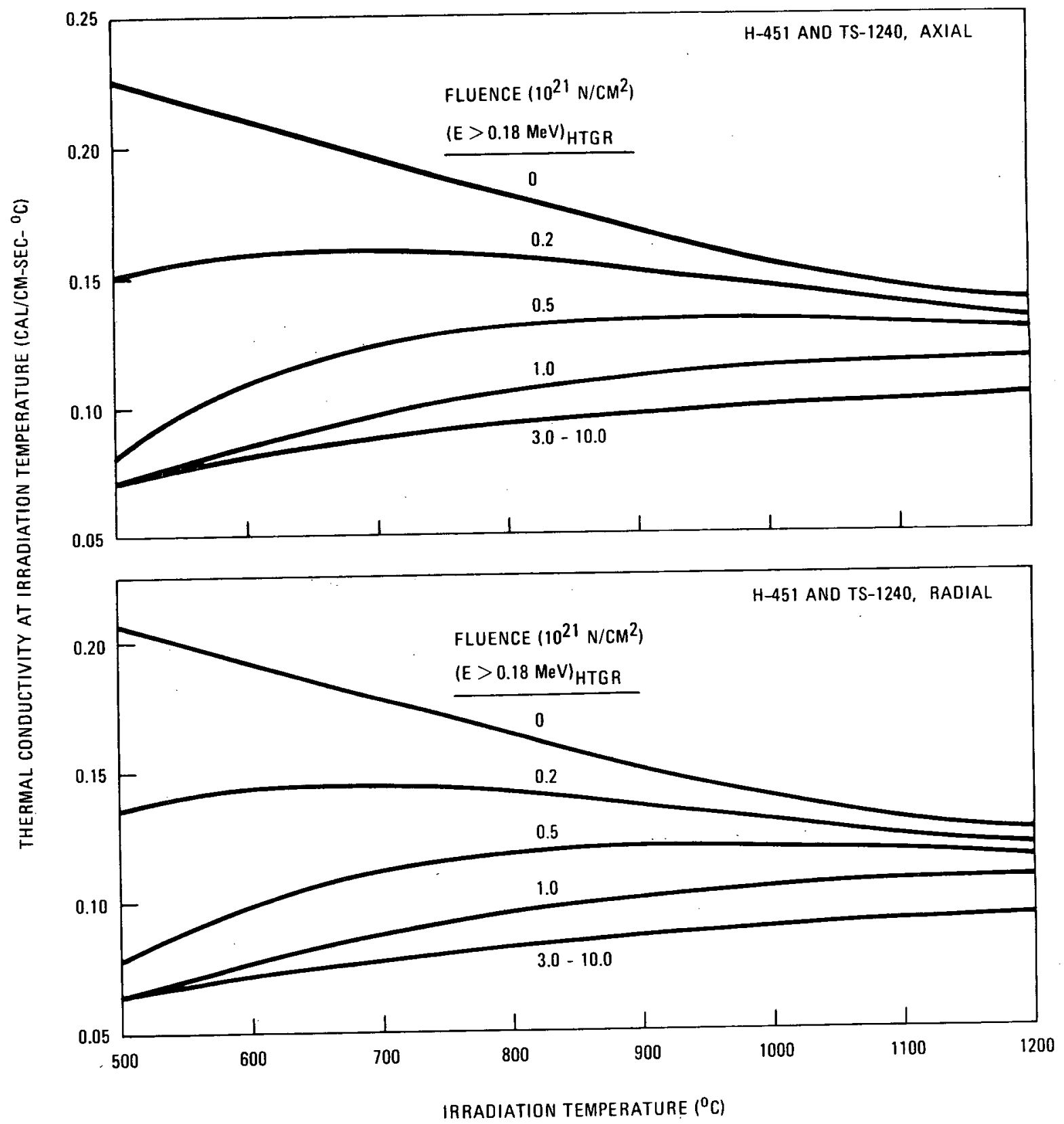

Fig. 6-5. Calculated thermal conductivity of $\mathrm{H}-451$ and TS-1240 nearisotropic graphites at irradiation temperature as a function of irradiation temperature 


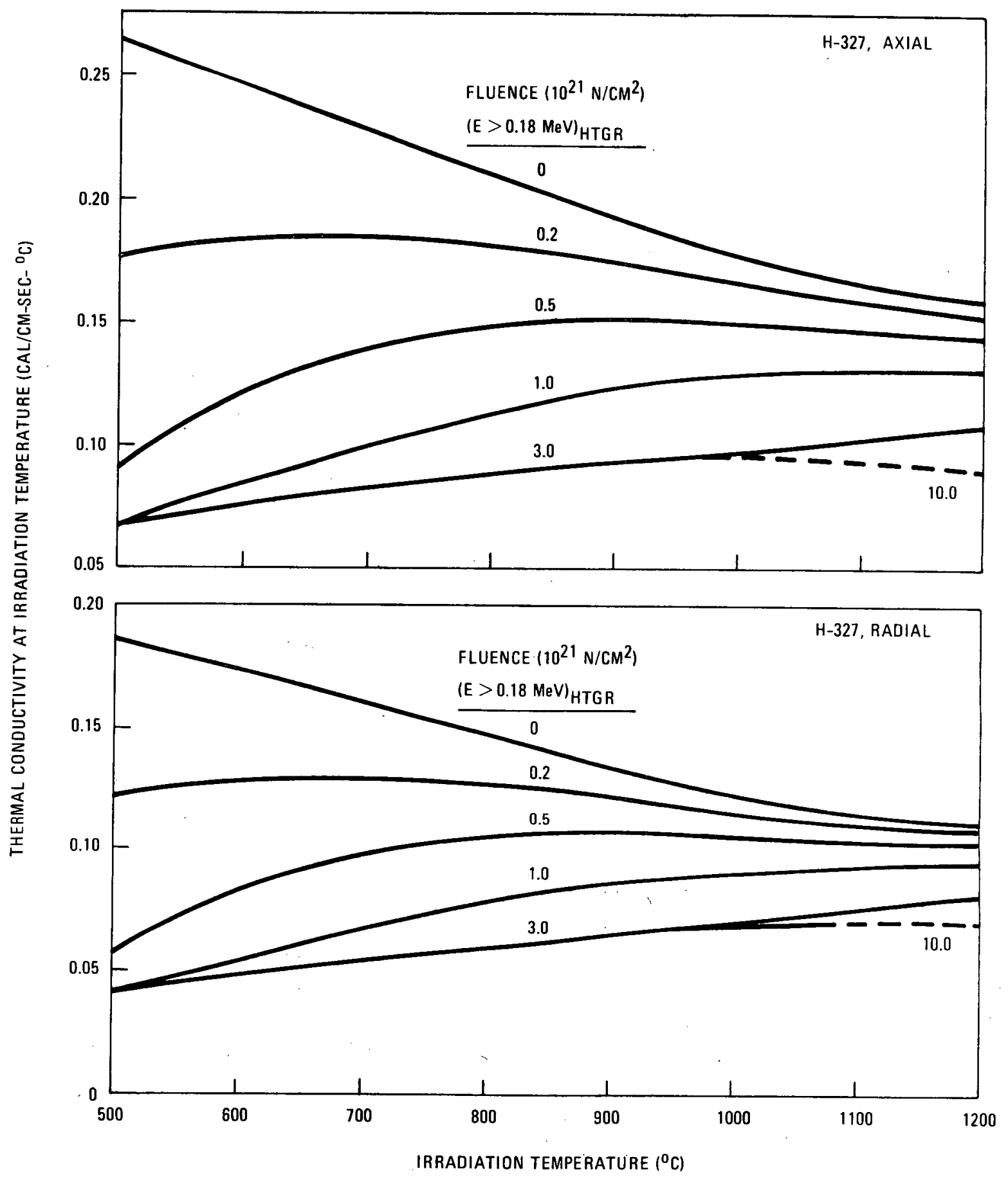

Fig. 6-6. Calculated thermal conductivity of H-327 needle coke graphite at irradiation temperature as a function of irradiation temperature 


\section{EXPERIMENTAL RESULTS: TENSILE PROPERTIES}

Tensile tests were conducted in air at room temperature on 0.25-in.diameter by $0.9-i n .-10 n g$ cylinders of H-451, TS-1240, and H-327 graphite from capsule OG-2. All specimens were from the midlength-center region of the parent log. The specimens of $\mathrm{H}-451$ and some of the H-327 specimens had been irradiated previously in capsule OG-1. An Instron tensile testing machine with a crosshead speed of $0.005 \mathrm{in./min} \mathrm{was} \mathrm{used,} \mathrm{and} \mathrm{strains} \mathrm{were}$ measured with a 0.5-in. gauge length extensometer. The specimens were cemented to metal end-pieces with high-strength epoxy cement, and the load was applied through roller-1ink chains to maintain uniaxial alignment. The specimens were loaded to $1000 \mathrm{psi}$, unloaded to $100 \mathrm{psi}$, and reloaded to failure. Young's modulus was measured from the 100 to 1000 psi reloading part of the curve.

The tensile strengths, strains at failure, Young's moduli, and secant moduli (tensile strength divided by failure strain) are given in Tables 7-1 through 7-3, together with test data for unirradiated companion specimens. The changes in strength and Young's modulus are plotted as functions of fast neutron fluence in Figs. 7-1, 7-2, and 7-3 for H-451, TS-1240, and H-327 graphites, respectively. Figures 7-1 through 7-3 also include data from specimens irradiated in capsule OG-1 (Ref. 4). A summary of tensile data from both capsules appears in Table 7-4.

The increases in Young's' modulus follow the pattern established by Dragon project measurements of the dynamic modulus of irradiated graphites (Ref. 8). During irradiation the modulus rises to a plateau whose level decreases with increasing irradiation temperature. There is an indication of a second increase at higher fluences in the data for $\mathrm{H}-451$ graphite irradiated at $890^{\circ}$ to $970^{\circ} \mathrm{C}$ to $5 \times 10^{21} \mathrm{n} / \mathrm{cm}^{2}$ (Fig. 7-1). The fractional increases in Young's modulus are greater than the increases in dynamic 
TABLE 7-1

TENSILE PROPERTIES OF H-451 GRAPHITE SPECIMENS IRRADIATED IN CAPSULE OG-2

(All Specimens at Midlength-Center Location)

\begin{tabular}{|c|c|c|c|c|c|c|c|c|c|}
\hline $\begin{array}{c}\text { Crucible } \\
\text { No. }\end{array}$ & $\begin{array}{l}\text { Hole } \\
\text { No. }\end{array}$ & $\begin{array}{l}\text { Mean } \\
\text { Irradiation } \\
\text { Temperature } \\
\left({ }^{\circ} \mathrm{C}\right)\end{array}$ & $\begin{array}{c}\text { Fluence } \times 10^{-21} \\
\left(\mathrm{n} / \mathrm{cm}^{2}\right) \\
(E>0,18 \mathrm{MeV})_{\text {HTGR }}\end{array}$ & $\begin{array}{l}\text { Orien- } \\
\text { tation }\end{array}$ & $\begin{array}{c}\text { Specimen } \\
\text { No. }\end{array}$ & $\begin{array}{l}\text { U1timate } \\
\text { Tensile } \\
\text { Strength } \\
\text { (psi) }\end{array}$ & $\begin{array}{l}\text { Strain at } \\
\text { Failure } \\
\quad(\%)\end{array}$ & $\begin{array}{l}\text { Young's } \\
\text { Modulus } \\
\left(10^{6} \text { psi) }\right.\end{array}$ & $\begin{array}{c}\text { Secant } \\
\text { Modulus } \\
\left(10^{6} \text { psi) }\right.\end{array}$ \\
\hline 10 & $\begin{array}{l}24 \\
24 \\
25 \\
25 \\
26 \\
26 \\
27 \\
27\end{array}$ & 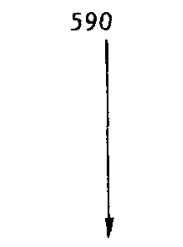 & $i^{2.6}$ & $\mid$ & $\begin{array}{l}\mathrm{T}-173 \\
\mathrm{~T}-174 \\
\mathrm{~T}-175 \\
\mathrm{~T}-176 \\
\mathrm{~T}-177 \\
\mathrm{~T}-178 \\
\mathrm{~T}-179 \\
\mathrm{~T}-180\end{array}$ & $\begin{array}{l}2532 \\
2589 \\
2326 \\
1775 \\
2624 \\
2861 \\
2641 \\
2496\end{array}$ & $\begin{array}{l}0.110 \\
0.138 \\
0.090 \\
0.097 \\
0.121 \\
0.123 \\
0.134 \\
0.117\end{array}$ & $\begin{array}{l}2.61 \\
2.35 \\
2.73 \\
2.21 \\
2.58 \\
2.84 \\
2.51 \\
2.50\end{array}$ & $\begin{array}{l}2.30 \\
1.88 \\
2.58 \\
1.83 \\
2.17 \\
2.33 \\
1.97 \\
2.13\end{array}$ \\
\hline & & & & & $\begin{array}{l}\text { Mean } \\
\text { Std Dev }\end{array}$ & $\begin{array}{l}2481 \\
\pm 322\end{array}$ & $\begin{array}{r}0.116 \\
\pm 0.017\end{array}$ & $\begin{array}{r}2.54 \\
\pm 0.20\end{array}$ & $\begin{array}{r}2.15 \\
\pm 0.25\end{array}$ \\
\hline 7 & $\begin{array}{l}10 \\
10 \\
22 \\
22 \\
23 \\
23 \\
24 \\
24\end{array}$ & $\begin{array}{l}950-970 \\
950-970 \\
890-920\end{array}$ & $i^{5.3}$ & $\mid$ & $\begin{array}{l}\mathrm{T}-155 \\
\mathrm{~T}-156 \\
\mathrm{~T}-157 \\
\mathrm{~T}-158 \\
\mathrm{~T}-159 \\
\mathrm{~T}-160 \\
\mathrm{~T}-161 \\
\mathrm{~T}-162\end{array}$ & $\begin{array}{l}2240 \\
2822 \\
3027 \\
2877 \\
2857 \\
3004 \\
2550 \\
2835 \\
\end{array}$ & $\begin{array}{l}0.105 \\
0.130 \\
0.136 \\
0.127 \\
0.125 \\
0.150 \\
0.122 \\
0.145 \\
\end{array}$ & $\begin{array}{l}2.50 \\
2.70 \\
2.59 \\
2.74 \\
2.61 \\
2.28 \\
2.52 \\
2.43 \\
\end{array}$ & $\begin{array}{l}2.13 \\
2.17 \\
2.23 \\
2.27 \\
2.29 \\
2.00 \\
2.09 \\
1.96 \\
\end{array}$ \\
\hline & & & & & Mean & 2777 & 0.130 & 2.55 & 2.14 \\
\hline & & & & & Std Dev & \pm 261 & \pm 0.014 & \pm 0.15 & \pm 0.12 \\
\hline-- & -- & -- & 0 & Axial & $\begin{array}{l}\mathrm{T}-181 \\
\mathrm{~T}-182 \\
\mathrm{~T}-183 \\
\mathrm{~T}-184 \\
\mathrm{~T}-185 \\
\mathrm{~T}-186 \\
\mathrm{~T}-187 \\
\mathrm{~T}-188 \\
\mathrm{~T}-190 \\
\end{array}$ & $\begin{array}{l}1446 \\
1630 \\
1650 \\
1589 \\
1548 \\
1731 \\
1243 \\
1609 \\
1630 \\
\end{array}$ & $\begin{array}{l}0.130 \\
0.230 \\
0.212 \\
0.169 \\
0.152 \\
0.211 \\
0.182 \\
0.187 \\
0.184 \\
\end{array}$ & $\begin{array}{l}1.22 \\
1.02 \\
1.15 \\
1.17 \\
1.20 \\
1.23 \\
0.95 \\
1.17 \\
1.17 \\
\end{array}$ & $\begin{array}{l}1.11 \\
0.71 \\
0.78 \\
0.94 \\
1.02 \\
0.82 \\
0.68 \\
0.86 \\
0.89 \\
\end{array}$ \\
\hline & & & & & $\begin{array}{l}\text { Mean } \\
\text { Std Dev }\end{array}$ & $\begin{array}{r}1564 \\
\pm 135\end{array}$ & $\begin{array}{r}0.184 \\
\pm 0.029\end{array}$ & $\begin{array}{r}1.14 \\
\pm 0.09\end{array}$ & $\begin{array}{r}0.87 \\
\pm 0.13\end{array}$ \\
\hline-- & -- & -- & $\begin{array}{l}0 \\
1\end{array}$ & Radial & $\begin{array}{r}\mathrm{T}-219 \\
\mathrm{~T}-220 \\
\mathrm{~T}-221 \\
\mathrm{~T}-222 \\
\mathrm{~T}-223 \\
\mathrm{~T}-224 \\
\mathrm{~T}-225 \\
\mathrm{~T}-226 \\
\mathrm{~T}-227 \\
\mathrm{~T}-228 \\
1 \\
2 \\
3 \\
5 \\
6\end{array}$ & $\begin{array}{l}1589 \\
1751 \\
1874 \\
1731 \\
1650 \\
2037 \\
1283 \\
1609 \\
1853 \\
1344 \\
1446 \\
1508 \\
2220 \\
1609 \\
1562 \\
\end{array}$ & $\begin{array}{l}0.210 \\
0.215 \\
0.252 \\
0.223 \\
0.210 \\
0.267 \\
0.172 \\
0.181 \\
0.241 \\
0.147 \\
0.227 \\
0.242 \\
0.342 \\
0.219 \\
0.214 \\
\end{array}$ & $\begin{array}{l}0.97 \\
0.98 \\
1.02 \\
1.02 \\
0.94 \\
1.02 \\
0.97 \\
1.12 \\
1.06 \\
1.02 \\
0.92 \\
0.91 \\
1.03 \\
0.99 \\
1.08 \\
\end{array}$ & $\begin{array}{l}0.76 \\
0.81 \\
0.74 \\
0.77 \\
0.79 \\
0.76 \\
0.75 \\
0.89 \\
0.77 \\
0.91 \\
0.64 \\
0.62 \\
0.65 \\
0.73 \\
0.73 \\
\end{array}$ \\
\hline & & & & & $\begin{array}{l}\text { Mean } \\
\text { Std Dev }\end{array}$ & $\begin{array}{r}1671 \\
\pm 242\end{array}$ & $\begin{array}{r}0.224 \\
\pm 0.043\end{array}$ & $\begin{array}{r}1.00 \\
\pm 0.05\end{array}$ & $\begin{array}{r}0.75 \\
\pm 0.08\end{array}$ \\
\hline
\end{tabular}


TABLE 7-2

TENSTLE PROPERTIES OF TS-1240 GRAPHITE SPECIMENS IRRADIATED IN CAPSULE OG-2 (All Specimens at Midlength-Center Location)

\begin{tabular}{|c|c|c|c|c|c|c|c|c|c|}
\hline $\begin{array}{c}\text { Crucible } \\
\text { No. }\end{array}$ & $\begin{array}{l}\text { Hole } \\
\text { No. }\end{array}$ & $\begin{array}{l}\text { Mean } \\
\text { Irradiation } \\
\text { Temperature } \\
\quad\left({ }^{\circ} \mathrm{C}\right)\end{array}$ & $\begin{array}{c}\text { Fluence } \times 10^{-21} \\
\left(\mathrm{n} / \mathrm{cm}^{2}\right) \\
(\mathrm{E}>0.18 \mathrm{MeV})_{\mathrm{HTGR}}\end{array}$ & $\begin{array}{l}\text { Orien- } \\
\text { tation }\end{array}$ & $\begin{array}{c}\text { Specimen } \\
\text { No. }\end{array}$ & $\begin{array}{l}\text { Ultimate } \\
\text { Tensile } \\
\text { Strength } \\
\quad \text { (psi) }\end{array}$ & $\begin{array}{c}\text { Strain At } \\
\text { Failure } \\
(\%)\end{array}$ & $\begin{array}{l}\text { Young's } \\
\text { Modulus } \\
\left(10^{6} \text { psi) }\right.\end{array}$ & $\begin{array}{l}\text { Secant } \\
\text { Modulus } \\
\left(10^{6} \text { psi) }\right.\end{array}$ \\
\hline 1 & $\begin{array}{l}10 \\
10 \\
11 \\
11 \\
12 \\
12 \\
13 \\
13 \\
14 \\
14\end{array}$ & $\left.\right|^{620}$ & 1.2 & 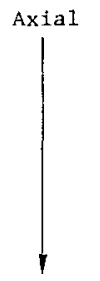 & $\begin{array}{l}\text { T } 40105 \\
T 40107 \\
T 40109 \\
\text { T } 40111 \\
T 40113 \\
T 40115 \\
T 40117 \\
T 40118 \\
T 40119 \\
T 40120\end{array}$ & $\begin{array}{l}1940 \\
1426 \\
1104 \\
1492 \\
2221 \\
2528 \\
2202 \\
1653 \\
1629 \\
2246\end{array}$ & $\begin{array}{l}0.104 \\
0.116 \\
0.100 \\
0.096 \\
0.156 \\
0.148 \\
0.133 \\
-- \\
0.114 \\
0.167\end{array}$ & $\begin{array}{l}2.16 \\
1.34 \\
1.22 \\
1.99 \\
1.64 \\
1.93 \\
2.09 \\
1.54 \\
1.70 \\
1.57\end{array}$ & $\begin{array}{l}1.87 \\
1.23 \\
1.10 \\
1.55 \\
1.42 \\
1.71 \\
1.66 \\
-. \\
1.43 \\
1.34\end{array}$ \\
\hline & & & & & $\begin{array}{c}\text { Mean } \\
\text { Std Dev }\end{array}$ & $\begin{array}{r}1844 \\
\pm 452\end{array}$ & $\begin{array}{r}0.126 \\
\pm 0.026\end{array}$ & $\begin{array}{r}1.72 \\
\pm 0.32\end{array}$ & $\begin{array}{r}1.48 \\
+0.24\end{array}$ \\
\hline 1 & $\begin{array}{l}28 \\
28 \\
29 \\
29 \\
30 \\
30 \\
31 \\
31 \\
32 \\
32\end{array}$ & 765 & $i^{1.7}$ & Axial & $\begin{array}{l}\text { T40069 } \\
\text { T40071 } \\
T 40073 \\
T 40075 \\
T 40077 \\
T 40079 \\
T 40081 \\
T 40083 \\
T 40085 \\
T 40087\end{array}$ & $\begin{array}{l}1204 \\
1981 \\
2270 \\
1964 \\
2354 \\
1822 \\
2309 \\
2516 \\
2385 \\
1895\end{array}$ & $\begin{array}{l}0.089 \\
0.140 \\
0.182 \\
0.127 \\
0.150 \\
0.122 \\
0.144 \\
0.163 \\
0.153 \\
0.143\end{array}$ & $\begin{array}{l}1.56 \\
1.62 \\
1.47 \\
1.78 \\
2.05 \\
1.67 \\
1.84 \\
1.81 \\
1.85 \\
1.58\end{array}$ & $\begin{array}{l}1.35 \\
1.42 \\
1.25 \\
1.55 \\
1.57 \\
1.49 \\
1.60 \\
1.54 \\
1.56 \\
1.32\end{array}$ \\
\hline & & . & & & $\begin{array}{l}\text { Mean } \\
\text { Std Dev }\end{array}$ & $\begin{array}{l}2070 \\
\pm 385\end{array}$ & $\begin{array}{r}0.141 \\
\pm 0.025\end{array}$ & $\begin{array}{r}1.72 \\
\pm 0.17\end{array}$ & $\begin{array}{r}1.47 \\
\pm 0.12\end{array}$ \\
\hline 9 & $\begin{array}{l}12 \\
12 \\
13 \\
13 \\
14 \\
14 \\
15 \\
15 \\
16 \\
16\end{array}$ & $\mathfrak{l}^{805}$ & $\stackrel{1.7}{1}$ & $\underset{1}{\text { Radial }}$ & $\begin{array}{l}\mathrm{T} 40129 \\
\mathrm{~T} 40131 \\
\mathrm{~T} 40133 \\
\mathrm{~T} 40135 \\
\mathrm{~T} 40137 \\
\mathrm{~T} 40139 \\
\mathrm{~T} 40141 \\
\mathrm{~T} 40143 \\
\mathrm{~T} 40145 \\
\mathrm{~T} 40147(\mathrm{a})\end{array}$ & $\begin{array}{l}2163 \\
1940 \\
1816 \\
1899 \\
2180 \\
1181 \\
2302 \\
2100 \\
1979 \\
1084(a)\end{array}$ & $\begin{array}{l}0.140 \\
0.113 \\
0.131 \\
0.168 \\
0.166 \\
0.138 \\
0.174 \\
0.144 \\
0.160 \\
0.194(a)\end{array}$ & $\begin{array}{l}1.74 \\
1.88 \\
1.56 \\
1.33 \\
1.48 \\
0.99 \\
1.54 \\
1.62 \\
1.38 \\
0.66(a)\end{array}$ & $\begin{array}{l}1.55 \\
1.72 \\
1.39 \\
1.13 \\
1.31 \\
0.86 \\
1.32 \\
1.46 \\
1.24 \\
0.56(\mathrm{a})\end{array}$ \\
\hline & & & & & $\begin{array}{l}\text { Mean } \\
\text { Std Dev }\end{array}$ & $\begin{array}{r}1951 \\
\pm 327\end{array}$ & $\begin{array}{r}0.148 \\
\pm 0.020\end{array}$ & $\begin{array}{r}1.50 \\
\pm 0.26\end{array}$ & $\begin{array}{r}1.33 \\
\pm 0.25\end{array}$ \\
\hline $\begin{array}{l}7 \\
1\end{array}$ & $\begin{array}{l}25 \\
25 \\
26 \\
26 \\
27 \\
27 \\
28 \\
28\end{array}$ & 920 & $j^{2.5}$ & Axial & $\begin{array}{l}\mathrm{T} 40053 \\
\mathrm{~T} 40055 \\
\mathrm{~T} 40057 \\
\mathrm{~T} 40059 \\
\mathrm{~T} 40061 \\
\mathrm{~T} 40063 \\
\mathrm{~T} 40065 \\
\mathrm{~T} 40067\end{array}$ & $\begin{array}{l}2330 \\
1696 \\
2385 \\
2467 \\
1918 \\
2042 \\
2122 \\
2023 \\
\end{array}$ & $\begin{array}{l}0.162 \\
0.136 \\
0.178 \\
0.166 \\
0.138 \\
0.144 \\
0.151 \\
0.172\end{array}$ & $\begin{array}{l}1.72 \\
1.63 \\
1.55 \\
1.83 \\
1.50 \\
1.81 \\
1.74 \\
1.52\end{array}$ & $\begin{array}{l}1.44 \\
1.25 \\
1.34 \\
1.49 \\
1.39 \\
1.42 \\
1.41 \\
1.18\end{array}$ \\
\hline & & & - & & $\begin{array}{l}\text { Mean } \\
\text { Std Dev }\end{array}$ & $\begin{array}{l}2123 \\
\pm 259\end{array}$ & $\begin{array}{r}0.156 \\
\pm 0.016\end{array}$ & $\begin{array}{r}1.66 \\
\pm 0.13\end{array}$ & $\begin{array}{r}1.37 \\
\pm 0.10\end{array}$ \\
\hline $\int^{4}$ & $\begin{array}{l}28 \\
28 \\
29 \\
29 \\
30 \\
30 \\
31 \\
31 \\
32 \\
32\end{array}$ & 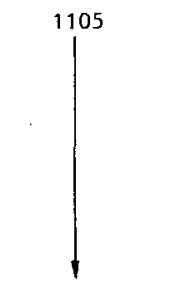 & $\begin{array}{c}2.9 \\
1\end{array}$ & $\stackrel{\text { Axial }}{\mid}$ & $\begin{array}{l}\mathrm{T} 400017 \\
\mathrm{~T} 40019 \\
\mathrm{~T} 40021 \\
\mathrm{~T} 40023 \\
\mathrm{~T} 40025 \\
\mathrm{~T} 40027 \\
\mathrm{~T} 40029 \\
\mathrm{~T} 40031 \\
\mathrm{~T} 40033 \\
\mathrm{~T} 40035\end{array}$ & $\begin{array}{l}2485 \\
1438 \\
1729 \\
2798 \\
2205 \\
2328 \\
1235 \\
1706 \\
1214 \\
1855 \\
\end{array}$ & $\begin{array}{l}0.197 \\
0.114 \\
0.125 \\
0.220 \\
0.144 \\
0.153 \\
0.098 \\
0.154 \\
-.- \\
0.106 \\
\end{array}$ & $\begin{array}{l}1.64 \\
1.56 \\
1.70 \\
1.56 \\
1.92 \\
1.91 \\
1.48 \\
1.40 \\
1.70 \\
2.06 \\
\end{array}$ & $\begin{array}{l}1.26 \\
1.26 \\
1.38 \\
1.27 \\
1.53 \\
1.52 \\
1.26 \\
1.11 \\
-.- \\
1.75 \\
\end{array}$ \\
\hline & & & & & $\begin{array}{c}\text { Mean } \\
\text { Std Dev }\end{array}$ & $\begin{array}{l}1899 \\
\pm 540\end{array}$ & $\begin{array}{r}0.146 \\
\pm 0.041\end{array}$ & $\begin{array}{r}1.69 \\
\pm 0.21\end{array}$ & $\begin{array}{r}1.37 \\
\pm 0.20\end{array}$ \\
\hline
\end{tabular}

(a) Specimen flawed; excluded from averages. 
TABLE 7-2 (Continued)

\begin{tabular}{|c|c|c|c|c|c|c|c|c|c|}
\hline $\begin{array}{c}\text { Crucible } \\
\text { No. }\end{array}$ & $\begin{array}{l}\text { Hole } \\
\text { No. }\end{array}$ & $\begin{array}{l}\text { Mean } \\
\text { Irradiation } \\
\text { Temperature } \\
\qquad\left({ }^{\circ} \mathrm{C}\right)\end{array}$ & $\begin{array}{c}\text { Fluence } \times 10^{-21} \\
\left(\mathrm{n} / \mathrm{cm}^{2}\right) \\
(\mathrm{E}>0.18 \mathrm{MeV})_{\text {HTGR }}\end{array}$ & $\begin{array}{l}\text { Orien- } \\
\text { tation }\end{array}$ & $\begin{array}{c}\text { Specimen } \\
\text { No. }\end{array}$ & $\begin{array}{l}\text { Ultimate } \\
\text { Tensile } \\
\text { Strength } \\
\quad \text { (psi) }\end{array}$ & $\begin{array}{c}\text { Strain At } \\
\text { Failure } \\
(\%)\end{array}$ & $\begin{array}{l}\text { Young's } \\
\text { Modulus } \\
\left(10^{6} \text { psi) }\right.\end{array}$ & $\begin{array}{l}\text { Secant } \\
\text { Modulus } \\
\left(10^{6} \text { ps } 1\right)\end{array}$ \\
\hline-- & \multirow[t]{2}{*}{--} & \multirow[t]{2}{*}{--} & \multirow[t]{2}{*}{0} & \multirow[t]{2}{*}{ Axial } & $\begin{array}{r}3 \mathrm{~A}-6 \mathrm{~B} \\
7 \mathrm{~B} \\
12 \mathrm{~B} \\
13 \mathrm{~B} \\
18 \mathrm{~B} \\
19 \mathrm{~B} \\
24 \mathrm{~B} \\
25 \mathrm{~B} \\
27 \mathrm{~B} \\
28 \mathrm{~B} \\
3 \mathrm{~B}-6 \mathrm{~B} \\
7 \mathrm{~B} \\
12 \mathrm{~B} \\
13 \mathrm{~B} \\
18 \mathrm{~B} \\
19 \mathrm{~B} \\
24 \mathrm{~B} \\
25 \mathrm{~B} \\
27 \mathrm{~B} \\
28 \mathrm{~B}\end{array}$ & $\begin{array}{l}1427 \\
1711 \\
2015 \\
1896 \\
1469 \\
1406 \\
1796 \\
1691 \\
1449 \\
1672 \\
1976 \\
1670 \\
1438 \\
1141 \\
1080 \\
1283 \\
1487 \\
1569 \\
1507 \\
1731\end{array}$ & $\begin{array}{l}0.210 \\
0.270 \\
0.310 \\
0.320 \\
0.200 \\
0.285 \\
0.275 \\
0.250 \\
0.245 \\
0.265 \\
0.323 \\
0.245 \\
0.185 \\
0.145 \\
0.240 \\
0.190 \\
0.215 \\
0.295 \\
0.275 \\
0.335\end{array}$ & $\begin{array}{l}0.90 \\
0.95 \\
1.00 \\
0.90 \\
0.86 \\
0.69 \\
0.95 \\
1.00 \\
0.86 \\
0.90 \\
1.00 \\
1.00 \\
1.00 \\
0.95 \\
0.60 \\
0.86 \\
0.88 \\
0.90 \\
0.86 \\
0.86\end{array}$ & $\begin{array}{l}0.68 \\
0.63 \\
0.65 \\
0.59 \\
0.73 \\
0.49 \\
0.65 \\
0.68 \\
0.59 \\
0.63 \\
0.61 \\
0.68 \\
0.78 \\
0.79 \\
0.45 \\
0.68 \\
0.69 \\
0.53 \\
0.55 \\
0.52\end{array}$ \\
\hline$\cdot$ & & & & & $\begin{array}{c}\text { Mean } \\
\text { Std Dev }\end{array}$ & $\begin{array}{l}1571 \\
\pm 252\end{array}$ & $\begin{array}{r}0.254 \\
\pm 0.051\end{array}$ & $\begin{array}{r}0.90 \\
+0.10\end{array}$ & $\begin{array}{r}0.63 \\
\pm 0.09\end{array}$ \\
\hline-- & \multirow[t]{3}{*}{--} & \multirow[t]{3}{*}{--} & \multirow[t]{3}{*}{0} & \multirow[t]{3}{*}{ Radial } & $\begin{array}{r}3 \mathrm{~A}-36 \mathrm{~B} \\
37 \mathrm{~B} \\
42 \mathrm{~B} \\
43 \mathrm{~B} \\
48 \mathrm{~B} \\
49 \mathrm{~B} \\
54 \mathrm{~B} \\
55 \mathrm{~B} \\
60 \mathrm{~A} \\
60 \mathrm{~B} \\
3 \mathrm{~B}-36 \mathrm{~B} \\
37 \mathrm{~B} \\
42 \mathrm{~B} \\
43 \mathrm{~B} \\
48 \mathrm{~B} \\
49 \mathrm{~B} \\
54 \mathrm{~B} \\
55 \mathrm{~B} \\
60 \mathrm{~A} \\
60 \mathrm{~B}\end{array}$ & $\begin{array}{r}957 \\
1428 \\
1367 \\
1266 \\
1388 \\
1120 \\
1450 \\
1531 \\
2400 \\
1170 \\
1448 \\
958 \\
1428 \\
1287 \\
1594 \\
1491 \\
1384 \\
1428 \\
1428 \\
1144\end{array}$ & $\begin{array}{l}0.140 \\
0.220 \\
0.205 \\
0.300 \\
0.245 \\
0.130 \\
0.210 \\
0.250 \\
0.350 \\
0.175 \\
0.330 \\
0.165 \\
0.210 \\
0.195 \\
0.252 \\
0.255 \\
0.205 \\
0.233 \\
0.295 \\
0.255\end{array}$ & $\begin{array}{l}-- \\
0.95 \\
1.00 \\
0.78 \\
0.72 \\
1.00 \\
0.90 \\
0.95 \\
1.06 \\
0.90 \\
0.75 \\
-- \\
0.95 \\
0.88 \\
0.90 \\
0.95 \\
1.00 \\
0.91 \\
0.83 \\
0.68\end{array}$ & $\begin{array}{l}0.68 \\
0.65 \\
0.67 \\
0.42 \\
0.57 \\
0.86 \\
0.69 \\
0.61 \\
0.69 \\
0.67 \\
0.44 \\
0.58 \\
0.68 \\
0.66 \\
0.63 \\
0.58 \\
0.68 \\
0.61 \\
0.48 \\
0.45\end{array}$ \\
\hline & & & & & & 1383 & 0.231 & 0.90 & 0.62 \\
\hline & & & & & Std Dev & \pm 298 & \pm 0.058 & \pm 0.11 & \pm 0.11 \\
\hline
\end{tabular}


TABLE 7-3

TENSLLE PROPERTIES OF H-327 GRAPHITE SPECIMENS IRRADIATED IN CAPSULE OG-2

(Al1 Specimens at Midlength-Center Location)

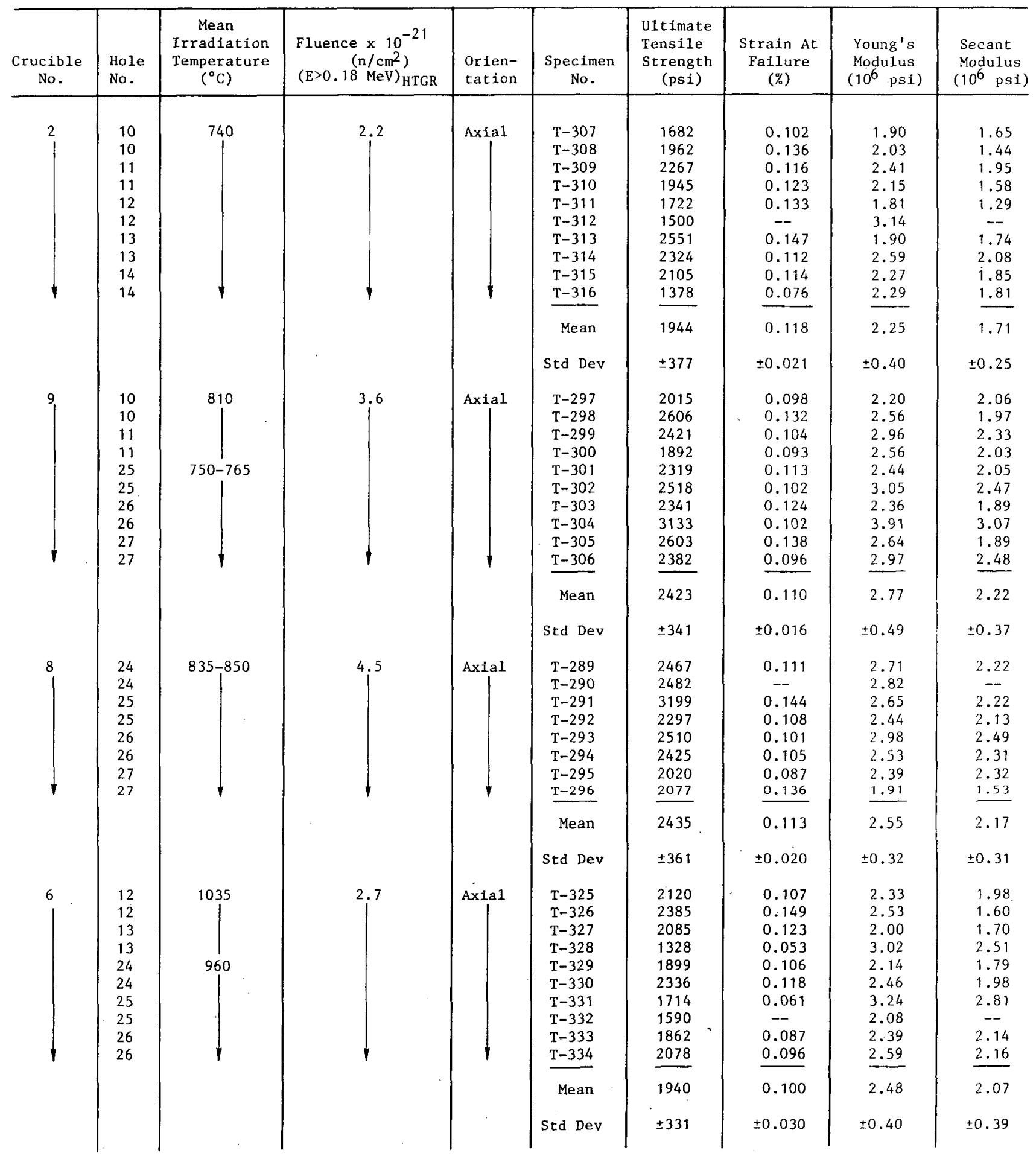


TABLE 7-3 (Continued)

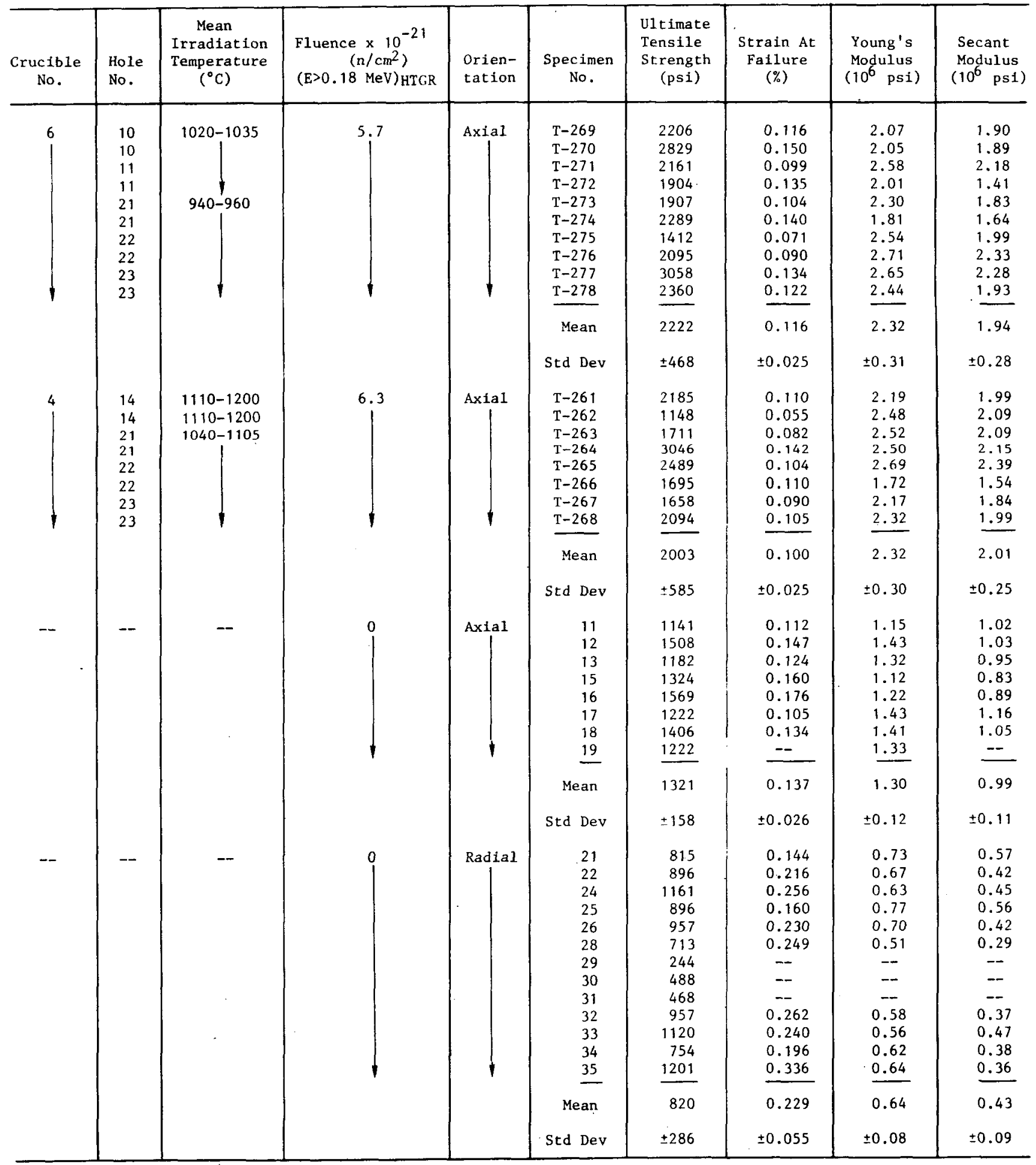


FAST NEUTRON FLUENCE $\times 10^{-21}\left(\mathrm{~N} / \mathrm{CM}^{2}\right)$ (EFFGD)
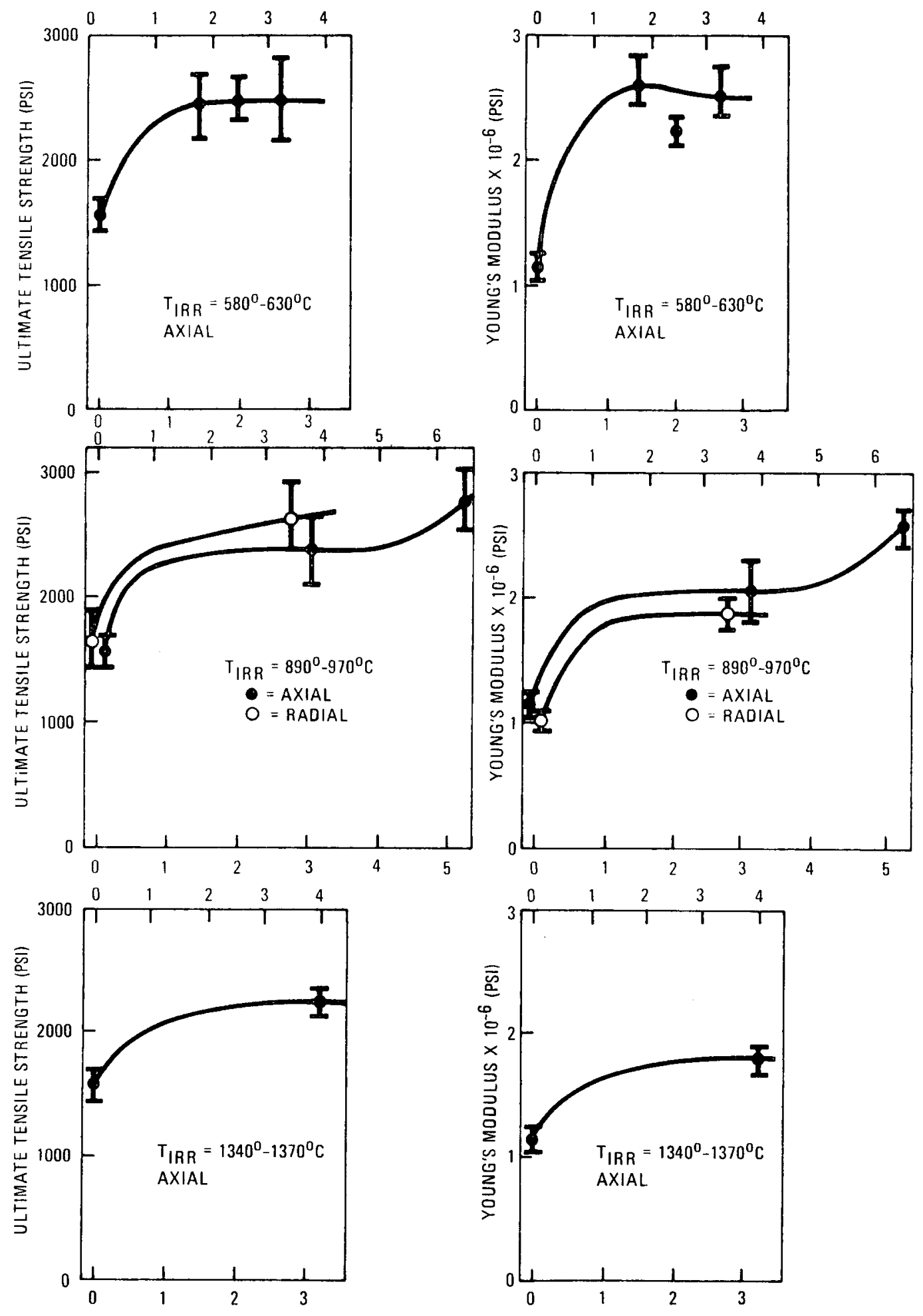

FAST NEUTRON FLUENCE $\times 10^{-21}\left(\mathrm{~N} / \mathrm{CM}^{2}\right)(\mathrm{E}>0.18 \mathrm{MeV}) \mathrm{HTGR}$

Fig. 7-1. Observed tensile strength and Young's modulus of H-451 nearisotropic graphite as functions of fast neutron fluence 

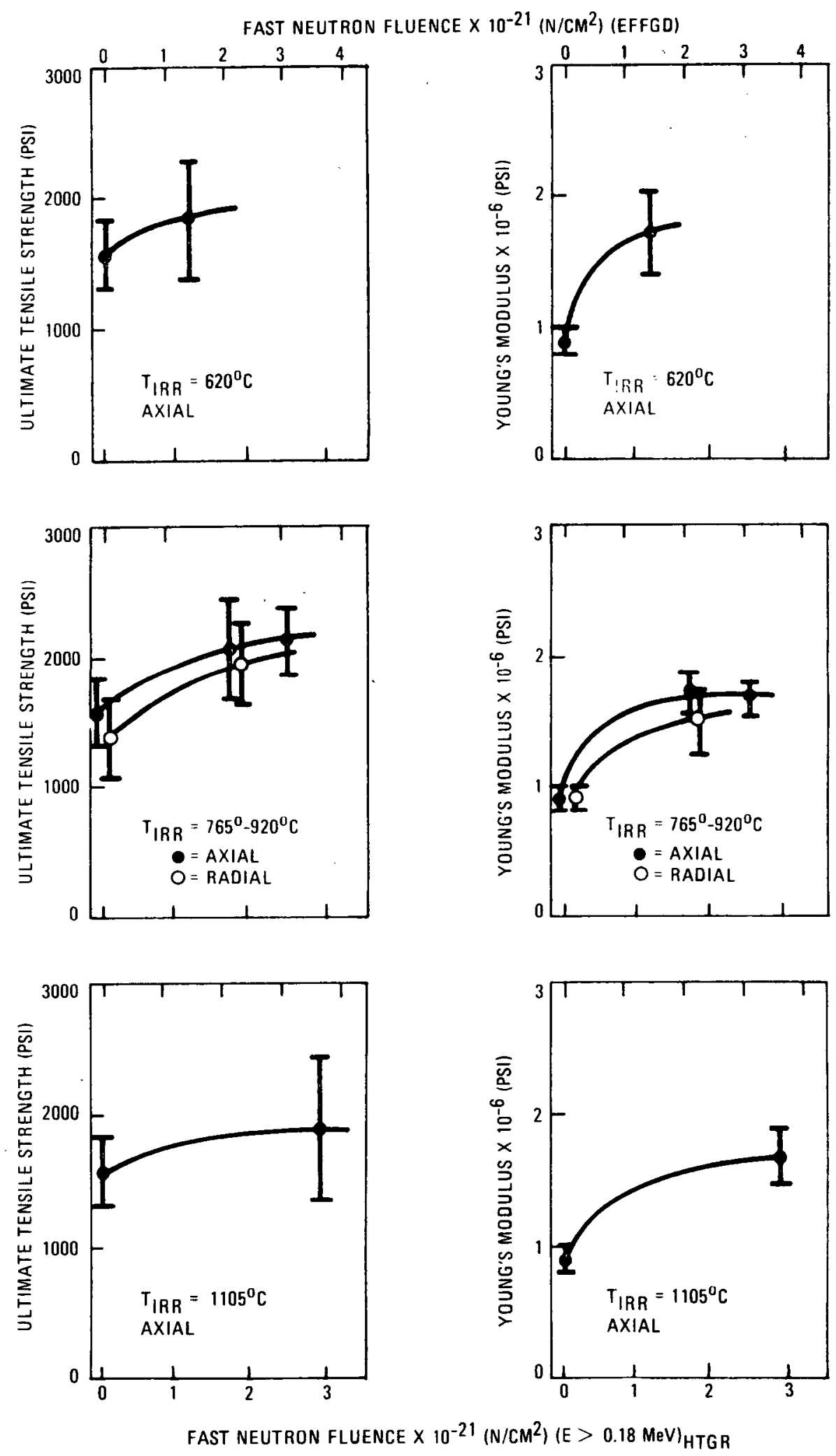

Fig. 7-2. Observed tensile strength and Young's modulus of TS-1240 nearisotropic graphite as functions of fast neutron fluence 

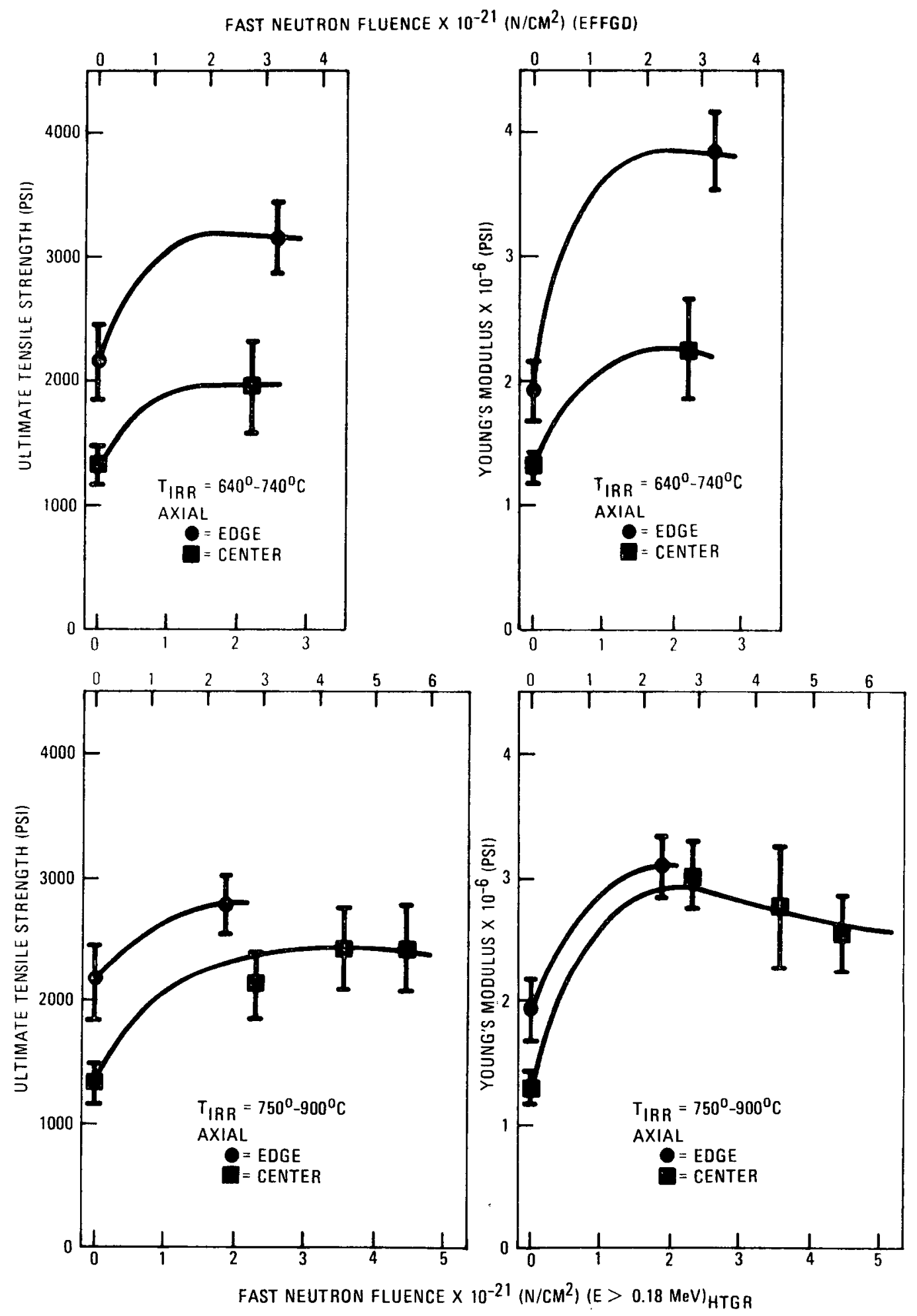

Fig. 7-3(a). Observed tensile strength and Young's modulus of $\mathrm{H}-327$ needle coke graphite as functions of fast neutron fluence at irradiation temperatures of $640^{\circ}$ to $900^{\circ} \mathrm{C}$ 

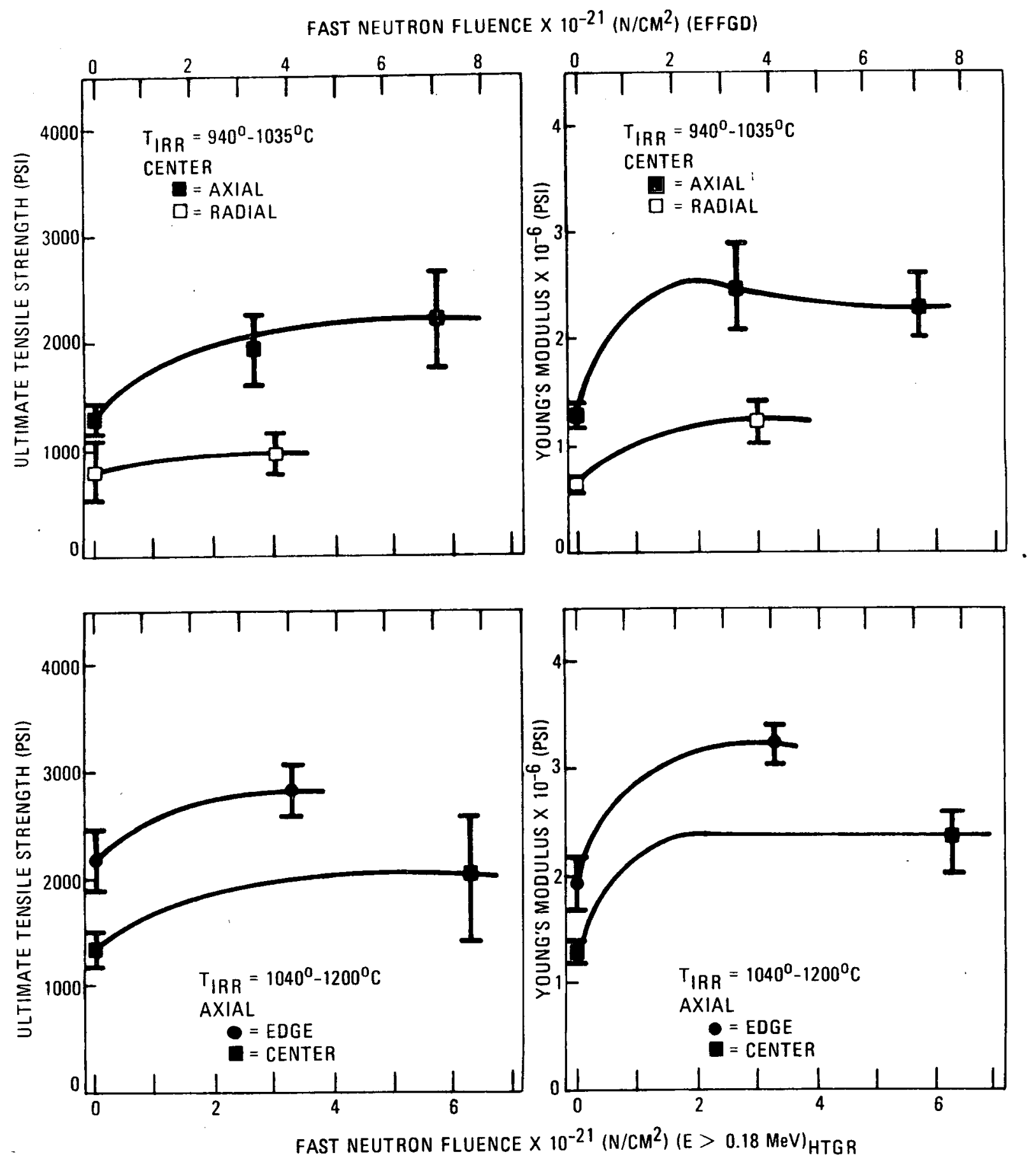

Fig. 7-3(b). Observed tensile strength and Young's modulus of H-327 needle coke graphite as functions of fast neutron fluence at irradiation temperatures of $940^{\circ}$ to $1200^{\circ} \mathrm{C}$ 
TABLE $7-4$

SUMMARY OF MECHANICAL PROPERTY DATA FOR GRAPHITES IRRADIATED IN CAPSULES OG-1 AND OG-2

\begin{tabular}{|c|c|c|c|c|c|c|c|c|}
\hline \multirow[b]{2}{*}{ Material } & \multirow[b]{2}{*}{ Orientation } & \multirow{2}{*}{$\begin{array}{l}\text { Location } \\
\text { in Log }\end{array}$} & \multirow{2}{*}{$\begin{array}{l}\text { Mean } \\
\text { Irradiation } \\
\text { Temperature } \\
\quad\left({ }^{\circ} \mathrm{C}\right)\end{array}$} & \multirow{2}{*}{$\begin{array}{c}\text { Fluence } \times 10^{-21} \\
\left(\mathrm{n} / \mathrm{cm}^{2}\right) \\
(\mathrm{E}>0.18 \mathrm{MeV})_{\mathrm{HTGR}}\end{array}$} & \multicolumn{2}{|c|}{$\begin{array}{l}\text { U1timate Tensile } \\
\text { Strength (psi) }\end{array}$} & \multicolumn{2}{|c|}{ Young's Modulus ( $\left.10^{6} \mathrm{psi}\right)$} \\
\hline & & & & & $\begin{array}{l}\text { Observed } \\
( \pm \text { Std Dev) }\end{array}$ & Predicted $(a)$ & $\begin{array}{l}\text { Observed } \\
( \pm \text { Std Dev) }\end{array}$ & Predicted $^{(a)}$ \\
\hline$\stackrel{H-451}{H}$ & $\mid$ & $\begin{array}{c}\text { Midlength- } \\
\text { center }\end{array}$ & $\begin{array}{c}-- \\
580-630 \\
580-630 \\
580-630 \\
910-950 \\
890-970 \\
1340-1370 \\
-- \\
900-950\end{array}$ & $\begin{array}{c}0 \\
\\
1.4 \\
2.0 \\
2.6 \\
3.0 \\
5.3 \\
3.2 \\
0 \\
2.8\end{array}$ & $\begin{array}{l}1564 \pm 135 \\
2424 \pm 265 \\
2485 \pm 177 \\
2481 \pm 322 \\
2371 \pm 271 \\
2777 \pm 261 \\
2233 \pm 115 \\
1671 \pm 242 \\
2648 \pm 270\end{array}$ & $\begin{array}{c}-- \\
2140 \\
2160 \\
2180 \\
2040 \\
2200 \\
2000 \\
-- \\
2190\end{array}$ & $\begin{array}{l}1.14 \pm 0.09 \\
2.63 \pm 0.20 \\
2.22 \pm 0.11 \\
2.54 \pm 0.20 \\
2.06 \pm 0.24 \\
2.55 \pm 0.15 \\
1.79 \pm 0.08 \\
1.00 \pm 0.05 \\
1.88 \pm 0.13\end{array}$ & $\begin{array}{l}-- \\
2.2 \\
2.2 \\
2.2 \\
2.0 \\
2.4 \\
1.9 \\
-- \\
1.8\end{array}$ \\
\hline $\mathrm{TS}-1240$ & $\left.\right|^{\text {Axial }}$ & $\begin{array}{l}\text { Midlength- } \\
\text { center }\end{array}$ & $\begin{array}{c}-- \\
620 \\
765 \\
920 \\
1105 \\
-- \\
805\end{array}$ & $\begin{array}{c}0 \\
\\
1.2 \\
1.7 \\
2.5 \\
2.9 \\
0 \\
1.7\end{array}$ & $\begin{array}{l}1571 \pm 252 \\
1844 \pm 452 \\
2070 \pm 385 \\
2123 \pm 259 \\
1899 \pm 540 \\
1383 \pm 298 \\
1951 \pm 327\end{array}$ & $\begin{array}{c}-- \\
2140 \\
2100 \\
2070 \\
2050 \\
-- \\
1850\end{array}$ & $\begin{array}{l}0.90 \pm 0.10 \\
1.72 \pm 0.32 \\
1.72 \pm 0.17 \\
1.66 \pm 0.13 \\
1.69 \pm 0.21 \\
0.90 \pm 0.11 \\
1.50 \pm 0.26\end{array}$ & $\begin{array}{l}-- \\
1.7 \\
1.6 \\
1.6 \\
1.6 \\
-- \\
1.6\end{array}$ \\
\hline$\stackrel{\mathrm{H}-327}{.}$ & $\mid$ & $\begin{array}{l}\text { Midlength- } \\
\text { center }\end{array}$ & $\begin{array}{c}-- \\
740 \\
750-810 \\
835-850 \\
860-900 \\
960-1035 \\
940-1035 \\
1040-1200 \\
-- \\
640-700 \\
770-810 \\
1050-1100 \\
-- \\
960-1020\end{array}$ & $\begin{array}{c}0 \\
\\
2.2 \\
3.6 \\
4.5 \\
2.3 \\
2.7 \\
5.7 \\
6.3 \\
0 \\
2.6 \\
1.9 \\
3.3 \\
0 \\
3.0\end{array}$ & $\begin{array}{r}1321 \pm 158 \\
1944 \pm 377 \\
2423 \pm 341 \\
2435 \pm 361 \\
2127 \pm 281 \\
1940 \pm 331 \\
2222 \pm 468 \\
2003 \pm 585 \\
2186 \pm 293 \\
3152 \pm 278 \\
2787 \pm 249 \\
2822 \pm 254 \\
820 \pm 286 \\
962 \pm 198\end{array}$ & $\begin{array}{c}-- \\
1790 \\
1800 \\
1810 \\
1740 \\
1730 \\
1890 \\
1850 \\
-- \\
3000 \\
2930 \\
2860 \\
-- \\
1070\end{array}$ & $\begin{array}{l}1.30 \pm 0.12 \\
2.25 \pm 0.40 \\
2.77 \pm 0.49 \\
2.55 \pm 0.32 \\
3.02 \pm 0.27 \\
2.48 \pm 0.40 \\
2.32 \pm 0.31 \\
2.32 \pm 0.30 \\
1.93 \pm 0.25 \\
3.85 \pm 0.33 \\
3.10 \pm 0.26 \\
3.23 \pm 0.19 \\
0.64 \pm 0.08 \\
1.23 \pm 0.20\end{array}$ & $\begin{array}{l}-- \\
2.4 \\
2.4 \\
2.5 \\
2.3 \\
2.3 \\
2.6 \\
2.5 \\
-- \\
3.7 \\
3.5 \\
3.4 \\
-- \\
1.1\end{array}$ \\
\hline
\end{tabular}

(a) Predicted from Figs. 6-5 and 6-6. 
Young's modulus found during the Dragon project work (Ref. 8). The difference probably occurs because a static determination of Young's modulus on unirradiated graphite is usually lower than a dynamic determination, but the two methods give similar results on irradiated graphite. The present results become consistent with Dragon project data on similar graphites if it is assumed that the static Young's modulus of unirradiated graphite is $80 \%$ of the dynamic modulus, while the two values are the same for irradiated graphite (see Section 8.4 ).

Increases in tensile strength followed a pattern similar to the increases in Young's modulus; but for given irradiation conditions, the fractional increase in strength was always less than the fractional increase in Young's modulus. The condition of constant strain energy to failure would require that the irradiated and unirradiated strengths, $S_{i}$ and $S_{0}$, be related to the irradiated and unirradiated Young's moduli, $E_{i}$ and $E_{0}$, as follows:

$$
\frac{s_{i}}{s_{0}}=\sqrt{\frac{E_{i}}{E_{0}}} .
$$

The strengths of all groups of irradiated $\mathrm{H}-451$ specimens and most groups of H-327 specimens were higher than expected from this relationship, but the strengths of the TS-1240 specimen groups and some H-327 specimen groups were about as predicted.

Irradiation-induced changes in the coefficient of variation of the strength (standard deviation divided by the mean) were not statistically significant, with the exception of the specimens of TS-1240 irradiated at $1105^{\circ} \mathrm{C}$ to $2.9 \times 10^{21} \mathrm{n} / \mathrm{cm}^{2}$ and the $\mathrm{H}-327$ specimens irradiated at $940^{\circ}$ to $1035^{\circ} \mathrm{C}$ to $5.7 \times 10^{21} \mathrm{n} / \mathrm{cm}^{2}$ and at $1040^{\circ}$ to $1200^{\circ} \mathrm{C}$ to $6.3 \times 10^{21} \mathrm{n} / \mathrm{cm}^{2}$. For these specimens there was a significant increase in the coefficient of variation at the $95 \%$ confidence level. 


\section{IMPACT OF TEST DATA ON CURRENT DESIGN PROCEDURES}

\subsection{DIMENSIONAL CHANGES}

The additional data generated by the OG-2 experiment agree very well with design curves fit and extrapolated from earlier data. The only significant change in H-451 design curves is caused by the shift to HTGR fluence. This will result in slightly larger (10\% to $20 \%$ ) dimensional changes at $8 \times 10^{21} \mathrm{n} / \mathrm{cm}^{2}(\mathrm{E}>0.18 \mathrm{MeV})_{\mathrm{HTGR}}$.

\subsection{THERMAL EXPANSIVITY}

Figures 6-1(a) and 6-1(b) show the irradiation-induced fractional changes in thermal expansivity at various irradiation temperatures. Compared with the design curves currently in use, the new data show a somewhat smaller increase at low irradiation temperatures and a greater decrease at high irradiation temperatures. Compared with the current curves, the new data indicate thermal expansivities for near-isotropic graphite that are lower by about $0.5 \times 10^{-6}{ }^{\circ} \mathrm{C}^{-1}$ at fluences of 5 to $7 \times 10^{21} \mathrm{n} / \mathrm{cm}^{2}$ at or above $900^{\circ} \mathrm{C}$.

\subsection{THERMAL CONDUCTIVITY}

New parameters to be used in the thermal conductivity model currently employed (Ref. 7) are 1isted in Table 6-7. For near-isotropic graphites, the new values correspond to thermal conductivities of irradiated graphite about $5 \%$ lower than the current values. For H-327 graphite, the new values are $15 \%$ to $25 \%$ lower than current design values. 


\subsection{MECHANICAL PROPERTIES}

Current design basis curves for the fractional increase in Young's modulus and strength as a function of fluence and temperature were derived from Dragon project sonic modulus data on comparable near-isotropic graphites (Ref. 8), based on the following assumptions:

1. Neutron fluence in units of $\mathrm{n} / \mathrm{cm}^{2}(\mathrm{NDE})=0.6 \times$ fluence in units of $\mathrm{n} / \mathrm{cm}^{2}(\mathrm{E}>0.18 \mathrm{MeV})_{\mathrm{ORR}}\left[0.67 \mathrm{x}\right.$ fluence in units of $\mathrm{n} / \mathrm{cm}^{2}$ $(\mathrm{E}>0.18 \mathrm{MeV})_{\mathrm{HTGR}}$ ].

2. Static Young's modulus of unirradiated graphite $=0.8 \times$ dynamic Young's modulus.

3. Static Young's modulus of irradiated graphite = dynamic Young's modulus.

4. Strength (S) and Young's modulus (E) of unirradiated and irradiated specimens are related as follows:

$$
\frac{S_{i}}{S_{0}}=\sqrt{\frac{E_{i}}{E_{0}}} .
$$

The current curves are reproduced in Figs. 8-1 and 8-2. Modulus and strength values predicted from Figs. $8-1$ and $8-2$ are included in Table 7-4 for comparison with the measurements. The modulus values are in fairly good agreement with the design curve predictions. Observed strengths for $\mathrm{H}-451$ graphite and most of the $\mathrm{H}-327$ specimen groups are higher than predicted. However, there are not yet sufficient experimental measurements to justify the use of a less conservative prediction. No changes are proposed in the strength and Young's modulus design curves (Figs. 8-1 and $8-2)$. 


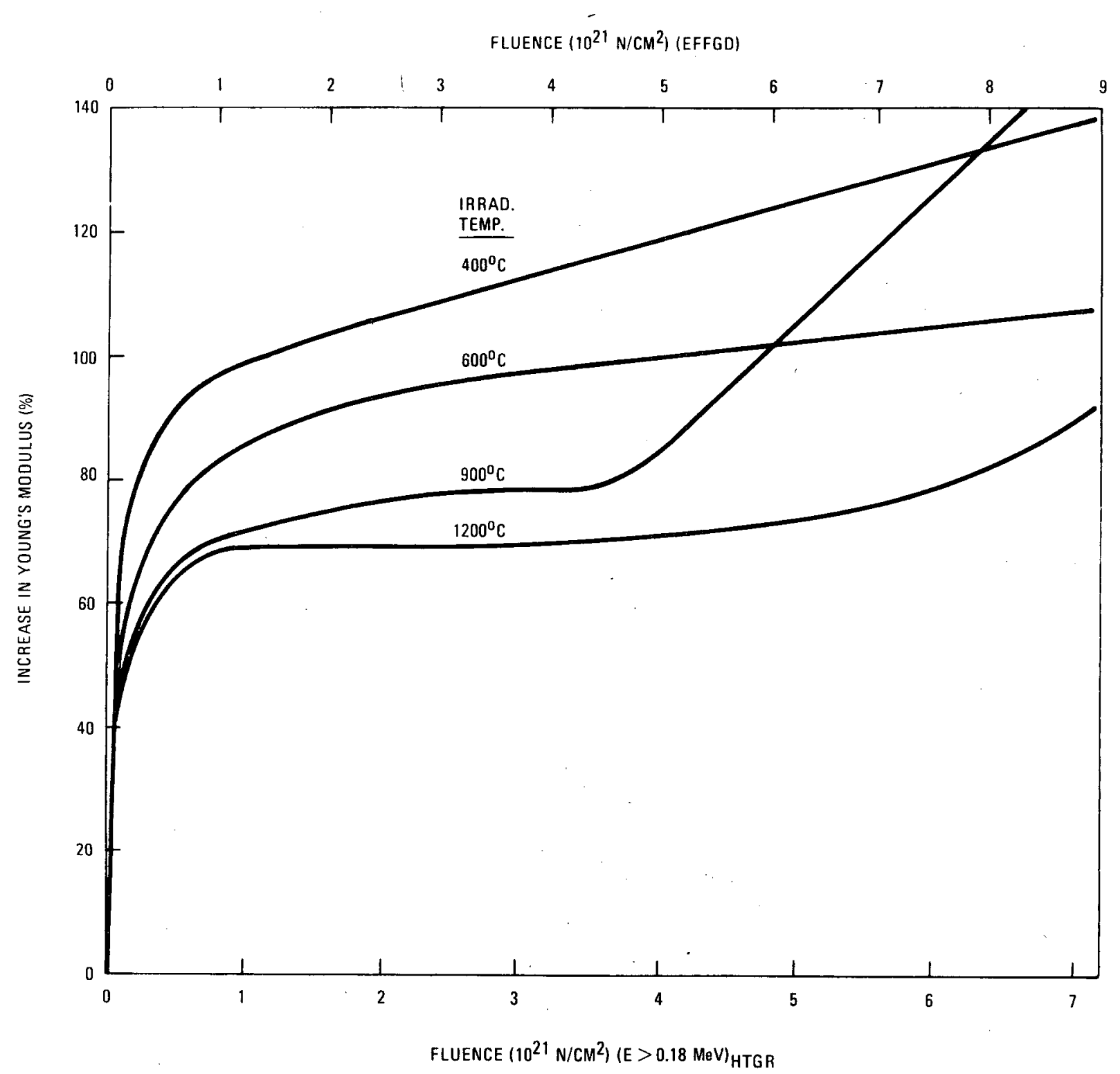

Fig. 8-1. Calculated curves for the percent increase in Young's modulus of near-isotropic graphites as a function of fast neutron fluence and irradiation temperature 


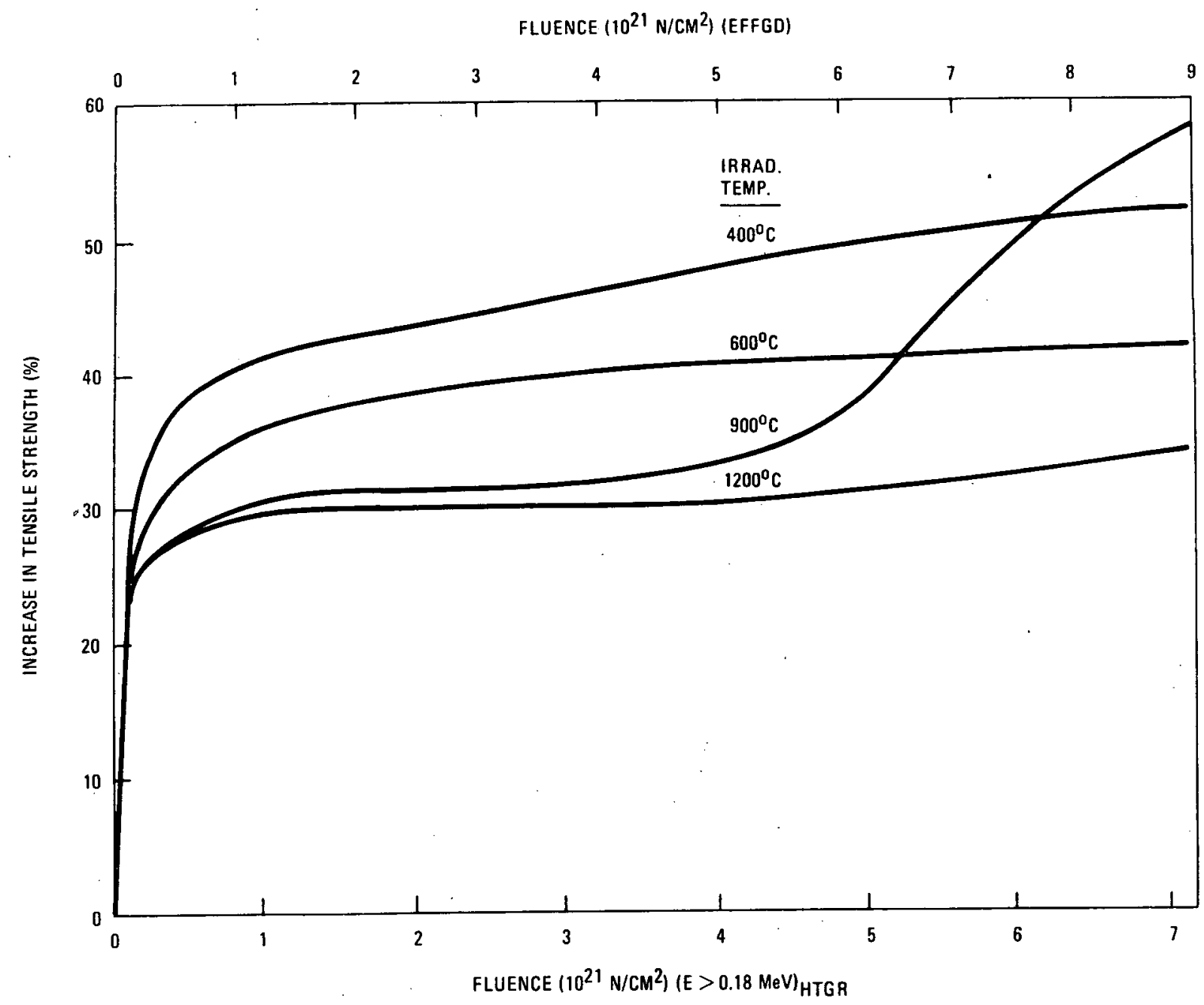

Fig. 8-2. Calculated curves for the percent increase in tensile strength of near-isotropic graphites as a function of fast neutron fluence and irradiation temperature 


\section{REFERENCES}

1. McNair, J. M., "Fabrication and Assembly Report - OG-2," Capsule Irradiation Branch Document No. OG-7, General Atomic Company, October 1974 (unpublished data).

2. Wattier, J. B., "Fina1 Test Operation Report - OG-2," Capsule Irradiation Branch Document No. OG-2-2, General Atomic Company, June 1975 (unpublished data).

3. Beavan, L. A., "Materials Inventory: OG-2 Irradiation Capsule," General Atomic Report GA-D13142, September 1974 (unpublished data).

4. Price, R. J., and L. A. Beavan, "Final Report on Graphite Irradiation Test OG-1," USAEC Report GA-A13089, General Atomic Company, August 1, 1974 .

5. Morgan, W. C., "Neutron Fluence and Atomic Displacement Rates for Graphite Irradiations," Nucl. Technol. 21, 50 (1974).

6. Price, R. J., "Property Changes in Near-Isotropic Graphites Irradiated at $300^{\circ}$ to $600^{\circ} \mathrm{C}$ : A Literature Survey," USAEC Report GA-A13478, General Atomic Company, June 13, 1975.

7. Price, R. J., "Review of the Thermal Conductivity of Nuclear Graphite Under HTGR Conditions," Gulf General Atomic Report Gulf-GA-A12615 (GA-LTR-3), September 7, 1973.

8. Everett, M. R., et al., "Irradiation Performance and Selection of Graphites for HTGRs," Dragon Project Report DP-Report-877, April 1974. 